\title{
Sicherheitsbewertung von Fahrradstraßen und der Öffnung von Einbahnstraßen
}

Norbert Schläger

Benjamin Wührl

Torben Woywod

Andrea Fromberg

Peter Gwiasda

Kirsten Niklas

Marcel Schreiber

Maria Pohle

Unfallforschung der Versicherer 


\section{Sicherheitsbewertung von Fahrradstraßen und der Öffnung von Einbahnstraßen}

Norbert Schläger

Benjamin Wührl Torben Woywod Andrea Fromberg

Peter Gwiasda

Kirsten Niklas

Marcel Schreiber

Maria Pohle 


\section{Impressum}

Gesamtverband der Deutschen Versicherungswirtschaft e. V. Unfallforschung der Versicherer

Wilhelmstraße 43/43G, 10117 Berlin

Postfach 0802 64, 10002 Berlin

E-Mail: unfallforschung@gdv.de

Internet: www.udv.de

Facebook: www.facebook.com/unfallforschung

Twitter: @unfallforschung

YouTube: www.youtube.com/unfallforschung

ISBN-Nr.: 978-3-939163-69-5

Redaktion: Dipl.-Ing. Marcel Schreiber

Erschienen: 09/2016 


\section{Sicherheitsbewertung von Fahrradstraßen und der Öffnung von Einbahnstraßen}

Planungsbüro V I A eG

Marspfortengasse 6

50667 Köln

Fon 0221/789527-28

Fax 0221/789527-99

viakoeln@viakoeln.de

www.viakoeln.de

Andrea Fromberg

Peter Gwiasda

Kirsten Niklas

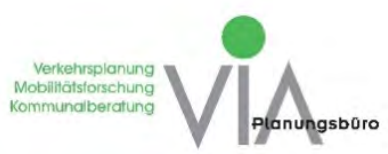

Planerbüro Südstadt: Büro für urbane Mobilität

Antwerpener Str. 6-12

50672 Köln

Fon 0221/20894-0

Fax 0221/20894-44

info@planerbuero-suedstadt.de www.planerbuero-suedstadt.de

Norbert Schläger

Benjamin Wührl

Torben Woywod

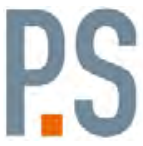

Planerbüro Südstadt:

Büro für urbane Mobilität

Bei der UDV betreut von:

Dipl.-Ing. Marcel Schreiber Dipl.-Ing Maria Pohle

Unfallforschung der Versicherer $7 \mathrm{GDV}$ 


\section{Inhalt}

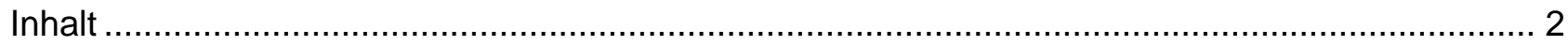

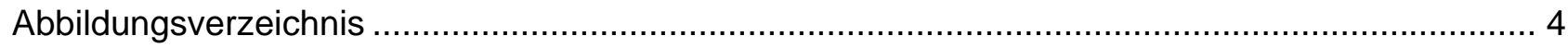

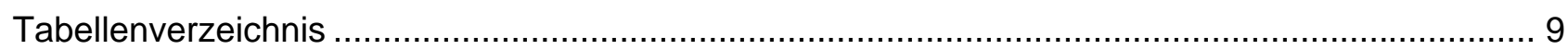

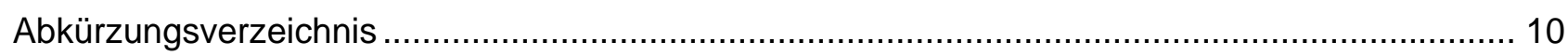

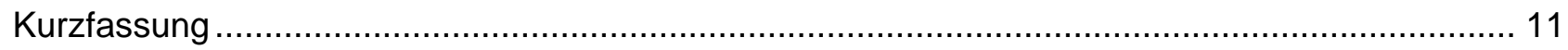

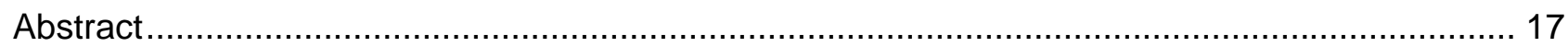

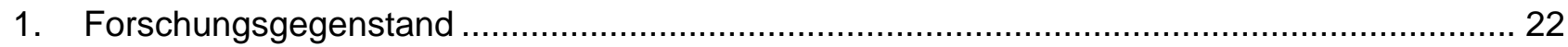

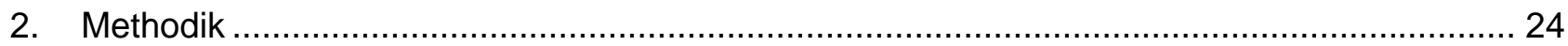

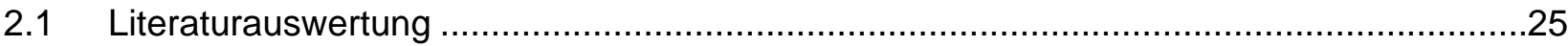

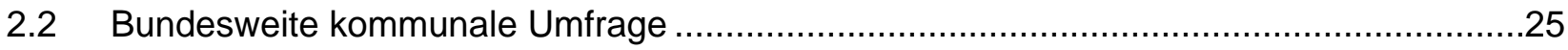

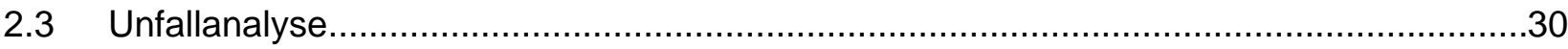

2.3.1 Unfallanalyse in Fahrradstraßen: Datengrundlage und Datenaufbereitung ..........................30

2.3.2 Unfallanalyse in geöffneten Einbahnstraßen: Datengrundlage und Datenaufbereitung.......35

2.4 Analyse und Beobachtung des Verkehrsverhaltens ..................................................40

2.5 Befragung von Verkehrsteilnehmern........................................................................... 43

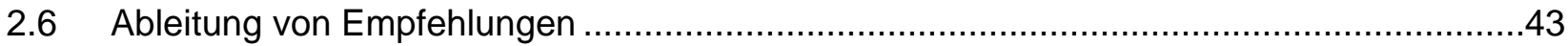

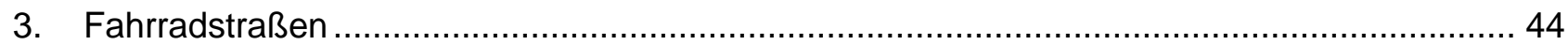

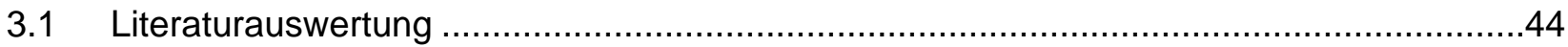

3.1.1 Definitionen, rechtliche Grundlagen und Planungsrichtlinien ...........................................44

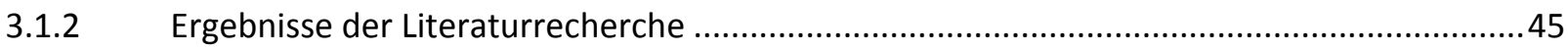

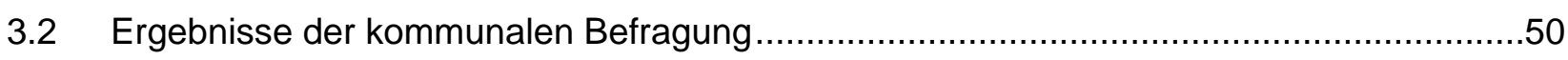

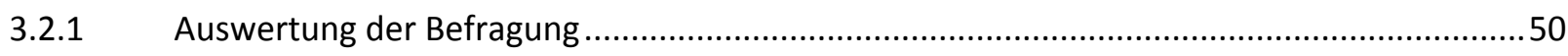

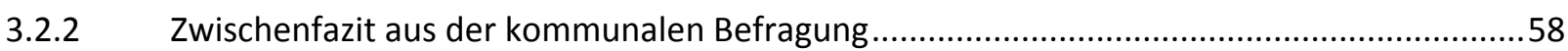

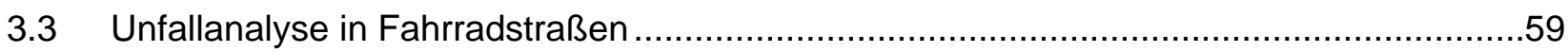

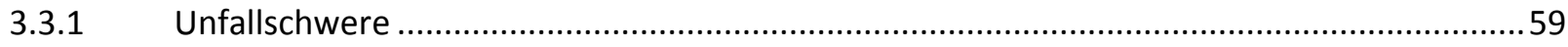

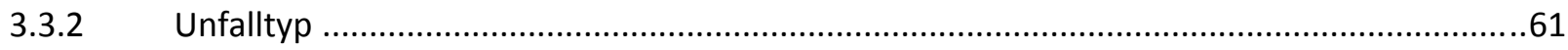

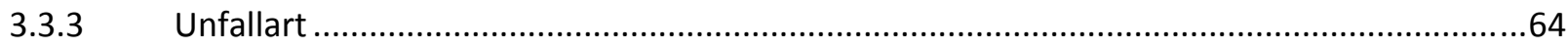

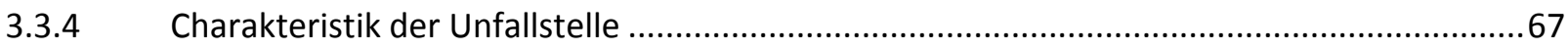

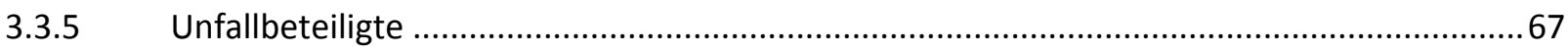

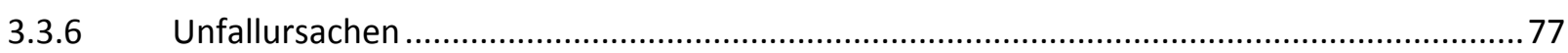

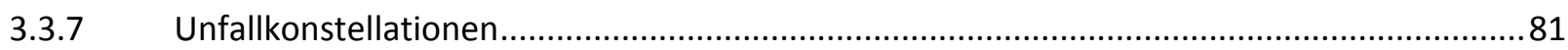

3.3.8 Zwischenfazit zur Unfalluntersuchung in Fahrradstraßen....................................................85

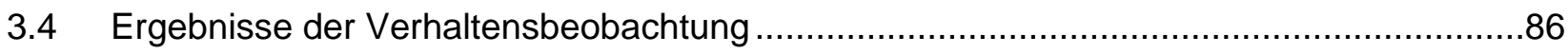

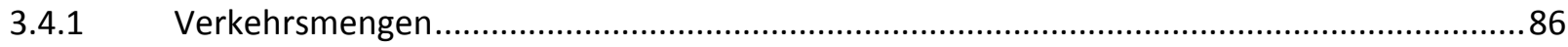




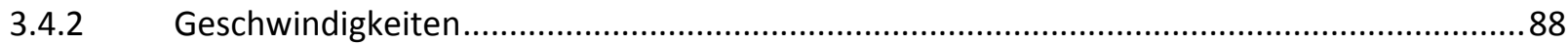

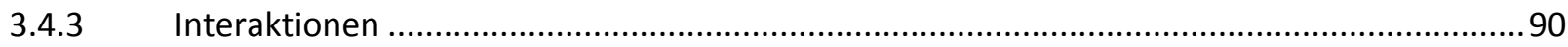

3.4.4 Zwischenfazit der Verhaltensbeobachtung …......................................................................94

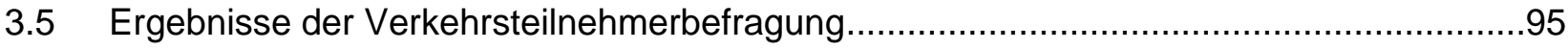

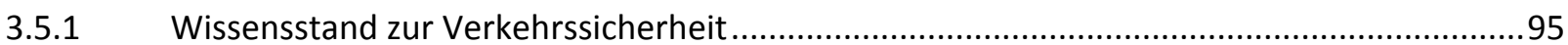

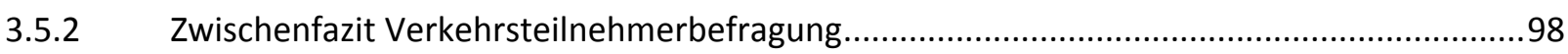

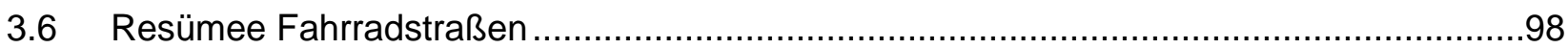

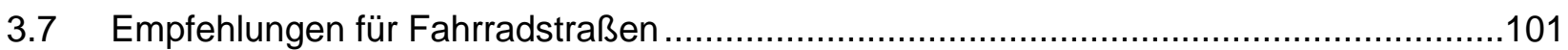

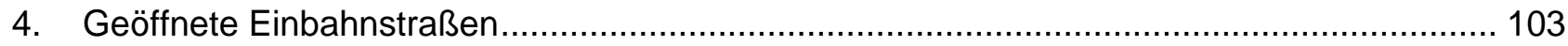

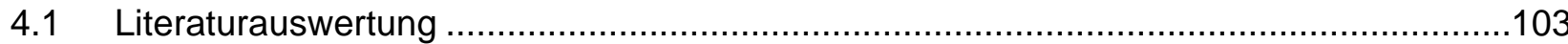

4.1.1 Definitionen, rechtliche Grundlagen und Planungsrichtlinien ...........................................103

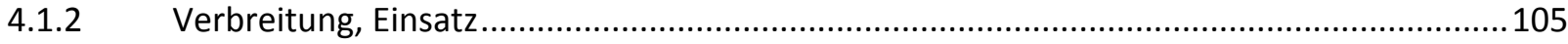

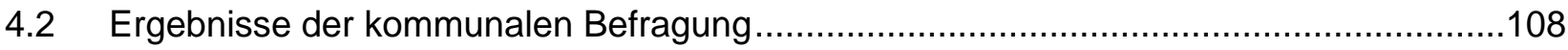

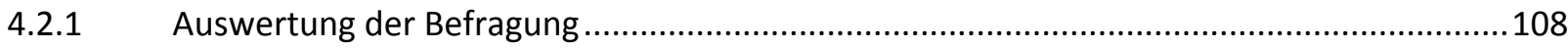

4.2.2 Zwischenfazit aus der kommunalen Befragung..........................................................111

4.3 Unfallanalyse geöffnete Einbahnstraßen ...........................................................111

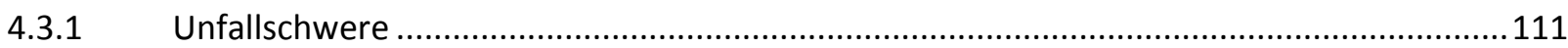

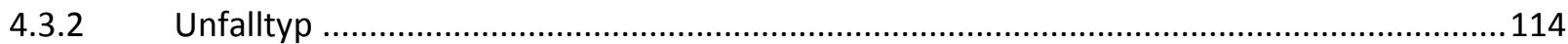

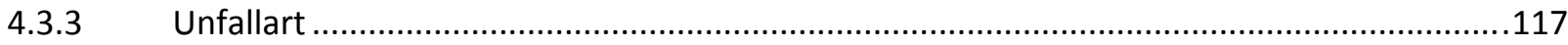

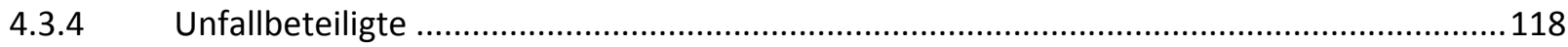

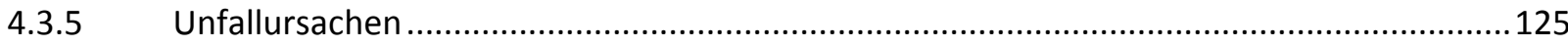

4.3.6 Zwischenfazit Unfalluntersuchung in geöffneten Einbahnstraßen ....................................127

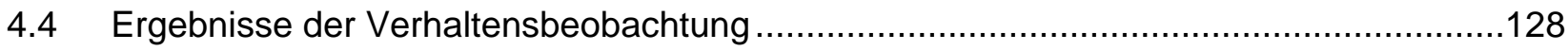

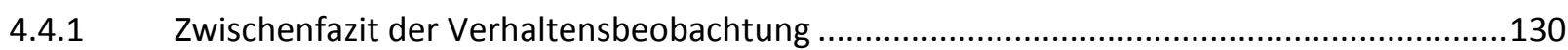

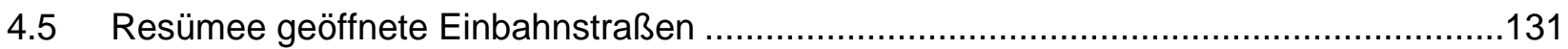

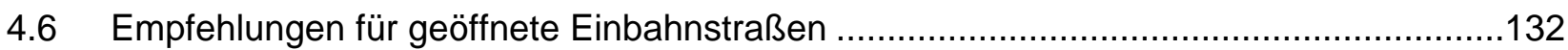

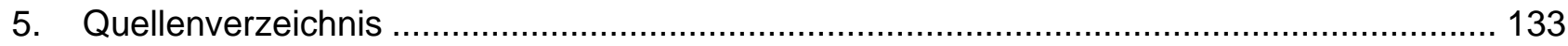

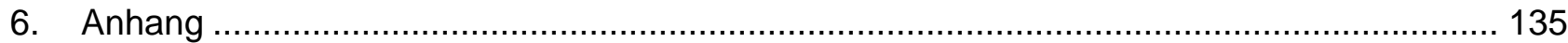




\section{Abbildungsverzeichnis}

Abbildung 1: Ablauf des Forschungsprojektes

Abbildung 2: Verteilung über das Bundesgebiet: Stichprobe $(n=1.076)$ und Teilnehmer an der Befragung ( $n=359)$

Abbildung 3: Verteilung der teilnehmenden Kommunen nach Bundesländern in \% ( $\mathrm{n}=$ 359).

Abbildung 4: Verteilung der teilnehmenden Kommunen auf die Ortsgrößenklassen $(n=359)$

Abbildung 5: Beteiligung an der Kommunalbefragung nach Kommunengröße $(n=1.076) \ldots 28$

Abbildung 6: Verteilung der Stichprobe Phase II - Kommunen mit Fahrradstraßen

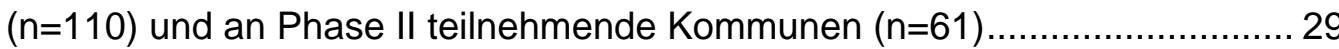

Abbildung 7: Kommunen, die Fahrradstraßen gemeldet haben .....

Abbildung 8: Verhältnis relevanter und nicht relevanter Unfälle in Fahrradstraßen für die mikroskopische Unfalluntersuchung (U(P), 2008-2012).

Abbildung 9: Unfallort der relevanten Radverkehrsunfälle in Fahrradstraßen (Unfälle mit Personenschaden (U(P), 2008-2012) $(n=186)$.

Abbildung 10:Kommunen, die problematische, geöffnete Einbahnstraßen gemeldet haben 36

Abbildung 11:Verhältnis relevanter und nicht relevanter Unfälle in geöffneten

Einbahnstraßen in der bundesweiten Untersuchung für die mikroskopische

Unfalluntersuchung (Unfälle mit Personenschaden (U(P), 2008-2012).....

Abbildung 12:Verhältnis relevanter und nicht relevanter Unfälle in geöffneten

Einbahnstraßen in der Sonderuntersuchung Kölner Innenstadt für die mikroskopische Unfalluntersuchung (Unfälle mit Personenschaden $(U(P)$, 2008-2012)

Abbildung 13:Unfallort der relevanten Radverkehrsunfälle in geöffneten Einbahnstraßen bundesweit und in der Sonderuntersuchung in der Kölner Innenstadt

(Unfälle mit Personenschaden (U(P), 2008-2012) ( $n=54$ bzw. $n=63) \ldots \ldots \ldots \ldots . . . .40$

Abbildung 14: Systematik der Begegnungsfälle

Abbildung 15:Beginn einer Fahrradstraße, Zeichen 244.1 StVO ....................................... 44

Abbildung 16: Ende einer Fahrradstraße, Zeichen 244.2 StVO .......................................... 44

Abbildung 17: Beispiele für die Zulassung anderer Verkehrsarten in Fahrradstraßen .......... 45

Abbildung 18: Beispiele für Flyer zum Thema „Fahrradstraßen“ (ADFC BRAUNSCHWEIG/STADT BRAUNSCHWEIG) ….................................... 50

Abbildung 19: Einsatz von Fahrradstraßen nach Kommunengröße ( $\mathrm{n}=359$ Kommunen) ...... 50

Abbildung 20: Geplante Einrichtung von Fahrradstraßen in den befragten Kommunen in \%

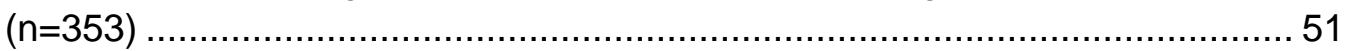

Abbildung 21: Gründe gegen die Ausweisung von Fahrradstraßen ( $\mathrm{n}=130$ Kommunen) ...... 52

Abbildung 22: Einschätzung der Verkehrssicherheit auf den einzelnen Fahrradstraßen

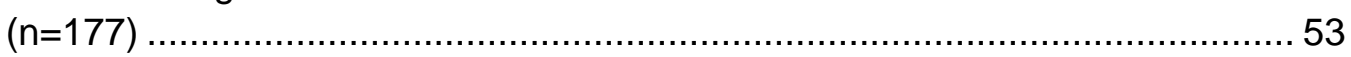

Abbildung 23:Beschwerden aus der Bevölkerung über die einzelnen Fahrradstraßen

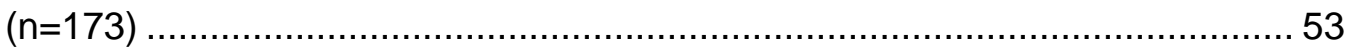

Abbildung 24:Umfang der Maßnahmen für die Gestaltung von Fahrradstraßen $(n=178)$,

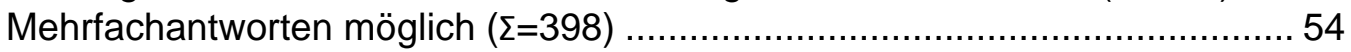

Abbildung 25:Vorfahrtregelung an den erhobenen Fahrradstraßen $(n=178) \ldots \ldots \ldots \ldots \ldots \ldots \ldots . . . . . . . . . .55$ Abbildung 26: Netzbedeutung der Fahrradstraße $(n=177)$, Mehrfachnennungen möglich

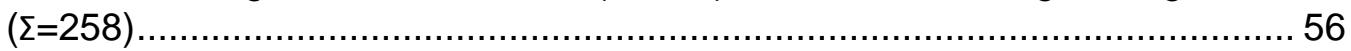

Abbildung 27: Funktion der Fahrradstraße ( $n=177)$, Mehrfachnennungen möglich $(\Sigma=469)$.. 56 Abbildung 28:Zufahrtserlaubnis für andere Verkehrsarten in Fahrradstraßen $(n=179) \ldots \ldots . . .57$ 
Abbildung 29:Parkregelung in den Fahrradstraßen, $n=178$, Mehrfachnennungen wegen

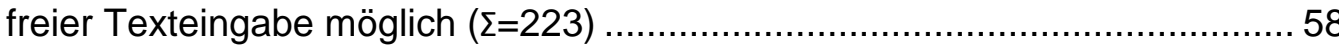

Abbildung 30:Vergleich der Unfallkategorie in Fahrradstraßen mit den bundesweiten Innerortsunfällen (U(P), 2008-2012)

Abbildung 31:Vergleich der Unfallschwere für Radverkehrsunfälle in Fahrradstraßen (Makroanalyse) mit den bundesweiten Innerortsunfällen und den Unfällen in Nebenstraßen (U(P), 2008-2012)

Abbildung 32:Vergleich der Unfallschwere für relevante Radverkehrsunfälle in Fahrradstraßen (Mikroanalyse) mit allen Radverkehrsunfällen in Fahrradstraßen (Makroanalyse) und den bundesweiten Radverkehrsunfällen auf Innerortsstraßen (U(P), 2008-2012)

Abbildung 33:Vergleich des Unfalltyps in Fahrradstraßen mit den bundesweiten Innerortsunfällen (U(P), 2008-2012)

Abbildung 34:Vergleich des Unfalltyps von Radverkehrsunfällen in Fahrradstraßen mit den bundesweiten Innerortsunfällen und mit Nebenstraßen (U(P), 20082012).

Abbildung 35: Vergleich des Unfalltyps für relevante Radverkehrsunfälle (Mikroanalyse) in Fahrradstraßen mit allen Radverkehrsunfällen (Makroanalyse) in Fahrradstraßen und den bundesweiten Radverkehrsunfällen auf Innerortsstraßen (U(P), 2008-2012)

Abbildung 36: Vergleich des Unfalltyps für relevante Radverkehrsunfälle in Fahrradstraßen an Zwischenknotenpunkten mit denen auf der Strecke (U(P), 2008-2012) .... 64

Abbildung 37:Vergleich der Unfallart in Fahrradstraßen mit den bundesweiten Innerortsunfällen (U(P), 2008-2012)

Abbildung 38:Vergleich der Unfallart in Fahrradstraßen mit und ohne

Radverkehrsbeteiligung, sowie mit den bundesweiten Innerortsunfällen ohne Radverkehrsbeteiligung (U(P), 2008-2012)

Abbildung 39:Vergleich der Charakteristik der Unfallstelle in Fahrradstraßen mit den bundesweiten Innerortsunfällen mit Radfahrerbeteiligung (U(P), 2008-2012.. 67

Abbildung 40:Anteile der Unfälle mit Radverkehrsbeteiligung in Fahrradstraßen in den Analyseschritten (U(P), 2008-2012)

Abbildung 41: Vergleich der Hauptverursacher in Fahrradstraßen (Makroanalyse) mit denen bei bundesweiten Innerortsunfällen (U(P), 2008-2012)

Abbildung 42:Vergleich der Hauptverursacher bei relevanten Radverkehrsunfällen in Fahrradstraßen (Mikroanalyse) mit denen bei allen Radverkehrsunfällen in Fahrradstraßen (Makroanalyse) und den bundesweiten Radverkehrsunfällen auf Innerortsstraßen (U(P), 2008-2012)

Abbildung 43:Vergleich der Hauptverursacher für relevante Radverkehrsunfälle in Fahrradstraßen (Mikroanalyse) an Zwischenknotenpunkten mit denen auf der Strecke (U(P), 2008-2012)

Abbildung 44:Unfallbeteiligte 1 und 2 bei allen Unfällen in Fahrradstraßen in der Makroanalyse (U(P), 2008-2012 nur Beteilige 1 und 2, $n=863$ )

Abbildung 45: Unfallgegner bei Unfällen mit Radverkehrsbeteiligung in Fahrradstraßen in der Makroanalyse $(U(P), 2008-2012 ; n=504$; ohne Alleinunfälle Rad)

Abbildung 46:Unfallbeteiligte 1 und 2 bei Unfällen mit Radverkehrsbeteiligung in

Fahrradstraßen in der Makroanalyse (U(P), 2008-2012, $n=579)$

Abbildung 47: Unfallbeteiligte 1 und 2 bei relevanten Unfällen mit Radverkehrsbeteiligung in Fahrradstraßen in der Mikroanalyse (U(P), 2008-2012, $n=186)$ 
Abbildung 48: Unfallbeteiligte 1 und 2 bei Unfällen mit Radverkehrsbeteiligung in Fahrradstraßen auf der Strecke in der Mikroanalyse (U(P), 2008-2012, $\mathrm{n}=63$ )

Abbildung 49:Unfallbeteiligte 1 und 2 bei Unfällen mit Radverkehrsbeteiligung in Fahrradstraßen im Zwischenknotenpunkt in der Mikroanalyse $(U(P), 2008$ 2012, $\mathrm{n}=65$ )

Abbildung 50: Unfallverursacher bei Unfällen mit Radverkehrsbeteiligung in Fahrradstraßen in der Makroanalyse (U(P), 2008-2012, ohne Alleinunfälle Fahrrad: $n=500$ )

Abbildung 51:Unfallursachen der Unfallgegner von Radfahrern (alle außer Radfahrer) bei Unfällen mit Radverkehrsbeteiligung in Fahrradstraßen in der Makroanalyse $(\mathrm{U}(\mathrm{P}), 2008-2012, \mathrm{n}=379$ Ursachen, Mehrfachnennungen möglich).

Abbildung 52: Unfallursachen der Unfallgegner von Radfahrern (alle außer Radfahrer) bei relevanten Unfällen mit Radverkehrsbeteiligung in Fahrradstraßen in der Mikroanalyse ( $(P), 2008-2012, n=129$ Ursachen, Mehrfachnennungen möglich)

Abbildung 53: Unfallursachen aller beteiligten Radfahrer bei Unfällen in Fahrradstraßen in der Makroanalyse (U(P), 2008-2012, n=421 Ursachen, Mehrfachnennungen möglich)

Abbildung 54:Unfallursachen aller beteiligten Radfahrer bei relevanten Unfällen in

Fahrradstraßen in der Mikroanalyse (U(P), 2008-2012, n=100 Ursachen,

Mehrfachnennungen möglich)....

Abbildung 55:24/h-Werte für Kfz- und Radverkehrsstärken in Fahrradstraßen, n=21

Fahrradstraßen

Abbildung 56:Verkehrsanteil Kfz/Radfahrer in den untersuchten Fahrradstraßen, $\mathrm{n}=21$

Fahrradstraßen.

Abbildung 57:Konfliktraten und Verkehrsstärken für Rad/24h und Kfz/24h.

Abbildung 58: Gemessene Geschwindigkeiten auf den Streckenabschnitten in den

Fahrradstraßen ( $n=1.238 \mathrm{Kfz}$ in 4 Fahrradstraßen).

Abbildung 59: Gemessene Geschwindigkeiten in den schmalen Fahrradstraßen mit hohem Radverkehrsanteil ( $n=507 \mathrm{Kfz}$ in 3 Fahrradstraßen).

Abbildung 60: Gemessene Geschwindigkeiten in der breiten Fahrradstraße mit geringerem Radverkehrsanteil ( $\mathrm{n}=737 \mathrm{Kfz}$ in einer Fahrradstraße).

Abbildung 61:Konflikte nach Fehlerarten (Strecke und Knotenpunkte), $n=80$ Konflikte ........ 91

Abbildung 62:Wartepflichtiges Kfz kommt durch verspätetes Bremsen im Fahrbereich des Radfahrers zum Stehen, Radfahrer weicht aus.

Abbildung 63:Wartepflichtiger Lkw fährt in Kreuzung ein, obwohl Radfahrer sich nähert, abrupte Bremsung des Lkw verhindert Kollision.

Abbildung 64:Wartepflichtiger Pkw quert Fahrradstraße und nimmt Radfahrer die Vorfahrt, dieser reduziert seine Geschwindigkeit.

Abbildung 65:Wartepflichtiger Radfahrer auf der Fahrradstraße nimmt Pkw die Vorfahrt, dieser bremst leicht.

Abbildung 66:Kfz biegt in zu großem Radius in die Fahrradstraße ein, entgegenkommender Radfahrer weicht aus.

Abbildung 67: Kfz überholt Radfahrer, entgegenkommender Radfahrer weicht aus und wird langsamer.

Abbildung 68: Gruppe nebeneinander fahrender Radfahrer macht entgegenkommenden

Kfz nur verzögert Platz, Kfz bremst ab.

Abbildung 69:Fußgänger überschreiten unachtsam die Fahrbahn, Radfahrer weicht aus.... 94 
Abbildung 70:Tür von parkendem Kfz öffnet sich, Radfahrer weicht aus (im hinteren Bildbereich).

Abbildung 71: Verkehrsmittelnutzung der befragten Personen $(n=452)$

Abbildung 72: Wissensstand zu Fahrradstraßen ( $n=452$ Autofahrer, Radfahrer und Fußgänger)

Abbildung 73:Wissensstand zu Fahrradstraßen, Vergleich zwischen Autofahrern- $(n=120)$

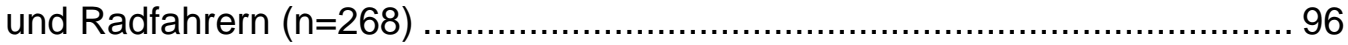

Abbildung 74: Sicherheitsempfinden der Verkehrsteilnehmer nach Art der Verkehrsteilnahme $(n=451)$

Abbildung 75: Subjektives Sicherheitsgefühl: Anteil „unsicher“ je Aspekt, Vergleich zwischen Radfahrer und sonstigen Verkehrsteilnehmern, $\mathrm{n}=452$.

Abbildung 76: Beschilderung nach StVO für die Öffnung von Einbahnstraßen für Fahrradgegenverkehr.

Abbildung 77:Zeichen 267 StVO mit Zusatzzeichen 1022-10.

Abbildung 78: Mögliche StVO-Beschilderungen an Rechts-vor-links-Kreuzungen. 104

Abbildung 79:Räumliche Verteilung der Kommunen mit /ohne geöffnete Einbahnstraßen $(n=260 / n=99)$

Abbildung 80:Kommunen mit geöffneten Einbahnstraßen nach Kommunengröße $(n=311) 110$

Abbildung 81:Vergleich der Unfallkategorie in geöffneten Einbahnstraßen in den einzelnen Analyseschritten mit den bundesweiten Innerortsunfällen $(U(P)$, 2008-2012)

Abbildung 82:Vergleich der Unfallkategorie in geöffneten Einbahnstraßen in Zwischenknotenpunkten und auf der Strecke in der Mikroanalyse und in der Sonderuntersuchung

Abbildung 83:Vergleich des Unfalltyps in geöffneten Einbahnstraßen in den

Analysephasen mit den bundesweiten Innerortsunfällen (U(P), 2008-2012) 114

Abbildung 84: Vergleich des Unfalltyps in geöffneten Einbahnstraßen in

Zwischenknotenpunkten und auf der Strecke.

Abbildung 85:Verortung der relevanten Unfälle mit Radverkehrsbeteiligung in geöffneten Einbahnstraßen in der Kölner Innenstadt nach Unfallkategorie und Unfalltyp116

Abbildung 86:Vergleich der Unfallart in geöffneten Einbahnstraßen mit den bundesweiten Innerortsunfällen (U(P), 2008-2012)

Abbildung 87:Vergleich der ersten Unfallbeteiligten (Hauptverursacher) in geöffneten Einbahnstraßen mit denen bei bundesweiten Innerortsunfällen $(U(P), 2008$ 2012.

Abbildung 88:Vergleich der ersten Unfallbeteiligten (Hauptverursacher) für relevante Unfälle in geöffneten Einbahnstraßen an Zwischenknotenpunkten und auf der Strecke

Abbildung 89:Unfallbeteiligte 1 und 2 bei allen Unfällen in geöffneten Einbahnstraßen $(U(P), 2008-2012$ nur Beteiligte 1 und 2, $n=161)$....

Abbildung 90:Unfallgegner bei Unfällen mit Radverkehrsbeteiligung in geöffneten Einbahnstraßen $(U(P), 2008-2012, n=91$; ohne Alleinunfälle Rad)

Abbildung 91:Erste Unfallbeteiligte (Hauptverursacher) bei Unfällen mit Radverkehrsbeteiligung in geöffneten Einbahnstraßen (U(P), 2008-2012, nur Beteiligte 1 und 2, n=99)

Abbildung 92: Erste Unfallbeteiligte (Hauptverursacher) bei Unfällen der Sonderuntersuchung geöffnete Einbahnstraßen in der Kölner Innenstadt $(U(P), 2008-2012$, nur Beteiligte 1 und 2, n=63)....

Abbildung 93:Unfallverursacher bei Unfällen mit Radverkehrsbeteiligung in geöffneten Einbahnstraßen $(U(P), 2008-2012$, ohne Alleinunfälle Fahrrad: $n=99)$ 
Abbildung 94:Unfallursachen aller beteiligten Radfahrer bei Unfällen mit Radverkehrsbeteiligung in geöffneten Einbahnstraßen in der Makroanalyse $(U(P), 2008-2012, n=57$, Mehrfachnennungen möglich)

Abbildung 95:Unfallursachen aller beteiligten Unfallgegner außer Radfahrer an Unfällen mit Radverkehrsbeteiligung in geöffneten Einbahnstraßen in der Makroanalyse $(U(P), 2008-2012, n=63$, Mehrfachnennungen möglich)

Abbildung 96:Wartepflichtiger Kfz nimmt beim Linkseinbiegen einem Radfahrer in Gegenrichtung der Einbahnstraße die Vorfahrt, dieser bremst deutlich ab... 129

Abbildung 97:Wartepflichtiger Kfz nimmt einem aus Gegenrichtung der Einbahnstraße kommenden Radfahrer die Vorfahrt, dieser muss stark bremsen. 129

Abbildung 98: Kfz biegt in großem Radius in eine Straße ab, entgegenkommender Radfahrer in Gegenrichtung der Eibahnstraße weicht aus. 129

Abbildung 99: Gemessene Geschwindigkeit, Standort $1(n=146)$ 


\section{Tabellenverzeichnis}

Tabelle 1: $\quad$ Übersicht der untersuchten Fahrradstraßen und Unfälle in der Mikroanalyse 32

Tabelle 2: Übersicht der untersuchten geöffneten Einbahnstraßen und Unfälle in der

Makro- und Mikroanalyse ....

Tabelle 3: $\quad$ Übersicht der untersuchten geöffneten Einbahnstraßen und

Radverkehrsunfälle in der Sonderuntersuchung für die Kölner Innenstadt (Radverkehrsunfälle mit Personenschaden (U(P), 2008-2012)

Tabelle 4: $\quad$ Parkregelung bei Streckenunfällen mit Radverkehrsbeteiligung in der Mikroanalyse (Unfälle mit Personenschaden (U(P), 2008-2012)

Tabelle 5: Häufigste Unfallkonstellationen in Zwischenknotenpunkten in der Mikroanalyse (U(P), 2008-2012, $n=65)$.

Tabelle 6: Häufigste Unfallkonstellationen auf der Strecke in der Mikroanalyse $(U(P)$, 2008-2012, n=63) 


\section{Abkürzungsverzeichnis}

Abb. Abbildung

ADFC Allgemeiner Deutscher Fahrradclub

Bast Bundesanstalt für Straßenwesen

BBSR Bundesinstituts für Bau-, Stadt- und Raumforschung

DESTATIS Statistisches Bundesamt

ERA 2010 Empfehlungen für Radverkehrsanlagen, Ausgabe 2010

EUSKa Elektronische Unfalltypen-Steckkarte

Ew. Einwohner

FGSV Forschungsgesellschaft für Straßen- und Verkehrswesen e.V.

Fz. Fahrzeuge

HBS Handbuch für die Bemessung von Straßenverkehrsanlagen

Kap. Kapitel

Kat 1-3 Unfallkategorien 1 bis 3

$\mathrm{Kfz} \quad$ Kraftfahrzeug

Lkw Lastkraftwagen

NRW Nordrhein-Westfalen

Pkw Personenkraftwagen

RASt 06 Richtlinie für die Anlage von Stadtstraßen, Ausgabe 2006

StVO Straßenverkehrs-Ordnung

Tab. Tabelle

$\mathrm{U}(\mathrm{P}) \quad$ Unfall mit Personenschaden

VwV-StVO Allgemeine Verwaltungsvorschrift zur Straßenverkehrs-Ordnung 


\section{Kurzfassung}

Mit diesem Forschungsvorhaben wurden zwei Infrastrukturelemente untersucht, die mittlerweile zu den Standards der Radverkehrsförderung gehören: Fahrradstraßen und für den Radverkehr in Gegenrichtung geöffnete Einbahnstraßen. Die Öffnung von Einbahnstraßen für den Radverkehr in Gegenrichtung, im Weiteren als „geöffnete Einbahnstraßen“ bezeichnet, ist seit der StVO-Novelle 1997 offiziell eingeführt, es existieren ausführlich dokumentierte Untersuchungen zur Verkehrssicherheit und zur Akzeptanz. Fahrradstraßen wurden ebenfalls 1997 eingeführt, wurden aber lange Zeit nicht in dem Maß umgesetzt wie die geöffneten Einbahnstraßen. Erst in den letzten Jahren ist in der kommunalen Verkehrsplanung ein zunehmender Einsatz von Fahrradstraßen festzustellen. Allerdings existieren bisher nur wenige Untersuchungen zur Verkehrssicherheit und zur Akzeptanz von Fahrradstraßen.

Der Einsatzzweck der beiden Elemente unterscheidet sich wesentlich. Mit der Öffnung von Einbahnstraßen wird das Straßennetz für den Radverkehr insgesamt durchlässiger. Radfahrende erhalten dadurch neue umwegfreie Fahrbeziehungen, die Fahrtzeit verkürzt sich. Fahrradstraßen hingegen sollen den Radverkehr bündeln und deshalb vorrangig auf wichtigen Netzbestandteilen des Radverkehrs bei bereits hohen Radverkehrsanteilen eingesetzt werden.

Die Untersuchung hat aktuelle Daten zu Verbreitung, Einsatz, Wahrnehmung und zur Verkehrssicherheit der beiden Infrastrukturelemente erhoben. Für Fahrradstraßen sollten grundlegende Aussagen zur Verkehrssicherheit getroffen werden, um bei der sich andeutenden verstärkten Nutzung in der Praxis zu einem verkehrssicheren Einsatz beizutragen. Für geöffnete Einbahnstraßen wurden Hinweise erwartet, ob die vorliegenden Erkenntnisse zur Verkehrssicherheit weiterhin Bestand haben.

Die Untersuchungen zu beiden Instrumenten wurden weitgehend getrennt durchgeführt, wobei die Untersuchungsmethodik dem gleichen Schema folgte. Nach einer Literaturanalyse wurde eine bundesweite kommunale Onlinebefragung durchgeführt, an der 359 Kommunen teilgenommen haben, um die Verbreitung der beiden Elemente festzustellen. Dabei wurden auch Praxisbeispiele erfragt, um für die weitere Bearbeitung konkrete Untersuchungsfälle zu erhalten. Für 181 Fahrradstraßen und 18 geöffnete Einbahnstraßen wurden im Folgenden die Unfalldaten aus fünf Kalenderjahren (2008 - 2012) nach makroskopischen Gesichtspunkten untersucht. Zu den geöffneten Einbahnstraßen wurden nur wenige unfallauffällige Straßen gemeldet, so dass wegen der kleinen Stichprobe alle geöffneten Einbahnstraßen in der Kölner Innenstadt als zusätzliches Untersuchungskollektiv in die Analyse mit einbezogen worden. Da aus der makroskopischen Umfallanalyse keine signifikanten Unterschiede zu den Nebenstraßen heraus gearbeitet werden konnten, wurde eine vertiefende mikroskopische Unfallanalyse an 177 Fahrradstraßen und 31 Einbahnstraßen durchgeführt, d.h. auch die Unfallhergänge wurden analysiert.

Aus den Ergebnissen der Kommunalbefragung und der Unfallanalyse wurden 26 Standorte für eine Videobeobachtung ausgewählt, davon 21 in Fahrradstraßen und fünf in geöffneten Einbahnstraßen. An 21 Knotenpunkten und fünf Streckenabschnitten wurden Verkehrsdaten (Verkehrsmengen und Interaktionen) ermittelt und an den Strecken zusätzlich die Geschwindigkeiten erhoben. Für die Fahrradstraßen wurde ergänzend eine Verkehrsteilnehmerbefragung durchgeführt, um die Regelkenntnis und das subjektive Sicherheitsgefühl von Kraftfahrern und Radfahrern in diesen Straßen zu ermitteln. 


\section{Erkenntnisse zu Fahrradstraßen}

Zur Verkehrssicherheit von Fahrradstraßen lagen bisher nur wenige wissenschaftlich fundierte Untersuchungen aus den Jahren 1997 und 2003 vor. Sie kommen alle zu ähnlichen Ergebnissen: Die Verkehrssicherheit ist positiv zu bewerten. Allerdings kommt es - ähnlich wie in Tempo 30Zonen - zu Überschreitungen der zulässigen Höchstgeschwindigkeit der Kraftfahrzeuge (Kfz) und im Straßenraum parkende oder haltende Kfz werden als eine mögliche Konfliktquelle genannt.

Fahrradstraßen werden bundesweit eingesetzt, rund ein Drittel der 359 befragten Kommunen haben sie bereits im Einsatz. Die Wahrnehmung der Fahrradstraßen in den Kommunen ist überwiegend positiv. Konflikte oder Verkehrssicherheitsdefizite werden selten berichtet. Eine bundesweit einheitliche Gestaltung ist nicht festzustellen; allerdings haben einige Kommunen einheitliche Gestaltungvorgaben festgelegt. Das Befahren und das Parken durch Kraftfahrzeuge ist in fast allen Fahrradstraßen erlaubt.

In Fahrradstraßen ist die Unfallschwere geringer als auf Innerortsstraßen generell. Getötete gab es auf den untersuchten Fahrradstraßen im Untersuchungszeitraum nicht. Der Anteil schwerverletzter Verkehrsteilnehmer in den untersuchten Fahrradstraßen (15\%) liegt leicht unter dem bundesweiten innerörtlichen Durchschnitt (17\%) und entspricht in etwa dem Anteil der schwerverletzten Radfahrer auf Nebenstraßen (14\%). Damit liegt die Unfallschwere in Fahrradstraßen in etwa auf dem Niveau von Nebenstraßen und somit unter dem Durchschnitt des innerörtlichen Gesamtnetzes.

Von den 223 untersuchten Fahrradstraßen waren in 42 Straßen gar keine Radverkehrsunfälle zu verzeichnen. Bei näherer (mikroskopischer) Untersuchung der Radverkehrsunfälle wurde zudem deutlich, dass von den 607 gemeldeten Radverkehrsunfällen nur knapp jeder Dritte einen offensichtlichen Zusammenhang mit der Infrastruktur der Fahrradstraße aufweist. Damit verblieben nur noch 75 Fahrradstraßen mit 186 zu untersuchenden Radverkehrsunfällen in der vertiefenden Untersuchung. Nicht relevante Unfälle wiesen keine Radverkehrsbeteiligung auf, fanden z.B. auf anliegenden Hauptverkehrsstraßen oder auf Privatgrundstücken statt. Auch Unfälle unter Alkoholeinfluss und Alleinunfälle wurden hier nicht gewertet.

Stellt man die Zahl von 223 untersuchten Fahrradstraßen dem Befund von 186 relevanten Radverkehrsunfällen mit Personenschaden in einem Untersuchungszeitraum von 5 Jahren gegenüber, wird deutlich, dass Fahrradstraßen verhältnismäßig sichere Infrastrukturelemente sind.

Vor diesem Hintergrund wurde keine Streckentypisierung, sondern eine Typisierung der Unfälle durchgeführt. Die Typisierung der Unfälle, die einen Zusammenhang mit der Knoten- und Streckengestaltung der Fahrradstraße aufweisen, geben Hinweise auf die Handlungsschwerpunkte bei der Gestaltung von Fahrradstraßen. Zu diesem Zweck wurden alle 186 relevanten Fahrradunfälle nach Strecken- und Knotenunfällen differenziert. Im Rahmen der Unfallanalyse wurden alle 186 relevanten Fahrradunfälle nach Strecken- und Knotenunfällen differenziert, um möglichst konkrete Hinweise auf die Handlungsschwerpunkte bei der Gestaltung von Fahrradstraßen zu erhalten.

Die wenigen Unfälle mit Radverkehrsbeteiligung in Fahrradstraßen fanden zu $35 \%$ in den Zwischenknotenpunkten und zu $34 \%$ auf der Strecke statt. Die Unfälle an den Anfangs- und Endknotenpunkten sowie Bushaltestellen und ähnlichen Sondersituationen hatten einen Anteil von $31 \%$. Diese wurden jedoch mikroanalytisch nicht vertieft untersucht, da die sehr unterschiedliche Gestaltung eine Einzelfallanalyse zur Folge gehabt hätte, die keine Aussagen über diesen Typ insgesamt erlaubt hätte. Der Schwerpunkt der Auswertungen lag somit auf den Zwischenknotenpunkten und den Streckenabschnitten. 
Bei Unfällen mit Radverkehrsbeteiligung in Fahrradstraßen sind Pkw-Fahrer mit Abstand die häufigsten Unfallgegner (76 \% ohne Alleinunfälle Fahrrad, $66 \%$ mit Alleinunfälle Fahrrad). Danach folgen die Alleinunfälle Fahrrad (13\%) und die Fahrrad/Fahrrad-Unfälle (11\%). Die Bedeutung anderer Verkehrsteilnehmer als Unfallgegner ist gering. Pkw sind nicht nur die häufigsten Unfallgegner, sondern auch die häufigsten Unfallverursacher, sowohl in den Zwischenknotenpunkten (77 \% bei den relevanten Unfällen mit Radverkehrsbeteiligung in der Mikroanalyse) als auch auf der Strecke (75 \% bei den relevanten Unfällen mit Radverkehrsbeteiligung in der Mikroanalyse).

Die Analyse von Unfalltyp, Unfallart, Unfallcharakteristik und Unfallursachen hat ergeben, dass an Knotenpunkten Einbiegen-/Kreuzen-Unfälle mit dem Hauptverursacher Kfz dominieren. Die Mikroanalyse verdeutlichte, dass sich dahinter vor allem die Missachtung der Vorfahrtsregelungen durch Kfz verbirgt. Die Dominanz der Einbiegen/Kreuzen-Unfälle gibt deutliche Hinweise auf Sicherheitspotenziale für den Radverkehr an Knotenpunkten in Fahrradstraßen. Demnach ist bei der Planung von Fahrradstraßen auf die Zwischenknotenpunkte ein besonderes Augenmerk zu legen.

Unfälle durch ruhenden Verkehr sind die mit Abstand häufigste Unfallursache auf der Strecke und weisen auf Sicherheitsdefizite für den Radverkehr in Bezug auf das Kfz-Parken hin. Durch die mikroskopische Auswertung der Streckenunfälle wurde deutlich, dass das Längsparken für Radfahrer ein Sicherheitsdefizit darstellt, wenn nicht genügend Abstand zwischen Pkw-Tür und Fahrlinie der Radfahrer eingehalten wird, und dass das Ein- und Ausparken in Querparkständen durch nicht ausreichende Sichtverhältnisse auf den fließenden Verkehr für Radfahrer eine Gefahr darstellt.

Auch Überholvorgänge, die durch Pkw vorgenommen werden, sind bei der insgesamt geringen Unfallbelastung eine häufige Unfallkonstellation. Dabei ist das Überholen bei Gegenverkehr in den Unfallkonstellationen am häufigsten zu erkennen.

Die Verhaltensbeobachtung hat in den untersuchten Fahrradstraßen eine geringe Konflikthäufigkeit aufgezeigt. 98,8 \% aller Interaktionen in Fahrradstraßen verliefen konfliktfrei, die meisten Regelverstöße in Knotenpunkten betrafen die Vorfahrtregelung (86 \%). Dies betraf, wie in der Unfallanalyse, unterschiedliche Vorfahrtregelungen. Allerdings finden sich die typischen Unfallursachen auch in der Art der Konflikte wieder, wie Vorfahrtmissachtung durch kreuzende und einfahrende Kfz. Die zulässige Höchstgeschwindigkeit von $30 \mathrm{~km} / \mathrm{h}$ wurde in den beobachteten Fahrradstraßen von den Kraftfahrzeugen nicht immer eingehalten (im Mittel $V_{85}=36 \mathrm{~km} / \mathrm{h}$ ).

Die Befragung von Verkehrsteilnehmern in Fahrradstraßen hat gezeigt, dass das Wissen über die hier geltenden Verhaltensvorschriften insgesamt sehr lückenhaft ist. Fahrradstraßen werden von $70 \%$ der befragten Verkehrsteilnehmer insgesamt als eine sichere Führungsform genannt. Als Risiken nannten die Befragten die Aspekte „Parkende Kfz“ und „Geschwindigkeit der Kfz“. Es besteht also in diesen Aspekten eine Übereinstimmung zwischen den Befunden der Untersuchung und den Einschätzungen der Nutzer.

\section{Empfehlungen zu Fahrradstraßen:}

Die Fahrradstraße ist eine sichere Führungsform, die auch bei zum Teil sehr hohem Radverkehrsaufkommen eine geringe Unfallbelastung aufweist. Eine weitere Verbesserung der Sicherheit kann über folgende Handlungsschwerpunkte erreicht werden:

- Die Zufahrtserlaubnis für Kraftfahrzeuge sollte grundsätzlich auf Anlieger beschränkt sein, um zusätzliche Probleme mit dem Kfz-Durchgangsverkehr (u.a. vermehrtes Überholen und überhöhte Geschwindigkeiten) zu vermeiden. 
- Für Fahrradstraßen werden Mindestfahrgassenbreiten empfohlen, um ein sicheres Begegnen zu ermöglichen und Unfälle mit parkenden Kfz zu vermeiden. Die entsprechend notwendigen Fahrgassenbreiten lassen sich aus den erforderlichen Lichtraumprofilen der RASt 2006 ableiten. Um das gleichzeitige Begegnen von jeweils zwei nebeneinander fahrenden Radfahrern sicher zu ermöglichen, sollte die Fahrgasse von Fahrradstraßen im Regelfall $4 \mathrm{~m}$ zuzüglich der notwendigen Sicherheitsabstände zu parkenden Fahrzeugen (beim Längsparken 0,75 m) betragen. In diesem Fall sind außerdem ausreichend Sicherheitsabstände zum Überholen eines Radfahrers oder zum Begegnen eines Radfahrers mit einem Pkw vorhanden. Soll gewährleistet werden, dass auch zwei nebeneinander fahrende Radfahrer einem Pkw sicher begegnen können, so ist eine Fahrgassenbreite von mindestens 4,6 m zuzüglich der notwendigen Sicherheitsabstände zu ggf. parkenden Fahrzeugen erforderlich.

- Von deutlich größeren Fahrgassenbreiten ist dagegen abzusehen, da sonst überhöhte Geschwindigkeiten der Kraftfahrzeuge aufgrund vermehrter Überholungen provoziert werden können. Nur, wenn das Befahren der Fahrradstraße für Kraftfahrzeuge nicht erlaubt ist oder das Überholen bereits durch hohe Radverkehrsstärken weitgehend unterbunden wird, können auch größere Fahrgassenbreiten Anwendung finden.

- Die Sicherheitstrennstreifen sollten durch Markierungen gekennzeichnet werden, ggf. unterstützt durch Piktogramme, die den Fahrweg des Radfahrers kennzeichnen.

- Die Vorfahrt im Zuge einer Fahrradstraße sollte einheitlich geregelt sein. Damit die Fahrradstraße auch ihrer Bedeutung als Infrastrukturelement mit Vorrang für Radfahrer gerecht wird, sollte möglichst eine Vorfahrt auf der Fahrradstraße angestrebt werden. Die Unterordnung der Nebenzufahrten muss dabei jeweils eindeutig erkennbar sein, z.B. durch Beschilderung, Aufpflasterung, abgesenkte Bordsteine, Einengungen o.ä.

- Für die Gestaltung von Knotenpunkten sollten bundesweit einheitliche Gestaltungsprinzipien angewendet werden.

- Neben der Beachtung der planerischen Aspekte werden polizeiliche Kontrollen zum regelkonformen Verhalten sowie eine entsprechende Aufklärungsarbeit zu den Verkehrsregeln in Fahrradstraßen empfohlen.

- Die Aufnahme des Infrastrukturelements „Fahrradstraße“ bei der Unfallaufnahme würde die Unfallanalyse und damit ein Monitoring der weiteren Entwicklung deutlich vereinfachen.

\section{Erkenntnisse zu in Gegenrichtung für Radverkehr geöffneten Einbahnstraßen}

Die aktuellsten umfassenden Untersuchungen zu geöffneten Einbahnstraßen stammen aus den Jahren 1997 und 2001. Darin wird ausgeführt, dass geöffnete Einbahnstraßen im Vergleich zu nicht geöffneten Einbahnstraßen nicht unfallauffälliger sind, sondern einen positiven Einfluss auf die Verkehrssicherheit und den Verkehrsablauf haben. Wichtige Erkenntnisse waren, dass Kfz bei Begegnungen mit Radfahrern deutlich langsamer als bei unbehinderter Fahrt fahren, beim Überholen in gleicher Fahrtrichtung kommt es dagegen zu einer Beschleunigung. Auch konnte festgestellt werden, dass durch die Öffnung der Einbahnstraße Sicherheitsgewinne vor allem für Fußgänger zu verzeichnen sind, da Radfahrer nun auf der Fahrbahn fahren und nicht mehr auf dem Gehweg Fußgänger gefährden. Es gibt aber auch Hinweise, dass geöffnete Einbahnstraßen vor allem von Gelegenheitsradfahrern kritisch gesehen werden.

Die Öffnung von Einbahnstraßen für den Radverkehr in Gegenrichtung ist ein weit verbreitetes Element. Mehr als $80 \%$ der 359 an der Befragung teilnehmenden Kommunen setzen dieses ein. 
Von den Kommunen wird die Öffnung der Einbahnstraßen als weitgehend sicher eingeschätzt. Lediglich $1 \%$ aller genannten Einbahnstraßen wurde als problematisch benannt.

In nur $36 \%$ der Unfälle in als problematisch genannten geöffneten Einbahnstraßen fanden Unfälle mit Radverkehr in Gegenrichtung statt. In der Kölner Innenstadt waren dies sogar nur $22 \%$ der Radverkehrsunfälle. Für die Kölner Innenstadt heißt das konkret, dass in den 200 geöffneten Einbahnstraßen pro Jahr nur insgesamt ca. 13 Unfälle mit Radfahrern in Gegenrichtung polizeilich registriert wurden. Somit hat die Sonderuntersuchung in der Kölner Innenstadt ergeben, dass auch wenn alle geöffneten Einbahnstraßen in einem definierten Raum untersucht werden, nur wenige geöffnete Einbahnstraßen Unfälle mit Radfahrern in Gegenrichtung aufweisen.

Die Schwere der Radverkehrsunfälle in geöffneten Einbahnstraßen ist mit 9 \% Schwerverletzten deutlich geringer als im bundesweiten innerörtlichen Durchschnitt (17\% Schwerverletzte).

Betrachtet man nur den Anteil der Unfälle, die sich mit Radfahrern ereignen, die in der Einbahnstraße in Gegenrichtung unterwegs sind, ist dieser noch einmal geringer (4 \% bzw. $6 \%$ Schwerverletzte). Auch bei den Einbahnstraßen, die für den Radverkehr in Gegenrichtung geöffnet sind, findet die Mehrzahl der Unfälle mit Radverkehrsbeteiligung an Knotenpunkten statt. In für den Radverkehr geöffneten Einbahnstraßen überwiegen Einbiegen/Kreuzen-Unfälle (57\%) bei den Unfällen mit Radfahrern in Gegenrichtung stark.

Eine Auffälligkeit der Untersuchung in der Kölner Innenstadt war der hohe Anteil von Überschreiten-Unfällen (30 \%). Die mikroskopische Unfalluntersuchung konnte zeigen, dass diese Überschreiten-Unfälle in geöffneten Einbahnstraßen vor allem in den Haupteinkaufsstraßen der KöIner Innenstadt mit hoher Fußgängerfrequenz passieren. Hier achten Fußgänger beim Überqueren der Straße oft nicht auf den aus entgegen der allgemeinen Fahrtrichtung kommenden Fahrradverkehr.

Der Pkw ist in den bundesweit untersuchten geöffneten Einbahnstraßen bei den insgesamt wenigen Unfällen der häufigste Unfallgegner (65\%) und der häufigste Hauptverursacher (65 \%). In der Kölner Innenstadt sind sowohl Radfahrer (40\%), Pkw-Fahrer (32\%) als auch Fußgänger (27 \%) häufige Unfallverursacher. Fußgänger verursachen hier ausschließlich Unfälle auf der Strecke.

Die Videobeobachtung in fünf ausgewählten geöffneten Einbahnstraßen hat eine sehr geringe Konflikthäufigkeit zwischen in Gegenrichtung fahrenden Radfahrern und anderen Verkehrsteilnehmern ergeben. Mehr als $97 \%$ aller Interaktionen sind konfliktfrei verlaufen, die beobachteten Konflikte betreffen Vorfahrtmissachtungen sowie die falsche Fahrbahnnutzung.

\section{Empfehlungen zu geöffneten Einbahnstraßen:}

In der Regel gestaltet sich die Öffnung von Einbahnstraßen für den Radverkehr unproblematisch, so dass auf besondere Maßnahmen verzichtet werden kann. Bei der Freigabe des Radfahrens in Gegenrichtung ist jedoch auf die Einhaltung der Vorgaben zur Öffnung von Einbahnstraßen gemäß VwV-StVO und den RASt 06 zu achten. Das betrifft vor allem die Beschilderung an den Knotenpunkten mit dem Zusatzzeichen „Radverkehr aus beiden Richtungen“. Falls sich in geöffneten Einbahnstraßen an Knotenpunkten Verkehrssicherheitsprobleme abzeichnen, sollte geprüft werden, ob die in den ERA oder RASt empfohlenen unterstützenden Markierungen und Beschilderungen erfolgen können, wie z. B. die Anordnung von Gefahrenzeichen 102 „Kreuzung oder Einmündung mit Vorfahrt von rechts“ mit dem Zusatzzeichen 1000-30 (,beide Richtungen“) oder 101 „Gefahrstelle“ bzw. 138 „Radfahrer kreuzen“, mit dem Zusatzzeichen 1000-32 („Radverkehr aus beiden Richtungen“), wie es in den ERA bzw. den RASt empfohlen wird. 
In Geschäftsstraßen mit sehr hohem Fußgängeraufkommen ist auch den Fußgängern die Einbahnstraße zu verdeutlichen, da Fußgänger die aus der Gegenrichtung kommenden Radfahrer häufig nicht wahrnehmen:

- Markierungen in Form von Piktogrammen und Pfeilen

- Neuordnung des Parkens, um notwendige Sichtfelder freizuhalten.

In geöffneten Einbahnstraßen sollte bei der Unfalldatenaufnahme auch die Fahrtrichtung mit erfasst werden.

\section{Resümee}

Beide Infrastrukturelemente sind als verhältnismäßig verkehrssicher einzustufen. Es hat sich gezeigt, dass das Unfallgeschehen insgesamt wie auch dasjenige mit Radfahrerbeteiligung sich auf einem sehr niedrigen Niveau bewegt. Der Einsatz der beiden Elemente kann somit empfohlen werden. Trotz des grundsätzlich sehr hohen Niveaus der Verkehrssicherheit konnten auf Basis der Untersuchungen für beide Elemente weitergehende gestalterische und ordnungsrechtliche Hinweise für die kommunale Praxis formuliert werden. 


\begin{abstract}
Cycling roads and one-way streets where cycling against the flow of traffic is permitted are now part of the standard repertoire of cycling traffic planners in Germany. These two infrastructure elements were introduced in Germany in 1997 by an amendment of the German Road Traffic Regulations (StVO). However, they differ in terms of their intended purpose and the information available about their safety. The purpose of permitting contra-flow cycling on one-way streets is, above all, to increase the use of minor roads by cyclists and minimize the diversions they have to take. The safety of one-way streets with contra-flow cycling has been studied in depth, and their essential safety has been demonstrated. Cycling roads, on the other hand, are intended primarily to attract cyclists and give them priority on roads that are particularly suitable for cycling. These roads should be introduced, above all, where there is a high density of cyclists. Whereas cycling roads spread fairly slowly in the early years following their introduction, in recent years they have spread more quickly. In contrast to one-way streets with contra-flow cycling, the safety of dedicated cycling roads has not yet been studied extensively.
\end{abstract}

\title{
Aim
}

The planning offices Planerbüro Südstadt and VIA were commissioned by the UDV to carry out a research project to obtain new findings on the spread, areas of application and road safety of these two infrastructure elements. Since there are established findings on the road safety of contra-flow cycling on one-way streets, the main focus of the study was cycling roads. The analysis of one-way streets was restricted to streets with contra-flow cycling that had a conspicuously high incidence of accidents.

\section{Methodology}

The studies of the two infrastructure elements were carried out separately, although they both followed the same pattern. Following an analysis of the literature, a Germany-wide online survey was conducted of 359 municipalities of different sizes in order to gain an overview of the spread, layout, design and operation of these two elements in practice at the local level. Concrete examples were requested in order to obtain sections of road that could be used in the study. The accident data of the years 2008 to 2012 for the 177 cycling roads and 31 one-way streets with contra-flow cycling that were mentioned by the municipalities as being problematic was analyzed macroscopically ${ }^{1}$ and microscopically ${ }^{2}$ and related to the infrastructure in place locally. Based on the results of the survey of municipalities and the accident analysis, 26 locations were selected for behavioral observation, 21 of which were on cycling roads and five on one-way streets with contraflow cycling. At 21 intersections and on five sections of road, data on traffic and behavior (traffic volumes, speeds, interactions and conflicts) was obtained and analyzed. In addition, cyclists, drivers and pedestrians (a total of 452 road users) were surveyed locally about their knowledge of the rules on cycling roads and how safe they felt. Finally, recommendations for the safe design of cycling roads and one-way streets with contra-flow cycling were obtained on the basis of the findings.

\section{Findings for cycling roads}

\footnotetext{
${ }^{1} 607$ cycling accidents involving injury on cycling roads and 152 on one-way streets with contra-flow cycling

${ }^{2} 186$ accidents involving cyclists and injury on 75 cycling roads and 54 accidents involving contra-flow cycling and injury on 20 one-way streets with contra-flow cycling
} 
In accordance with the General Administrative Regulations on the Road Traffic Regulations ( $V w V-S t V O)$, cycling roads can be established where cycling is the predominant form of transport or is expected to be soon. The volume of motor traffic on cycling roads must be low. Cycling roads are designated by means of traffic signs 244.1 and 244.2 of the StVO. Any other traffic than cycling traffic may only exceptionally be permitted provided relevant additional signs are in place (permitting residents' vehicles only, for example). The speed limit on cycling roads is $30 \mathrm{~km} / \mathrm{h}$. Cycling traffic on cycling roads must not be put in danger or hindered by other kinds of traffic. Motor vehicles must reduce their speed further wherever necessary. It is permitted for cyclists to cycle side by side. The stipulations regarding right of way at the intersections of cycling roads in the guidelines on the design of roads in built-up areas (RASt 2006) and in the recommendations for cycling facilities (ERA 2010) are different. Whereas RASt 2006 stipulates that cycling roads should have right of way over other local access roads, in ERA 2010 it is recommended that this should depend on the local

The studies of road safety on cycling roads examined in the analysis of the literature are based on very small samples and do not allow generally applicable conclusions to be drawn. However, it became apparent that the cycling roads previously studied did not have a conspicuously high number of conflicts or accidents.

According to the survey of municipalities, cycling roads have been implemented throughout Germany, and the municipalities' assessment of their safety is overwhelmingly positive. Although, according to VwV-StVO, motor traffic is only permitted on cycling roads in exceptional cases, motor vehicles are permitted to use virtually all the cycling roads studied (96 percent), and in almost twothirds of them this is not limited to residents only. Moreover, the cycling roads studied were neither uniformly designed nor did they have uniform arrangements governing right of way.

The accident analysis showed that cycling roads are essentially relatively safe. An average of only three to four accidents involving cyclists and injury occurred per cycling road in the five-year period studied. Moreover, only around one in three of these accidents had an evident connection with the cycling road. Around half of the accidents happened on an open section of road and half at an intersection. Despite the low total number of accidents, they followed a typical pattern. 80 percent of the accidents involving cyclists at intersections were "turning-into/crossing" accidents (where vehicles failed to give way and turned into or crossed the road). 56 percent of the accidents on the open sections of road occurred in connection with the parking of motor vehicles, and 19 percent occurred during overtaking with the involvement of motor vehicles. The most common other party in an accident involving a cyclist was a car (in 76 percent of cases). In three of every four accidents, the driver of the car was the main cause of the accident.

99 percent of the roughly 6,500 interactions between road users on cycling roads were found to be free of conflict in the behavioral observation section of the study. Most of the 80 conflicts identified concerned right of way (76 percent).

Motor vehicles did not always keep to the speed limit of $30 \mathrm{~km} / \mathrm{h}$ on the observed cycling roads $\left(\mathrm{V}_{85}=36 \mathrm{~km} / \mathrm{h}\right.$ on average). Excessive speeds were found more often on wide cycling roads where there was also a low proportion of cycling traffic $\left(V_{85}=38 \mathrm{~km} / \mathrm{h}\right)$. More than one in three motor vehicles were driving faster than $35 \mathrm{~km} / \mathrm{h}$ (34 percent), and around one in 12 were driving faster than $40 \mathrm{~km} / \mathrm{h}$ (8 percent) on wide cycling roads.

The results of the survey of road users showed large gaps in the knowledge of all road users with regard to the rules on cycling roads. Virtually no differences were found in this respect between cyclists, pedestrians and drivers. For example, three-quarters of those surveyed did not know that other vehicles may only use cycling roads when it is expressly permitted by means of an additional road sign. Only around half of those surveyed knew that cyclists on cycling roads are allowed to 
cycle side by side or that cyclists do not always have right of way at intersections on cycling roads. Around one in three road users did not know that cyclists also have to cycle on the right on cycling roads or that the speed limit is $30 \mathrm{~km} / \mathrm{h}$. In addition, the cycling roads observed were clearly not always recognized as such by all road users. For example, around one in four of the road users surveyed were not aware that they were on a cycling road when surveyed (in the case of drivers it was one in three).

\section{Recommendations for cycling roads}

As stipulated in VwV-StVO and the relevant design guidelines, cycling roads come into consideration where cycling is the most common form of transport or is expected to be before long. The volume of motor traffic on cycling roads must be low according to VwV-StVO, and motor traffic may only be permitted in exceptional cases. A cycling road should therefore only be created provided these criteria are met. A certain absolutely necessary but significantly lower level of motor traffic than usual (e.g. residents only) can be tolerated provided the conditions specified below are met.

Cycling roads are essentially safe because accidents are relatively rare. Nevertheless the great majority of cycling accidents on cycling roads involve motor vehicles, despite the fact that they are only supposed to be permitted on cycling roads in exceptional cases. However, during the study it was found that motor vehicles are permitted to use virtually all the cycling roads studied, and in almost two-thirds of them this is not limited to residents only. It is recommended that there should be a more restrictive approach to allowing motor vehicles to use these roads. That means that VwV-StVO must be applied rigorously in this respect. Wherever possible, the unlimited permission for motor vehicles to use cycling roads should not be given. If it is absolutely necessary in order to provide access, it should be permitted for residents only. This also means that, before a cycling road is established, it must be examined whether there are suitable alternative routes available for the motor vehicles that are to be excluded. In addition, the ban on motor vehicles without permission entering or passing through cycling roads must be policed, and offending drivers must be penalized.

Although the research project did not investigate direct relationships between road width and accidents, recommendations for road widths of bicycle roads can be derived from the results of the investigations. For accidents on open sections of cycling roads, two causes of accidents are the most important: accidents in connection with the parking of motor vehicles and accidents occurring during overtaking with the involvement of motor vehicles. Accidents with parked motor vehicles occur primarily on narrow cycling roads. Accidents during overtaking were more likely to be found on wider cycling roads. Consequently, the width of the road plays an important role in road safety on cycling roads, particularly when motor vehicles are permitted. The suitable road design can be derived from the necessary clearance profiles of the RASt 2006. In order to ensure that two cyclists cycling side-by-side can pass two oncoming cyclists cycling side-by-side safely, the width of the cycling road consequently should be at least $4 \mathrm{~m}$ plus the required safety clearance from parked vehicles ( $0.75 \mathrm{~m}$ in the case of parallel parking). In this case, it is also possible for cars to overtake or pass oncoming single cyclists while maintaining the required safety clearance. To ensure that two cyclists cycling side-by-side can pass an oncoming car safely, a road with of at least $4.6 \mathrm{~m}$ plus the required safety clearance from parked vehicles is necessary.

Significantly wider roads are not to be recommended, since otherwise drivers would be tempted to drive at excessive speeds because the wider road offers them more scope to overtake. Greater road widths may be used only when motor vehicles are not permitted on the cycling road or overtaking is largely prevented by high volumes of cycling traffic. 
In order to make the safety clearance from parked vehicles clear, a safety line can be painted on the road surface next to the parked vehicles $(0.75 \mathrm{~m}$ in the case of parallel parking). In addition to the prescribed traffic signs, it can be useful to mark the pictogram "bicycle" or the traffic sign 244.1 - "bicycle road" on the road, in order to clarify the road users once again that they are in a bicycle road.

In addition, wherever possible, a cycling road should have a uniform design along its entire length. That applies, above all, to the rules on right of way at intersections. Wherever possible, cycling traffic should have right of way at intersections, except at intersections with main roads, to ensure that the cycling road fulfills the intention of being an infrastructure element that gives cyclists priority. It must be clear in each case that traffic from side roads must give way by means of signs, humps, lowered curbs or narrowing of the roadway, for example.

If it is not possible for traffic-related or structural reasons, for example, to give a cycling road right of way at the overwhelming majority of its intersections, it is questionable whether it makes sense to create a cycling road in this case. The study revealed that there is not much difference between cycling roads and roads in $30 \mathrm{~km} / \mathrm{h}$ zones in terms of the accidents that occur. The signs on cycling roads that were previously roads in $30 \mathrm{~km} / \mathrm{h}$ zones and where the right-before-left rule is still applicable at intersections are therefore questionable, at least from the point of view of road safety, but also given that the intention of creating a cycling road is to prioritize cycling.

As the results of the project show, not only is compliance with the planning aspects mentioned above required, but police checks are also necessary to ensure compliance with the rules, and work has to be done to explain the rules applicable on cycling roads.

\section{Findings for one-way streets with contra-flow cycling}

In a study of the German Federal Highway Research Institute (BASt), it was found that opening one-way streets to contra-flow cycling has a positive impact on road safety on one-way streets (PGV/BIS 2001). Drivers drive more slowly on one-way streets when there are oncoming cyclists, whereas they generally accelerate when they are overtaking cyclists. It was also found that pedestrian safety is improved. Conflicts with cyclists cycling illegally on the pavement against the flow of traffic are avoided, because once the one-way street is open to contra-flow cycling, the cyclists generally cycle legally on the roadway.

Within the framework of the current UDV study, a Germany-wide survey of municipalities revealed that one-way streets with contra-flow cycling are very widespread throughout the country. 84 percent of the municipalities surveyed (260 of 311 municipalities) indicated that they had opened a total of 2,373 one-way streets to contra-flow cycling. Only 25 of these one-way streets with contra-flow cycling (1 percent) were rated problematic in terms of road safety by the municipalities.

Moreover, the accident analysis carried out for one-way streets with contra-flow cycling that were rated problematic indicated that only around one in three cycling accidents on these one-way streets involved cyclists cycling against the flow of traffic. In addition, the analysis of accidents in a study area in the center of Cologne used for comparison purposes confirmed that there are very few accidents involving cyclists cycling against the flow on one-way streets with contra-flow cycling. In a total of 200 one-way streets with contra-flow cycling in the center of Cologne, an average of around only 13 accidents a year occurred involving cyclists cycling against the flow of traffic.

Where accidents involving contra-flow cyclists occurred at intersections, these were typically "turning-into/crossing" accidents (accidents in which vehicles failed to give way and turned into or crossed the road, which accounted for over 70 percent of the accidents involving contra-flow 
cyclists and injury). On sections of road without intersections, accidents involving pedestrians crossing the road occurred, above all, in shopping streets, where pedestrians more often tend to cross the road.

In the observations of behavior on five one-way streets with contra-flow cycling, very few conflicts with contra-flow cyclists were observed. There were 12 conflicts in 475 observed interactions, a conflict rate of around 2.5 percent. The conflicts occurred as a result of failures to give way (eight conflicts) or incorrect use of the roadway (four conflicts).

\section{Recommendations for one-way streets with contra-flow cycling}

One-way streets with contra-flow cycling are essentially very safe. When contra-flow cycling is to be permitted on a one-way street, the relevant guidelines for one-way streets with contra-flow cycling in VwV-StVO and RASt 06 should be complied with. That means, above all, that there should be signs at intersections indicating that there is cycling traffic in both directions. In shopping streets where pedestrians frequently cross the road, the fact that contra-flow cycling is permitted should also be made clear to pedestrians (e.g. by means of pictograms and arrows on the road surface). At the corresponding crossing points, it must be ensured that pedestrians have an adequate view of any contra-flow cyclists. It may be necessary to make different arrangements for parking in order to make sure that contra-flow cyclists are within pedestrians' field of vision. 


\section{Forschungsgegenstand}

Der Radverkehr wurde in den letzten Jahren von vielen Kommunen gezielt gefördert. Der Anteil des Radverkehrs hat stetig zugenommen und wird voraussichtlich weiter steigen. Wenn dies auch Entscheidungen der Verkehrsteilnehmer sind, die auf den unterschiedlichsten Motiven beruhen, hat die mittlerweile langjährige Radverkehrsförderung einen Beitrag dazu geleistet. Eine wesentliche Komponente der Radverkehrsförderung ist die Entwicklung sicherer, komfortabler und durchgängiger Radverkehrsnetze. Die Netzelemente „Fahrradstraße“ und die „Öffnung von Einbahnstraßen für den Radverkehr in Gegenrichtung“ gehören dabei zum festen Repertoire der Radverkehrsinfrastruktur.

In Fahrradstraßen ist nach VwV-StVO das Fahrrad die vorherrschende Verkehrsart ${ }^{3}$. Andere Fahrzeuge dürfen nur ausnahmsweise zugelassen werden. Die in Fahrradstraßen geltenden Regeln, der besonderen Rücksichtnahme gegenüber dem Radverkehr und, dass Radfahrer hier nebeneinander fahren dürfen, scheinen jedoch bei den Verkehrsteilnehmern nicht sehr bekannt zu sein. Auch liegen bislang keine Erkenntnisse darüber vor, ob und wie sich Fahrradstraßen auf die Verkehrssicherheit auswirken. Zudem bestehen derzeit keine Vorgaben, unter welchen Voraussetzungen Fahrradstraßen notwendig oder sinnvoll sind und ob sie bestimmte Gestaltungselemente aufweisen sollten.

Fahrradstraßen werden in den letzten Jahren vermehrt eingesetzt. In vielen Kommunen (u.a. München, Köln, Bonn) werden in großem Umfang Fahrradstraßen eingesetzt. Neue Fahrradstraßen werden vor allem im Zuge städtischer Fahrradrouten angeordnet, die dem Radverkehr ein durchgängiges und komfortables Netz abseits der Hauptverkehrsstraßen anbieten sollen. Durch die in neuester Zeit vermehrte Planung von Radschnellverbindungen, ist ein neues Anwendungsgebiet geschaffen worden, das voraussichtlich zur Einrichtung weiterer Fahrradstraßen führen wird.

Die Öffnung von Einbahnstraßen für den Radverkehr in Gegenrichtung ist hingegen an bestimmte Einsatzkriterien (VwV-StVO) ${ }^{4}$ gekoppelt und wird in vielen Kommunen seit Jahren praktiziert. Es gibt nur wenige Kommunen, die das Element nicht einsetzen. Hinsichtlich der geöffneten Einbahnstraßen gilt es deshalb, die Erfahrungen der Kommunen nach über zehn Jahren zu dokumentieren.

Beide Netzelemente haben gemeinsam, dass sie einen eher geringen baulichen Aufwand erfordern. Die Anordnung obliegt der Straßenverkehrsbehörde, die in enger Abstimmung mit der Polizei vor allem die Aspekte der Verkehrssicherheit im Auge behalten muss.

Hinsichtlich des Forschungsstandes und der Umsetzung unterscheiden sich beide Netzelemente jedoch erheblich:

3 VwV-StVO (2009): Fahrradstraßen kommen dann in Betracht, wenn der Radverkehr die vorherrschende Verkehrsart ist oder dies alsbald zu erwarten ist. Absatz 1, Abschnitt I.

4 VwV-StVO (2009): 1. Beträgt in Einbahnstraßen die zulässige Höchstgeschwindigkeit nicht mehr als 30 $\mathrm{km} / \mathrm{h}$, kann Radverkehr in Gegenrichtung zugelassen werden, wenn

5 a) eine ausreichende Begegnungsbreite vorhanden ist, ausgenommen an kurzen Engstellen; bei Linienbusverkehr oder bei stärkerem Verkehr mit Lastkraftwagen muss diese mindestens 3,5 $\mathrm{m}$ betragen, 6 b) die Verkehrsführung im Streckenverlauf sowie an Kreuzungen und Einmündungen übersichtlich ist, 7 c) für den Radverkehr dort, wo es orts- und verkehrsbezogen erforderlich ist, ein Schutzraum angelegt wird. Absatz 4, Abschnitt IV. 
- Seit der StVO-Novelle vom 1. September 1997 sind bereits sehr viele Einbahnstraßen geöffnet worden. Für den Fragenkomplex zur Öffnung von Einbahnstraßen liegt seit 2002 (PGV/BIS: 2001) eine aussagekräftige und belastbare Studie vor.

- Zu Fahrradstraßen, die ebenfalls seit der StVO-Novelle vom 1. September 1997 angeordnet werden dürfen, liegen dagegen keine belastbaren Erkenntnisse vor.

\section{Ziel des Forschungsvorhabens}

Das Hauptinteresse bei den Fahrradstraßen besteht also darin, einen bundesweiten Überblick über die Verbreitung, Gestaltung und Aspekte der Verkehrssicherheit zu bekommen. Dabei stehen Aspekte der Verkehrssicherheit im Vordergrund. So sollten folgende Fragen beantwortet werden:

- Wie ist es um die Kenntnis des Netzelements Fahrradstraße bei Planern und Nutzern bestellt?

- Wie wird das Element Fahrradstraße von Planern und Nutzern bewertet?

- Wie stellt sich das Unfallgeschehen im Vergleich zum übrigen Straßennetz (innerorts) dar?

- Lassen sich bestimmte Muster bezogen auf das Unfallgeschehen identifizieren, die für Fahrradstraßen typisch sind?

- Sind innerhalb der untersuchten Fahrradstraßen Unterschiede im Unfallgeschehen und in der Konfliktdichte erkennbar?

- Lassen sich Interpretationsansätze für diese Unterschiede finden, die auf die Gestaltung und Charakteristik der Fahrradstraßen zurückzuführen sind?

- Besteht möglicherweise weiterer Forschungsbedarf hinsichtlich Optimierung sowohl der Verkehrsregelungen als auch der Gestaltung von Fahrradstraßen?

Bei den für Radverkehr in Gegenrichtung geöffneten Einbahnstraßen lag der Schwerpunkt auf einer Aktualisierung der bereits vorliegenden Aussagen. Dabei wurde der Schwerpunkt auf die Untersuchung unfallauffälliger Einbahnstraßen gelegt. Gleichwohl sollte die stichprobenhafte Betrachtung unfallträchtiger Einbahnstraßen durch eine ergänzende, flächenhafte Untersuchung in der Kölner Innenstadt ergänzt werden, um eine einseitige Fokussierung auf Problemfälle zu vermeiden.

Das Ziel der Untersuchung war es, Empfehlungen zum Einsatz der Netzelemente „Fahrradstraße“ und „Öffnung von Einbahnstraßen“ unter besonderer Berücksichtigung ihrer Auswirkungen auf die Verkehrssicherheit zu erarbeiten. 


\section{Methodik}

Da die Ausgangslage bei Fahrradstraßen und für den Radverkehr in Gegenrichtung geöffnete Einbahnstraßen unterschiedlich war, sind die beiden Themenkomplexe weitgehend getrennt voneinander bearbeitet worden. Eine Übersicht des Projektablaufes wird in Abbildung 1 gegeben.

\begin{tabular}{|c|c|c|c|}
\hline \multicolumn{2}{|c|}{ Fahrradstraßen } & \multicolumn{2}{|c|}{ Geöffnete Einbahnstraßen } \\
\hline \multicolumn{4}{|c|}{ Literaturanalyse } \\
\hline \multicolumn{4}{|c|}{ Kommunalbefragung } \\
\hline \multicolumn{2}{|c|}{ Kommunen Phase I $(n=359)$} & \multicolumn{2}{|c|}{ Kommunen Phase I $(n=359)$} \\
\hline \multicolumn{2}{|c|}{$\begin{array}{l}\text { Fahrradstraßen }(n=426) \text {, } \\
\text { Kommunen mit Fahrradstraßen } \\
\qquad(n=110)\end{array}$} & \multicolumn{2}{|c|}{$\begin{array}{l}\text { Geöffnete Einbahnstraßen } \\
\qquad(n=2.373) \text {, davon als } \\
\text { problematisch benannt }(n=25)\end{array}$} \\
\hline \multicolumn{2}{|c|}{ Kommunen Phase II $(n=60)$} & \multicolumn{2}{|c|}{ Kommunen Phase II $(n=10)$} \\
\hline \multicolumn{2}{|c|}{$\begin{array}{l}\text { Namentlich genannte } \\
\text { Fahrradstraßen }(n=179)\end{array}$} & \multicolumn{2}{|c|}{$\begin{array}{l}\text { Namentlich genannte geöffnete } \\
\text { Einbahnstraßen }(n=17)\end{array}$} \\
\hline \multicolumn{4}{|c|}{ Makroskopische Unfallanalyse } \\
\hline \multicolumn{2}{|c|}{ Fahrradstraßen $(n=181)$} & \multicolumn{2}{|c|}{$\begin{array}{c}\text { Problematische geöffnele } \\
\text { Einbahnstraßen }(n=18)\end{array}$} \\
\hline $\begin{array}{l}\text { UDV-EUSKA- } \\
\text { Unfalldaten } \\
\text { Nebenstraßen } \\
\quad(n=101)\end{array}$ & $\begin{array}{l}\text { Destatis } \\
\text { Unfalldaten } \\
\text { bundesweit, } \\
\text { innerorts }\end{array}$ & $\begin{array}{l}\text { Einbahnstraßen- } \\
\text { Unfälle Köln } \\
\text { Innenstadt }\end{array}$ & $\begin{array}{l}\text { Destatis } \\
\text { Unfalldaten } \\
\text { bundesweit, } \\
\text { innerorts }\end{array}$ \\
\hline \multicolumn{4}{|c|}{ Mikroskopische Unfallanalyse } \\
\hline \multicolumn{2}{|c|}{ Fahrradstraßen ( $n=177)$} & \multicolumn{2}{|c|}{$\begin{array}{c}\text { Problematische geöffriete } \\
\text { Einbahnstraßen }(n=31)\end{array}$} \\
\hline \multicolumn{4}{|c|}{ Videobeobachtung (26 Standorte) } \\
\hline \multicolumn{2}{|c|}{ Fahrradstraßen (n=21) } & \multicolumn{2}{|c|}{ Geöffnete Einbahnstraßen ( $n=5$ ) } \\
\hline $\begin{array}{l}\text { Knoten }(n=17) \\
\text { - Interaktion } \\
\text { - Verkehrsmenge }\end{array}$ & $\begin{array}{l}\text { Strecken }(n=4) \\
\text { - Interaktion } \\
\text { - Verkehrsmenge } \\
\text { - Geschwindigkeit }\end{array}$ & $\begin{array}{l}\text { Knoten ( } n=4) \\
\text { - Interaktion } \\
\text { - Verkehrsmenge }\end{array}$ & $\begin{aligned} &\text { Strecken ( } n=1) \\
& \text { + interaktion } \\
& \text { - Verkehrsmenge } \\
& \text { - Geschwindigkeit }\end{aligned}$ \\
\hline \multicolumn{2}{|c|}{ Befragung } & & \\
\hline \multicolumn{2}{|c|}{$\begin{array}{c}\text { Verkehrsteilnehmer }(n=452 \text {, } \\
10 \text { Fahrradstraßen) }\end{array}$} & & \\
\hline \multicolumn{4}{|c|}{ Empfehlungen } \\
\hline Fahrra & straßen & Geōffnete Ei & ibahnstraßen \\
\hline
\end{tabular}

Abbildung 1: $\quad$ Ablauf des Forschungsprojektes 


\section{$2.1 \quad$ Literaturauswertung}

Es wurde eine Literaturanalyse durchgeführt, die den aktuellen Stand zu den Themen „Fahrradstraßen“ und „Öffnung von Einbahnstraßen“ in deutsch- und englischsprachiger Literatur aus den Niederlanden, Österreich und Deutschland dokumentiert. Gesichtet wurden Evaluationen, Aufsätze und (kommunale) Untersuchungen, die nach 1994 veröffentlicht wurden. Dabei wurde zum einen die vorliegende offizielle Literatur analysiert und bewertet, zum anderen aber auch nach sog. „grauer“ Literatur (Diplomarbeiten, etc.) recherchiert. Ebenfalls wurden die entsprechenden Vorschriften und Planungsrichtlinien nach Festlegungen zu den beiden Infrastrukturelementen ausgewertet.

Das Ziel war, die Einsatzbereiche der beiden Infrastrukturelemente zu erfassen, den Umsetzungsstand zu beschreiben und die Erfahrungen vor allem in Hinblick auf die Auswirkungen auf die Verkehrssicherheit zusammenzufassen.

\subsection{Bundesweite kommunale Umfrage}

Im November 2013 erfolgte als zweiter Arbeitsschritt eine deutschlandweite Befragung von Kommunen zum aktuellen Stand des Einsatzes beider Radverkehrselemente. Es wurde erfragt, wie häufig und unter welchen Rahmenbedingungen die beiden Infrastrukturelemente eingesetzt werden. Allgemeine Informationen über die Kommunen ergänzten die Befragung. Im Fokus stand auch die (subjektive) Bewertung der beiden Infrastrukturelemente durch die Vertreter der Kommune. Es wurden Details zu einzelnen Fahrradstraßen und den als problematisch eingeschätzten geöffneten Einbahnstraßen erhoben. Die Ergebnisse flossen in die Auswahl der weiter zu untersuchenden Straßenräume ein.

Mithilfe der Erhebung sollten mehrere Forschungsfragen beantwortet werden:

- Gibt es räumliche Unterschiede bei der Verbreitung von Fahrradstraßen und geöffneten Einbahnstraßen?

- Gibt es Unterschiede in der Gestaltung oder Verkehrsregelung?

- Wie schätzen die Kommunen die Verkehrssicherheit in den Fahrradstraßen und geöffneten Einbahnstraßen in ihrer Kommune subjektiv ein?

- Können in geöffneten Einbahnstraßen Sicherheitsdefizite identifiziert werden?

Die Befragung war zweistufig aufgebaut. In der ersten Phase wurden 1.076 Kommunen zum allgemeinen Einsatz von Fahrradstraßen und geöffneten Einbahnstraßen befragt. Die Kommunen, die Fahrradstraßen aufweisen, wurden in der zweiten Befragungsphase um Details zu den Fahrradstraßen, wie z.B. Verkehrsstärken, Netzbedeutsamkeit für den Radverkehr und dem Zeitpunkt der Einrichtung gebeten. Im Falle der geöffneten Einbahnstraßen wurden nur von den Straßen Detailangaben angefragt, bei denen die Kommunen die Situation als problematisch / unfallauffällig einschätzen. In diesen Fällen wurde auch um nähere Angaben zu den geöffneten Einbahnstraßen gebeten, um die Problemlage bewerten zu können.

In beiden Fällen wurden die Kommunen um die Bereitstellung von Fotodateien und Lageplänen etc. gebeten, um einen visuellen Eindruck der jeweiligen Straßenräume zu erhalten.

Beide Befragungen wurden als Online-Befragung durchgeführt.

\section{Phase I der Befragung}

Die Befragung wurde bundesweit in einer geschichteten Stichprobe durchgeführt. Die Schichtung wurde aufgrund der Einwohnerzahl der Kommunen vorgenommen. 
- Bei den Kommunen mit mehr als 20.000 Einwohnern wurde eine Vollerhebung durchgeführt, in den Stadtstaaten Hamburg und Berlin wurde die Befragung auf Ebene der Stadtbezirke durchgeführt, sodass insgesamt 689 befragte Kommunen berücksichtigt wurden.

Aus Kommunen mit weniger Einwohnern wurde eine Zufallsstichprobe gezogen:

- Aus der Klasse der Kommunen mit 10.000 bis 20.000 Einwohnern wurden 200 von insgesamt 882 Kommunen befragt.

- Bei den Kommunen mit weniger als 10.000 Einwohnern wurden ebenfalls 200 von insgesamt 9.871 Kommunen ausgewählt.

Aus diesen drei Gruppen ergab sich eine Bruttostichprobe von 1.076 Kommunen, die alle Bundesländer umfasste. Als Adressaten der ersten Befragung wurden die Planungsamtsleiter festgelegt. Der Fragebogen der ersten Phase ist als Anhang 1 beigefügt.

\section{Rücklauf in Phase I}

Aus der ersten Befragungsphase wurden Fragebögen von 359 Kommunen ausgewertet, dies entspricht einem Anteil von $33 \%$ an der Bruttostichprobe. Die Kommunen verteilen sich über das ganze Bundesgebiet (Abbildung 2).

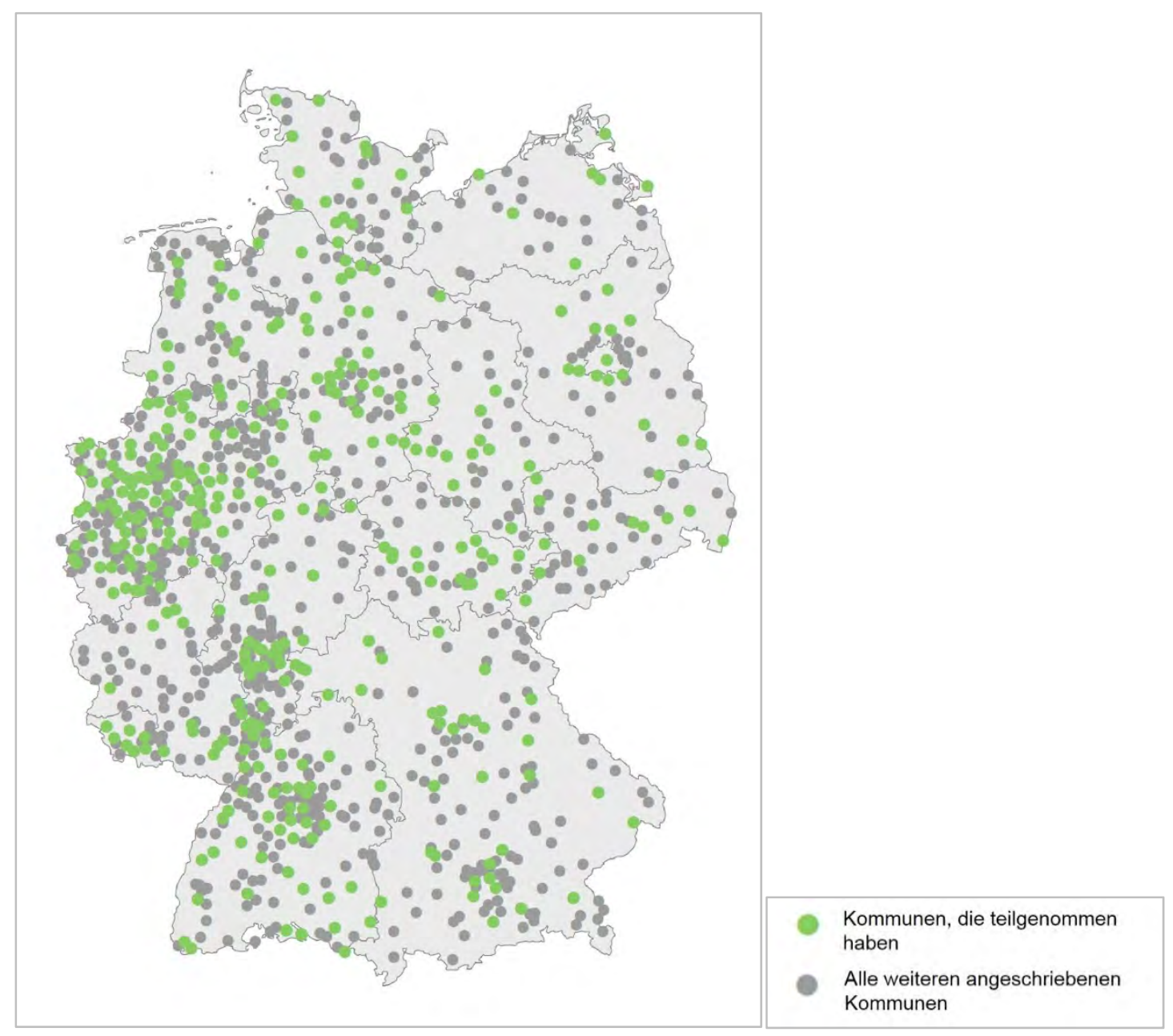

Abbildung 2: $\quad$ Verteilung über das Bundesgebiet: Stichprobe $(n=1.076)$ und Teilnehmer an der Befragung $(n=359)$ 
Das Bundesland Hamburg ist nicht in der Erhebung vertreten. Aus dem Bundesland Bremen hat sich nur die Stadt Bremerhaven beteiligt. Gemessen an der Anzahl der Kommunen im Rücklauf der Erhebung sind die Bundesländer Bayern, Mecklenburg-Vorpommern, Rheinland-Pfalz und Sachsen in der Erhebung unterrepräsentiert (Abbildung 3). Die Verteilung der Kommunen auf die Einwohnerklassen ist in Abbildung 4 wiedergeben. Die Einteilung der Ortsgrößenklassen beruht auf den Stadt- und Gemeindetypen der Raumabgrenzungen des Bundesinstituts für Bau-, Stadtund Raumforschung (BBSR).

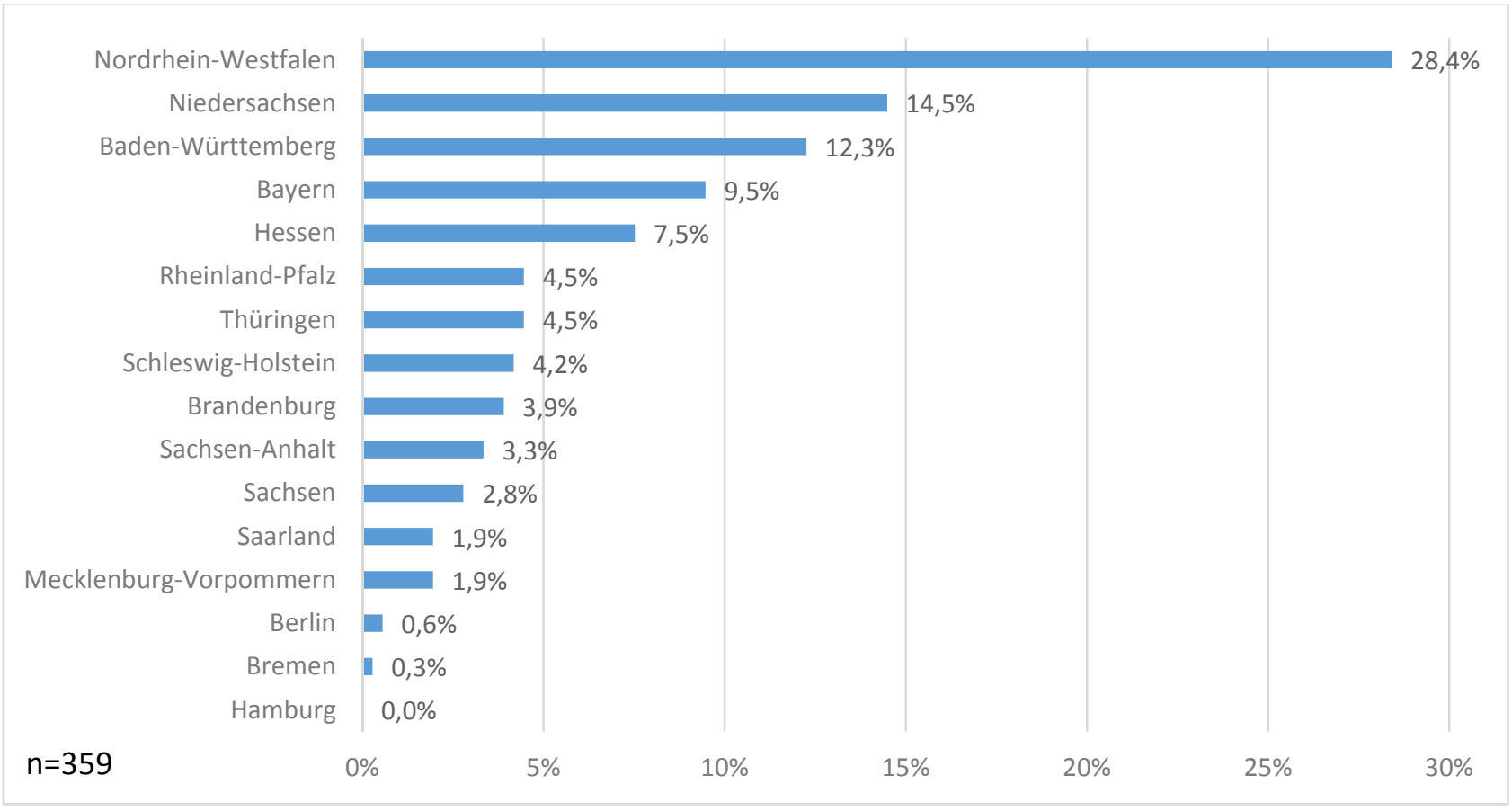

Abbildung 3: Verteilung der teilnehmenden Kommunen nach Bundesländern in \% $(n=359)$

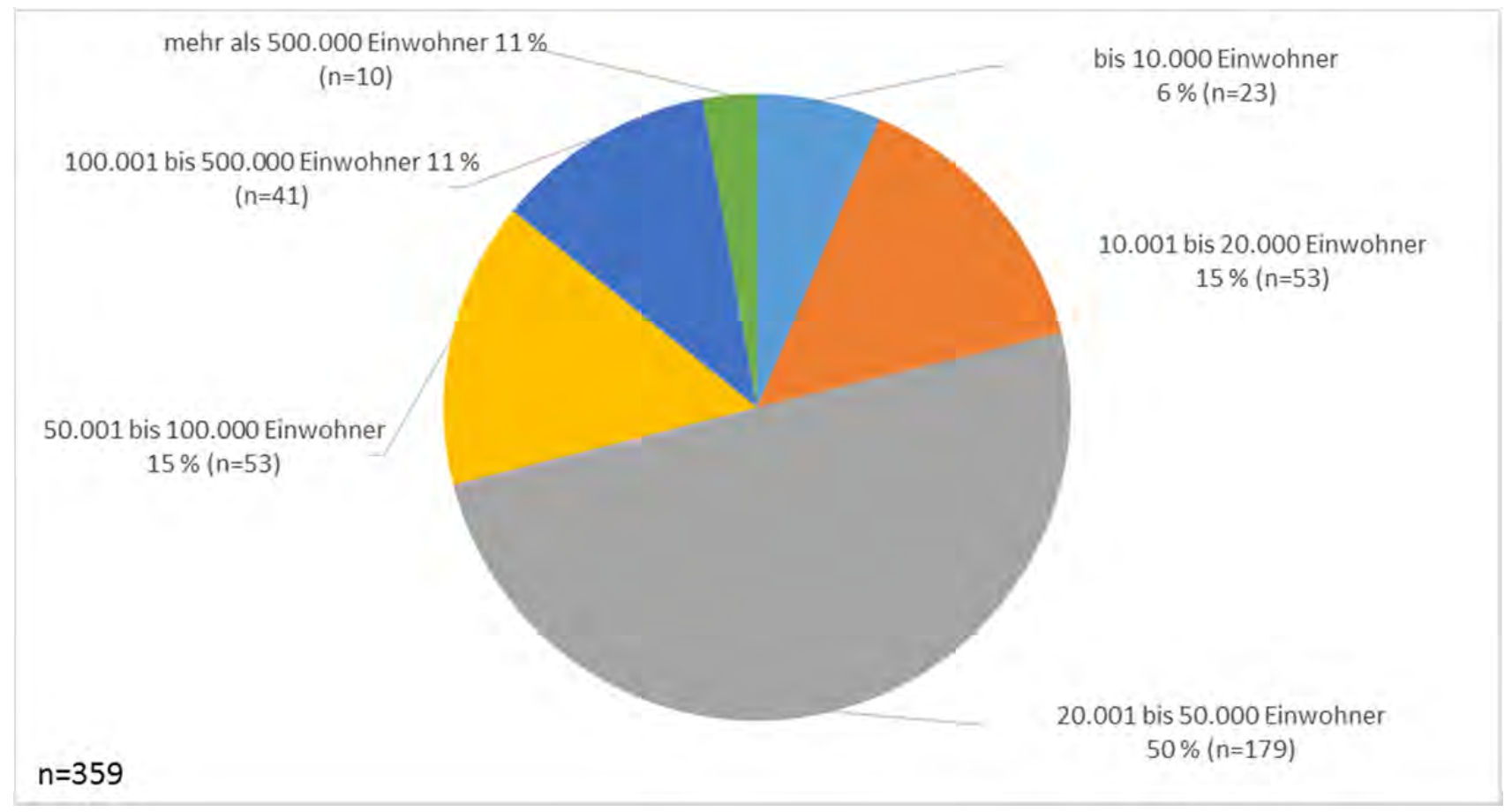

Abbildung 4: $\quad$ Verteilung der teilnehmenden Kommunen auf die Ortsgrößenklassen $(n=359)$ 
Auf Ebene der kommunalen Größenklassen zeigt sich, dass Städte und Gemeinden bis 20.000 Einwohner weniger häufig teilgenommen haben als die größeren Städte. Aufgrund der geringen Beteiligung der kleineren Kommunen unter 20.000 Einwohnern an der Erhebung und der darüber hinaus nicht-proportionalen Verteilung auf die Bundesländer lassen sich für die Größenklassen keine spezifischen Aussagen treffen (Abbildung 5).

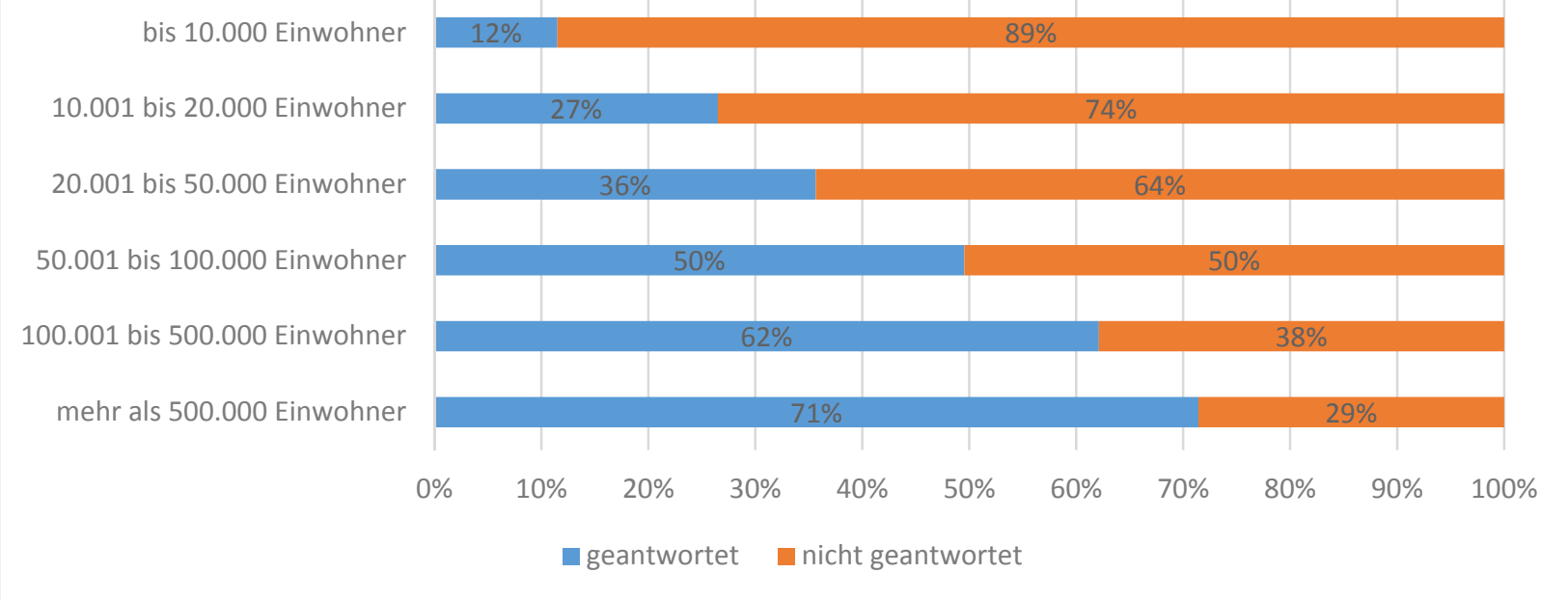

Abbildung 5: Beteiligung an der Kommunalbefragung nach Kommunengröße $(n=1.076)$

Grundsätzlich ist der Rücklauf jedoch zufriedenstellend und erlaubt allgemeine Rückschlüsse über die Verbreitung, Nutzung und Gestaltung von Fahrradstraßen und geöffneten Einbahnstraßen.

\section{Phase II der Befragung: Fahrradstraßen}

Da für Fahrradstraßen bislang keine umfassenden Untersuchungen vorliegen, wurde jede in Phase I gemeldete Fahrradstraße in die zweite Phase der Erhebung einbezogen. Die Kommunen wurden gebeten, für jede Fahrradstraße einen eigenen Erhebungsbogen auszufüllen. Falls dies von den Kommunen nicht geleistet werden konnte, sollten zumindest für die aus kommunaler Sicht „konfliktträchtigen“ bzw. die ihrer Meinung nach interessanten Fahrradstraßen geantwortet werden.

Ausgewählte Kommunen, von denen bekannt ist, dass sie Fahrradstraßen ausgewiesen haben, die aber nicht an der Befragung teilgenommen haben, wurden nochmal gesondert kontaktiert und um Beteiligung gebeten.

Zwei Kommunen ${ }^{5}$ haben im Verhältnis zur restlichen Stichprobe außergewöhnlich viele Fahrradstraßen ausgewiesen. Daher bestand die Gefahr, dass diese beiden Städte das Ergebnis beeinflussen könnten. Eine mögliche Verzerrung des Ergebnisses wurde für den Mehrwert, eine möglichst hohe Anzahl von Fahrradstraßen in der Befragung zu haben, in Kauf genommen und wurde bei der Interpretation der Ergebnisse berücksichtigt.

Der Erhebungsbogen ist als Anhang beigefügt (Anhang 2).

\section{Rücklauf bei den Fahrradstraßen}

Von den in Phase 1 genannten 426 Fahrradstraßen wurden für 179 Fahrradstraßen Angaben in der Phase 2 getätigt, was einer Rücklaufquote von $41 \%$ entspricht. Der Rücklauf bietet eine gute Grundlage für verallgemeinerbare Aussagen über Fahrradstraßen.

\footnotetext{
${ }^{5}$ Es handelt sich um die Städte München mit 47 und Braunschweig mit 40 Fahrradstraßen
} 
Die Verteilung der an Phase II der Befragung teilnehmenden Kommunen nach Bundesländern zeigt, dass NRW deutlich stärker vertreten ist, als andere Bundesländer (Abbildung 6). Die Bundesländer Berlin, Bremen, Hamburg, Rheinland-Pfalz und Sachsen sind nicht in Phase II Fahrradstraßen vertreten.

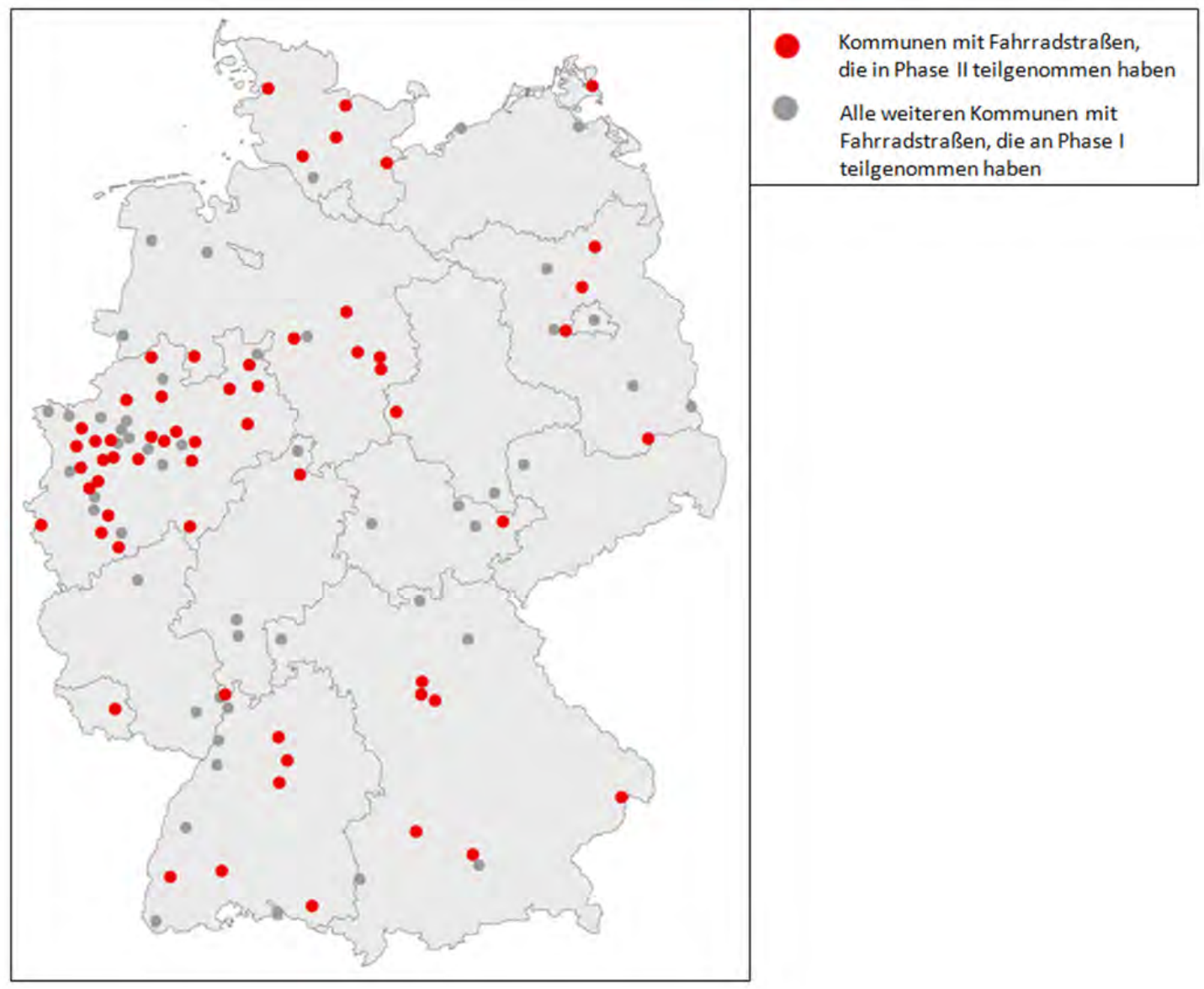

Abbildung 6: Verteilung der Stichprobe Phase II - Kommunen mit Fahrradstraßen $(\mathrm{n}=110)$ und an Phase II teilnehmende Kommunen $(n=61)$

\section{Phase II der Befragung: Geöffnete Einbahnstraßen}

In der zweiten Phase der Befragung wurden nur die geöffneten Einbahnstraßen berücksichtigt, die von den Kommunen als problematisch/unfallträchtig bewertet wurden. Dadurch sollte die Zahl der zu untersuchenden geöffneten Einbahnstraßen überschaubar gehalten werden, weil bereits Untersuchungen und Aussagen zu diesem Themenbereich existieren und hier nur geprüft werden sollte, ob neu aufgetretene Probleme ergänzenden Untersuchungsbedarf erfordern. Die Kommunen wurden gebeten, für diese Straßen jeweils einen eigenen Fragebogen auszufüllen. Der Fragebogen ist als Anhang beigefügt (Anhang 3).

Da die meisten Kommunen ihre geöffneten Einbahnstraßen als unproblematisch bezeichneten oder dazu keine Angaben machten, wurden insgesamt „nur“ 25 geöffnete Einbahnstraßen aus 15 Kommunen in Phase II berücksichtigt. Von den 25 geöffneten Einbahnstraßen wurden für 17 Straßen Angaben gemacht.

Aufgrund der geringen Fallzahlen von problematischen geöffneten Einbahnstraßen in Phase II wurde ergänzend in der Unfallanalyse eine zusätzliche flächendeckende Erhebung aller Unfälle in Einbahnstraßen in einem begrenzten Bereich einer Kommune (Innenstadt von Köln, siehe Kapitel 4.3) durchgeführt. 


\section{$2.3 \quad$ Unfallanalyse}

Im Anschluss an die Kommunalbefragung wurde in den bei der Umfrage erfassten Fahrradstraßen und den als problematisch / unfallauffällig gemeldeten, geöffneten Einbahnstraßen eine Analyse des Unfallgeschehens durchgeführt. Diese hatte zum Ziel, einen quantitativen Eindruck über das Unfallgeschehen insgesamt zu bekommen und erste Hinweise auf Sicherheitsdefizite für den Radverkehr zu identifizieren.

Die Unfälle wurden unabhängig von der Verkehrsbeteiligung für die Jahre 2008 - 2012 analysiert, allerdings wurden nur die Unfälle mit Personenschaden in die Auswertung mit einbezogen. Für Fahrradstraßen und geöffnete Einbahnstraßen zusammen wurden aus 68 Kommunen in 235 Straßen 1.024 Unfälle in die makroskopische Auswertung einbezogen.

In der mikroskopischen Unfallanalyse wurde vor allem untersucht, ob typische Unfallsituationen in den beiden Infrastrukturelementen identifiziert werden können und ob infrastrukturell bedingte Verkehrssicherheitsprobleme vorliegen. Dabei werden die einzelnen Unfälle nicht nur mit den anderen Unfällen in den jeweiligen Straßen verglichen, sondern auch mit anderen Einzelunfällen, die die gleiche Charakteristik aufweisen.

In den Ergebnissen werden die Daten aus mikroskopischer und makroskopischer Analyse zusammengeführt.

\subsubsection{Unfallanalyse in Fahrradstraßen: Datengrundlage und Datenaufbereitung}

Für alle Kommunen, die sich an der zweiten Phase der Kommunalbefragung beteiligt haben, wurden in den genannten Fahrradstraßen alle Unfälle mit Personenschaden $(U(P))$ aus dem Zeitraum 2008 bis 2012 angefordert.

Die UDV stellte für die beteiligten Kommunen aus folgenden Bundesländern EUSKA-Datensätze zur Verfügung: Baden-Württemberg, Berlin, Hessen, Sachsen, Sachsen-Anhalt und Thüringen. Zusätzlich wurden in Nordrhein-Westfalen für einzelne Städte Unfälle im genannten Zeitraum im EUSKA-Format zur Verfügung gestellt.

Für die Kommunen in den anderen Bundesländern sowie den übrigen Kommunen in NordrheinWestfalen wurden die Unfalldaten in der Regel über die Landespolizeidienststellen angefordert. Die in unterschiedlichen Formaten gelieferten Daten wurden harmonisiert. In Nordrhein-Westfalen wurde die Geocodierung auf Basis eines Straßenschlüssels und der Angabe der Hausnummer nachcodiert. ${ }^{6}$

Die Landespolizeidienststellen in den drei Bundesländern Mecklenburg-Vorpommern, RheinlandPfalz und Saarland gaben die Auskunft, dass für die angeforderten Straßen im Zeitraum zwischen 2008 und 2012 keine Unfälle polizeilich registriert worden sind ${ }^{7}$. Das Land Brandenburg konnte nur für einen abweichenden Vierjahreszeitraum von 2010 bis 2013 die Unfalldaten elektronisch zur Verfügung stellen; die Brandenburger Unfälle wurden daraufhin aus der Unfallanalyse herausgenommen. ${ }^{8}$

6 Die Unfälle in zwei Fahrradstraßen und einer Einbahnstraße konnten nicht verortet werden und sind nicht in die Auswertungsdatenbank integriert worden.

7 In den Bundesländern Mecklenburg-Vorpommern, Rheinland-Pfalz und Saarland lagen in insgesamt vier Straßen in vier Kommunen keine polizeilich registrierten Unfälle vor.

820 Unfälle in vier Städten in Brandenburg wurden aufgrund des abweichenden Unfallzeitraumes nicht in die Auswertung mit einbezogen. 
Schließlich wurden alle gemeldeten Unfälle mit dem Einrichtungsjahr der Fahrradstraße bzw. der Öffnung der Einbahnstraße abgeglichen. 120 Unfälle sind vor dem Einrichtungsdatum polizeilich registriert worden, diese wurden ebenfalls aus der Analysedatenbank entfernt.

Die folgende Abbildung 7 zeigt die Stichprobe der Fahrradstraßen räumlich verortet:

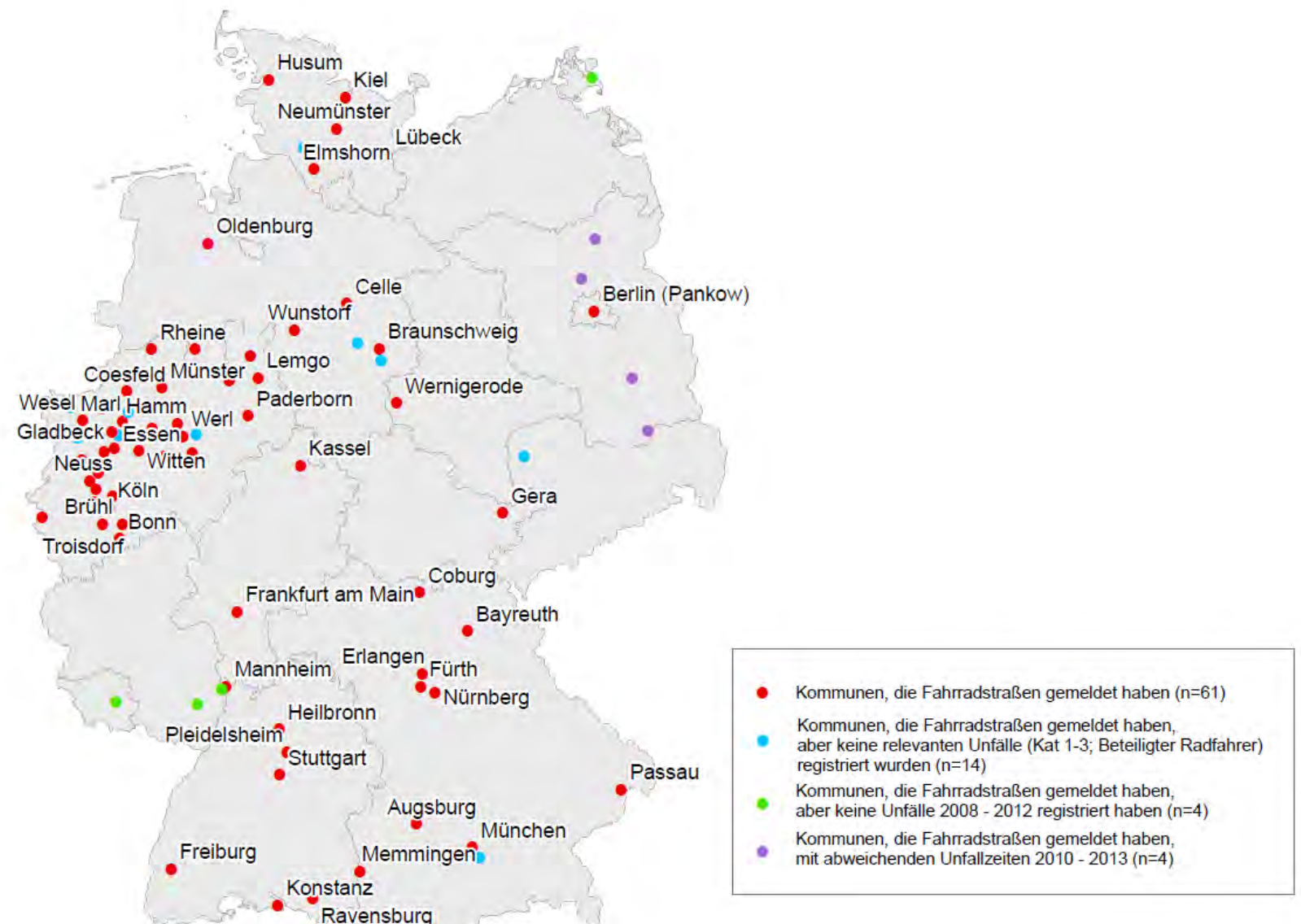

Abbildung 7: Kommunen, die Fahrradstraßen gemeldet haben

Im Rahmen der Mikroanalyse wurden alle beteiligten Kommunen gebeten, Unfallhergänge bzw. Unfalltexte zu den makroskopisch untersuchten Unfällen zu liefern.

Für 607 Unfälle wurden Unfallhergänge bzw. Unfalltexte zur Verfügung gestellt. Diese Unfälle wurden detailliert analysiert und daraufhin bewertet, ob die Unfälle

- im Zuge einer Fahrradstraße oder beim Ein- und Ausfahren des Radverkehrs aus den angrenzenden Netzbestandteilen geschehen sind und gleichzeitig

- ihre Ursache im Zusammenhang mit den baulichen und verkehrstechnischen Gegebenheiten haben können.

186 Unfälle wurden bei detaillierter Überprüfung des Unfallhergangs und des Unfallortes aufgrund dieser Bewertungskriterien als relevant eingestuft.

421 Unfälle wurden dagegen als nicht relevant eingestuft. Dazu gehören unter anderem Unfälle,

- an denen keine Radfahrer beteiligt waren, 
- an den Anfangs-/Endknotenpunkten, die in Zusammenhang mit der kreuzenden Straße stehen, aber nicht mit der Fahrradstraße (mit Abstand häufigster Ausschlussgrund),

- auf angrenzenden Privatgrundstücken, Parkplätzen, Hinterhöfen, etc.,

- mit alkoholisierten Personen, die grundsätzlich nicht vertieft untersucht wurden (auch bei mehreren Unfallbeteiligten),

- die Auffahrunfälle waren (Z.B. Pkw-Pkw, Grund: Unachtsamkeit) oder Unfälle beim EinIAusfahren aus dem Grundstück (weil nicht geblinkt, etc.) oder Unfälle mit Pkw in Gegenverkehr (bei ausreichend breiten Fahrbahnen),

- weitere Alleinunfälle: Skateboardfahrer fährt auf Fahrbahn ohne auf Verkehr zu achten, Radfahrer fährt auf Gehweg auf und stürzt an Bordsteinkante, etc.

In der mikroskopischen Unfalluntersuchung wurden nur noch die relevanten Unfälle mit Radverkehrsbeteiligung (186 Unfälle) untersucht. Tabelle 1 gibt noch einmal einen Überblick über die Anzahl der untersuchten Kommunen und Fahrradstraßen sowie über die Anzahl der untersuchten Unfälle in diesen Fahrradstraßen in den einzelnen Analyseschritten.

Tabelle 1: $\quad$ Übersicht der untersuchten Fahrradstraßen und Unfälle in der Mikroanalyse

\begin{tabular}{|c|c|c|c|c|c|c|}
\hline & $\begin{array}{l}\text { Anzahl der } \\
\text { Kommunen } \\
\text { mit Fahrrad- } \\
\text { straßen mit } \\
\text { Unfällen }\end{array}$ & $\begin{array}{c}\text { Anteile an } \\
\text { allen } \\
\text { Kommunen } \\
\text { mit Fahrrad- } \\
\text { straßen mit } \\
\text { Unfällen }\end{array}$ & $\begin{array}{l}\text { Anzahl der } \\
\text { Fahrrad- } \\
\text { straßen mit } \\
\text { Unfällen }\end{array}$ & $\begin{array}{c}\text { Anteile an } \\
\text { allen Fahrrad- } \\
\text { straßen mit } \\
\text { Unfällen }\end{array}$ & $\begin{array}{l}\text { Anzahl der } \\
\text { Unfälle }\end{array}$ & $\begin{array}{c}\text { Anteile an } \\
\text { allen Unfällen }\end{array}$ \\
\hline $\begin{array}{l}\text { Makroanalyse: } \\
\text { alle Unfälle }\end{array}$ & 61 & $100 \%$ & 223 & $100 \%$ & 863 & $100 \%$ \\
\hline $\begin{array}{l}\text { Makroanalyse: } \\
\text { ohne Radverkehrsunfälle }\end{array}$ & 5 & $8 \%$ & 42 & $19 \%$ & 284 & $33 \%$ \\
\hline $\begin{array}{l}\text { Makroanalyse: } \\
\text { mit Radverkehrsunfällen }\end{array}$ & 56 & $92 \%$ & 181 & $81 \%$ & 579 & $67 \%$ \\
\hline $\begin{array}{l}\text { Mikroanalyse: } \\
\text { alle Unfälle }\end{array}$ & 47 & $100 \%$ & 177 & $100 \%$ & 607 & $100 \%$ \\
\hline $\begin{array}{l}\text { Mikroanalyse: } \\
\text { Unfälle ohne Radverkehrsbeteiligung und nicht } \\
\text { relevante Radverkehrsunfälle }\end{array}$ & 15 & $32 \%$ & 102 & $58 \%$ & 421 & $69 \%$ \\
\hline $\begin{array}{l}\text { Mikroanalyse: } \\
\text { relevante Radverkehrsunfälle }\end{array}$ & 32 & $68 \%$ & 75 & $42 \%$ & 186 & $31 \%$ \\
\hline $\begin{array}{r}\text { Mikroanalyse: } \\
\text { nur relevante Radverkehrsunfälle am } \\
\text { Zwischenknotenpunkt }\end{array}$ & 23 & & 35 & & 65 & $35 \%$ \\
\hline $\begin{array}{r}\text { Mikroanalyse: } \\
\text { nur relevante Radverkehrsunfälle auf Strecke }\end{array}$ & 16 & & 35 & & 63 & $34 \%$ \\
\hline $\begin{array}{r}\text { Mikroanalyse: } \\
\text { nur relevante Radverkehrsunfalle an anderen Orten }\end{array}$ & 19 & & 34 & & 58 & $31 \%$ \\
\hline
\end{tabular}

Die mikroskopischen Unfalldaten, die zur Verfügung gestellt wurden, stehen in Hinblick auf ihre Relevanz in Bezug auf die Infrastruktur Fahrradstraße in folgendem Verhältnis (Abbildung 8): 


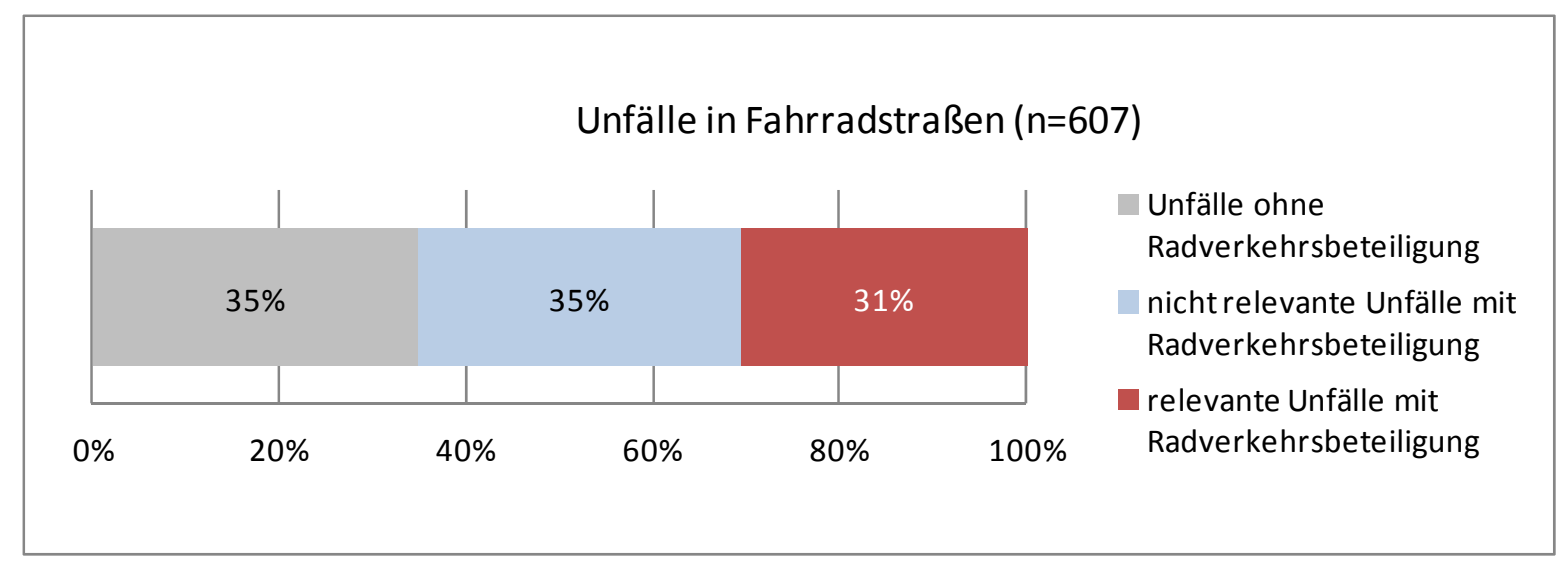

Abbildung 8: Verhältnis relevanter und nicht relevanter Unfälle in Fahrradstraßen für die mikroskopische Unfalluntersuchung (U(P), 2008-2012)

Dabei wird deutlich, dass nur ein Drittel der polizeilich gemeldeten Radverkehrsunfälle in Fahrradstraßen für die vorliegende Untersuchung relevante Unfälle sind, d.h. Unfälle mit Radverkehrsbeteiligung, die in räumlichen Zusammenhang mit der Fahrradstraße stehen und ihre Ursache im Zusammenhang mit den baulichen und verkehrstechnischen Gegebenheiten haben können. Außerdem findet nur in weniger als der Hälfte der Fahrradstraßen (42\%) ${ }^{9}$ mindestens ein relevanter Unfall mit Radverkehrsbeteiligung im untersuchten Fünfjahreszeitraum statt.

Die Ausführungen in Kapitel 3.3 zeigen die Ergebnisse der Unfallanalyse nach folgendem Muster:

- Zunächst werden die Ergebnisse der makroskopischen Untersuchung verdeutlicht: Das Gesamtunfallgeschehen mit Personenschaden in den Fahrradstraßen der Stichprobe unabhängig von der Verkehrsbeteiligung wird dem Unfallgeschehen mit Personenschäden in Fahrradstraßen, an denen Radfahrer beteiligt waren ${ }^{10}$, gegenübergestellt. Verglichen werden diese Daten mit allen innerörtlichen bundesweiten Unfällen mit Personenschaden unabhängig von der Verkehrsbeteiligung und mit allen innerörtlichen bundesweiten Unfällen mit Radverkehrsbeteiligung aus den Jahren 2008 bis $2012 .^{11}$

Zusätzlich zu den bundesweiten innerörtlichen Unfällen mit Personenschaden wurden als weitere Vergleichsbasis Unfalldaten aus geeigneten Nebenstraßen als kleinere Stichprobe ${ }^{12}$ sowie Unfalldaten aus innerörtlichen Tempo 30-Zonen der Bundesländer Baden-Württemberg, Hessen, Sachsen und Sachsen-Anhalt als größere Stichprobe ${ }^{13}$ herangezogen. Für die Unfallkategorie, den Unfalltyp, die Unfallart und die Unfallcharakteristik wurden diese Daten als Vergleichsbasis ausgewertet. Hierbei wurden jedoch keine wesentlichen Unterschiede zu den bundesweiten innerörtlichen Unfällen festgestellt. Die Ergebnisse zu den Nebenstraßen werden in den Diagrammen zu Unfallschwere und Unfalltyp dargestellt, in den folgenden Diagrammen wurde auf eine gesonderte Darstellung dieser Werte verzichtet.

- An die Auswertungen der makroskopischen Unfallanalyse schließen sich jeweils die der mikroskopischen an. Hier werden nur die Ergebnisse zu den Unfällen mit

9 Siehe Tabelle 1

10 stark farbige Balken 2 und 3 der Grafiken

11 Quelle: Statistisches Bundesamt, Wiesbaden 2014, transparent farbige Balken 1 und 4 der Grafiken

$12 n=101$

$13 \mathrm{n}=24.547$ 
Radverkehrsbeteiligung aufgeführt: Die Radverkehrsunfälle in Fahrradstraßen (Makroanalyse, Balken 2) werden den relevanten Radverkehrsunfällen in Fahrradstraßen (Mikroanalyse, Balken 3) gegenübergestellt. Erneut werden diese Daten mit allen innerörtlichen bundesweiten Unfällen mit Radverkehrsbeteiligung verglichen.

- Die Mikroanalyse hat ergeben, dass sich die Unfälle nach Unfallort in ihrer Charakteristik unterscheiden: Streckenunfälle weisen eine typische Charakteristik auf, die sich von Zwischenknotenpunktunfällen stark unterscheidet.

Die Unfälle an den Anfangs- und Endknotenpunkten jedoch waren aufgrund der unterschiedlichen örtlichen Gegebenheiten so heterogen, dass eine zusammenfassende Untersuchung hier nicht sinnvoll erschien, da dies einer Einzelfallbetrachtung entsprochen hätte.

Abschließend wurden in einem dritten Auswertungsschritt die mikroskopischen Ergebnisse der relevanten Radverkehrsunfälle in Fahrradstraßen im Zwischenknoten denen auf der Strecke gegenübergestellt, um die Unterschiede herausheben zu können.

In den untersuchten Fahrradstraßen fanden ca. ein Drittel der Unfälle an Zwischenknotenpunkten und ein Drittel auf der Strecke statt. Die restlichen Unfälle fanden an den Anfangs- und Endknotenpunkten statt sowie an sonstigen Orten wie z.B. an Bushaltestellen oder Fußgängerüberwegen. Abbildung 9 verdeutlicht den Unfallort der relevanten Radverkehrsunfälle in den untersuchten Fahrradstraßen.

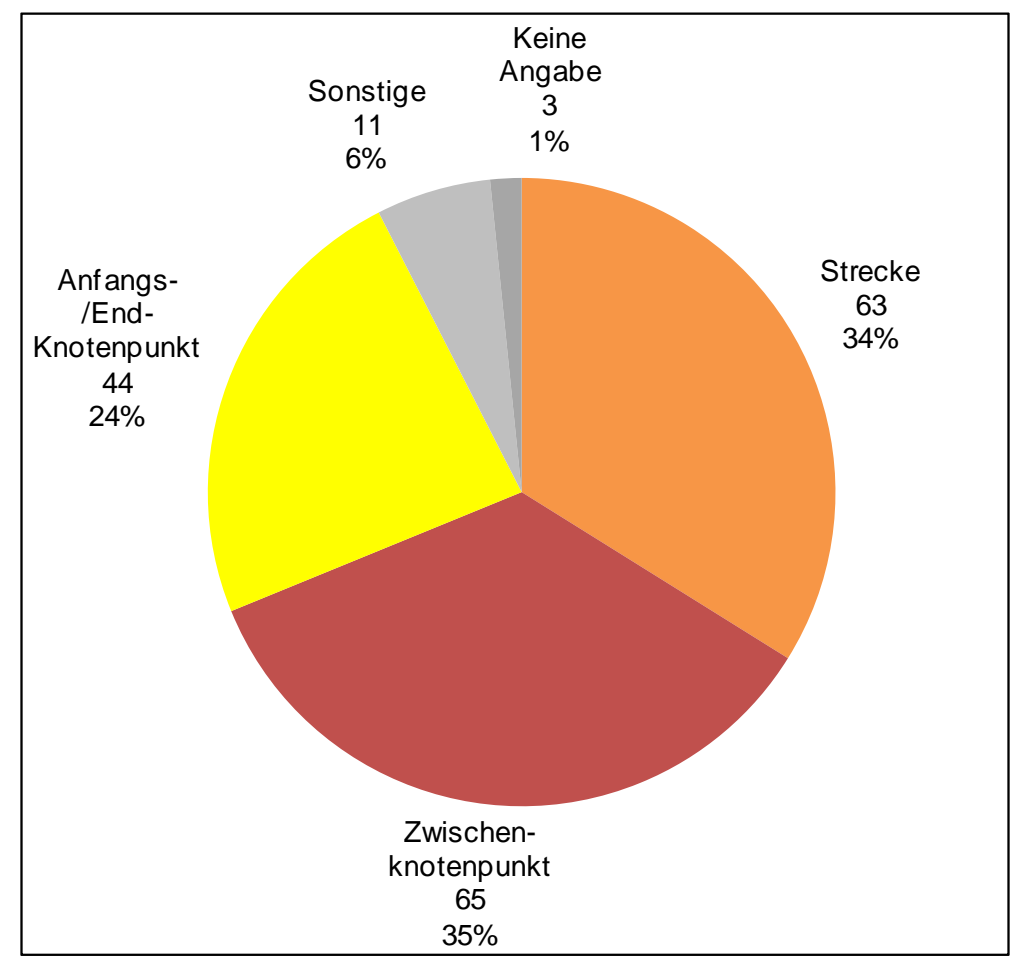

Abbildung 9: $\quad$ Unfallort der relevanten Radverkehrsunfälle in Fahrradstraßen (Unfälle mit Personenschaden $(U(P)$, 2008-2012) $(n=186)$

In den Kapiteln zu den Ergebnissen der Unfallanalyse werden die Unfalldaten zu Unfallkategorie, Unfalltyp, Unfallart, Charakteristik der Unfallstelle, Unfallbeteiligung, Unfallursachen und Unfallkonstellationen ausgewertet und die Ergebnisse dargestellt.

Darüber hinaus wurde die Unfallstunde von Unfällen mit Radverkehrsbeteiligung in Fahrradstraßen mit der Unfallstunde der bundesweiten innerörtlichen Radverkehrsunfälle verglichen. Da sich die Unfallstundendaten sehr stark decken, wurde auf die Darstellung verzichtet. Die Angaben in den 
Unfalldaten zu den Lichtverhältnissen und dem Fahrbahnzustand wurden ebenso ausgewertet, die Ergebnisse sind unauffällig. Die Anzahl der Nennungen zu den Besonderheiten der Unfallstelle ist zu gering, um statistisch ausgewertet zu können. ${ }^{14}$

Ebenso wurden die soziodemografischen Variablen Alter und Geschlecht ausgewertet. Es wurde überprüft, ob Pkw-Fahrer als Unfallgegner auffällig häufig einer bestimmten Altersklasse zuzuordnen waren; die Ergebnisse sind unauffällig. Das Geschlecht der beteiligten Pkw- und Radfahrer wurde überprüft; die Ergebnisse sind ebenfalls unauffällig. Auch diese Ergebnisse werden deshalb nicht dargestellt.

\subsubsection{Unfallanalyse in geöffneten Einbahnstraßen: Datengrundlage und Datenaufbereitung}

In der Kommunalbefragung wurden die Angeschriebenen gefragt, ob es in ihrer Kommune geöffnete Einbahnstraßen gibt und ob diese ihrer Einschätzung nach unfallauffällig sind. Damit wurde die Verbreitung von geöffneten Einbahnstraßen erstmalig flächendeckend erhoben. Der Rücklauf zeigt auf, dass geöffnete Einbahnstraßen ein weit verbreitetes Infrastrukturelement sind und dass diese nur selten als problematisch wahrgenommen werden (siehe Kap. 4.2).

Für 19 als unfallauffällig gemeldete Einbahnstraßen wurden Unfalldaten für die makroskopische Unfalluntersuchung zur Verfügung gestellt. In diesen problematischen Einbahnstraßen sind 161 Unfälle im Zeitraum zwischen 2008 und 2012 polizeilich registriert worden, davon 99 mit Radverkehrsbeteiligung. Abbildung 10 führt die Kommunen auf, die in der Kommunalbefragung gemeldet haben, es gäbe in ihrer Kommune unfallauffällige geöffnete Einbahnstraßen.

$14 \mathrm{n}=24, \mathrm{k} . \mathrm{A} .=555$ 


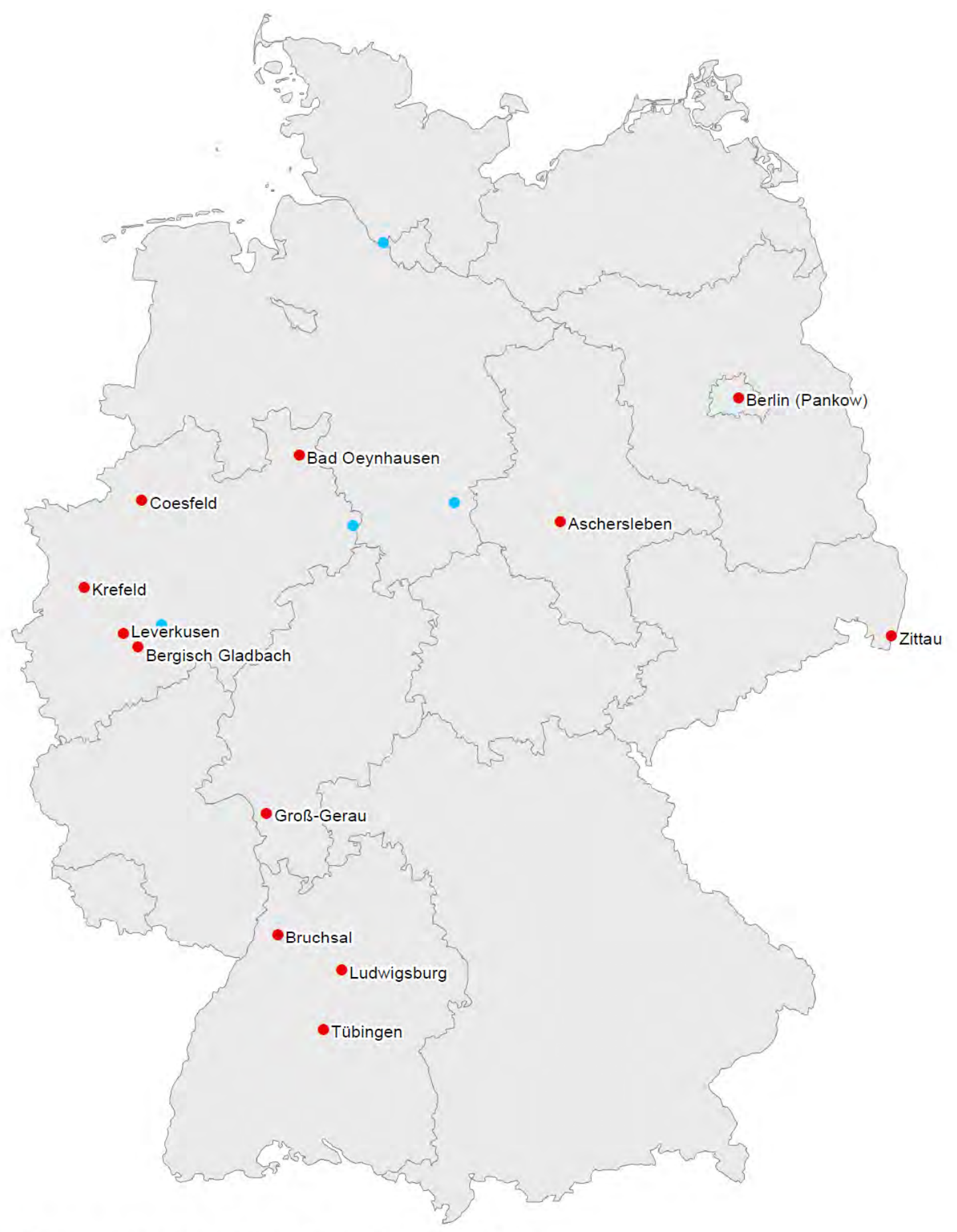

- Kommunen, die problematische Einbahnstraßen

gemeldet haben $(n=12)$

Kommunen, die problematische Einbahnstraßen gemeldet

- haben, für die aber keine relevanten Unfälle

(Kat 1-3; Beteiligter Radfahrer) festgestellt wurden $(n=4)$

Abbildung 10: Kommunen, die problematische, geöffnete Einbahnstraßen gemeldet haben

Insgesamt war die Fallzahl der Stichprobe bereits in der Phase der makroskopischen Unfallanalyse sehr gering, so dass sich nur eingeschränkt repräsentative Schlussfolgerungen ziehen ließen und auch ein Vergleich mit der Datenbasis des Statistischen Bundesamtes zwar sinnvoll, aber nur eingeschränkt repräsentativ war. 
Die makroskopische Unfallauswertung erlaubt zudem keine Rückschlüsse darauf, ob die Unfälle mit Radfahrerbeteiligung Unfälle mit Radfahrern waren, die entgegen der Einbahnstraßenrichtung gefahren sind. Diese Information konnte nur die Auswertung der Unfallhergänge im Rahmen der Mikroanalyse liefern.

Im Rahmen der Mikroanalyse wurden die betreffenden Kommunen gebeten, Unfallhergänge bzw. Unfalltexte zu den makroskopisch untersuchten Unfällen zu liefern. ${ }^{15}$

Für 152 Unfälle wurden Unfallhergänge bzw. Unfalltexte zur Verfügung gestellt. Diese Unfälle wurden detailliert analysiert und daraufhin bewertet, ob der Radverkehr gegen die allgemeine Fahrtrichtung unterwegs gewesen ist. 54 Unfälle wurden im Hinblick auf dieses Bewertungskriterium als relevant und 98 als nicht relevant bewertet.

Tabelle 2 gibt einen Überblick über die Anzahl der untersuchten Kommunen und geöffneten Einbahnstraßen sowie über die Anzahl der untersuchten Unfälle in diesen geöffneten Einbahnstraßen in den einzelnen Analyseschritten.

Tabelle 2: $\quad$ Übersicht der untersuchten geöffneten Einbahnstraßen und Unfälle in der Makro- und Mikroanalyse

\begin{tabular}{|c|c|c|c|c|c|c|}
\hline & $\begin{array}{c}\text { Anzahl der } \\
\text { Kommunen } \\
\text { mit Einbahn- } \\
\text { straßen mit } \\
\text { Unfällen }\end{array}$ & $\begin{array}{c}\text { Anteile an } \\
\text { allen } \\
\text { Kommunen } \\
\text { mit Einbahn- } \\
\text { straßen mit } \\
\text { Unfällen }\end{array}$ & $\begin{array}{l}\text { Anzahl der } \\
\text { Einbahn- } \\
\text { straßen mit } \\
\text { Unfällen }\end{array}$ & $\begin{array}{c}\text { Anteile an } \\
\text { allen Einbahn- } \\
\text { straßen mit } \\
\text { Unfällen }\end{array}$ & $\begin{array}{l}\text { Anzahl der } \\
\text { Unfälle }\end{array}$ & $\begin{array}{c}\text { Anteile an } \\
\text { allen Unfällen }\end{array}$ \\
\hline $\begin{array}{l}\text { Makroanalyse: } \\
\text { alle Unfälle }\end{array}$ & 12 & $100 \%$ & 19 & $100 \%$ & 161 & $100 \%$ \\
\hline $\begin{array}{l}\text { Makroanalyse: } \\
\text { ohne Radverkehrsunfälle }\end{array}$ & 0 & $0 \%$ & 1 & $5 \%$ & 62 & $39 \%$ \\
\hline $\begin{array}{l}\text { Makroanalyse: } \\
\text { mit Radverkehrsunfällen }\end{array}$ & 12 & $100 \%$ & 18 & $95 \%$ & 99 & $61 \%$ \\
\hline $\begin{array}{l}\text { Mikroanalyse: } \\
\text { alle Radverkehrsunfälle }\end{array}$ & 17 & $100 \%$ & 31 & $100 \%$ & 152 & $100 \%$ \\
\hline $\begin{array}{l}\text { Mikroanalyse: } \\
\text { nicht relevante Radverkehrsunfälle (Radfahrer fährt } \\
\text { nicht in Gegenrichtung) }\end{array}$ & 3 & $18 \%$ & 11 & $35 \%$ & 98 & $64 \%$ \\
\hline $\begin{array}{l}\text { Mikroanalyse: } \\
\text { relevante Radverkehrsunfälle (Radfahrer fährt in } \\
\text { Gegenrichtung) }\end{array}$ & 14 & $82 \%$ & 20 & $65 \%$ & 54 & $36 \%$ \\
\hline $\begin{array}{r}\text { Mikroanalyse: } \\
\text { nur relevante Radverkehrsunfälle am }\end{array}$ & 8 & & 10 & & 22 & $41 \%$ \\
\hline $\begin{array}{r}\text { Mikroanalyse: } \\
\text { nur relevante Radverkehrsunfälle auf Strecke }\end{array}$ & 9 & & 11 & & 15 & $28 \%$ \\
\hline nur relevante Radverkehrsunfälle an anderen Orten & 9 & & 9. & & 17 & $31 \%$ \\
\hline
\end{tabular}

Die mikroskopischen Unfalldaten, die zur Verfügung gestellt wurden, stehen in Hinblick auf ihre Relevanz in Bezug auf die Öffnung der Einbahnstraße für den Radverkehr in folgendem Verhältnis (Abbildung 11):

15 Während der detaillierten Analyse der Unfälle in der Mikroanalyse zeigte sich, dass einige Straßen, die als Fahrradstraßen gemeldet waren, „nur“ geöffnete Einbahnstraßen waren. Daher erhöhte sich die Stichprobe in der Mikroanalyse in Bezug auf die geöffneten Einbahnstraßen. Die Anzahl der Unfälle in diesen Straßen war jedoch gering (alle Unfälle $n=37$, Unfälle mit Radverkehrsbeteiligung $n=34$, relevante Unfälle mit Radverkehrsbeteiligung $n=27$ ). 


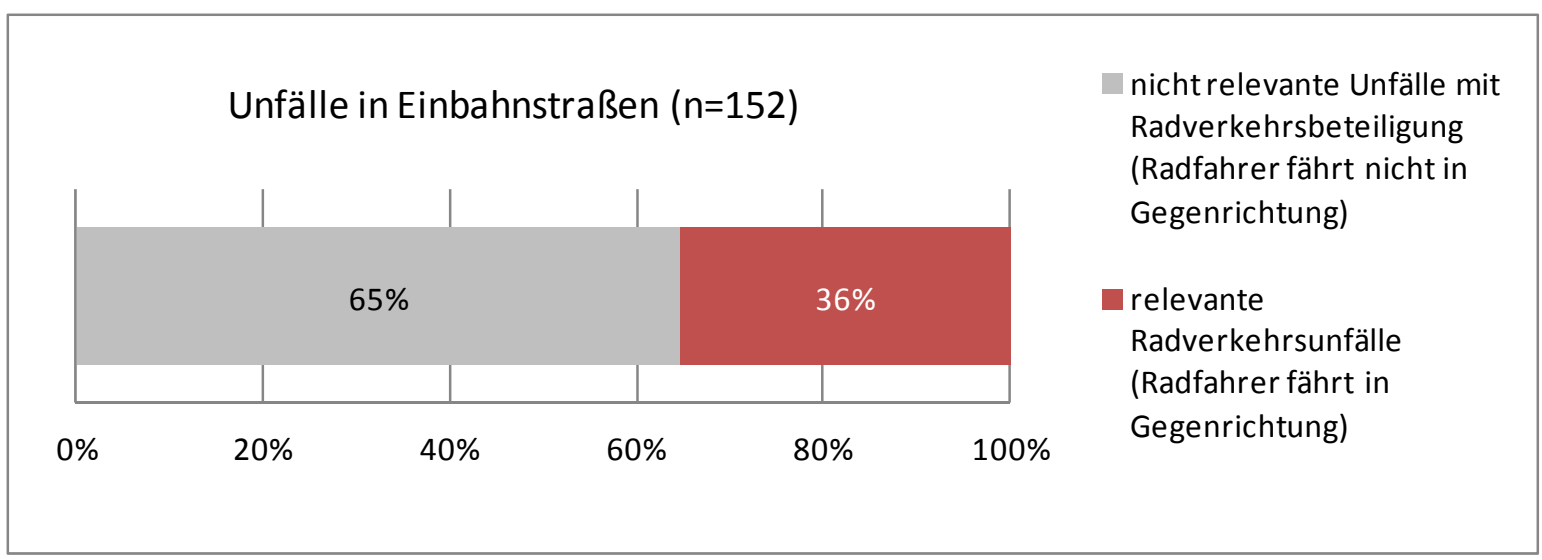

Abbildung 11: Verhältnis relevanter und nicht relevanter Unfälle in geöffneten Einbahnstraßen in der bundesweiten Untersuchung für die mikroskopische Unfalluntersuchung (Unfälle mit Personenschaden (U(P), 2008-2012)

Deutlich wird, dass nur bei ca. einem Drittel der Unfälle mit Radverkehrsbeteiligung in geöffneten Einbahnstraßen tatsächlich der Radverkehr in Gegenrichtung gefahren ist.

\section{Vergleichskollektiv Kölner Innenstadt}

Um die Frage zu überprüfen, inwieweit die in der bundesweiten Untersuchung analysierten geöffneten Einbahnstraßen repräsentativ sind, wurde ein weiteres Vergleichskollektiv herangezogen: Es wurden zusätzlich alle Unfälle in allen geöffneten Einbahnstraßen des Kölner Stadtbezirks 1 (Innenstadt und Deutz) untersucht. In den insgesamt 201 geöffneten Einbahnstraßen des Kölner Stadtbezirks 1 wurden 292 polizeilich registrierte Unfälle aus den Jahren 2008 bis 2012 untersucht. 63 Unfälle waren Unfälle, an den Radfahrer beteiligt waren, die in Gegenrichtung der Einbahnstraße fuhren, sogenannte „relevante“ Unfälle.

Tabelle 3 gibt einen Überblick über die Anzahl der geöffneten Einbahnstraßen in der Kölner Innenstadt sowie über die Anzahl der untersuchten Radverkehrsunfälle in diesen geöffneten Einbahnstraßen in den einzelnen Analyseschritten.

Tabelle 3: $\quad$ Übersicht der untersuchten geöffneten Einbahnstraßen und Radverkehrsunfälle in der Sonderuntersuchung für die Kölner Innenstadt (Radverkehrsunfälle mit Personenschaden (U(P), 2008-2012)

\begin{tabular}{|l|c|c|}
\hline & Anzahl & Anteile \\
\hline alle geöffneten Einbahnstraßen in der Kölner Innenstadt & 201 & $100 \%$ \\
\hline $\begin{array}{l}\text { geöffnete Einbahnstraßen in der Kölner Innenstadt ohne } \\
\text { Radverkehrsunfälle }\end{array}$ & 172 & $86 \%$ \\
\hline $\begin{array}{l}\text { geöffnete Einbahnstraßen in der Kölner Innenstadt mit relevanten } \\
\text { Radverkehrsunfällen (Radfahrer fährt in Gegenrichtung) }\end{array}$ & 29 & $14 \%$ \\
\hline $\begin{array}{l}\text { alle Radverkehrsunfälle in allen geöffneten Einbahnstraßen der Kölner } \\
\text { Innenstadt }\end{array}$ & 292 & $100 \%$ \\
\hline $\begin{array}{l}\text { nicht relevante Radverkehrsunfälle (Radfahrer fährt nicht in } \\
\text { Gegenrichtung) in den geöffneten Einbahnstraßen der Kölner Innenstadt }\end{array}$ & 229 & $78 \%$ \\
\hline $\begin{array}{l}\text { relevante Radverkehrsunfälle (Radfahrer fährt in Gegenrichtung) in den } \\
\text { geöffneten Einbahnstraßen der Kölner Innenstadt }\end{array}$ & 63 & $22 \%$ \\
\hline
\end{tabular}

Abbildung 12 zeigt für die Sonderuntersuchung zu den geöffneten Einbahnstraßen in der Kölner Innenstadt das Verhältnis der Anzahl von relevanten zu nicht relevanten Radverkehrsunfällen und das Verhältnis von Straßen mit relevanten und nicht relevanten Radverkehrsunfällen: 


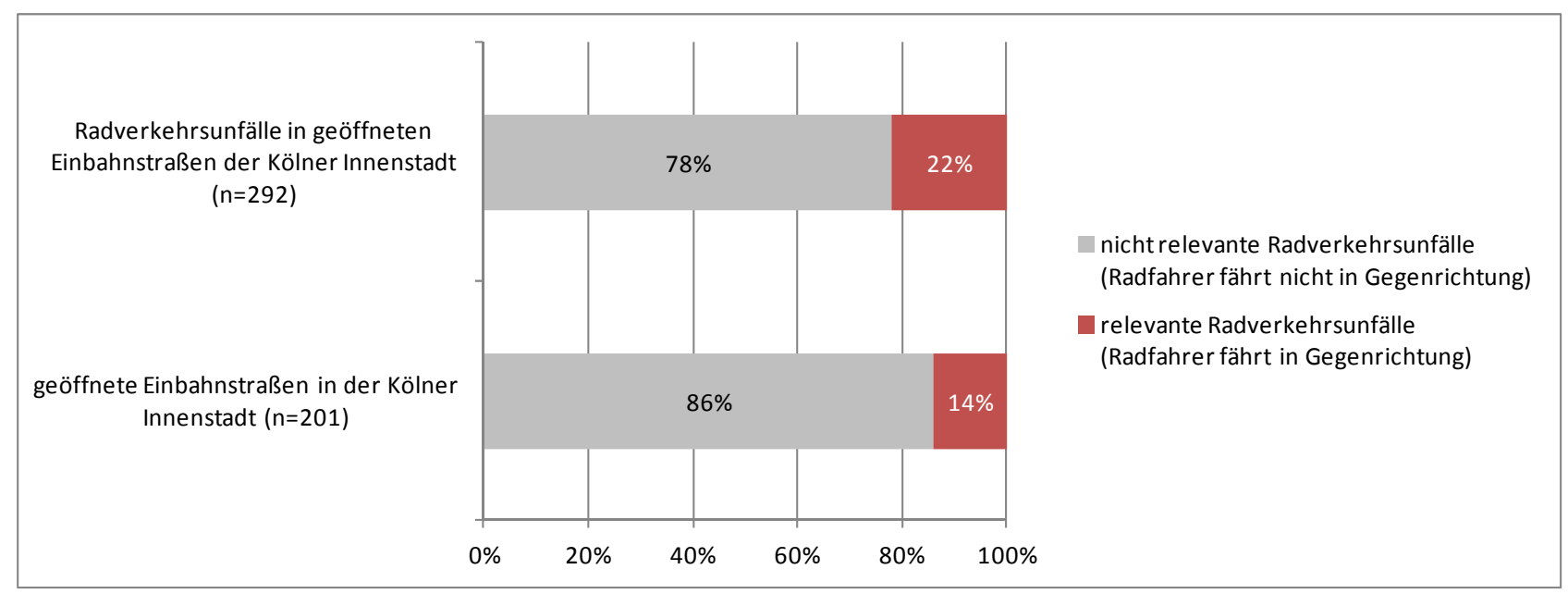

Abbildung 12: Verhältnis relevanter und nicht relevanter Unfälle in geöffneten Einbahnstraßen in der Sonderuntersuchung Kölner Innenstadt für die mikroskopische Unfalluntersuchung (Unfälle mit Personenschaden (U(P), 2008-2012)

In der Sonderuntersuchung wurden ausnahmslos alle geöffneten Einbahnstraßen in der Kölner Innenstadt untersucht: Nur wenige Straßen wiesen relevante Radverkehrsunfälle auf. Der Anteil von relevanten Radverkehrsunfällen zu nicht relevanten Radverkehrsunfällen beträgt ein Viertel. Damit konnten die Kernaussagen der bundesweiten Untersuchung zu den geöffneten Einbahnstraßen gestützt werden: Nur sehr wenige geöffneten Einbahnstraßen sind unfallauffällig und nur wenige Unfälle in geöffneten Einbahnstraßen passieren unter Beteiligung von Radfahrern, die in Gegenrichtung fahren.

Die Ausführungen in Kap. 4.3 zeigen die Ergebnisse der Unfallanalyse nach folgendem Darstellungsmuster:

- Zunächst werden die Ergebnisse der makroskopischen Auswertungen verdeutlicht: Die Unfälle der Stichprobe in den geöffneten Einbahnstraßen mit Personenschaden, an denen Radfahrer beteiligt waren, werden verglichen mit den Daten für alle innerörtlichen bundesweiten Unfälle mit Personenschaden und mit Radverkehrsbeteiligung aus den Jahren 2008 bis $2012 .^{16}$

- An die Auswertungen der makroskopischen Unfallanalyse schließen sich jeweils die der mikroskopischen an. Hier werden nur die Ergebnisse zu den relevanten Radverkehrsunfällen (Radfahrer fährt in Gegenrichtung) in geöffneten Einbahnstraßen dargestellt. ${ }^{17}$

- Zuletzt werden die Ergebnisse der relevanten Radverkehrsunfälle (Radfahrer fährt in Gegenrichtung) in den geöffneten Einbahnstraßen der Kölner Innenstadt aufgeführt. ${ }^{18}$

Die Mikroanalyse hat ergeben, dass sich die Radverkehrsunfälle nach Unfallort in ihrer Charakteristik stark unterscheiden: Streckenunfälle weisen eine typische Charakteristik auf, die sich von Zwischenknotenpunktsunfällen stark unterscheidet. Die Unfälle an den Anfangs- und Endknotenpunkten waren aufgrund der unterschiedlichen örtlichen Gegebenheiten so heterogen,

16 Balken 2 und 1 (transparent) der Grafiken

17 Balken 3 der Grafiken

18 Balken 4 der Grafiken 
dass eine zusammenfassende Untersuchung hier nicht erfolgt ist, da dies einer Einzelfallbetrachtung entsprochen hätte.

Folglich wurden in einem weiteren Auswertungsschritt die mikroskopischen Ergebnisse aus der bundesweiten Untersuchung denen der Kölner Sonderuntersuchung in geöffneten Einbahnstraßen im Zwischenknoten und auf der Strecke gegenübergestellt (siehe Abbildung 13).

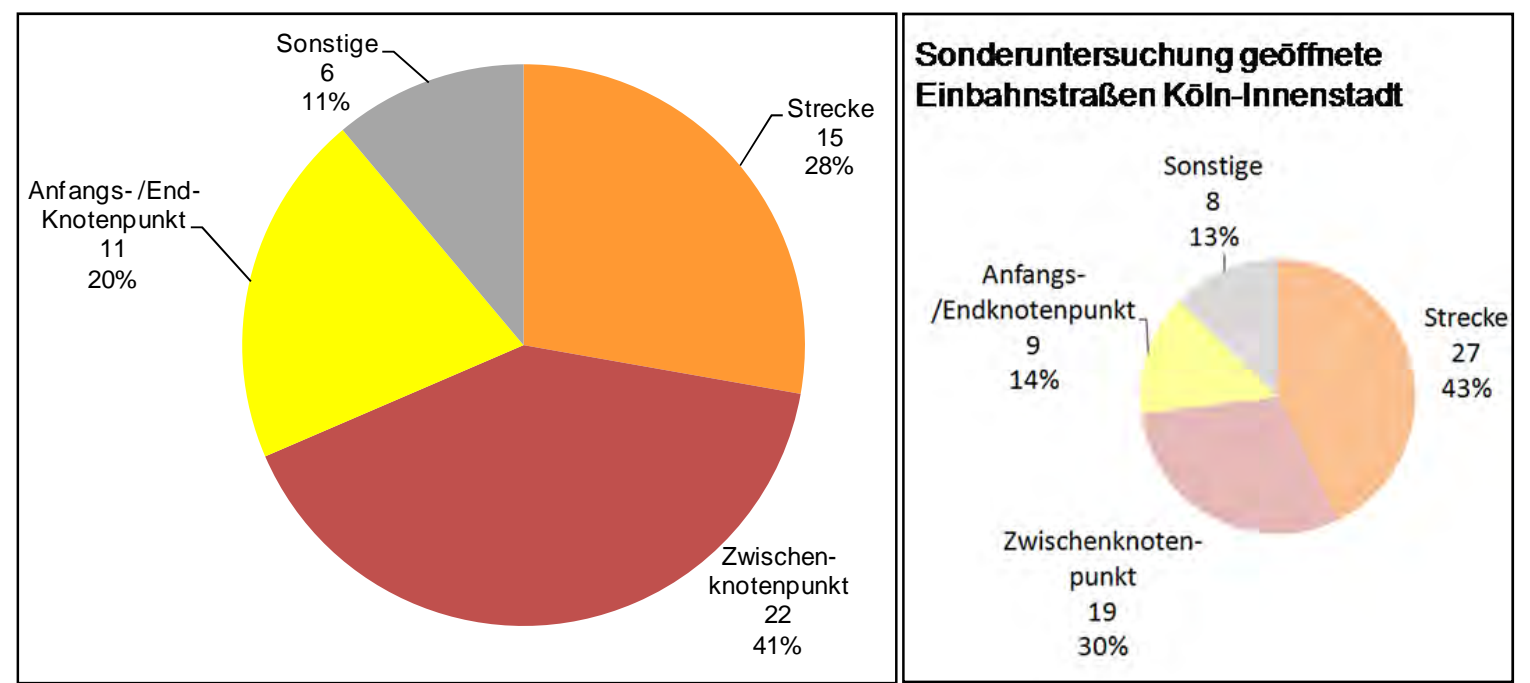

Abbildung 13: Unfallort der relevanten Radverkehrsunfälle in geöffneten Einbahnstraßen bundesweit und in der Sonderuntersuchung in der Kölner Innenstadt (Unfälle mit Personenschaden (U(P), 2008-2012) ( $n=54$ bzw. n=63)

In Kapitel 4.3 werden die Unfalldaten der untersuchten geöffneten Einbahnstraßen zu Unfallkategorie, Unfalltyp, Unfallart, Unfallort, Unfallbeteiligung und Unfallursachen ausgewertet und die Ergebnisse dargestellt.

Andere Parameter wie soziodemografische Daten wurden überprüft. Da die Ergebnisse unauffällig waren, wurde auf eine Darstellung verzichtet.

\subsection{Analyse und Beobachtung des Verkehrsverhaltens}

\section{Ziel der Videobeobachtung}

Ziel der Verhaltensbeobachtung war neben der Ermittlung der Verkehrsmengen und Geschwindigkeiten eine qualitative Verkehrssituationsanalyse zur Bewertung von Verkehrsabläufen, um die verkehrlichen Rahmendaten und die verhaltensspezifischen Merkmale der Verkehrsteilnehmer zu ermitteln. Die beiden Leitfragen im Zuge der Verhaltensbeobachtung waren:

- Wie verhalten sich die unterschiedlichen Verkehrsteilnehmer „Autofahrer“ und „Radfahrer" untereinander?

- Welche Konflikte sind vorhanden?

Die Auswertung der Erhebung sollte aufzeigen, ob es Gemeinsamkeiten im Verhalten der Verkehrsteilnehmer bei ähnlichen straßenräumlichen Situationen gab und welchen Einfluss die einzelnen Parameter auf das Verhalten der Verkehrsteilnehmer in der Fahrradstraße hatten. Für die geöffneten Einbahnstraßen wurde exemplarisch ausgewertet, ob sich an ausgewählten geöffneten Einbahnstraßen konflikthafte Situationen ergeben. 


\section{Aufbau der Videobeobachtung}

Grundsätzlich lag der Schwerpunkt der weiteren Untersuchung auf den Fahrradstraßen: 21 Untersuchungsorte in Fahrradstraßen sind in acht Städten festgelegt worden. Für die geöffneten Einbahnstraßen wurden fünf Untersuchungsorte in vier Städten ausgewählt.

Es sollten möglichst unfallbelastete Untersuchungsorte mit unfallunauffälligen verglichen werden. Aufgrund der insgesamt sehr geringen Anzahl von relevanten Unfällen an Knotenpunkten oder Streckenabschnitten wurden als unfallbelastet schon Knoten bzw. Strecken ab zwei Unfällen in fünf Jahren gewertet. Die absolut höchste Unfallbelastung an einem Knotenpunkt einer Fahrradstraße hatte ein Kreisverkehr mit acht relevanten Unfällen. Bei diesem Kreisverkehr handelte es sich allerdings auch um eine gestalterische Sonderform. Die zweithöchste Unfallbelastung wurde an einem weiteren Knotenpunkt derselben Fahrradstraße mit fünf relevanten Unfällen in fünf Jahren festgestellt. Insgesamt wies die betroffene Fahrradstraße die absolut meisten Unfälle auf, allerdings auch mit Abstand den höchsten Radverkehrsanteil von bis zu 12.000 Radfahrern am Tag.

Für die zu untersuchenden Knotenpunkte in Fahrradstraßen wurde neben dem Kriterium Unfallbelastung auch noch das Kriterium Vorfahrtregelung herangezogen, da untersucht werden sollte, warum Knotenpunkte mit gleicher Vorfahrtregelung und gleicher Charakteristik mit unterschiedlich vielen Unfällen belegt sind. Ähnlich wurde die Auswahl der Streckenabschnitte in Fahrradstraßen vorgenommen: Hier wurden unterschiedliche Breiten ausgewählt, um das Fahrverhalten und die Konflikte mit dem Parken untersuchen zu können.

Für die Auswahl der Knotenpunkte und Straßenabschnitte in Fahrradstraßen wurden folgende Kriterien berücksichtigt:

- Relevante Unfälle in vier Jahren (bis 1 Unfall: 7 Knotenpunkte / 2 und mehr Unfälle: 9 Knotenpunkte)

- Vorfahrtregelung (10 Knotenpunkte bevorrechtigt / 2 Knotenpunkte wartepflichtig / 4 Knotenpunkte Rechts-vor-links)

Für die geöffneten Einbahnstraßen wurden fünf Standorte ausgewählt, davon vier Knotenpunkte und ein Streckenabschnitt. Bei den geöffneten Einbahnstraßen wurden auch zwei Anfangs-I Endknotenpunkte untersucht, da bei den insgesamt sehr wenigen Unfällen an Knotenpunkten zwei Anfangs-/Endknotenpunkte als unfallbelastet bewertet wurden. Die Strecke wurde als zu untersuchende geöffnete Einbahnstraße ausgewählt, da nur hier relevante Unfälle auf einer Strecke festzustellen waren.

Die Videobeobachtung diente der Interaktionsanalyse, zur Ermittlung der Verkehrsstärken der Kfz und Radfahrer an allen Standorten, sowie zusätzlich auf den fünf Streckenabschnitten der Geschwindigkeitsmessung. Der Zeitraum der Beobachtung betrug an jedem Standort vier Stunden, die Videobeobachtung wurde von 7.00 Uhr bis $9.00 \mathrm{Uhr}$ sowie von $16.00 \mathrm{Uhr}$ bis 18.00 Uhr durchgeführt. Die Erhebung erfolgte im Juni 2014, als Erhebungstage wurden Normalwerktage (Dienstag bis Donnerstag) außerhalb der Ferienzeiten ausgewählt. Zudem wurde darauf geachtet, dass die Erhebungen an allen Standorten zu annähernd gleichen, günstigen Witterungsbedingungen durchgeführt wurden.

Aus den Daten der Videobeobachtung wurde für alle Standorte die Verkehrsstärke von Radfahrern und Kfz nach Art der Fahrzeuge (Krad, Pkw, Lkw,...) ermittelt und entsprechend den Fahrtrichtungen erfasst. Aus den Zählungen der beiden Zeitintervalle wurden anschließend die durchschnittlichen werktäglichen Verkehrsstärken (Fz./24h) für den Rad- und Kfz- Verkehr errechnet. Die Hochrechnung erfolgte für den Kfz-Verkehr nach den Regeln des Handbuchs für die 
Bemessung von Straßenverkehrsanlagen (HBS 2015). Für den Radverkehr kam das Verfahren der Technischen Universität Dresden (BMVBS 2011) zum Einsatz.

Die Geschwindigkeiten von Kfz wurden aus den Videos ermittelt und mit Hilfe von Stoppuhren gemessen. Aus den ermittelten Daten wurde die $V_{85}$-Geschwindigkeit ermittelt.

\section{Interaktionsanalyse}

Für die Analyse des Verhaltens im Begegnungsfall von Radfahrern und Autofahrern wurden vorab die Begriffe Interaktion und Konflikt definiert. Ein Begegnungsfall ist dann gegeben, wenn ein Radfahrer und ein Kfz zeitgleich eine Kreuzung oder einen Streckenabschnitt befahren. Dabei ist es unerheblich, ob die beiden Fahrzeuge auf Streckenabschnitte in gleicher Richtung oder entgegen gesetzt fahren bzw. an Kreuzungen unabhängige oder feindliche Fahrlinien haben. In Abbildung 14 ist die Systematik aufbereitet.

\begin{tabular}{|c|c|c|c|c|}
\hline \multicolumn{5}{|c|}{ Begegnungsfälle } \\
\hline \multicolumn{4}{|c|}{ Mit Interaktion } & Ohne Interaktion \\
\hline \multicolumn{3}{|c|}{ Mit Konflikt } & Ohne Konflikt & \\
\hline \multicolumn{2}{|c|}{$\begin{array}{l}\text { Schwerer } \\
\text { Konflikt }\end{array}$} & $\begin{array}{l}\text { Leichter } \\
\text { Konflikt }\end{array}$ & & \\
\hline Undall & $\begin{array}{l}\text { Ohrie } \\
\text { Unfall }\end{array}$ & & & \\
\hline
\end{tabular}

Abbildung 14: Systematik der Begegnungsfälle

Interaktion: Ein Begegnungsfall wurde als Interaktion gewertet, sobald das Aufeinandertreffen von Radfahrer und Autofahrer eine Abstimmung ihres Verhaltens erfordert, damit der weitere Verlauf der Begegnung konfliktfrei verläuft. Für die fünf beobachteten Streckenabschnitte wurde aufgrund der geringen Straßenbreite auch eine Begegnung von Rad- und Kfz-Fahrern, die in entgegengesetzter Richtung fahren, als Interaktion gewertet.

Konflikt: Eine Interaktion wird zum Konflikt, wenn zumindest ein Verkehrsteilnehmer eine auffällige Verhaltensanpassung vornimmt, um eine Kollision zu vermeiden. In der Analyse wurde zwischen konfliktfreien Interaktionen sowie zwischen leichten und schweren Konflikten unterschieden.

Leichte Konflikte bedingen eine kontrollierte Verhaltungsanpassung zumindest eines Verkehrsteilnehmers wie bspw. leichtes Abbremsen oder seitliches Ausweichen.

Schwere Konflikte erfordern in der Regel ein deutliches und abruptes Vermeidungsmanöver (starkes Bremsen/deutliches Ausweichen) seitens des Rad- und/oder des Kfz-Fahrers, um eine Kollision zu vermeiden.

Konfliktfreie Interaktion beinhalten auch Momente der erhöhten Achtsamkeit, bspw. während eines Überholvorganges. Auch das Zusammentreffen an Kreuzungspunkten gilt als konfliktfreie Interaktion, sofern der wartepflichtige Verkehrsteilnehmer durch frühzeitiges Reagieren (Anhalten 
bzw. langsamer fahren) dem Vorfahrtberechtigten seine Absicht signalisiert und dieser seine Fahrt ungehindert fortsetzten kann.

\subsection{Befragung von Verkehrsteilnehmern}

Neben der Verhaltensbeobachtung wurde zusätzlich an ausgewählten Fahrradstraßen eine Verkehrsteilnehmerbefragung durchgeführt. Ziel der Befragung war es, das Wissen über das regelkonforme Verhalten in Fahrradstraßen sowie das subjektive Sicherheitsgefühl der Verkehrsteilnehmer in Fahrradstraßen zu ermitteln. Die Antworten wurden zu den straßenräumlichen Gegebenheiten, sowie zu den Ergebnissen der Interaktionsanalyse und der Unfallanalyse in Beziehung gesetzt.

Die Auswahl der Standorte zur Befragung erfolgte ebenso wie bei der Videobeobachtung auf der Basis der in der Kommunalbefragung genannten Fahrradstraßen sowie der Unfallanalyse. Die Interviews wurden an zehn Standorten in sechs Städten mit insgesamt 452 Verkehrsteilnehmern durchgeführt.

Befragt wurden sowohl Radfahrer als auch Fußgänger und Autofahrer, welche gerade von ihrem Fahrzeug kamen oder auf dem Weg zu ihrem Fahrzeug waren, wobei sich der Fragebogen inhaltlich nicht zwischen den Teilnehmergruppen unterschied.

Der Fragebogen ist als Anhang 4 beigefügt.

\subsection{Ableitung von Empfehlungen}

Abschließend werden für beide Infrastrukturelemente Empfehlungen für deren Einsatz und Gestaltung abgeleitet. Bei den Fahrradstraßen liegt der Fokus dabei auf Aussagen mit Bezug zu Dimensionierung und Gestaltung. Bei den Einbahnstraßen steht die Frage im Vordergrund, ob sich über das bestehende Repertoire für die Öffnung von Einbahnstraßen hinaus noch Handlungsbedarf ableiten lässt, um geöffnete Einbahnstraßen noch sicherer zu gestalten. 


\section{Fahrradstraßen}

\subsection{Literaturauswertung}

\subsubsection{Definitionen, rechtliche Grundlagen und Planungsrichtlinien}

Die rechtlichen Grundlagen für die Einrichtung von Fahrradstraßen sind in der StraßenverkehrsOrdnung (StVO) und der Verwaltungsvorschrift zur StVO (VwV-StVO) festgelegt. Hinweise zur Einrichtung von Fahrradstraßen finden sich außerdem in den Richtlinien zur Anlage von Stadtstraßen (RASt 06) und den Empfehlungen für Radverkehrsanlagen (ERA 2010).

Die VwV-StVO (BMVI 2015) führt aus, wann eine Fahrradstraße zulässig ist:

„Fahrradstraßen können eingerichtet werden, wenn der Radverkehr die vorherrschende Verkehrsart ist oder dies alsbald zu erwarten ist. Sie sind mit dem Zeichen 244.1 StVO („Beginn einer Fahrradstraße“) bzw. Zeichen 244.2 StVO („Ende einer Fahrradstraße“) auszuweisen.“

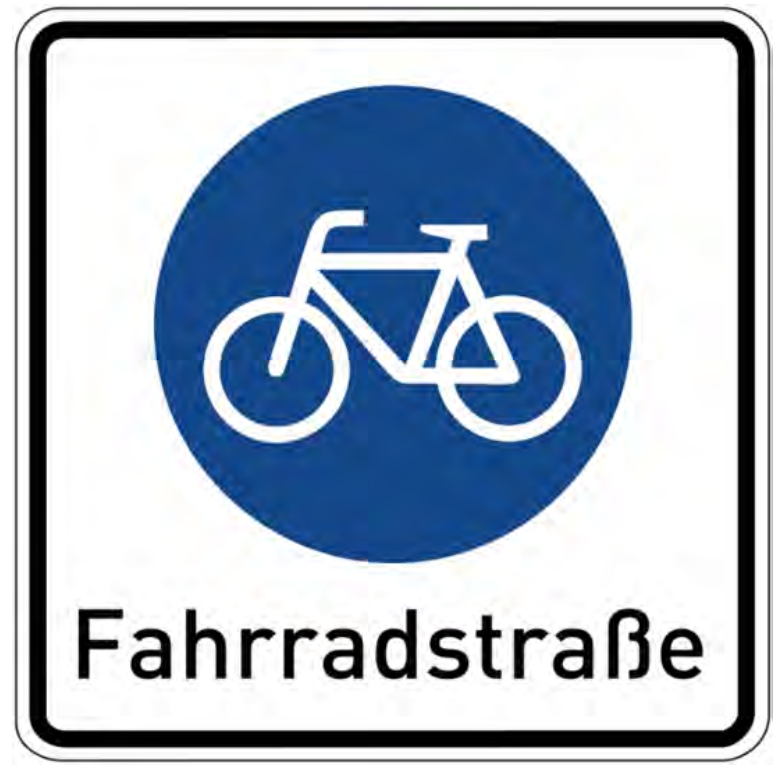

Abbildung 15: Beginn einer Fahrradstraße, Zeichen 244.1 StVO

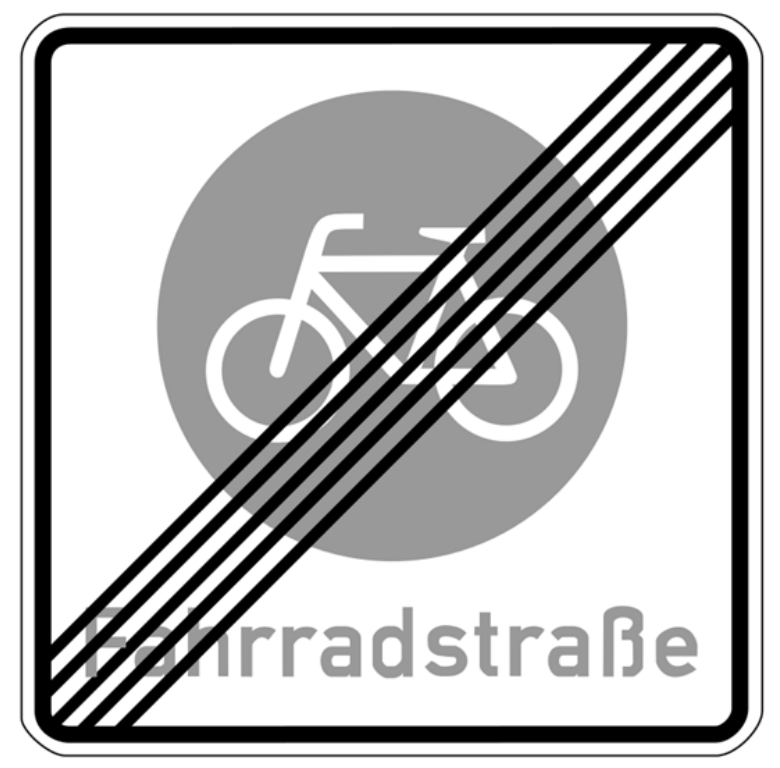

Abbildung 16: Ende einer Fahrradstraße, Zeichen 244.2 StVO

Grundsätzlich sind Fahrradstraßen dem Radverkehr vorbehalten, solange nicht weitere Verkehre durch Zusatzschilder, wie z.B. 1020-30 „Anlieger frei“, 1022-12 „Krafträder frei“ oder 1024-10 „Personenkraftwagen frei“, zugelassen werden (siehe Abbildung 17). Es gilt eine Höchstgeschwindigkeit von 30 km/h für den Fahrverkehr. Eine Fahrradstraße kann je nach örtlicher Situation in beide oder nur eine Richtung für $\mathrm{Kfz}$ freigegeben werden. In jedem Fall darf der Radverkehr vom Kraftfahrzeugverkehr nicht gefährdet oder behindert werden. Im Zweifelsfall muss ein Kfz-Fahrer die Geschwindigkeit weiter verringern (VwV-StVO). Grundsätzlich gelten die gleichen Vorschriften zur Fahrbahnnutzung und Vorfahrtsregelung wie in anderen Straßen. Entgegen § 2 Absatz 4 StVO ist in Fahrradstraßen jedoch das Nebeneinanderfahren von Radfahrern erlaubt. Trotzdem ist das Rechtsfahrgebot einzuhalten ( 2 Absatz 2 StVO). 


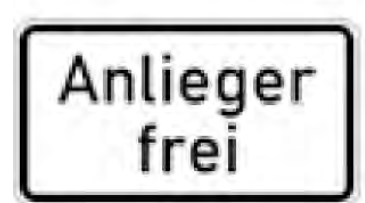

Zeichen 1020-30

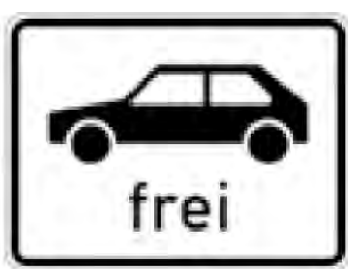

Zeichen 1024-10

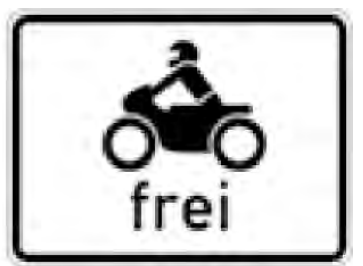

Zeichen 1022-12

Abbildung 17: Beispiele für die Zulassung anderer Verkehrsarten in Fahrradstraßen

Fahrradstraßen sollten vor allem für Hauptverbindungen des Radverkehrs und bei hohem Radverkehrsaufkommen eingesetzt werden.

Gemäß den RASt sollen Fahrradstraßen an Knotenpunkten Vorfahrt gegenüber anderen Erschließungsstraßen erhalten. Die ERA führen dazu weiter aus, dass die Entscheidung über die Vorfahrt auch von örtlichen Gegebenheiten abhängig gemacht werden sollte (z.B. an Knotenpunkten mit Hauptverkehrsstraßen). In den ERA 2010 wird weiterhin ausgeführt, dass Fahrradstraßen gegenüber anderen Erschließungsstraßen bevorrechtigt werden können, um eine kontinuierliche Geschwindigkeit des Radverkehrs zu ermöglichen. In diesem Fall sind in Abhängigkeit der örtlichen Gegebenheiten unter Umständen Maßnahmen zu treffen, welche die Geschwindigkeiten des Kraftfahrzeugverkehrs wirksam reduzieren, damit diese nicht auch für den $\mathrm{Kfz}$-Durchgangsverkehr attraktiv wird. Weiterhin sollte die Bevorrechtigung durch eine entsprechende bauliche Gestaltung der Kreuzung verdeutlicht werden, z.B. durch die Anhebung der Fahrradstraße im Vergleich zur kreuzenden Erschließungsstraße. Zudem empfiehlt es sich, die Fahrradstraße an Einmündungen durch Fahrradpiktogramme zu kennzeichnen sowie optional auch eine bauliche Einengung der einmündenden Straße vorzunehmen.

\subsubsection{Ergebnisse der Literaturrecherche}

Die Literaturrecherche ergab insgesamt eine Vielzahl an Quellen zu Fahrradstraßen. Das Hauptaugenmerk lag dabei auf Veröffentlichungen zur Verkehrssicherheit sowie zur Wirksamkeit und Wahrnehmung von Fahrradstraßen.

Bei den wenigsten Quellen handelt es sich jedoch um wissenschaftlich fundierte Literatur. Die konkrete Verbreitung von Fahrradstraßen in Deutschland konnte aus den veröffentlichten Ergebnissen nicht abgeleitet werden und es zeigte sich, dass aktuelle Untersuchungen und fundierte Erkenntnisse zur Verkehrssicherheit in Fahrradstraßen nicht vorhanden sind.

Im Folgenden werden wesentliche Erkenntnisse aus der Literaturrecherche zu folgenden Aspekten dargestellt:

- Einsatz von Fahrradstraßen

- Verhalten und Konflikte in Fahrradstraßen

- Öffentlichkeitsarbeit für Fahrradstraßen

\section{Einsatz von Fahrradstraßen}

Ein gesamtstädtisches Fahrradstraßenkonzept im Rahmen des Verkehrsentwicklungsplans 2020 hat die Stadt Bonn im Jahr 2012 vorgestellt (STADT BONN 2012). Das Konzept gibt eine Netzübersicht möglicher Fahrradstraßen und legt Gestaltungsstandards für Fahrradstraßen fest. Bei den bisherigen sechs vorhandenen Fahrradstraßen wird bemängelt, dass diese aufgrund ihrer Gestaltung teilweise von den Kfz-Fahrern nicht in ihrer Bedeutung erkannt werden. Zum anderen werden die oft hohen Geschwindigkeiten sowie das regelwidrige Halten von Fahrzeugen auf den 
Fahrradstraßen kritisiert. Die neuen Fahrradstraßen sollen neben der Verbindungs- und Sammelfunktion auch eine Erschließungs- und Anbindungsfunktion zu Schulen und Stadtteilzentren einnehmen. Insgesamt sollen rund 52 Kilometer neue Fahrradstraßen entstehen. Die wichtigsten Gestaltungspunkte des Konzeptes sind:

- Neben der obligatorischen Beschilderung soll aufgrund der geringen Akzeptanz von Fahrradstraßen durch Kraftfahrer zumindest bei Einrichtungsverkehr des Kfz-Verkehrs das Verbot der Einfahrt zusätzlich gekennzeichnet werden.

- Fahrradstraßen sollen durch deutliche Fahrbahnmarkierungen hervorgehoben werden. Hierfür werden zwei nebeneinanderliegende Fahrradpiktogramme mit entgegengesetzten Fahrtrichtungspfeilen mittig auf der Fahrbahn empfohlen, die über Beginn und Ende der Strecke hinaus alle $50 \mathrm{~m}$ markiert werden sollen.

- Im Verlauf der Strecke soll die Straße eine unterbrochene Breitstrichmarkierung erhalten, welche eine Fahrgasse von 3,00 bis 4,50 m für die Radfahrer markiert.

- Zur Hervorhebung des Beginns der Fahrradstraße sollen bauliche Maßnahmen im Einmündungsbereich erfolgen, wie bspw. Pflasterbänder oder Teilaufpflasterungen.

- Vorgesehen sind werden auch ein- oder beidseitig vorgezogene Seitenräume am Beginn der Fahrradstraße, da diese aufgrund ihrer Kanalisierung einen verkehrsberuhigenden Effekt haben.

- Besonders wichtige Hauptverbindungen sollen in Knotenpunkten Vorfahrt erhalten, der Standardfall soll allerdings die Rechts-vor-links-Regelung sein. Zur Verdeutlichung der Vorfahrtregelungen sollen Wartelinien markiert werden. Bei den vorfahrtberechtigten Fahrradstraßen wird als Maßnahme zur Geschwindigkeitsreduzierung ggf. die Fahrbahnanhebung im Kreuzungsbereich empfohlen.

- Da angenommen wird, dass der Großteil der Verkehrsteilnehmer die Regeln in einer Fahrradstraße nicht kennen, ist eine umfassende Öffentlichkeitsarbeit vorgesehen, bestehend aus Pressemitteilungen, feierlichen Eröffnungen, Informationsflyern und Plakataktionen.

Die Stadt Bremen hat 2015 ebenfalls eigene Leitlinien für die Gestaltung von Fahrradstraßen im Rahmen des Verkehrsentwicklungsplans Bremen 2025 (STADT BREMEN 2015) erarbeitet. Der Plan sieht acht weitere Fahrradstraßen vor, die allesamt Teil des Hauptradroutennetzes sein sollen.

Für die Gestaltung der Fahrradstraßen gelten folgende Grundsätze:

- Oberhalb einer Verkehrsbelastung von $5.000 \mathrm{Kfz} / \mathrm{Tag}$ sollen keine Fahrradstraßen eingerichtet werden.

- Eine Mindestbreite von 4,50 Metern soll nicht unterschritten werden.

- Senkrecht- und Schrägparkstände sollen generell vermieden werden.

- Fahrradstraßen sollen grundsätzlich vorfahrtberechtigt sein.

- Einmündungen sollen möglichst nur für abbiegende, d. h. aus der Fahrradstraße(ausfahrende) Kfz ausgelegt werden.

- Gehwegüberfahrten über die einmündenden Straßen hinweg sollen den Vorrang der Fahrradstraße zusätzlich betonen. 
- Gestaltung durch Beschilderung in Kombination mit extra großen Fahrradpiktogrammen $(1,50 \mathrm{~m} \times 1,95 \mathrm{~m})$ zu Beginn der Fahrradstraße und im Bereich von einmündenden Straßen, im Streckenverlauf kleinere Piktogramme von 1,00 m x 1,30 m.

- Entflechtung des Radverkehrs vom ein- bzw. ausfahrenden Kfz-Verkehr im Bereich der Zufahrten des Radverkehrs durch Ausbildung von kurzen Schutzstreifen.

- Um hohe Radfahr-Geschwindigkeiten erreichen zu können, soll die Fahrradstraße i.d.R. asphaltiert sein.

- Auf Aufpflasterungen soll im Fahrbahnquerschnitt verzichtet werden, nach Möglichkeit auch auf Ampelanlagen und Fußgängerüberwege („Zebrastreifen“).

- Im Bereich der Ausfahrten ggf. Errichtung von Fahrradschutzstreifen und vorgezogene Aufstellflächen vor Ampeln.

- Öffentlichkeitsarbeit durch Pressemitteilungen und Faltblätter soll die Einrichtung einer Fahrradstraße ankündigen und begleiten.

Dies sind zwei Beispiele für die Anwendung der Fahrradstraßen vor Ort, wobei die sehr allgemeinen Vorgaben aus den Regelwerken durch die kommunalen Akteure ergänzt und entsprechend den örtlichen Verhältnissen konkretisiert wurden.

\section{Verhalten und Konflikte in Fahrradstraßen}

Im Rahmen des Modellvorhabens „Fahrradfreundliche Städte und Gemeinden in NRW“ hat die Stadt Münster 1990 die Schillerstraße als Fahrradstraße ausgewiesen. 1993 wurden die Ergebnisse der Begleituntersuchung veröffentlicht (STADT MÜNSTER 1993). Entscheidend für die Beurteilung war die erreichte Verkehrssicherheit. Neben einer Verkehrsmengenzählung fanden auch eine Videobeobachtung des Verkehrsgeschehens sowie eine Befragung der Radfahrer statt. Zusätzlich wurden die Unfallzahlen vor und nach der Einführung der Fahrradstraße mit positivem Ergebnis verglichen. Bei der Auswertung des Verkehrsgeschehens wurden folgende Beobachtungen gemacht:

- Die größte Anzahl an Konflikten wurde im Rahmen von Überholvorgängen beobachtet (nutzbare Fahrbahnbreite von 3,85 m, mit beidseitig markierten Parkstreifen). Teilweise wurden die Radfahrer so dicht und schnell überholt, dass sie zum Ausweichen gezwungen wurden.

- Ebenfalls ergaben sich Konflikte im Begegnungsverkehr Rad/ Kfz. Radfahrer wurden durch das entgegenkommende $\mathrm{Kfz}$ veranlasst abzubremsen oder auszuweichen.

- Ein Konflikt wurde im Zuge eines Einparkmanövers beobachtet, bei dem der Radfahrer gezwungen war auszuweichen.

- In der Auswertung zeigte sich, dass $42 \%$ der $\mathrm{Kfz}$ zu schnell fuhren $\left(\mathrm{V}_{85}=38,1 \mathrm{~km} / \mathrm{h}\right)$.

- In den Zeiten mit Schülerverkehr, bei denen die Radfahrer im Pulk fuhren, wurden keine Konflikte beobachtet.

Im Ergebnis wurde die Verkehrssicherheit der Fahrradstraße in der Untersuchung als hoch bewertet, für Radfahrer zeigte sich ein nur geringes Konfliktrisiko. Demgegenüber zeigte die Verkehrsteilnehmerbefragung, dass sich rund die Hälfte der Radfahrer durch zu schnell fahrende und zu dicht überholende $\mathrm{Kfz}$ verunsichert fühlte. Als Handlungsempfehlungen wurden eine bessere Beschilderung sowie Maßnahmen zur Geschwindigkeitsreduzierung abgeleitet.

Eine Untersuchung der BASt aus dem Jahr 1997 zum Thema „Sicherheit des Radverkehrs auf Erschließungsstraßen“ (ALRUTZ \& STELLMACHER-HEIN: 1997) bewertet die Verkehrssicherheit 
von Fahrradstraßen ebenfalls positiv. In der Unfallanalyse zeigte sich, dass die Anzahl der Konflikte, vor allem zwischen Kfz und Rad, nach der Einrichtung der Fahrradstraßen rückläufig war. Jedoch wird in der Untersuchung einschränkend daraufhin hingewiesen, dass die geringe Anzahl von drei untersuchten Fahrradstraßen für aussagekräftige Ergebnisse zum Unfallgeschehen nicht ausreichend ist. In der Untersuchung wurden folgende Beobachtungen gemacht:

- Konfliktsituationen entstanden oftmals in Verbindung mit Überholvorgängen an punktuellen Einengungen durch den ruhenden Verkehr, wodurch der Seitenabstand beim Überholen einen kritischen Wert von unter einem Meter erreichte.

- Die Geschwindigkeitsmessungen zeigten eine deutliche Überschreitung der zulässigen Geschwindigkeiten $\left(V_{85}=40 \mathrm{~km} / \mathrm{h}\right)$.

- Die Vorrangregelung der Fahrradstraße wurde bei klarer Kennzeichnung von den KfzFahrern gut beachtet, lediglich bei $6 \%$ der Interaktionen wurde sie missachtet.

- Es zeigten sich deutliche Unterschiede zwischen baulich (Aufpflasterung) und lediglich durch Markierung gekennzeichneten Querungsstellen.

- Bei Radfahrern war generell ein defensives Fahrverhalten zu beobachten, mit der Bereitschaft zum freiwilligen Verzicht auf den eigenen Vorrang.

- Die Befragung der Verkehrsteilnehmer machte deutlich, dass die Kenntnis von der rechtlichen Bedeutung der in Fahrradstraßen geltenden Regelungen sehr unpräzise war, bei Autofahrern jedoch etwas besser als bei Radfahrern. Gleichzeitig gab es überwiegend Zustimmung zu Fahrradstraßen seitens der Verkehrsteilnehmer.

Aus ihren Beobachtungen leiten ALRUTZ \& STELLMACHER-HEIN (1997) folgende Gestaltungsempfehlungen ab:

- Eindeutige Kennzeichnung der Fahrradstraßen durch Beschilderung und Markierung von Fahrradpiktogrammen an allen Knotenpunkten.

- Punktuelle bauliche Maßnahmen zur Geschwindigkeitsreduzierung im Bereich der Knotenpunkte, bspw. durch Aufpflasterungen.

- Bauliche Umgestaltung von Knotenpunkten, in denen die Fahrradstraße bevorrechtigt geführt werden soll (Aufpflasterungen sowie Materialwechsel zur Kennzeichnung der möglichen Konfliktstelle für die Radfahrer).

In der Begleitforschung der Maßnahmen- und Wirksamkeitsuntersuchung der Fahrradfreundlichen Städte und Gemeinden in NRW (PS \& PGV: 2000) wurden Fahrradstraßen als schnell realisierbare und kostengünstige Maßnahme beschrieben, welche eine erhebliche Verbesserung der Fahrbeziehungen bewirken und zu einer generellen Erhöhung des Radverkehrsaufkommens führen. Die Erfahrungen der Städte in NRW mit den Fahrradstraßen wurden hier durchweg als positiv in Bezug auf die Verkehrssicherheit beschrieben. In der Untersuchung wurden den Fahrradstraßen zudem eine sehr positive Bewertung seitens der Bevölkerung sowie eine hohe Öffentlichkeitswirksamkeit attestiert.

In einer aktuelleren Untersuchung von DELBRESSINE (2013) wurden acht Fahrradstraßen in den Niederlanden im Hinblick auf ihre Verkehrssicherheit untersucht. Zu diesem Zweck fand neben einer Verkehrszählung auch eine Verhaltensbeobachtung statt. Die wichtigsten Erkenntnisse sind:

- Kfz-Fahrer halten sich mehrheitlich nicht an die Geschwindigkeitsbegrenzung von $30 \mathrm{~km} / \mathrm{h}$. 
- Das Mengenverhältnis von Radfahrenden zu Kfz-Fahrern hat keinen Einfluss auf das Geschwindigkeitsniveau. Vielmehr ist die zur Verfügung stehende Fahrbahnbreite ausschlaggebend.

- Auf der Fahrbahn parkende oder anhaltende Kfz sind grundsätzlich ein Hindernis und damit eine mögliche Konfliktquelle für den Radverkehr.

Auch DELBRESSINE gibt Empfehlungen, die bei der Einrichtung von Fahrradstraßen berücksichtigt werden sollten. Dazu zählt:

- Ein einheitliches Design der Fahrradstraßen sollte den Wiedererkennungswert erhöhen.

- Fahrradstraßen sollten eine Mindestbreite von 4,00 m in Einbahnstraßen, bzw. 4,50 m bei Gegenverkehr aufweisen. Eine maximale Breite von 5,00 m sollte jedoch nicht überschritten werden, da die Kfz-Geschwindigkeiten sonst deutlich zunehmen.

- Die Fahrgasse für Radfahrer sollte durchgängig mit einem roten Asphaltband in der Mitte der Fahrbahn markiert werden.

- Um Konflikten mit dem ruhenden Verkehr vorzubeugen, sollte eine zusätzliche „Sicherheitszone“ zu den am Rand parkenden Kfz hin eingerichtet werden, die ebenfalls farblich markiert ist.

- An Knotenpunkten sollten bauliche Maßnahmen getroffen werden, um die Geschwindigkeit der Kfz deutlich zu reduzieren. Dies kann z. B. durch eine Aufpflasterung des Knotenpunktes erfolgen.

- An Knotenpunkten sollte der Verlauf der Fahrradstraße zudem farblich durch das o. g. durchgängige rote Asphaltband markiert sein.

- Wichtig ist zudem eine gut sichtbare und eindeutige Beschilderung.

Auf dem Nationalen Radverkehrskongress in Münster 2013 wurde als Diskussionsergebnis u.a. festgehalten, dass keine generellen Aussagen zur Verkehrssicherheit in Fahrradstraßen gemacht werden können, sondern dass Städte vielmehr ein flächenhaftes Unfallgeschehen mit einigen Risikobereichen aufweisen. So konnten bspw. in Münster keine Auffälligkeiten durch die Einrichtung von Fahrradstraßen festgestellt werden (BMVBS: 2013).

Aufgrund der sehr kleinen Stichproben der vorgestellten Untersuchungen lassen sich keine allgemeingültigen Erkenntnisse bezüglich der Verkehrssicherheit ableiten, jedoch liegen in den untersuchten Fällen auch keine Unfallauffälligkeiten vor. Die Ergebnisse der zitierten Studien fließen insbesondere in die Arbeitspakete 3 und 4 dieses Forschungsvorhabens mit ein.

\section{Öffentlichkeitsarbeit für Fahrradstraßen}

Weit verbreitet sind Informationen über neu eingerichtete Fahrradstraßen und Ratgeber zum Verhalten in Fahrradstraßen wie in Abbildung 18 dargestellt. Herausgeber sind neben öffentlichen Verwaltungen auch Interessenverbände wie ADFC oder VCD. Im Zuge von neu eingerichteten Fahrradstraßen verteilen viele Kommunen im Rahmen der Öffentlichkeitsarbeit Flyer, welche auf die neue Fahrradstraße hinweisen und die Vorteile für den Radverkehr sowie die spezifisch geltenden Vorschriften erläutern. 

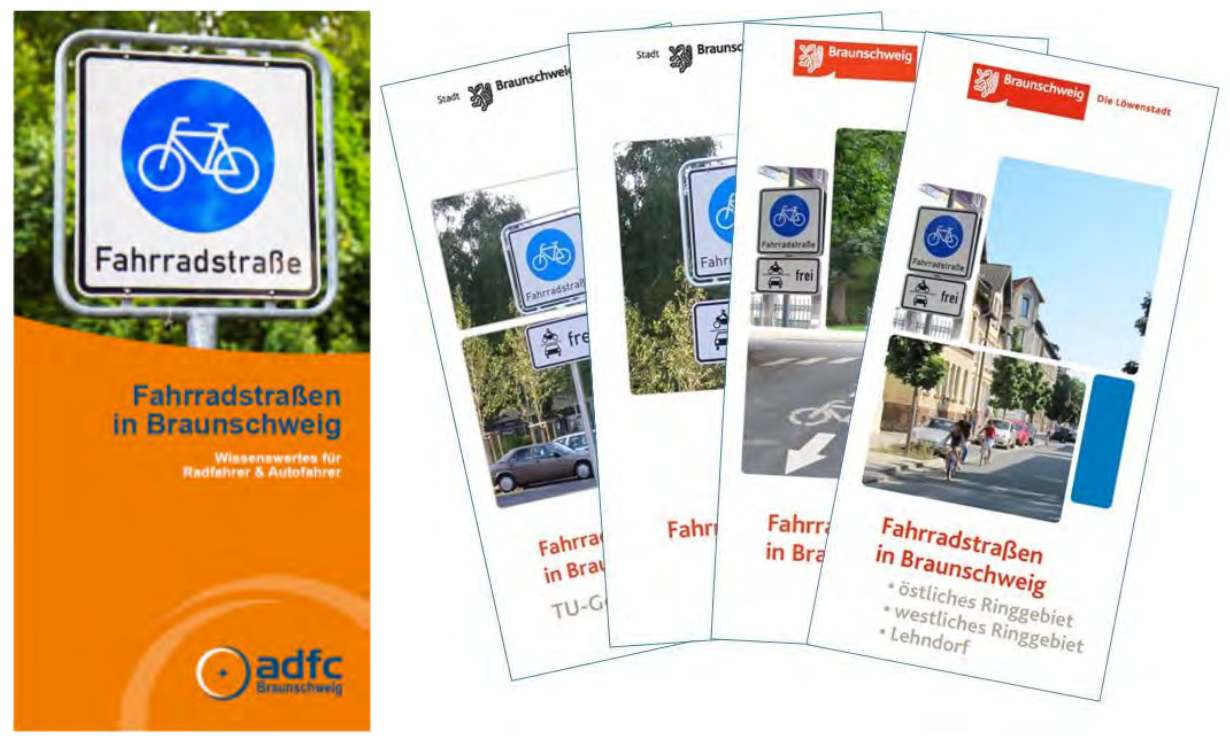

Abbildung 18: Beispiele für Flyer zum Thema „Fahrradstraßen“ (ADFC BRAUNSCHWEIG/STADT BRAUNSCHWEIG)

Weitgehend unbekannt ist, ob und wie weitreichend die Regelkenntnis der Verkehrsteilnehmer zur Nutzung von Fahrradstraßen ist. Dazu liegen keine spezifischen deutsch- oder englischsprachigen Untersuchungen veröffentlicht vor. Hier besteht demnach Untersuchungsbedarf.

\subsection{Ergebnisse der kommunalen Befragung}

\subsubsection{Auswertung der Befragung}

\section{Verbreitung von Fahrradstraßen}

Da es bislang keine Erkenntnisse über die bundesweite Verbreitung von Fahrradstraßen gibt, wurde in Phase I der Befragung ermittelt, welche Kommunen Fahrradstraßen eingerichtet haben. In 110 der 359 an der Befragung teilnehmenden Kommunen (31\%) waren insgesamt 426 Fahrradstraßen innerorts ausgewiesen. Dabei reicht die Spannweite von einer bis 47 Fahrradstraßen pro Kommune.

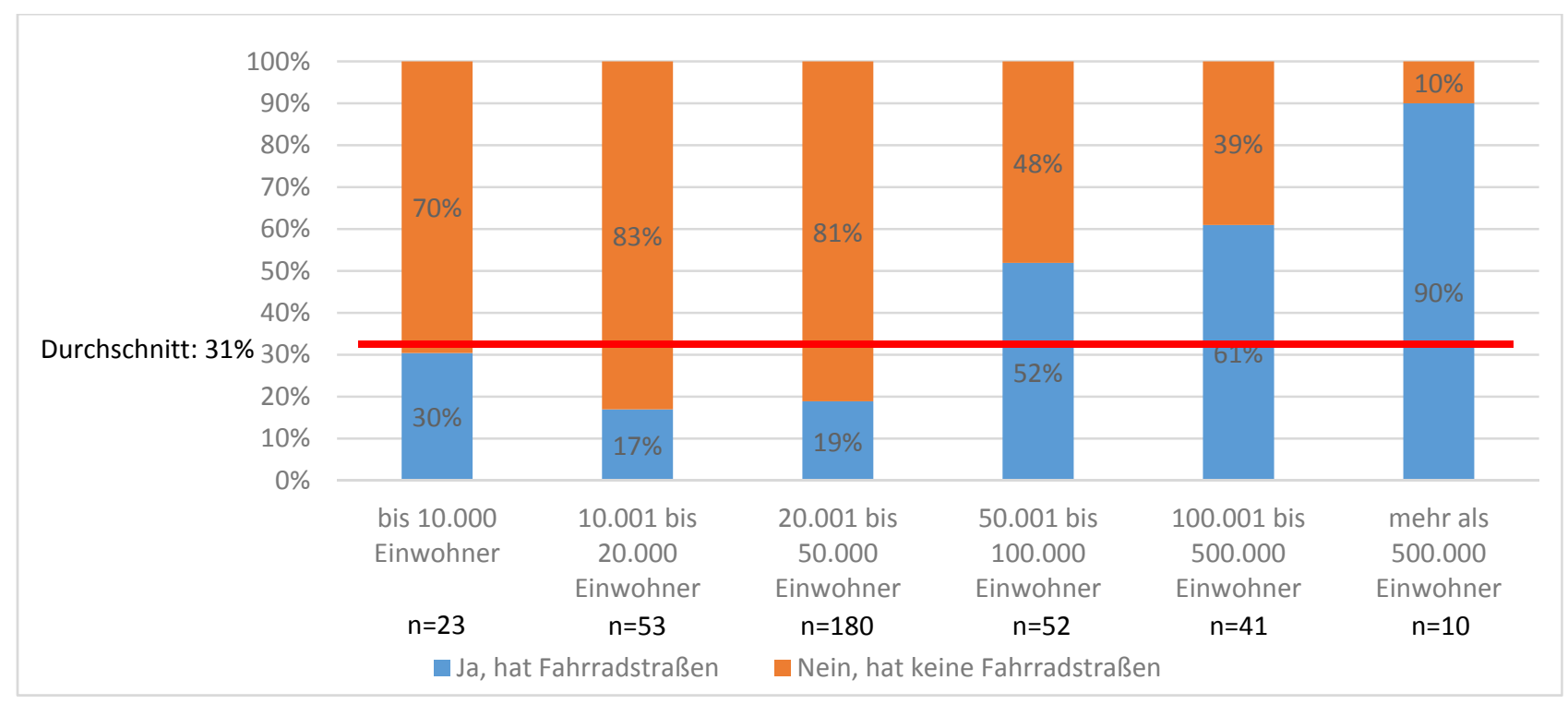

Abbildung 19: Einsatz von Fahrradstraßen nach Kommunengröße (n=359 Kommunen) 
Betrachtet man die Angaben in den einzelnen Kommunenklassen, zeigt sich ein heterogenes Bild (Abbildung 19):

- Kleinstädte und Landgemeinden mit bis zu 10.000 Einwohnern wiesen gegenüber den beiden nächstgrößeren Kommunenklassen (10.000 bis 20.000. und 20.000 bis 50.000 Einwohner) auffällig mehr Fahrradstraßen aus.

- In Kommunen, die zwischen 10.000 und 50.000 Einwohner haben, waren Fahrradstraßen eher gering verbreitet, jeweils weniger als $20 \%$ der Kommunen haben Fahrradstraßen ausgewiesen.

- In Städten mit einer Einwohnerzahl von mehr als 50.000 fanden sich deutlich häufiger Fahrradstraßen als in kleinen Mittelstädten zwischen 10.000 und 50.000 Einwohnern (Faktor 2,5).

- Mit Ausnahme einer Stadt wurden in 9 der 10 Großstädte ab 500.000 Einwohnern Fahrradstraßen eingesetzt.

Bei der Frage nach der zukünftigen Einrichtung von Fahrradstraßen zeigte sich ein deutlicher Unterschied zwischen den Kommunen, die bereits Fahrradstraßen ausgewiesen haben $(n=110)$ und jenen, die noch keine ausgewiesen haben $(n=243)$. So konnten sich $84 \%(n=92)$ der Kommunen mit Fahrradstraßen vorstellen, weitere Fahrradstraßen auszuweisen, während es bei den Kommunen ohne Fahrradstraßen lediglich $37 \%$ ( $n=89)$ waren (Abbildung 20).

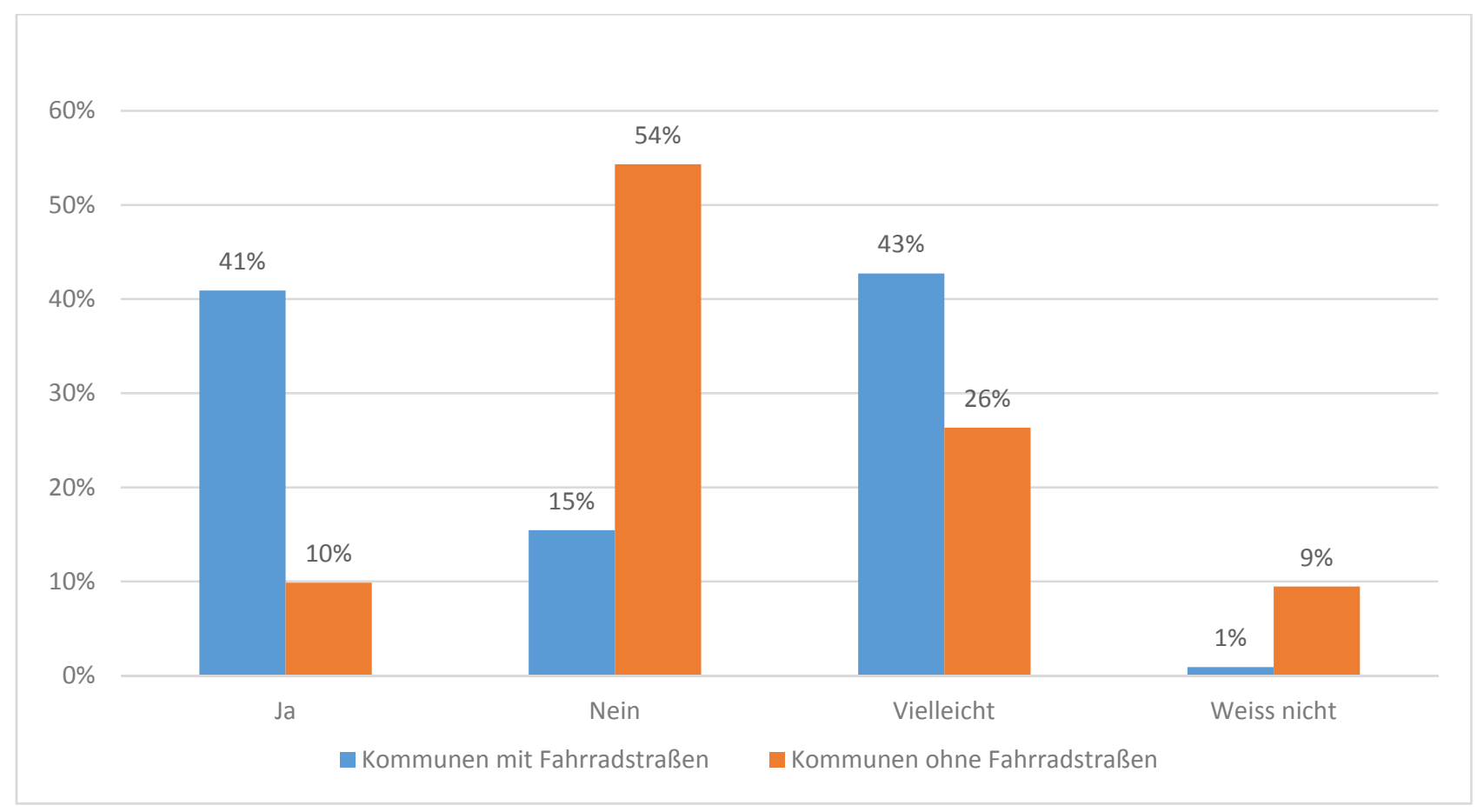

Abbildung 20: $\quad$ Geplante Einrichtung von Fahrradstraßen in den befragten Kommunen in \% ( $n=353)$

Bei den 130 Kommunen, die keine Fahrradstraßen einsetzen und dies auch in Zukunft nicht planen, wurde nach den Gründen für diese Entscheidung gefragt (Abbildung 21). Vorrangig handelte es sich um Kommunen bis 50.000 Einwohner, die Gründe gegen die Ausweisung von Fahrradstraßen vorbringen, 121 Kommunen stammen aus dieser Gruppe (93 \%). 


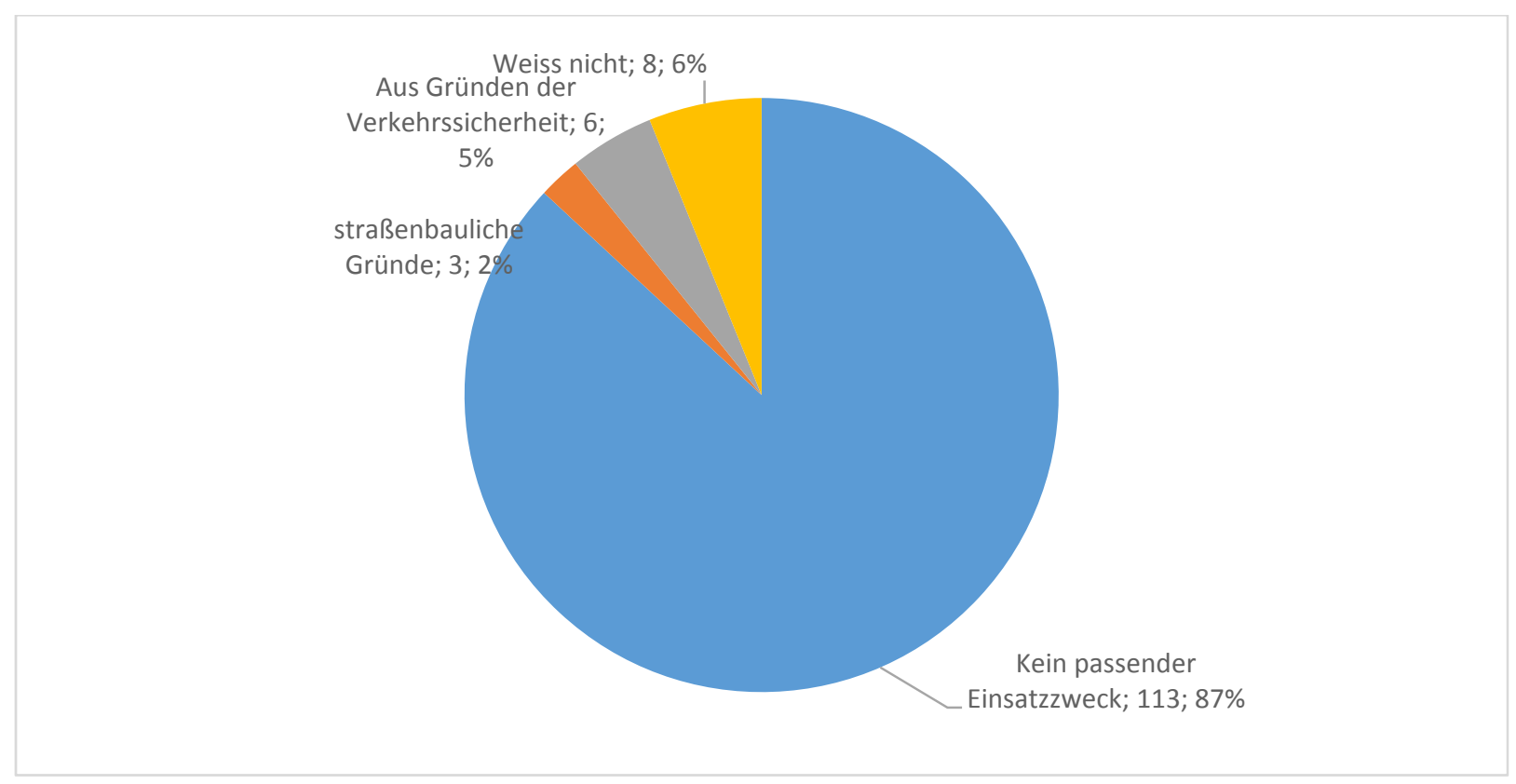

Abbildung 21: Gründe gegen die Ausweisung von Fahrradstraßen ( $n=130$ Kommunen)

Bemerkenswert und grundsätzlich positiv ist, dass als Grund gegen weitere Fahrradstraßen Bedenken im Hinblick auf die Verkehrssicherheit kaum vorhanden sind. Nur sechs kleinere Kommunen (5\%) gaben dieses als Begründung an. Auch straßenbauliche Gründe spielen mit drei Nennungen (2\%) nur eine untergeordnete Rolle. Der Hauptgrund mit insgesamt $87 \%$ ist, dass kein passender Einsatzzweck für das Infrastrukturelement Fahrradstraße gesehen wird. Dafür kann es grundsätzlich zwei mögliche Erklärungen geben: Entweder es besteht im Kontext der kommunalen Radverkehrsplanung fachlicher Informationsbedarf über den Sinn und Einsatzzweck von Fahrradstraßen oder die Mindestbedingungen für den Einsatz von Fahrradstraßen (Radverkehrsmenge etc.) werden nicht erfüllt. Hier besteht weiterer Untersuchungsbedarf.

Darüber hinaus wurde in Phase I erhoben, ob in den Kommunen die Ausweisung einer Fahrradstraße schon einmal zurückgenommen wurde: Letzteres war nur in fünf Städten der Fall. Dabei waren aber in keinem Fall Verkehrssicherheitsgründe ausschlaggebend.

Auch eine Vorher-Nachher-Untersuchung wird im Zusammenhang mit der Einrichtung von Fahrradstraßen offenbar nur selten vorgenommen. Lediglich 12 Kommunen (11 \% der Kommunen mit Fahrradstraßen) gaben an, im Rahmen der Einrichtung von Fahrradstraßen grundsätzlich solche Untersuchungen durchgeführt zu haben.

\section{Wahrnehmung von Fahrradstraßen}

Im Hinblick auf eine Bewertung der Verkehrssicherheit wurde in Phase II die Wahrnehmung der Fahrradstraßen erfasst. Zum einen wurde bei den Kommunen die subjektive Einschätzung der Antwortenden zur Verkehrssicherheit der einzelnen Fahrradstraßen erfragt, zum anderen wurde nachgefragt, ob es Beschwerden aus der Bevölkerung zu Fahrradstraßen gibt. 


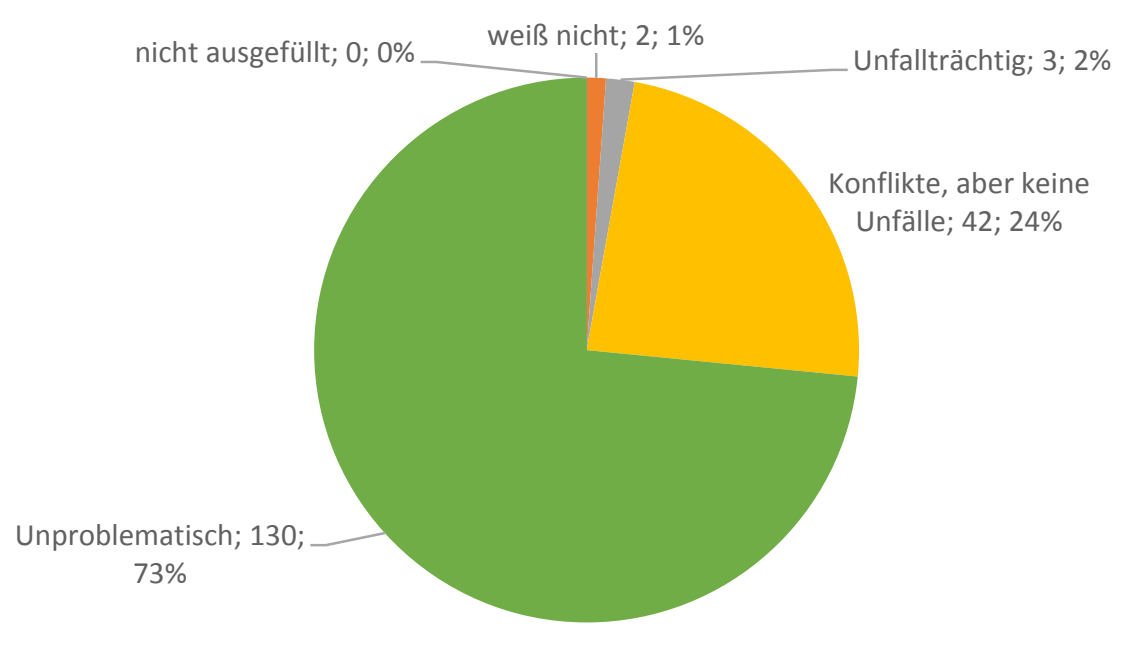

Abbildung 22: Einschätzung der Verkehrssicherheit auf den einzelnen Fahrradstraßen $(n=177)$

Wie Abbildung 22 zeigt, werden fast Dreiviertel aller Fahrradstraßen von den Kommunen als unproblematisch angesehen. Besonders positiv ist herauszuheben, dass nur $2 \%$ der Fahrradstraßen als unfallträchtig angesehen werden.

Bei den Beschwerden aus der Bevölkerung wurden die freien Textantworten kategorisiert. Als relevant für die Verkehrssicherheit der Fahrradstraße wurden Beschwerden über:

- zu hohe Kfz-Geschwindigkeit oder auch Radverkehrs-Geschwindigkeiten,

- zu hohe Verkehrsbelastungen (ohne nähere Spezifizierung),

- Fehlverhalten des Kfz-Verkehrs wie das Befahren der Fahrradstraße in falscher Richtung,

- Konflikte mit ruhendem Verkehr etc.

genannt.

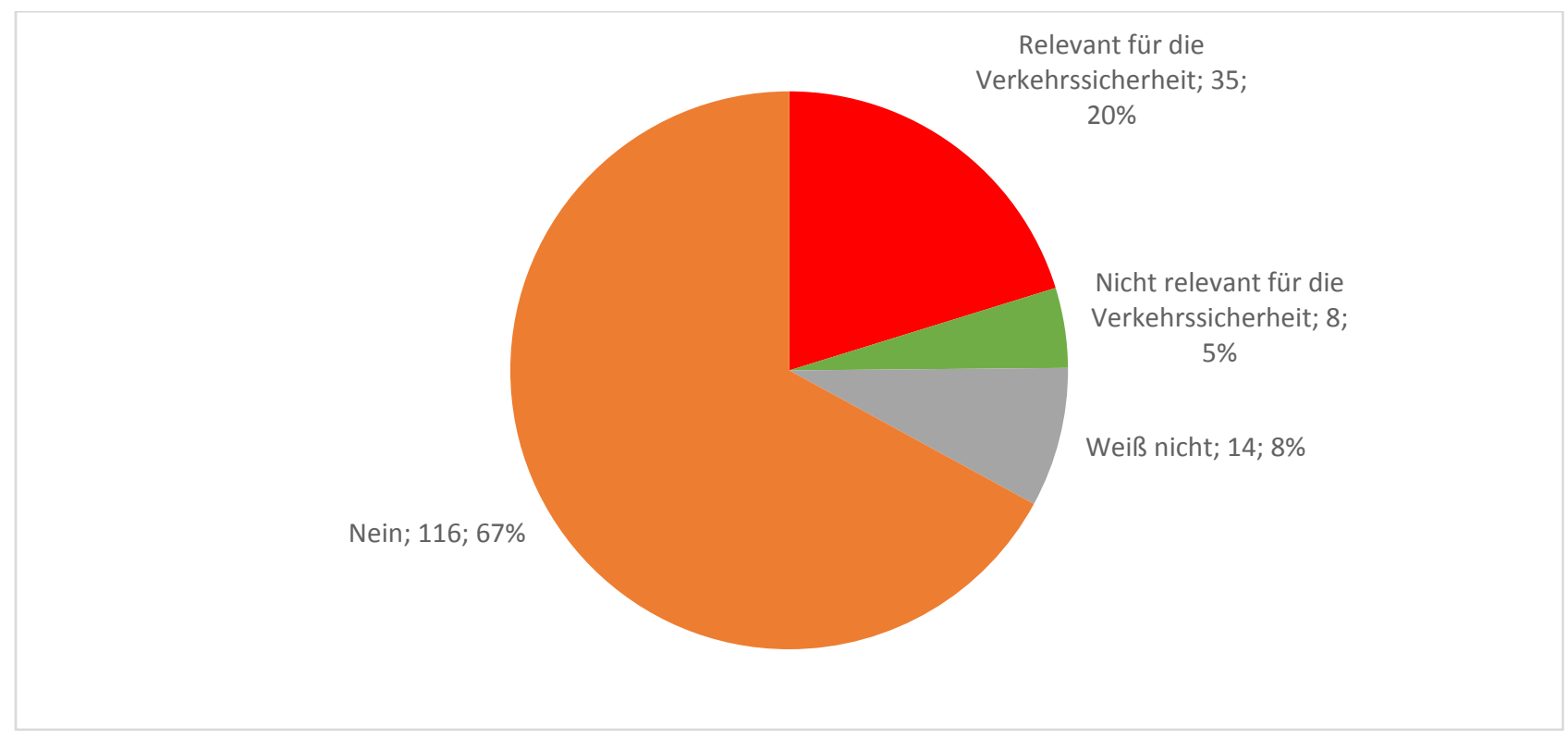

Abbildung 23: Beschwerden aus der Bevölkerung über die einzelnen Fahrradstraßen $(n=173)$ 
Es zeigt sich ein ähnlicher Befund wie bei der kommunalen Einschätzung der Fahrradstraßen: Für $67 \%$ der Straßen lagen in den Kommunen keine Beschwerden vor und weitere $5 \%$ der Beschwerden waren nicht relevant im Hinblick auf die Verkehrssicherheit. Daraus ergibt sich, dass bei $72 \%$ der Fahrradstraßen keine Beschwerden in Bezug auf die Verkehrssicherheit bei den Behörden vorlagen. Demgegenüber lagen für ein Fünftel aller Fahrradstraßen aus Phase II konkrete Beschwerden vor, die sich als relevant für die Verkehrssicherheit erwiesen (siehe Abbildung 23). Die Fragen der Verkehrssicherheit und des Unfallgeschehens wurden im Kapitel 3.3 vertieft untersucht. Darüber hinaus war dies ein wichtiger Hinweis für die Auswahl der zu beobachtenden Straßen.

\section{Gestaltung von Fahrradstraßen}

Die meisten der erhobenen Fahrradstraßen richten sich in ihrer Gestaltung nach den Empfehlungen für Radverkehrsanlagen (ERA 2010) der Forschungsgesellschaft für Straßen- und Verkehrswesen, wie sie in der Literaturauswertung dargestellt wurden. Größtenteils wird durch Markierung von Piktogrammen oder Verkehrszeichen etc. auf der Fahrbahn versucht, auf die besondere Verkehrsregelung in den Straßen aufmerksam zu machen (Abbildung 24). Es werden jedoch auch verschiedene weitergehende Maßnahmen zur Erhöhung der Verkehrssicherheit ergriffen, insbesondere die Begrenzung der Fahrbahnbreiten durch Markierungen (17\%) sowie bauliche Einengungen (15\%) und Aufpflasterungen (11\%) in Knotenpunkten. Ein knappes Sechstel der in Phase II untersuchten Fahrradstraßen (31 Straßen, $15 \%$ ) ist nur beschildert. Ein verkehrsberuhigter Ausbau oder bauliche Maßnahmen zur Erhöhung der Verkehrssicherheit sind damit eher Ausnahmen (Abbildung 24).

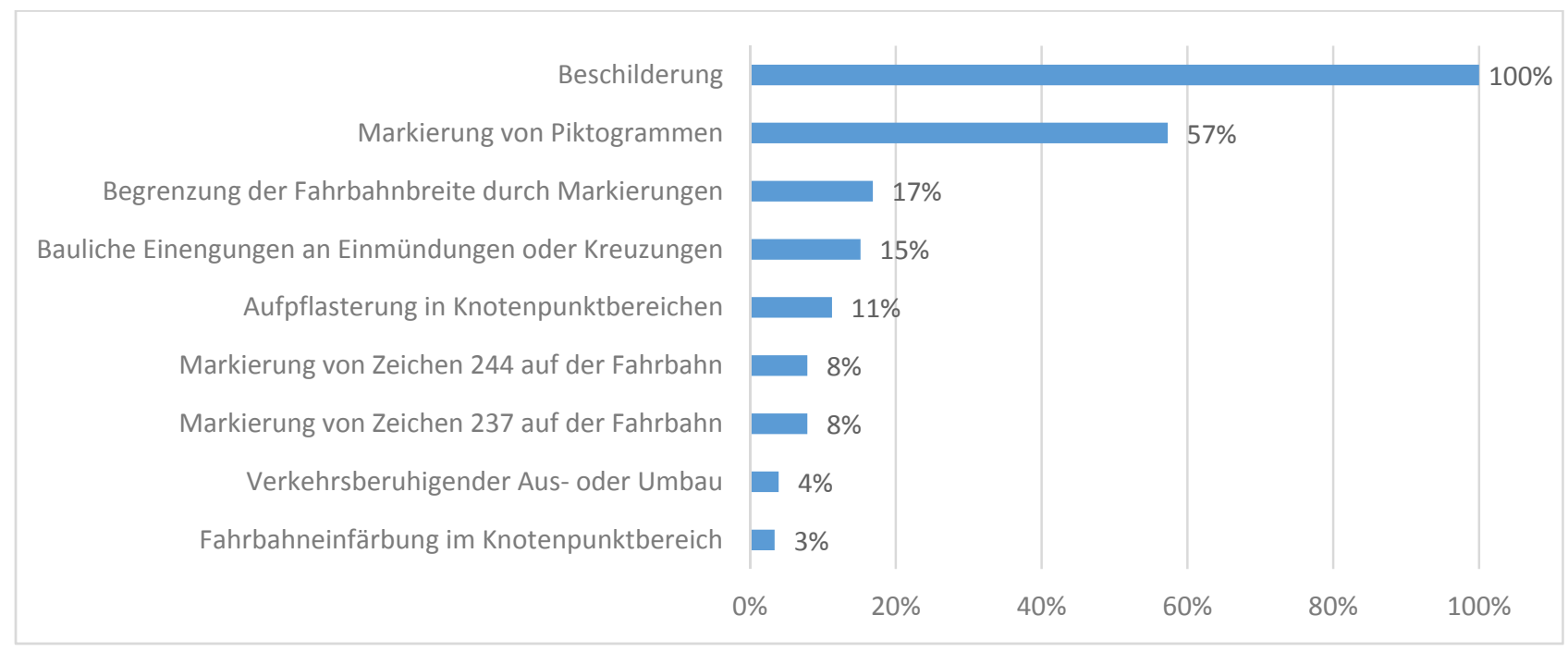

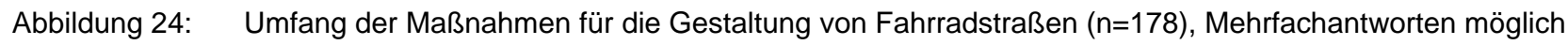
$(\Sigma=398)$

Zusätzlich wurden die Kommunen gebeten, eventuell vorhandene Gestaltungsgrundsätze für Fahrradstraßen einzureichen. Im Ergebnis wurden von drei der 110 Kommunen, die Fahrradstraßen in der Befragungsphase II genannt hatten, Gestaltungsgrundsätze vorgelegt. Dabei handelte es sich jeweils um Vorgaben zur Markierung auf der Strecke bzw. im Knotenpunkt.

Der Einfluss der Gestaltung von Fahrradstraßen auf die Verkehrssicherheit wurde im Zusammenhang mit der Unfallanalyse geprüft (siehe Kapitel 3.3). 


\section{Vorfahrtregelung an Knotenpunkten}

Die Rechts-vor-links-Regelung stellt mit $38 \%$ die häufigste vorfahrtrechtliche Regelung an den Einmündungen und Knotenpunkten von Fahrradstraßen dar. Lediglich in $28 \%$ der Fahrradstraßen wird die Fahrradstraße - mit Ausnahme von Knotenpunkten mit kreuzenden Hauptverkehrsstraßen - bevorrechtigt geführt. In $16 \%$ der Fahrradstraßen wechselt die Vorfahrtberechtigung in ihrem Verlauf, ist also nicht einheitlich geregelt. In $18 \%$ der Straßen existieren keine Einmündungen und Knotenpunkte im Verlauf der Fahrradstraße (siehe Abbildung 25).

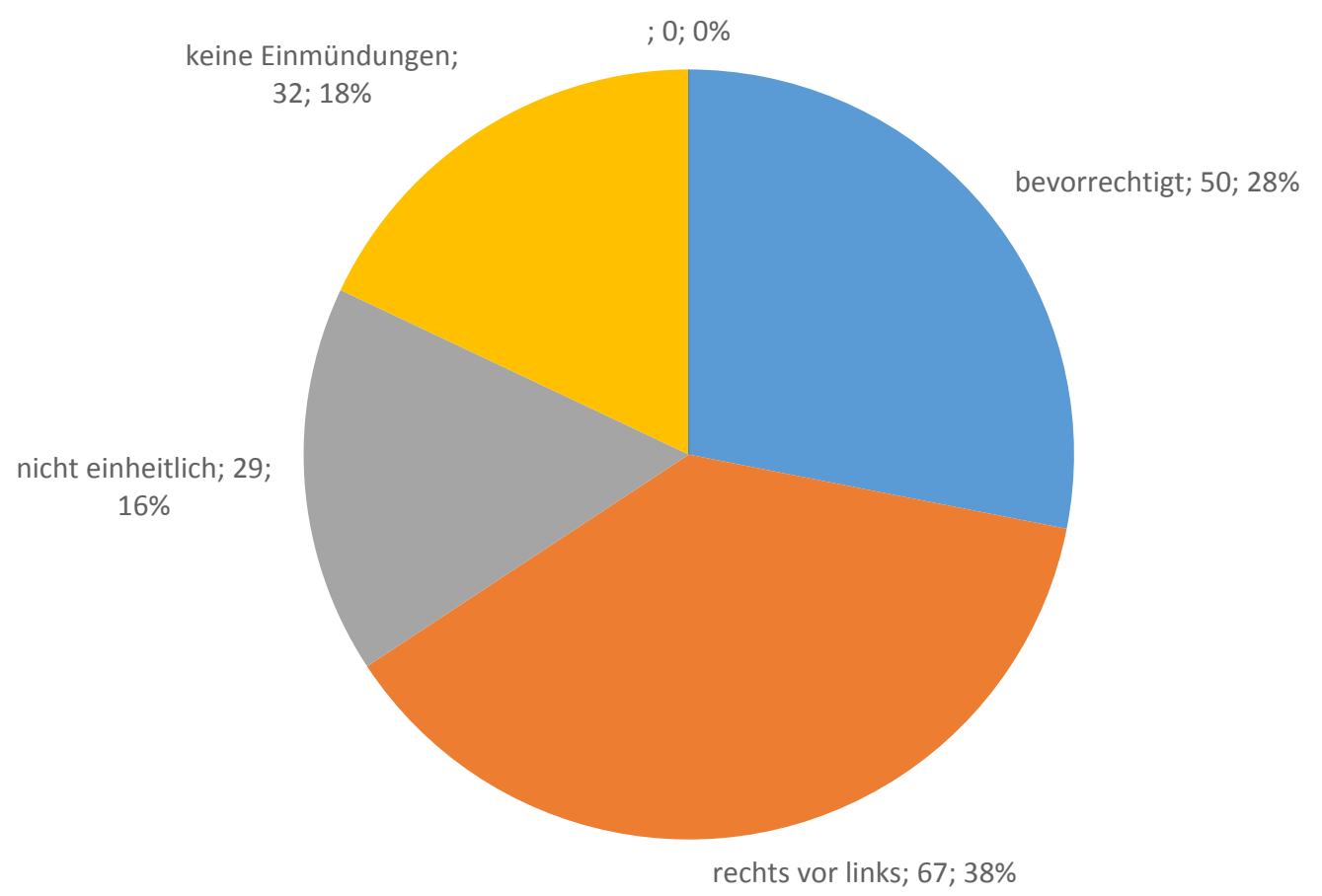

Abbildung 25: Vorfahrtregelung an den erhobenen Fahrradstraßen $(n=178)$

\section{Netzbedeutung und Funktion von Fahrradstraßen}

Wichtige Indikatoren für den Einsatz von Fahrradstraßen sind deren Bedeutung und Funktion innerhalb des Radverkehrsnetzes. Zwei Drittel der untersuchten Fahrradstraßen sind als Hauptroute Teil des Radverkehrsnetzes. Fahrradstraßen werden am zweithäufigsten (37\%) als Teile von Schulwegrouten eingesetzt (siehe Abbildung 26). Seltener werden Fahrradstraßen auf touristischen Routen und an Nebenrouten im Radverkehrsnetz eingesetzt. 


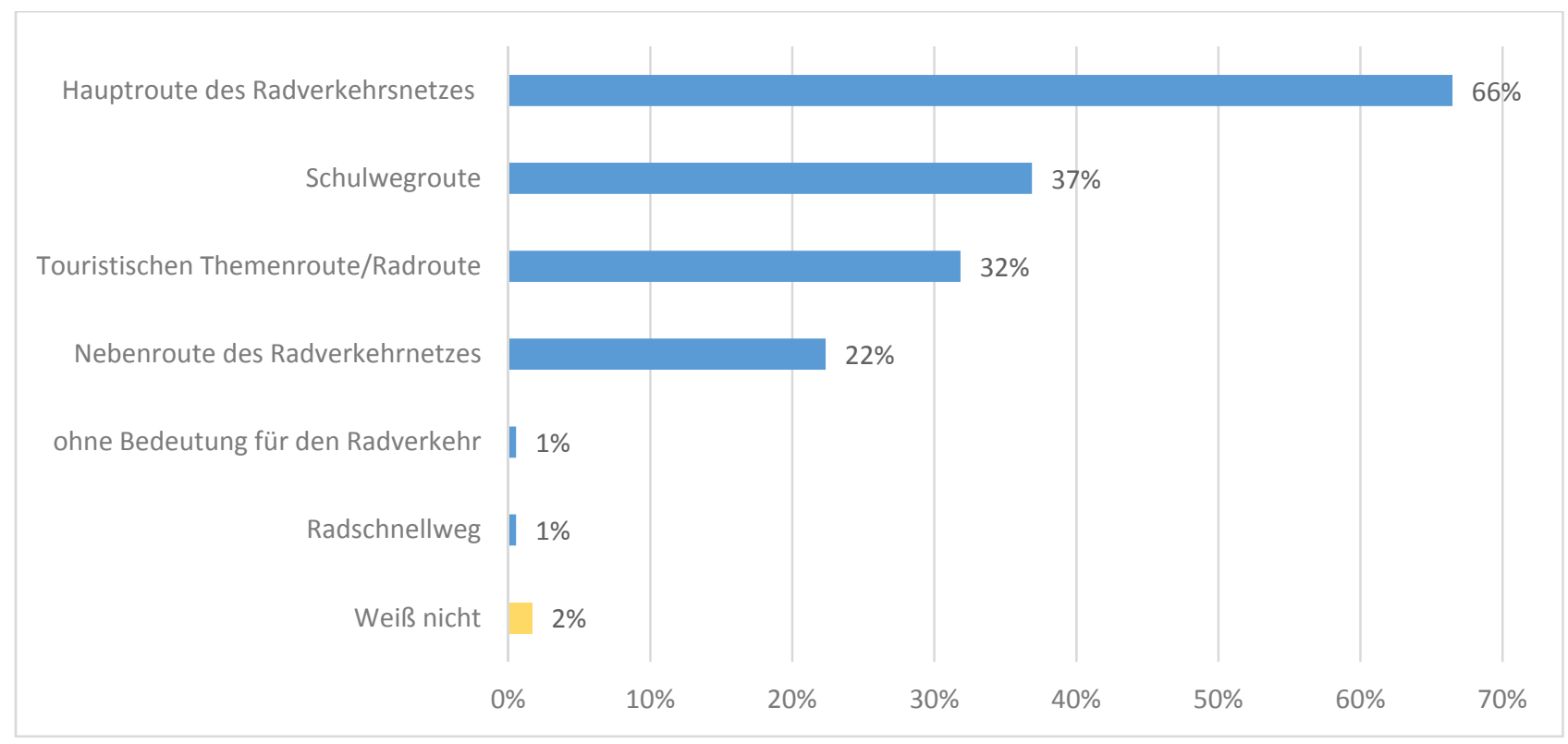

Abbildung 26: Netzbedeutung der Fahrradstraße $(n=177)$, Mehrfachnennungen möglich $(\Sigma=258)$

Im Regelfall erfüllt eine Fahrradstraße nach Angaben der Kommunen mehrere Funktionen gleichzeitig (Abbildung 27). Hauptaufgabe ist jedoch mit $88 \%$ die Verbindungsfunktion für den Radverkehr. Dies bestätigt sich beispielsweise auch in dem in diesem Zusammenhang zur Verfügung gestellten Fahrradstraßenkonzept der Stadt Bonn (STADT BONN 2012). Dort sieht man den Haupteinsatzzweck von Fahrradstraßen in der Verbindung von Ortsteilen und der Bündelung des Radverkehrs auf bestimmten Achsen.

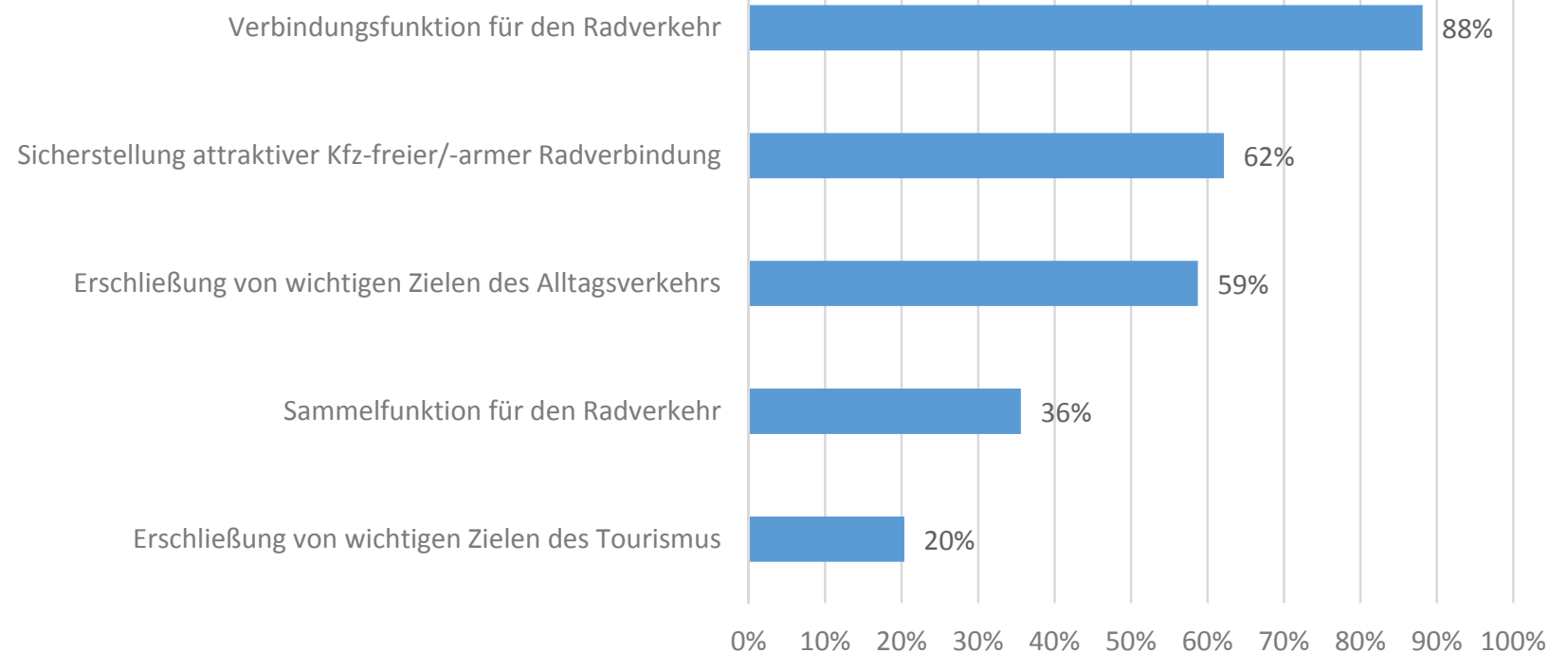

Abbildung 27: Funktion der Fahrradstraße ( $n=177)$, Mehrfachnennungen möglich $(\Sigma=469)$

Daneben sind auch die Sicherstellung Kfz- freier oder -armer Radverbindung und die Erschließung von Alltagszielen wichtige Gründe für die Einrichtung einer Fahrradstraße (59\% der Nennungen).

Betrachtet man die Netzbedeutung und die Funktionen der Fahrradstraßen gemeinsam, so zeigt sich, dass Fahrradstraßen hauptsächlich als Infrastrukturelement für den Alltagsradverkehr eingesetzt werden. Die Führung von touristischem Radverkehr oder die Erschließung von touristischen Zielen spielen nur eine untergeordnete Rolle. 


\section{Zugelassene Verkehrsarten in den Fahrradstraßen}

Nach VwV- StVO sind Fahrradstraßen grundsätzlich dem Radverkehr vorbehalten, zusätzlicher Kraftfahrzeugverkehr sollte nur ausnahmsweise zugelassen werden (vgl. Literaturauswertung Kap. 3.1). In den untersuchten Fahrradstraßen ist das Befahren durch $\mathrm{Kfz}$ dagegen in $96 \%$ der Fahrradstraßen erlaubt, in fast zwei Dritteln sogar ohne Einschränkung auf Anlieger. In nur $16 \%$ der Fahrradstraßen ist das Befahren der Kraftfahrzeuge eindeutig auf Anlieger beschränkt, zzgl. $9 \%$ der Fahrradstraßen für die „Kfz frei“ und „Anlieger frei“ angegeben wurde. Lediglich in $4 \%$ der Fahrradstraßen sind keine anderen Verkehrsarten zugelassen (Abbildung 28).

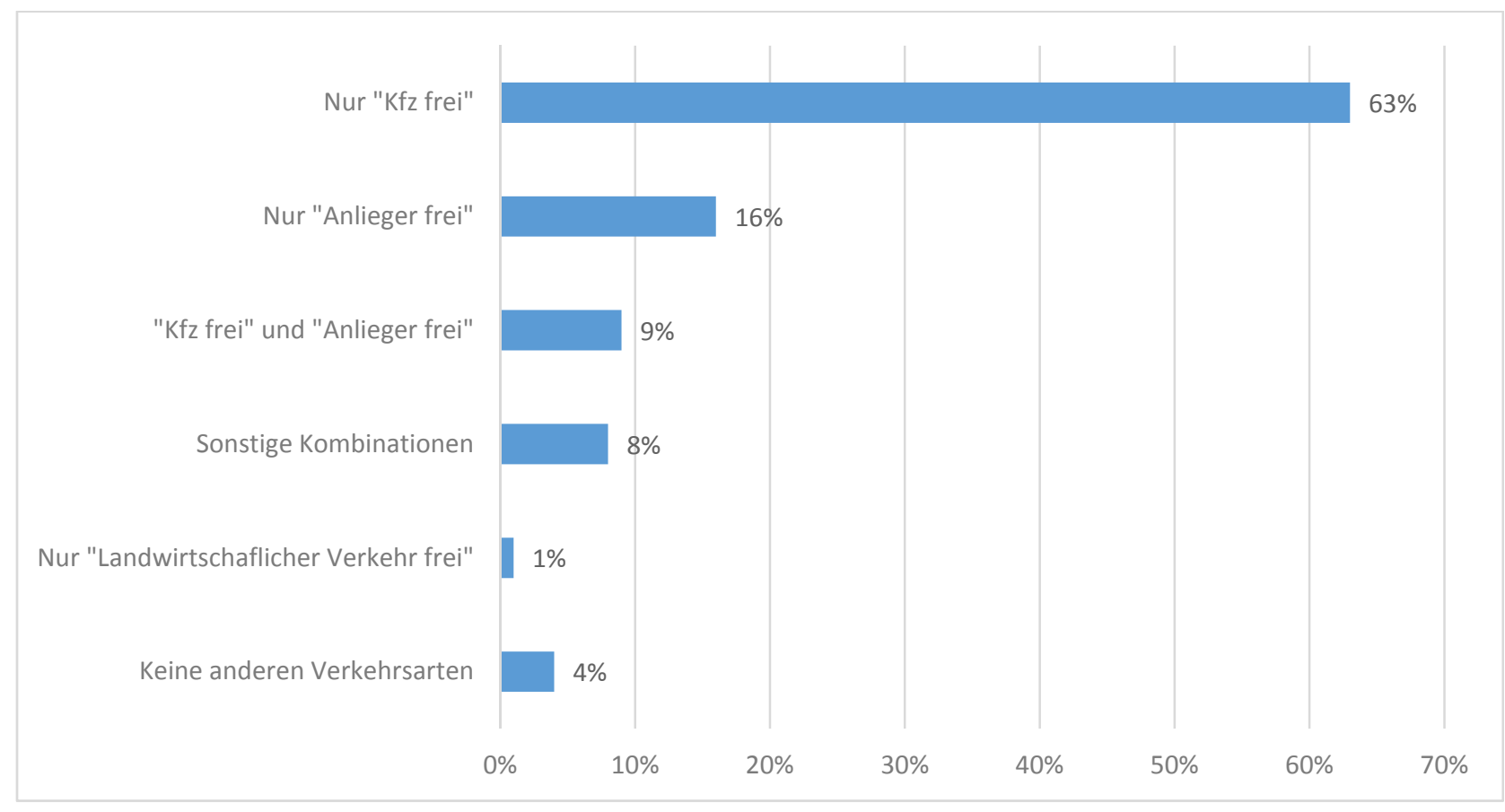

Abbildung 28: Zufahrtserlaubnis für andere Verkehrsarten in Fahrradstraßen $(n=179)$

\section{Regelung des ruhenden Kfz-Verkehrs in Fahrradstraßen}

Wie in der Literaturanalyse vorgestellt wurde, haben bereits vorangegangene Untersuchungen auf die Relevanz des Themas Parken in Bezug auf die Verkehrssicherheit in Fahrradstraßen hingewiesen (vgl. Kap. 3.1). Insofern liefern die Befragungsergebnisse weitere Hinweise für die Unfallanalyse, in der der Einfluss des ruhenden Verkehrs genauer untersuchen wird (siehe Kap. 3.3). Abbildung 29 veranschaulicht, dass in $85 \%$ der Fahrradstraßen das Parken für Kfz erlaubt ist. In gut zwei Drittel der Fahrradstraßen (68 \%) werden die Fahrzeuge auf der Fahrbahn (unmarkiert und markiert) abgestellt. Das Parken im Seitenraum (Gehweg, Parkbuchten etc.) spielt nur eine untergeordnete Rolle, ebenso wie Parken auf dem Gehweg nach Zeichen 315 StVO.

Schrägparken war in den untersuchten Fahrradstraßen nicht vertreten. 


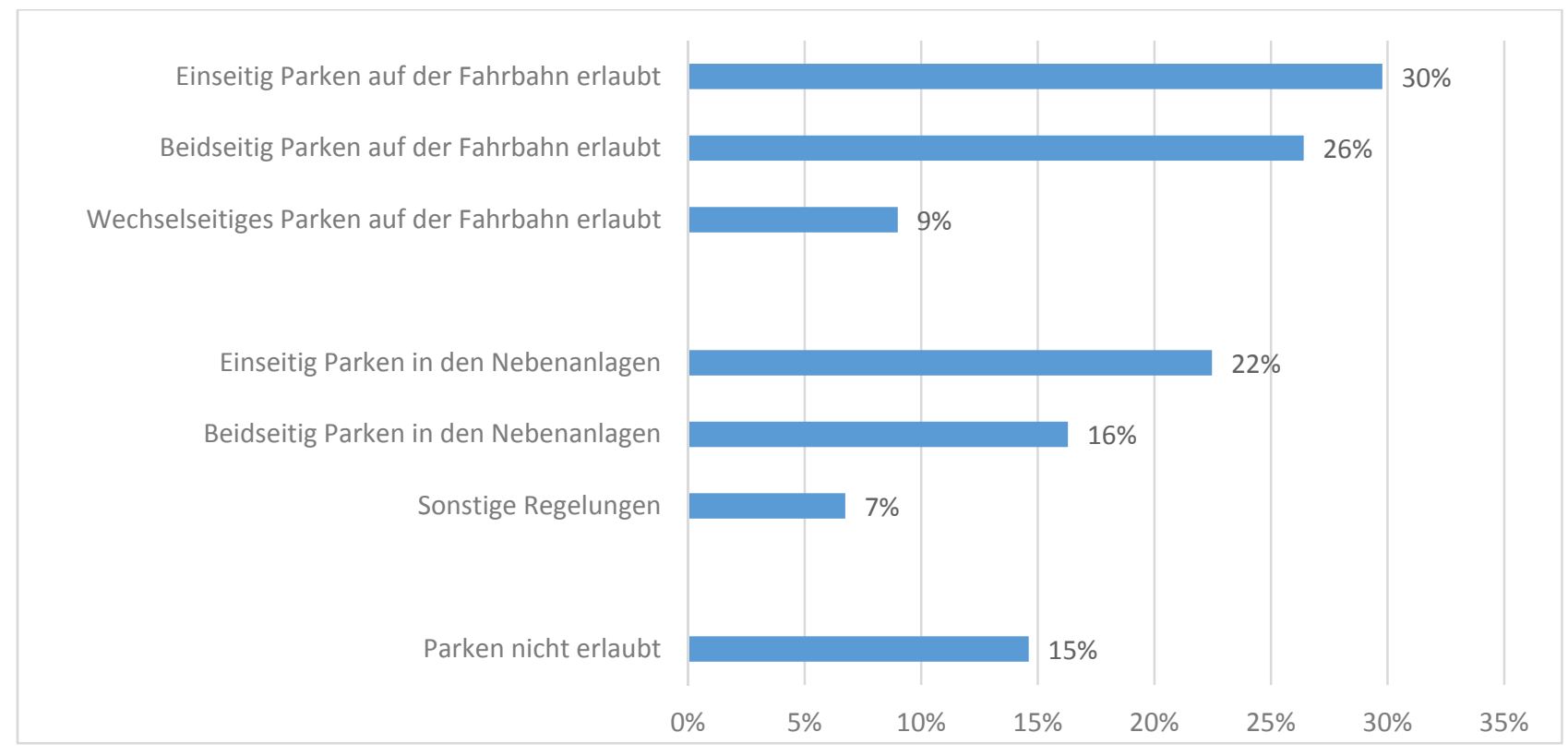

Abbildung 29: Parkregelung in den Fahrradstraßen, $n=178$, Mehrfachnennungen wegen freier Texteingabe möglich $(\Sigma=223)$

\subsubsection{Zwischenfazit aus der kommunalen Befragung}

Die Erkenntnisse aus der kommunalen Befragung haben deutlich gemacht, dass Fahrradstraßen bundesweit, jedoch nicht in allen Kommunen eingesetzt werden. Nur ein knappes Drittel der befragten Kommunen hat Fahrradstraßen ausgewiesen. $19 \%$ Kommunen planen fest mit dem Einsatz weiterer Fahrradstraßen, weitere $60 \%$ der Kommunen denken über den zukünftigen Einsatz von Fahrradstraßen zumindest nach. Bei den 130 Kommunen, die bisher keine Fahrradstraßen ausgewiesen haben und dies auch nicht beabsichtigen, liegt die Hauptbegründung (87\%) darin, dass sie keinen passenden Einsatzzweck für Fahrradstraßen in ihrer Kommune sehen. Kleinstädte und Landgemeinden unter 10.000 Einwohnern ausgenommen zeigt sich ein Zusammenhang zwischen der Kommunengröße und dem Einsatz von Fahrradstraßen, wonach der Einsatz von Fahrradstraßen mit der Stadtgröße ansteigt.

Hauptaufgabe der Fahrradstraßen ist die Verbindungsfunktion im Radverkehr (88 \%) gefolgt von einer attraktiven und Kfz-armen Radverbindung (62 \%) sowie der Erschließung von wichtigen Zielen des Alltagsverkehrs (59 \%).

Grundsätzlich gibt es eine Vielzahl von eingesetzten Gestaltungsvarianten. Es werden jedoch über die - obligatorische - Beschilderung hinaus hauptsächlich Markierungen eingesetzt. Deutlich seltener sind ein verkehrsberuhigter Ausbau oder bauliche Maßnahmen zur Erhöhung der Verkehrssicherheit. Die Kommunen folgen bei der Gestaltung von Fahrradstraßen hauptsächlich den Empfehlungen der ERA (Beschilderung, Markierung einer Kernfahrbahn für den Radverkehr, geschwindigkeitsdämpfende Maßnahmen, Markierung von Fahrradpiktogrammen an Einmündungen und Kreuzungen). Dementsprechend werden bei den befragten Kommunen auch nur in Ausnahmefällen eigene Gestaltungsrichtlinien für die Einrichtung der Fahrradstraßen zu Grunde gelegt.

Sowohl in der Verwaltung als auch bei den Verkehrsteilnehmern werden Fahrradstraßen weitgehend als positiv in Bezug auf die Verkehrssicherheit wahrgenommen. $73 \%$ der Fahrradstraßen zeigten sich in der Bewertung unproblematisch. Bei $24 \%$ der Fahrradstraßen werden Konflikte wahrgenommen, ohne dass sich dies nach Angaben der Kommunen im Unfallgeschehen abbildet. Lediglich $2 \%$ der Straßen wurden nach eigenen Angaben als 
unfallträchtig bewertet. Zu 20 \% der Fahrradstraßen liegen den befragten Planungsämtern Beschwerden bzw. Verbesserungsvorschläge aus der Bevölkerung vor.

Auf $96 \%$ der Fahrradstraßen ist Kfz-Verkehr zugelassen. Dabei überwiegt die Beschilderung (Kfz frei) mit $63 \%$ gegenüber der restriktiven Variante (Anlieger frei) mit $16 \%$.

Es konnte bundesweit weder eine einheitliche Gestaltung noch eine einheitliche Vorfahrtsregelung in Fahrradstraßen festgestellt werden.

\subsection{Unfallanalyse in Fahrradstraßen}

In den Kapiteln zu den Ergebnissen der Unfallanalyse werden die Unfalldaten zu Unfallkategorie, Unfalltyp, Unfallart, Charakteristik der Unfallstelle, Unfallbeteiligung, Unfallursachen und Unfallkonstellationen ausgewertet und die Ergebnisse dargestellt.

Es wurden weitere Parameter überprüft, die aber unauffällige Ergebnisse zeigten (siehe Kapitel 2.3.1) und nicht ausgeführt werden. Eine detaillierte Übersicht über die Zusammensetzung der Stichprobe in den einzelnen Analyseschritten wurde bereits in Kapitel 2.3.1 gegeben.

\subsubsection{Unfallschwere}

Die Auswertungen zur Unfallschwere zeigen, dass weder die Anteilswerte aller Unfälle mit Personenschaden noch die der Unfälle mit Radverkehrsbeteiligung in den untersuchten Fahrradstraßen auffällig im Vergleich zu den bundesweiten Innerortsunfällen sind. Der Anteil der Unfälle von Getöteten und Schwerverletzten ist in den Fahrradstraßen geringfügig kleiner (vgl. Abbildung 30 und Abbildung 31).

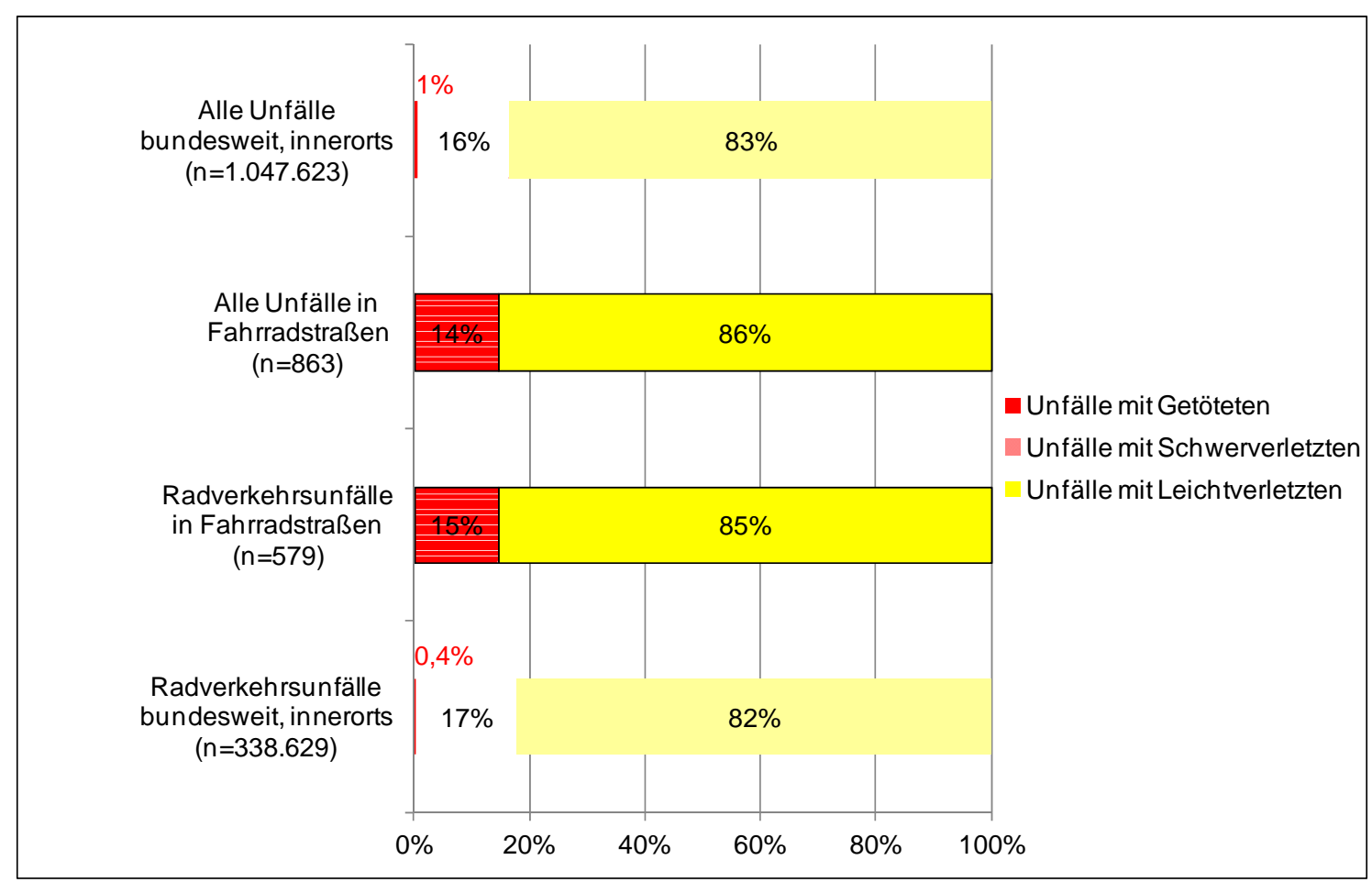

Abbildung 30: Vergleich der Unfallkategorie in Fahrradstraßen mit den bundesweiten Innerortsunfällen (U(P), 20082012) ${ }^{19}$

19 Quelle Unfälle bundesweit, innerorts: Statistisches Bundesamt (2009-2013): Fachserie 8, Reihe 7, 20082012 
Fahrradstraßen werden in der Regel auf Nebenstraßen eingerichtet. Auch ein Vergleich der Stichprobe der Radverkehrsunfälle in Fahrradstraßen mit denen in „gewöhnlichen“ Nebenstraßen ergab keine relevanten Unterschiede: Unfälle in Fahrradstraßen haben in etwa gleich schwere Unfallfolgen wie in „gewöhnlichen“ Nebenstraßen. In Bezug auf die Unfallschwere sind Fahrradstraßen für den Radverkehr demnach ähnlich wie "gewöhnliche“ Nebenstraßen, ein genereller Sicherheitsgewinn durch die Einrichtung einer Fahrradstraße lässt sich demnach nicht ableiten.

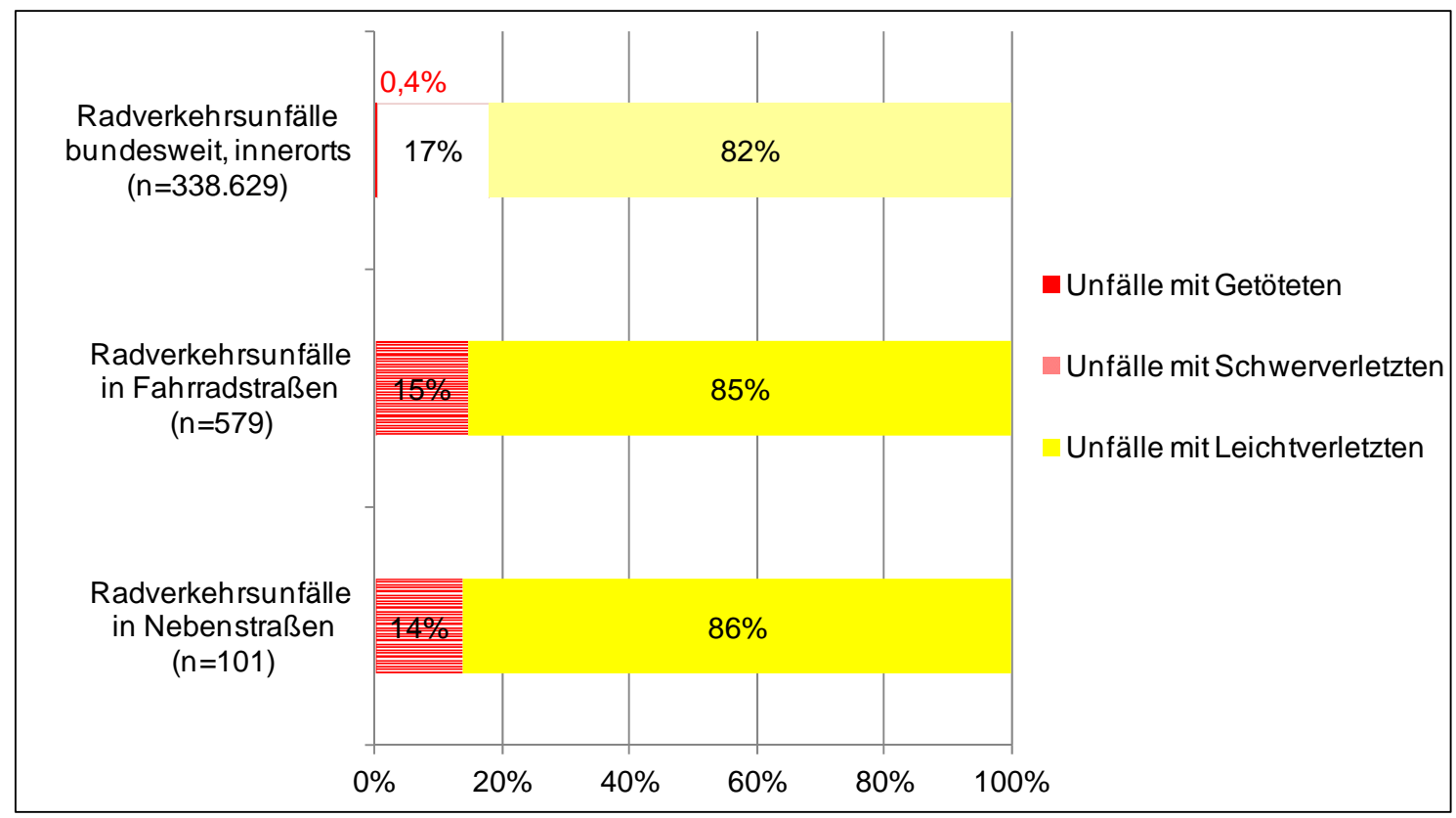

Abbildung 31: Vergleich der Unfallschwere für Radverkehrsunfälle in Fahrradstraßen (Makroanalyse) mit den bundesweiten Innerortsunfällen und den Unfällen in Nebenstraßen (U(P), 2008-2012) ${ }^{20}$

Der etwas geringere Anteil an Schwerverletzen in Fahrradstraßen und Nebenstraßen im Vergleich zu allen Innerortsstraßen ist möglicherweise auf die geringere Fahrgeschwindigkeit in beiden Straßentypen zurückzuführen. Die zusätzlich durchgeführte Auswertung zu Tempo 30-Straßen der Bundesländer Baden-Württemberg, Hessen, Sachsen und Sachsen-Anhalt (vgl. Kapitel 2.3.1) ergab, dass der Anteil der Schwerverletzten in Fahrradstraßen (15\%) und den vergleichsweise untersuchten Nebenstraßen (14\%) unter dem in Tempo 30-Straßen (20 \%) liegt.

Bei einem Vergleich aller Radverkehrsunfälle in Fahrradstraßen mit denen der für die Untersuchung relevanten Radverkehrsunfälle in Fahrradstraßen (vgl. Kapitel 2.3.1) sinkt der Anteil der Schwerverletzten geringfügig weiter (siehe Abbildung 32).

20 Quelle Unfälle bundesweit, innerorts: Statistisches Bundesamt (2009-2013): Fachserie 8, Reihe 7, 20082012 


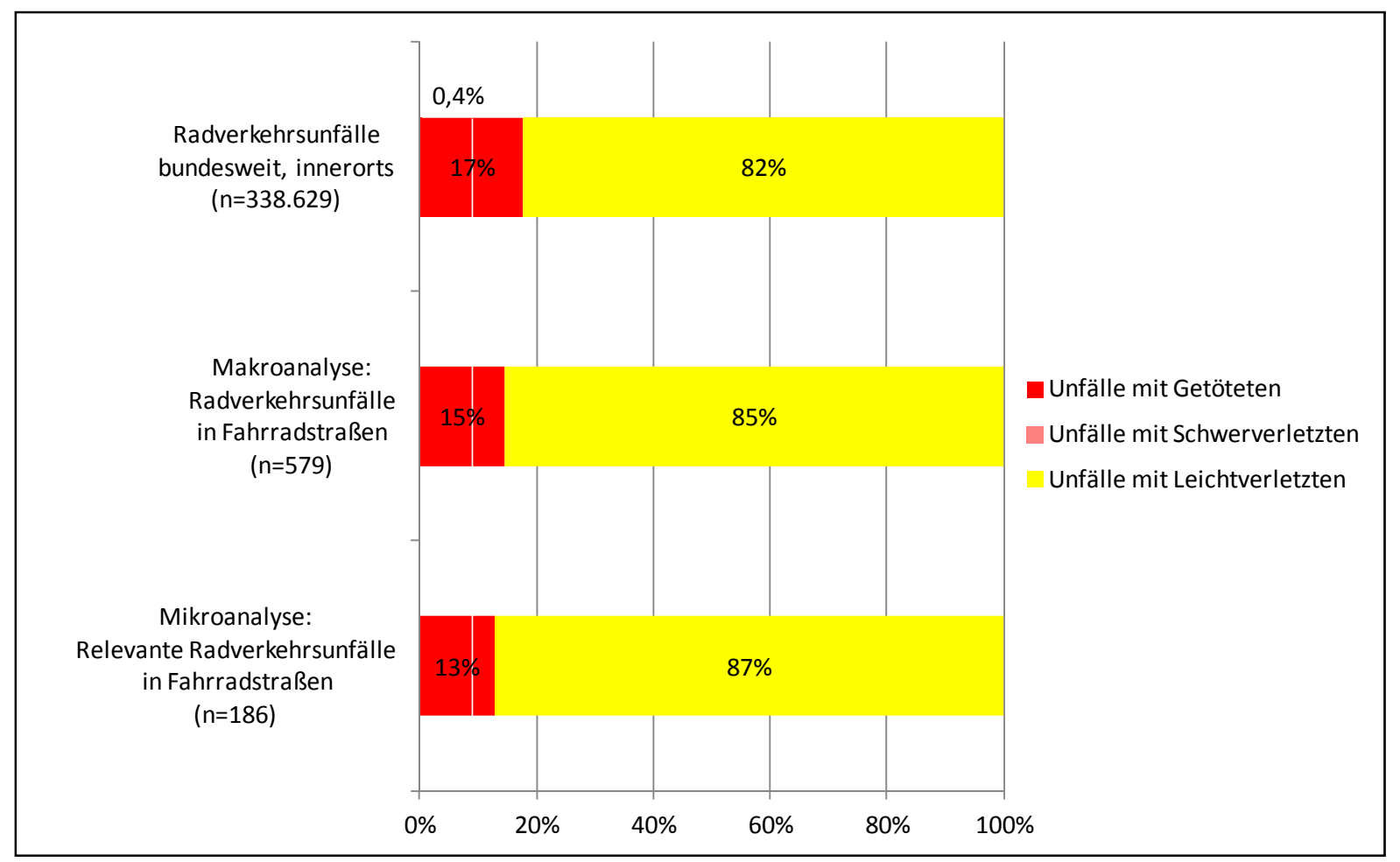

Abbildung 32: Vergleich der Unfallschwere für relevante Radverkehrsunfälle in Fahrradstraßen (Mikroanalyse) mit allen Radverkehrsunfällen in Fahrradstraßen (Makroanalyse) und den bundesweiten Radverkehrsunfällen auf Innerortsstraßen (U(P), 2008-2012) ${ }^{21}$

Dennoch bleibt auch in Fahrradstraßen ein gewisser Anteil schwerverletzter Personen bei Unfällen erhalten.

\subsubsection{Unfalltyp}

Die folgende Abbildung 33 zeigt die Auswertungen zum Unfalltyp. Der häufigste Unfalltyp ist - wie bei allen innerörtlichen Unfällen - auch in Fahrradstraßen der Einbiegen-/Kreuzen-Unfall. Darüber hinaus finden in Fahrradstraßen mehr Unfälle mit ruhendem Verkehr statt - vor allem, wenn Radfahrer beteiligt sind. Abbiege-Unfälle und sonstige Unfälle werden auf Fahrradstraßen seltener polizeilich registriert als im bundesweiten innerörtlichen Durchschnitt. Hier deutet sich bereits an, dass dies auf die Fahrbahnführung des Radverkehrs zurückzuführen ist, bei der die typischen Abbiegeunfälle zwischen abbiegenden Kfz und geradeaus fahrenden Radfahrern deutlich seltener sind als bei Führungen im Seitenraum. Erhärtet wird diese Annahme durch die Auswertung der typischen Unfallkonstellationen in der Mikroanalyse (siehe Kapitel 3.3.7).

${ }^{21}$ Quelle Unfälle bundesweit, innerorts: Statistisches Bundesamt (2009-2013): Fachserie 8, Reihe 7, 20082012 


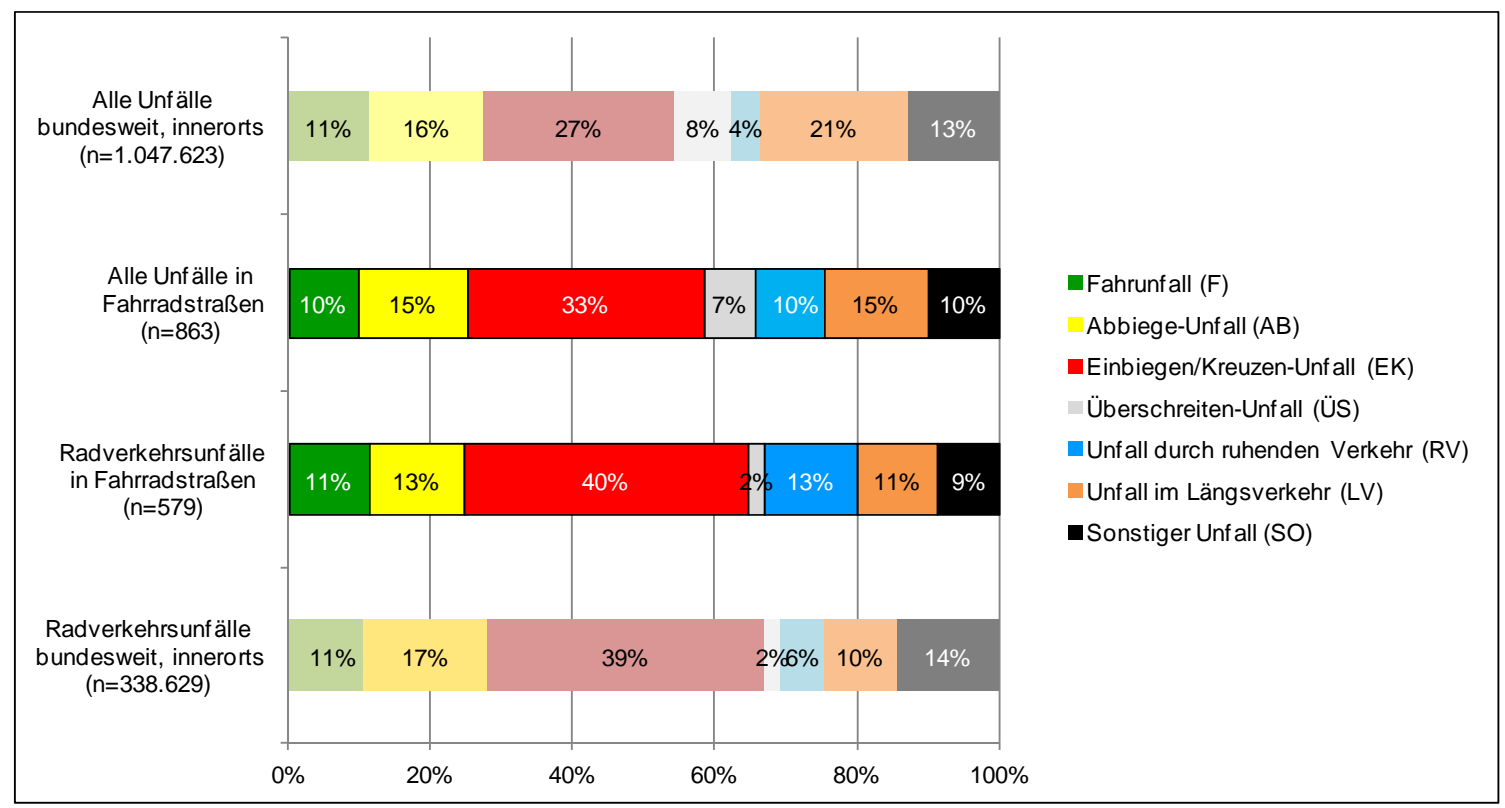

Abbildung 33: Vergleich des Unfalltyps in Fahrradstraßen mit den bundesweiten Innerortsunfällen (U(P), 2008-2012)

Vergleicht man die Radverkehrsunfälle in Fahrradstraßen mit denen auf Innerortsstraßen und denen auf Nebenstraßen, so ergibt sich folgendes Bild (siehe Abbildung 34):

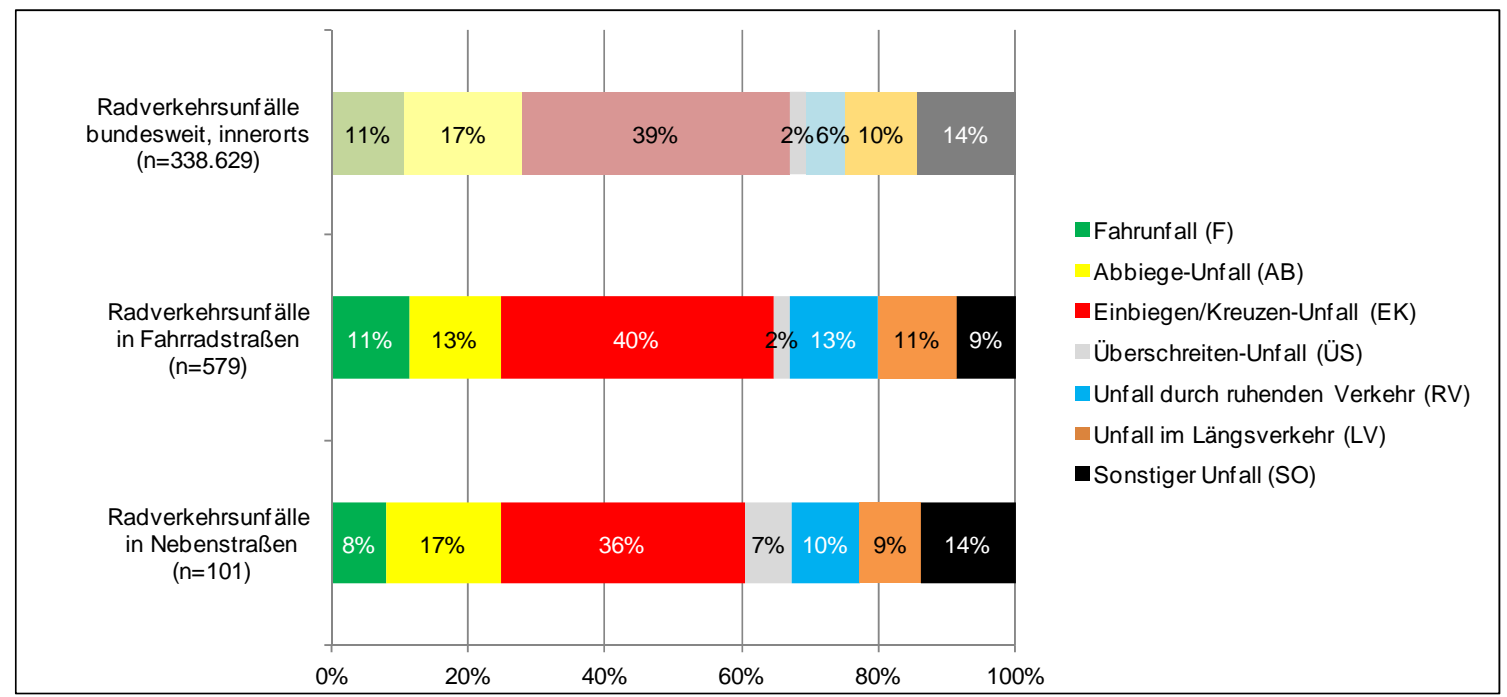

Abbildung 34: Vergleich des Unfalltyps von Radverkehrsunfällen in Fahrradstraßen mit den bundesweiten Innerortsunfällen und mit Nebenstraßen $(U(P), 2008-2012)^{23}$

In allen Unfallkollektiven bleibt der Einbiegen/Kreuzen-Unfall der häufigste Unfalltyp, in Fahrradstraßen und den untersuchten Nebenstraßen hat dieser Unfalltyp den höchsten Anteilswert. Auch die Unfälle mit ruhendem Verkehr haben in Fahrradstraßen einen erhöhten Anteilswert, auch noch einmal höher als in den Nebenstraßen. Schließlich sind in den

Quelle Unfälle bundesweit, innerorts: Statistisches Bundesamt (2009-2013): Fachserie 8, Reihe 7, 20082012

${ }^{23}$ Quelle Unfälle bundesweit, innerorts: Statistisches Bundesamt (2009-2013): Fachserie 8, Reihe 7, 20082012 
Fahrradstraßen im Vergleich zu den Nebenstraßen auch die Fahrunfälle und die Unfälle im Längsverkehr leicht höher. Deutlich geringere Anteilswerte haben dagegen Überschreiten und Sonstige Unfälle in Fahrradstraßen im Vergleich zu den Nebenstraßen. Die zusätzlich durchgeführten Auswertungen zum Unfalltyp in Tempo 30-Straßen haben keine nennenswerten Unterschiede zu den Auswertungen zum Unfalltyp in Fahrradstraßen ergeben.

Der Vergleich der Unfallschwere (Kapitel 3.3.1) und des Unfalltyps der Unfälle in Fahrradstraßen und Nebenstraßen zeigt, dass keine relevanten Unterschiede zwischen beiden Kategorien bestehen. Vielmehr zeigen sich Unterschiede zwischen den Fahrradstraßen und den Innerortsstraßen, die im Folgenden weiter ausgeführt werden.

Die erhöhten Anteilswerte in Fahrradstraßen in den Unfalltypen Einbiegen/Kreuzen-Unfall und Unfall durch ruhenden Verkehr wurden noch einmal deutlicher in der mikroskopischen Unfalluntersuchung herausgearbeitet (siehe Abbildung 35).

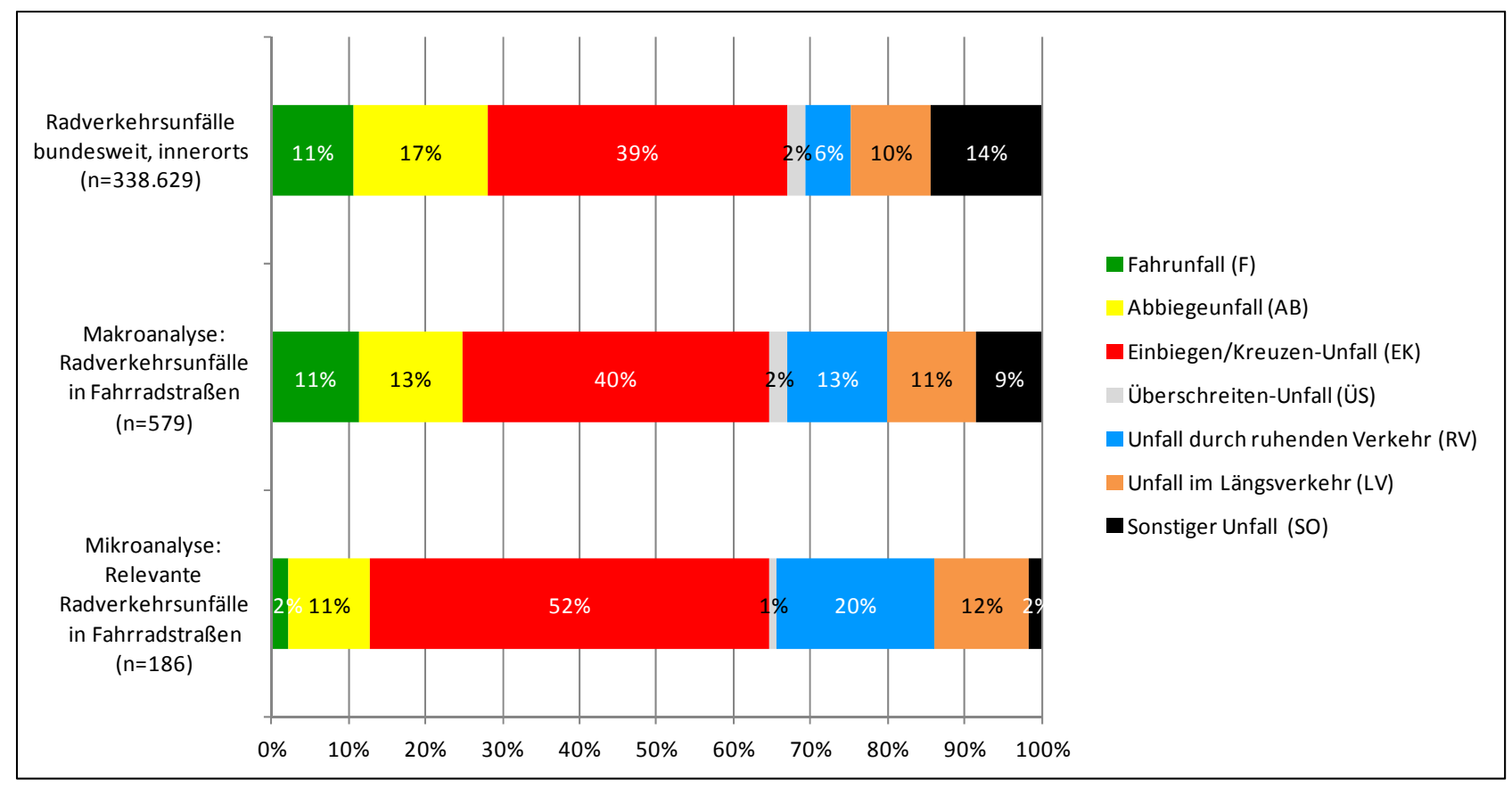

Abbildung 35: $\quad$ Vergleich des Unfalltyps für relevante Radverkehrsunfälle (Mikroanalyse) in Fahrradstraßen mit allen Radverkehrsunfällen (Makroanalyse) in Fahrradstraßen und den bundesweiten Radverkehrsunfällen auf Innerortsstraßen (U(P), 2008-2012) ${ }^{24}$

Mehr als jeder zweite relevante Radverkehrsunfall in Fahrradstraßen ist laut den Ergebnissen der Mikroanalyse ein Einbiegen/Kreuzen-Unfall und fast drei Viertel aller Unfälle in Fahrradstraßen sind Einbiegen/Kreuzen-Unfälle und Unfälle durch ruhenden Verkehr (siehe Abbildung 35).

Werden die relevanten Radverkehrsunfälle in Zwischenknotenpunkts- und Streckenunfälle unterteilt, ergibt sich ein zu erwartendes, eindeutiges Bild: Fast alle der relevanten Unfälle an Zwischenknotenpunkten in Fahrradstraßen sind Einbiegen/Kreuzen-Unfälle oder Abbiegeunfälle. Mehr als die Hälfte der relevanten Radverkehrsunfälle auf der Strecke sind Unfälle mit ruhendem Verkehr und noch einmal mehr als ein Fünftel sind Unfälle im Längsverkehr (siehe Abbildung 36).

24 Quelle Unfälle bundesweit, innerorts: Statistisches Bundesamt (2009-2013): Fachserie 8, Reihe 7, 20082012 


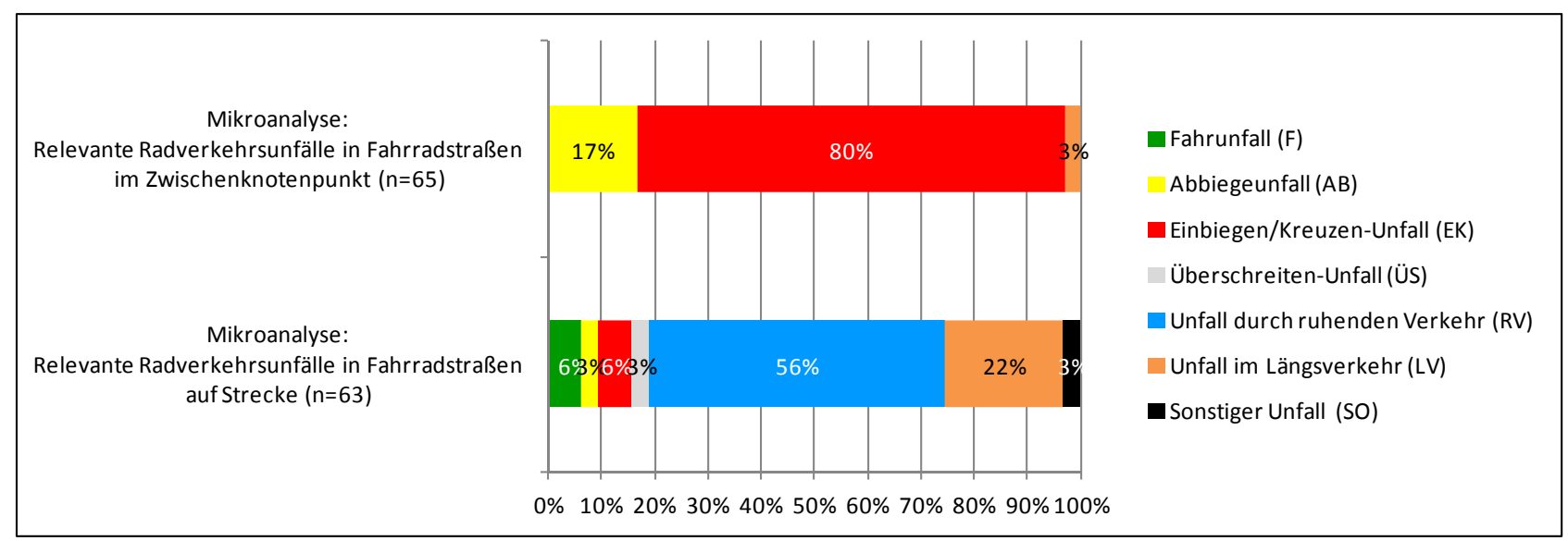

Abbildung 36: Vergleich des Unfalltyps für relevante Radverkehrsunfälle in Fahrradstraßen an Zwischenknotenpunkten mit denen auf der Strecke $(U(P), 2008-2012){ }^{25}$

\subsubsection{Unfallart}

Während der Unfalltyp den Verkehrsvorgang beziehungsweise die Konfliktsituation, aus der ein Verkehrsunfall entstanden ist, bezeichnet, macht die Unfallart Aussagen ob und wie die Unfallgegner kollidiert sind.

Die Auswertungen zur Unfallart bestätigen die Ergebnisse zum Unfalltyp: In Fahrradstraßen finden mehr Unfälle mit anfahrenden/anhaltenden/ruhenden Fahrzeugen statt als im Durchschnitt der bundesweiten innerörtlichen Unfälle mit Radverkehrsbeteiligung. Die hohe Anzahl dieser Unfallart deutet erneut auf Sicherheitsdefizite für den Radverkehr in Fahrradstraßen in Bezug auf das KfzParken hin (siehe auch Kapitel 3.3.2 zum Unfalltyp).

Die hohe Bedeutung der Unfallart Zusammenstoß mit einem einbiegenden/kreuzenden Fahrzeug bei den Unfällen mit Radverkehrsbeteiligung gibt weitere Hinweise auf Sicherheitsdefizite für den Radverkehr an den Knotenpunkten.

Im Vergleich zu allen Unfällen in Fahrradstraßen sind die Anteilswerte bei den Radverkehrsunfällen in Fahrradstraßen bei den Unfallarten „Zusammenstoß mit entgegenkommendem Fahrzeug“ und „Zusammenstoß mit seitlich in gleicher Richtung fahrendem Fahrzeug" leicht höher (siehe Abbildung 37). 


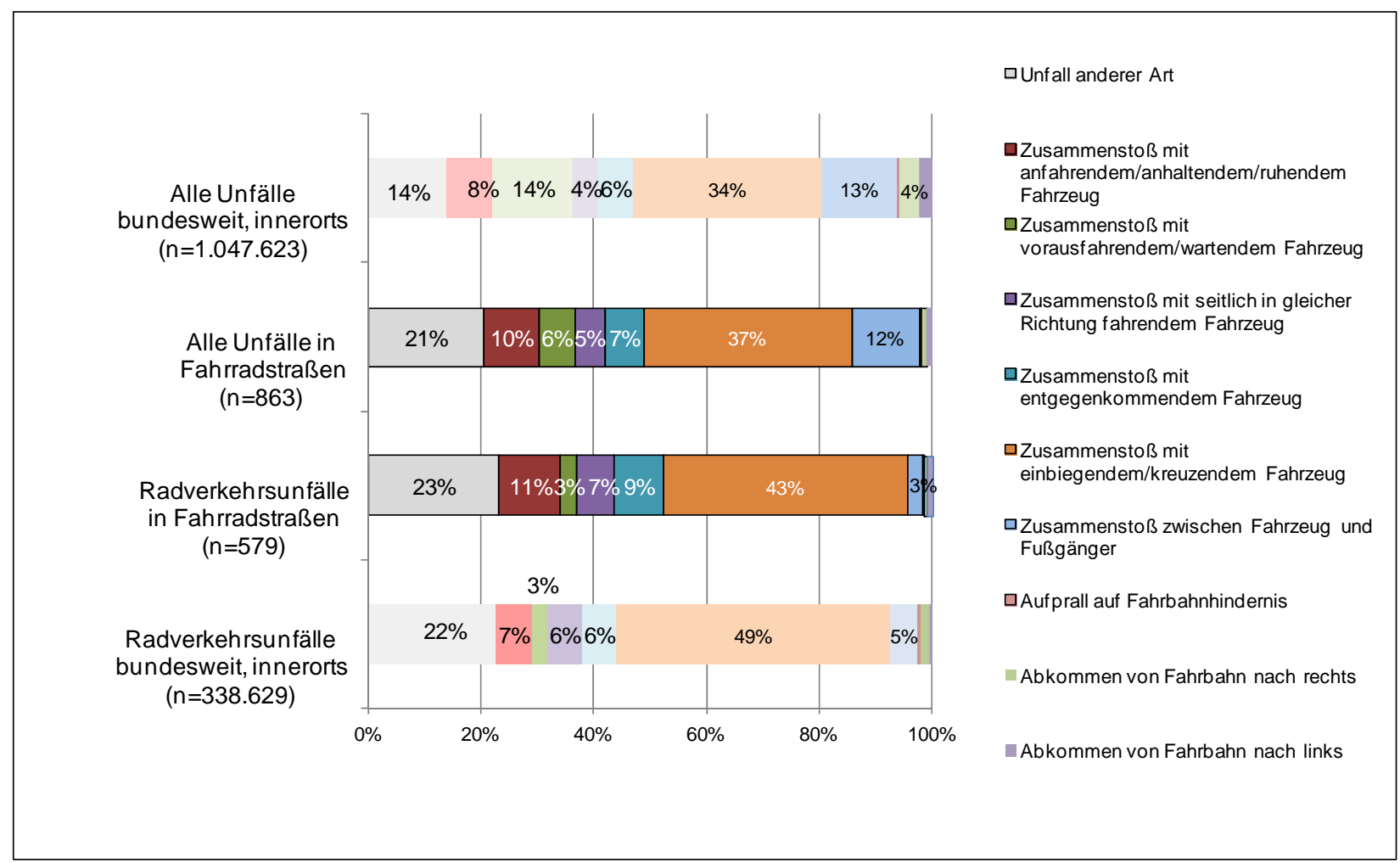

Abbildung 37: Vergleich der Unfallart in Fahrradstraßen mit den bundesweiten Innerortsunfällen (U(P), 2008-2012) 26

Bei einem Vergleich der Unfälle in Fahrradstraßen mit und ohne Radfahrerbeteiligung fällt auf, dass bei Unfällen mit Radverkehrsbeteiligung sehr häufig die Unfallart Zusammenstoß mit einbiegendem/ kreuzendem Fahrzeug registriert wird (siehe Abbildung 38). Für Unfälle ohne Radfahrerbeteiligung wird als Unfallart sehr häufig Zusammenstoß zwischen Fahrzeug und Fußgänger vermerkt. Die Unfallart wurde zusätzlich für Tempo 30-Zonen der Bundesländer Baden-Württemberg, Hessen, Sachsen und Sachsen-Anhalt ausgewertet (vgl. Kapitel 2.3.1) und auch hier konnte ein erhöhter Anteil von Unfällen mit Fußgängerbeteiligung festgestellt werden. Folglich scheint ein erhöhter Anteil von Fußgängerunfällen auch in Tempo 30-Straßen charakteristisch zu sein und nicht nur speziell in Fahrradstraßen.

26 Quelle Unfälle bundesweit, innerorts: Statistisches Bundesamt (2009-2013): Fachserie 8, Reihe 7, 20082012 und Statistisches Bundesamt, Wiesbaden 2014 


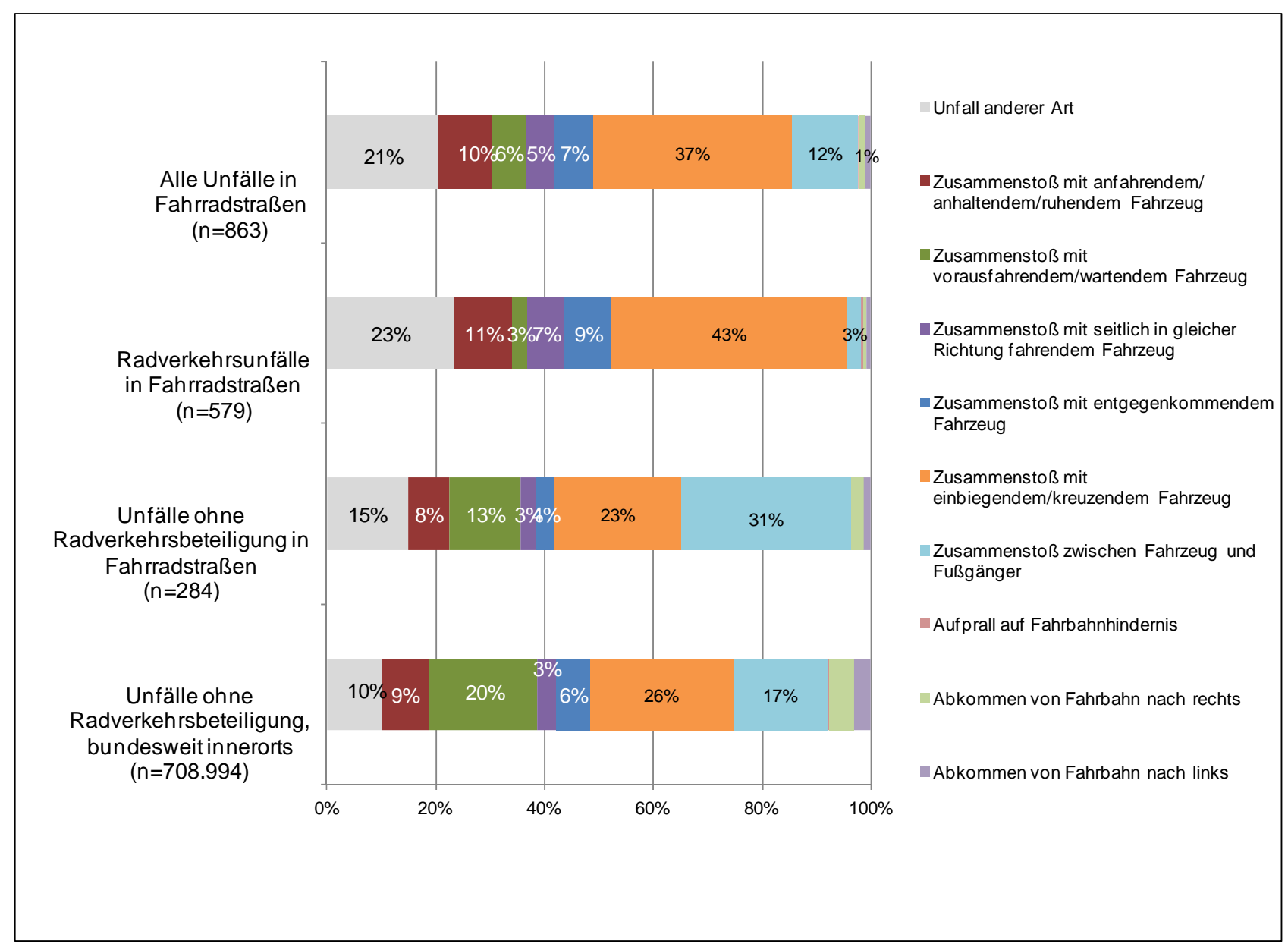

Abbildung 38: Vergleich der Unfallart in Fahrradstraßen mit und ohne Radverkehrsbeteiligung, sowie mit den bundesweiten Innerortsunfällen ohne Radverkehrsbeteiligung (U(P), 2008-2012) ${ }^{27}$

Die Betrachtung der Unfallarten bestätigt die Erkenntnisse der Untersuchung zu den Unfalltypen, dass das Kfz-Parken sowie die Gestaltung und Regelung an den Knotenpunkten besondere Bedeutung für das Unfallgeschehen haben.

27 Quelle Unfälle bundesweit, innerorts: Statistisches Bundesamt (2009-2013): Fachserie 8, Reihe 7, 20082012 und Statistisches Bundesamt, Wiesbaden 2014 


\subsubsection{Charakteristik der Unfallstelle}

In Fahrradstraßen dominieren Unfälle an Kreuzung gegenüber Unfällen an Einmündung und Grundstücksein-/-ausfahrten. Die höhere Bedeutung der Kreuzungen gegenüber Einmündungen und Grundstücksein-/-ausfahrten kann als Folge der Fahrbahnführung des Radverkehrs in Fahrradstraßen interpretiert werden, da Sicherheitsdefizite an Einmündungen und Grundstücksein/-ausfahrten eher typisch für eine Führung des Radverkehrs im Seitenraum sind. An dieser Stelle sei hierzu auf die Auswertung der Unfallkonstellationen verwiesen (siehe Kapitel 3.3.7).

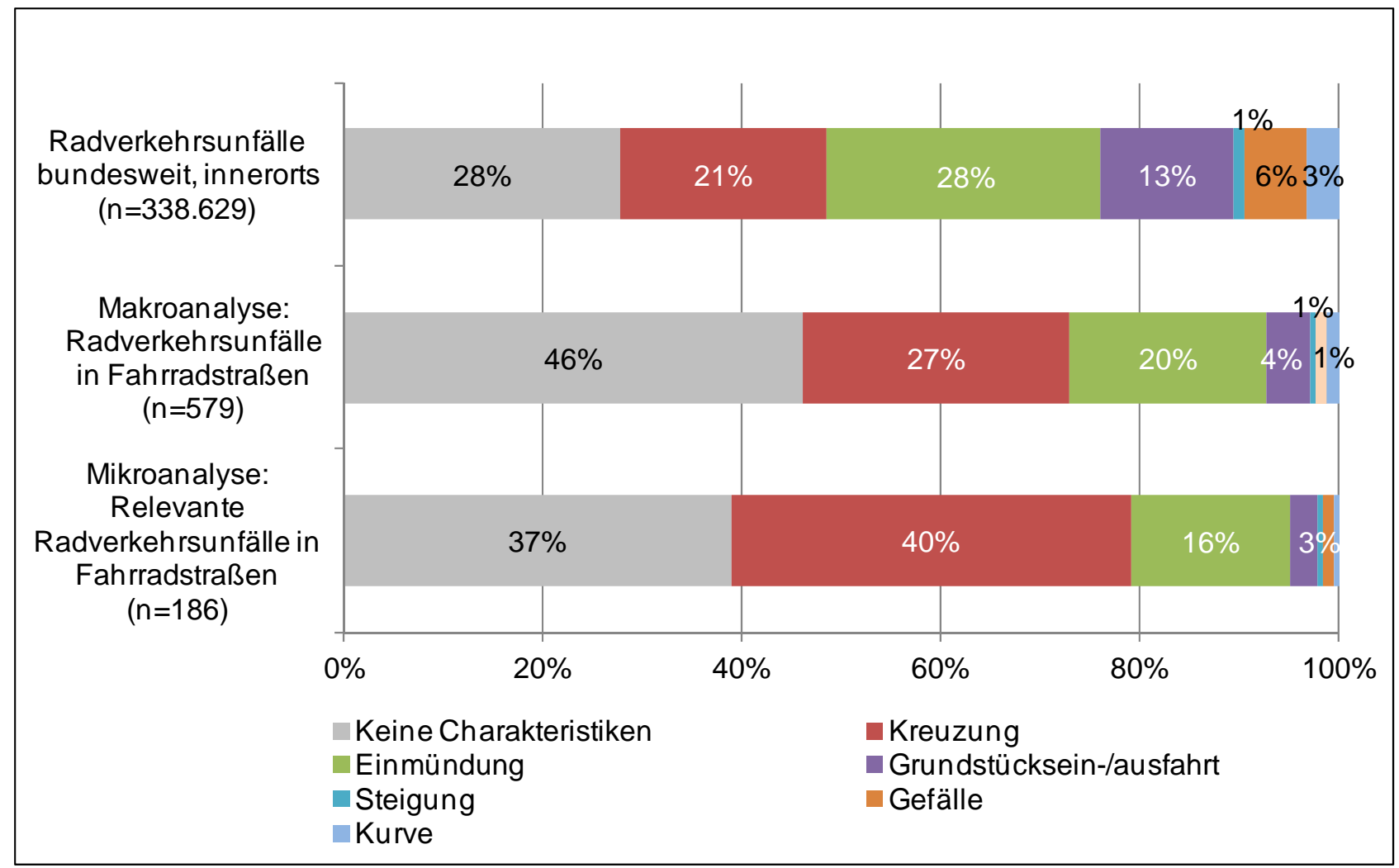

Abbildung 39: Vergleich der Charakteristik der Unfallstelle in Fahrradstraßen mit den bundesweiten Innerortsunfällen mit Radfahrerbeteiligung (U(P), 2008-2012

Die Analyse der Daten zur Charakteristik der Unfallstelle bestätigen die Ergebnisse zu Unfalltyp und Unfallart: Im Vergleich zu den Innerortsstraßen (vgl. Abbildung 39) und auch zu den zusätzlich untersuchten Tempo 30-Straßen (vgl. Kapitel 2.3.1) geschehen in Fahrradstraßen häufiger Unfälle an Kreuzungen.

\subsubsection{Unfallbeteiligte}

\section{Auswertungen zum Hauptverursacher}

Bei den Auswertungen zum Hauptverursacher der Unfälle wurde wie folgt vorgegangen: Unfälle, für die nur ein Unfallbeteiligter genannt wird, gelten als Alleinunfälle. Der erste Unfallbeteiligte wird als Hauptverursacher gewertet. Bei Unfällen mit mehreren Unfallbeteiligten sind die weiteren Unfallbeteiligten (hier die Unfallbeteiligten Zwei bis Vier) Mitverursacher, wenn auch ihnen

28 Quelle Unfälle bundesweit, innerorts: Statistisches Bundesamt (2009-2013): Fachserie 8, Reihe 7, 20082012 
Unfallursachen zugeordnet wurden. Wurden den weiteren Unfallbeteiligten keine Unfallursachen zugeordnet, gelten diese nicht als Mitverursacher, sondern als Unfallbeteiligte ohne Ursache.

$66 \%$ der Unfälle in Fahrradstraßen sind mit Radverkehrsbeteiligung. Dies ist deutlich mehr als der bundesweite innerörtliche Durchschnitt (31\%) (siehe Abbildung 40). Dies ist sehr wahrscheinlich eine Folge des höheren Anteils des Radverkehrs in Fahrradstraßen. In der Mikroanalyse wurde jedoch festgestellt, dass viele der Unfälle mit Radverkehrsbeteiligung der Makroanalyse in keinem direkten Zusammenhang mit der Infrastruktur der Fahrradstraße standen.

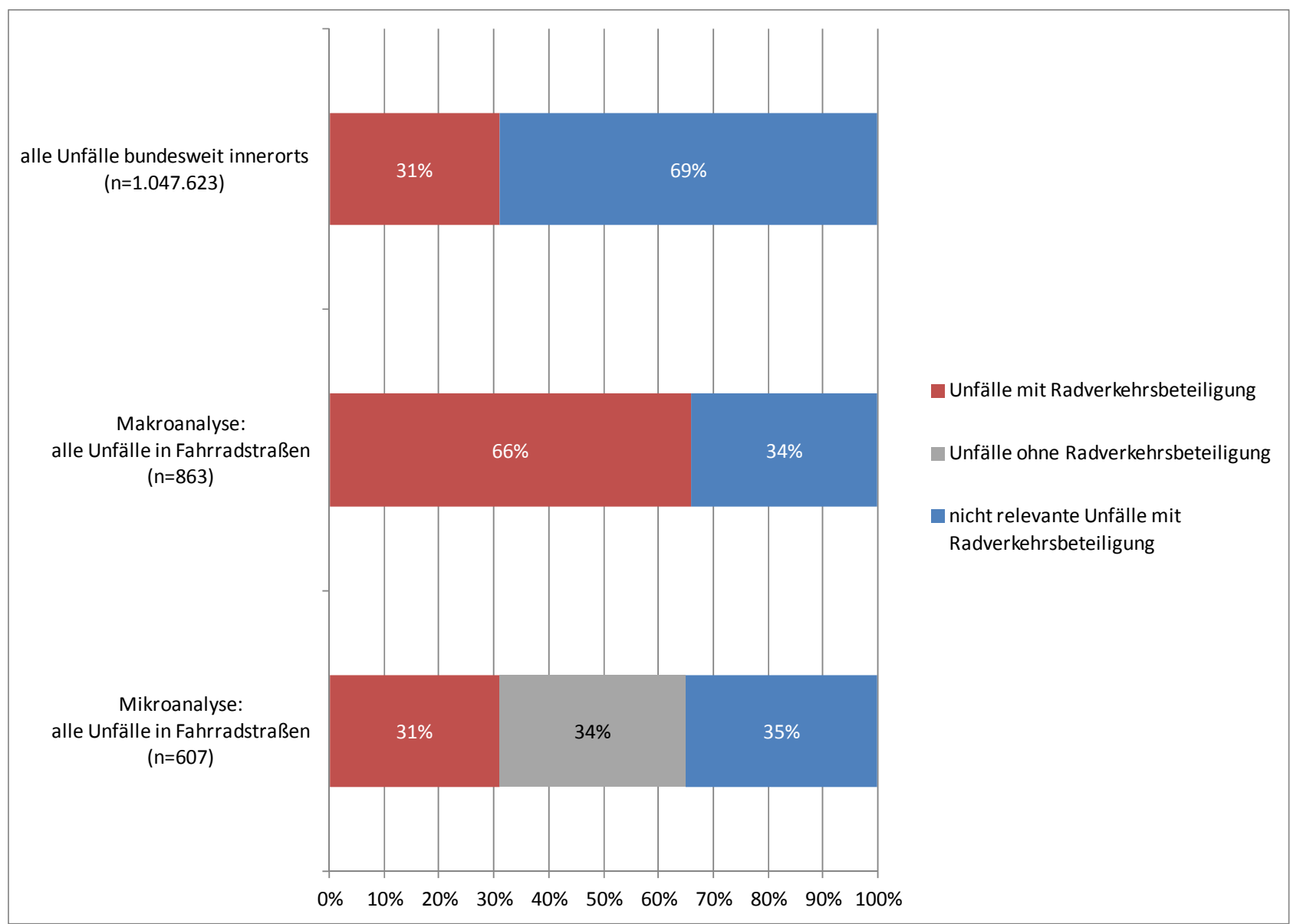

Abbildung 40: $\quad$ Anteile der Unfälle mit Radverkehrsbeteiligung in Fahrradstraßen in den Analyseschritten (U(P), 2008$2012)^{29}$

29 Quelle Unfälle bundesweit, innerorts: Statistisches Bundesamt (2009-2013): Fachserie 8, Reihe 7, 20082012 und Statistisches Bundesamt, Wiesbaden 2014 
Unfälle mit Radverkehrsbeteiligung in Fahrradstraßen weisen in der makroskopischen Analyse anteilsmäßig etwas häufiger Pkw (49 \%) als Radfahrer (44 \%) als Hauptverursacher auf (siehe Abbildung 41). Die Anteile der Hauptverursacher von Unfällen mit Radverkehrsbeteiligung in Fahrradstraßen sind damit fast identisch mit den Anteilen im bundesweiten innerörtlichen Durchschnitt aller Unfälle mit Radverkehrsbeteiligung.

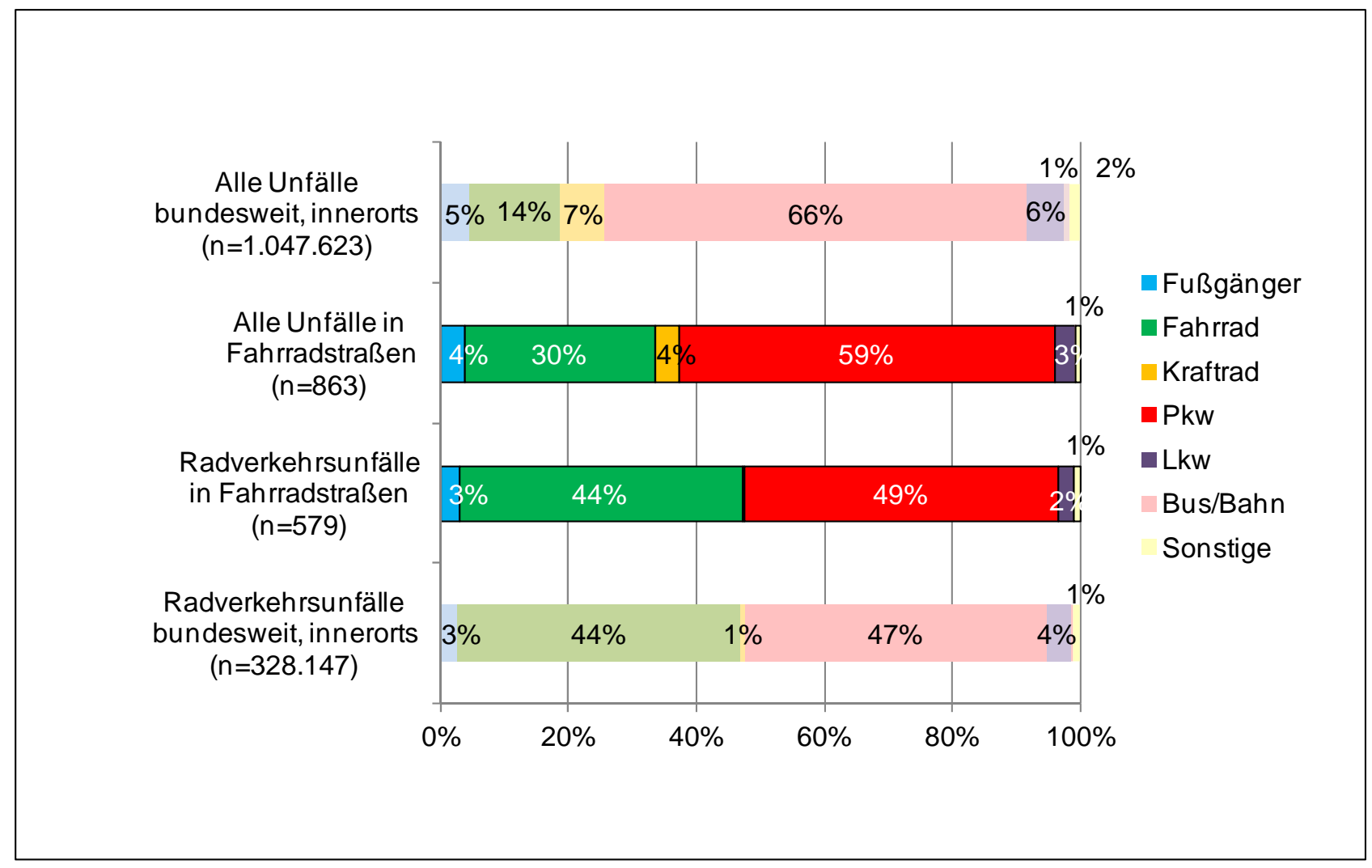

Abbildung 41: Vergleich der Hauptverursacher in Fahrradstraßen (Makroanalyse) mit denen bei bundesweiten Innerortsunfällen $(U(P), 2008-2012)^{30}$

30 Quelle Unfälle bundesweit, innerorts: Statistisches Bundesamt (2009-2013): Fachserie 8, Reihe 7, 20082012 und Statistisches Bundesamt, Wiesbaden 2014 
Bezogen auf die relevanten Radverkehrsunfälle in Fahrradstraßen in der mikroskopischen Analyse steigt der Anteil der $\mathrm{Kfz}$ als erstem Beteiligten jedoch erheblich, denn hier werden die $\mathrm{Kfz}$ deutlich häufiger als die Hauptverursacher ausgewiesen (siehe Abbildung 42).

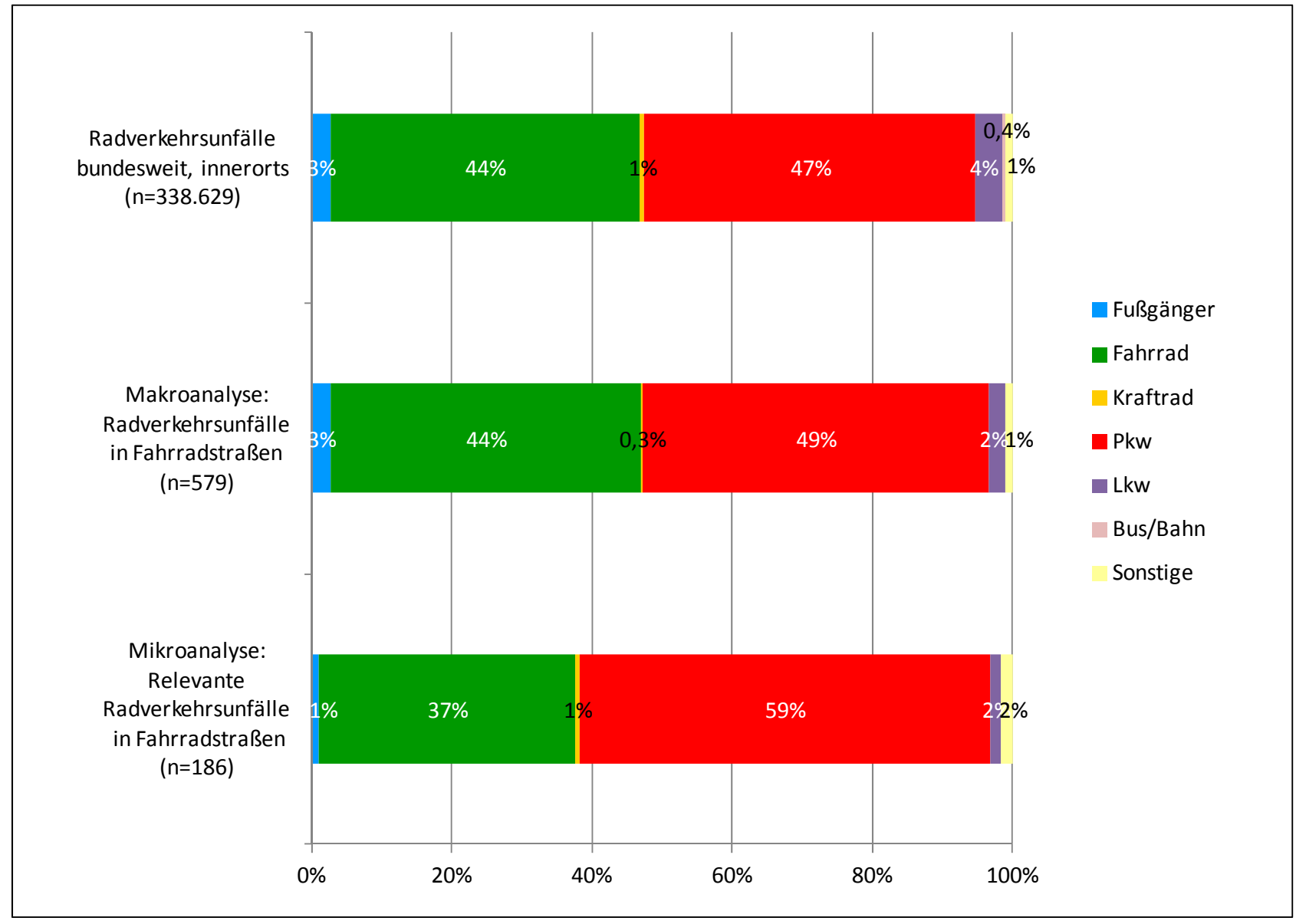

Abbildung 42: Vergleich der Hauptverursacher bei relevanten Radverkehrsunfällen in Fahrradstraßen (Mikroanalyse) mit denen bei allen Radverkehrsunfällen in Fahrradstraßen (Makroanalyse) und den bundesweiten Radverkehrsunfällen auf Innerortsstraßen (U(P), 2008-2012)

31 Quelle Unfälle bundesweit, innerorts: Statistisches Bundesamt (2009-2013): Fachserie 8, Reihe 7, 20082012 
Werden die relevanten Radverkehrsunfälle in Fahrradstraßen nach Unfallort „Strecke“ und „Zwischenknotenpunkt“ ausgewertet, so ergibt sich, dass Kfz und Kraftrad an den Knotenpunkten zu vier Fünfteln die Hauptverursacher sind. Auf der Strecke sind zu drei Vierteln die Kfz Hautverursacher; auf der Strecke nehmen aber auch die Fußgänger als Hauptverursacher eine vergleichsweise hohe Bedeutung ein (siehe Abbildung 43).

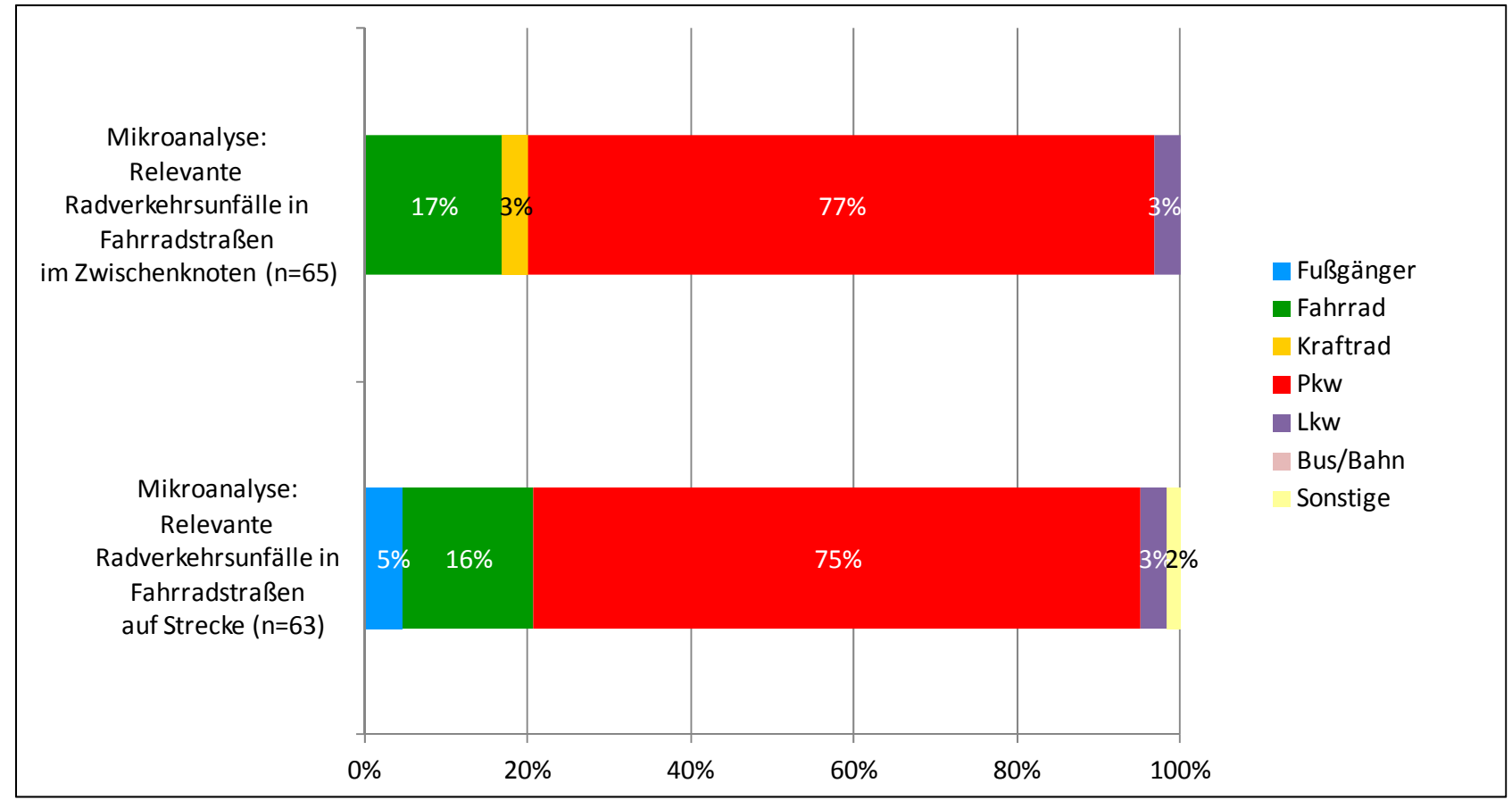

Abbildung 43: Vergleich der Hauptverursacher für relevante Radverkehrsunfälle in Fahrradstraßen (Mikroanalyse) an Zwischenknotenpunkten mit denen auf der Strecke (U(P), 2008-2012) ${ }^{32}$

32 Quelle Unfälle bundesweit, innerorts: Statistisches Bundesamt (2009-2013): Fachserie 8, Reihe 7, 20082012 


\section{Auswertungen zu Unfallgegnern}

Die Ergebnisse der Auswertung zu den ersten und zweiten Unfallbeteiligten aller Unfälle in der Makroanalyse zeigt Abbildung 44 im Detail.

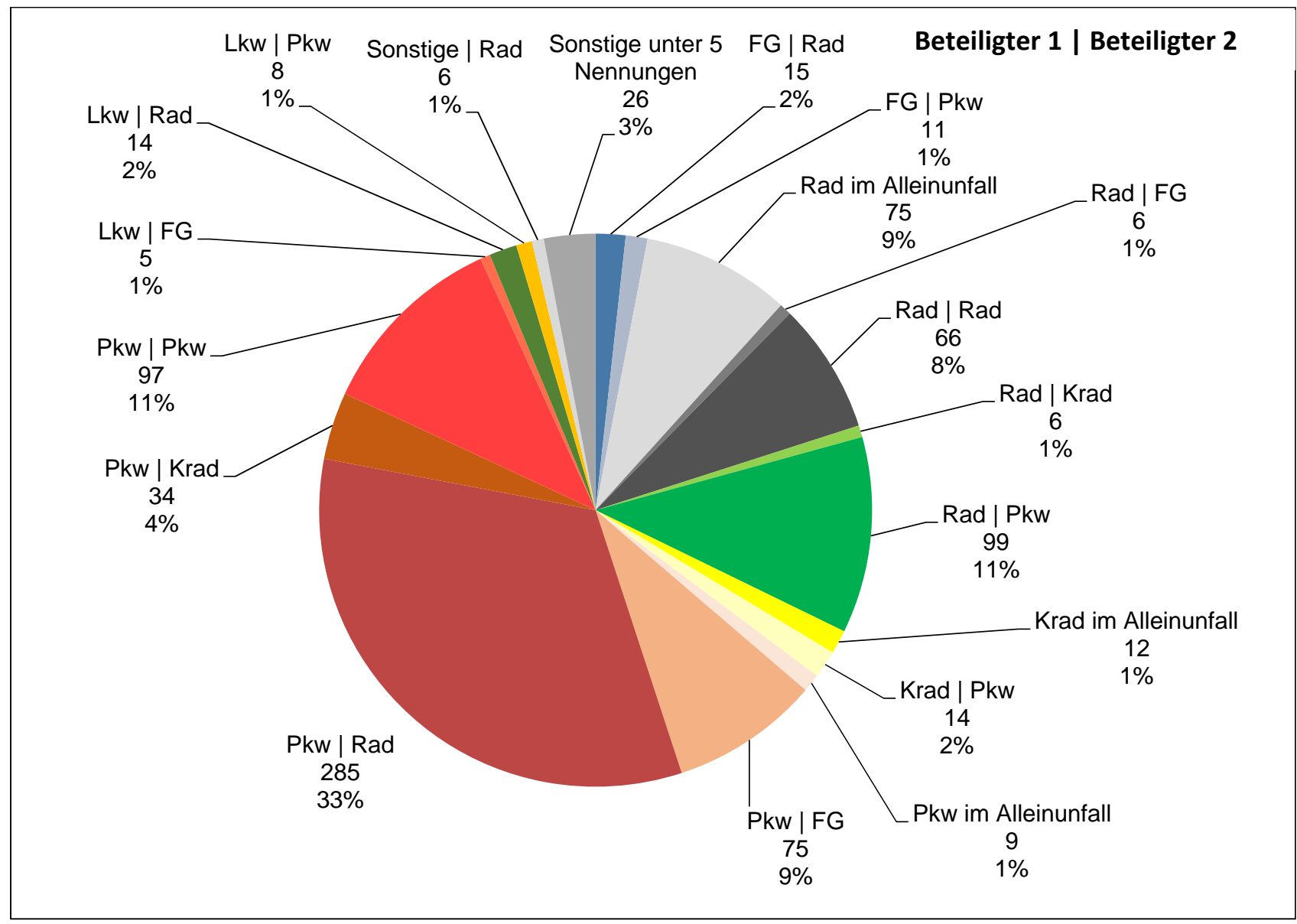

Abbildung 44: Unfallbeteiligte 1 und 2 bei allen Unfällen in Fahrradstraßen in der Makroanalyse (U(P), 2008-2012 nur Beteilige 1 und 2, $n=863$ )

Bei allen Unfällen in Fahrradstraßen, die ohne Radbeteiligung stattfinden, sind am häufigsten die Pkw | Pkw- Unfälle (11\%) und die Pkw | Fußgänger (FG)-Unfälle (9\%).

Werden bei den Unfällen mit Radverkehrsbeteiligung die Unfallgegner zusammen gefasst, so ergibt sich folgendes Bild (siehe Abbildung 45). 


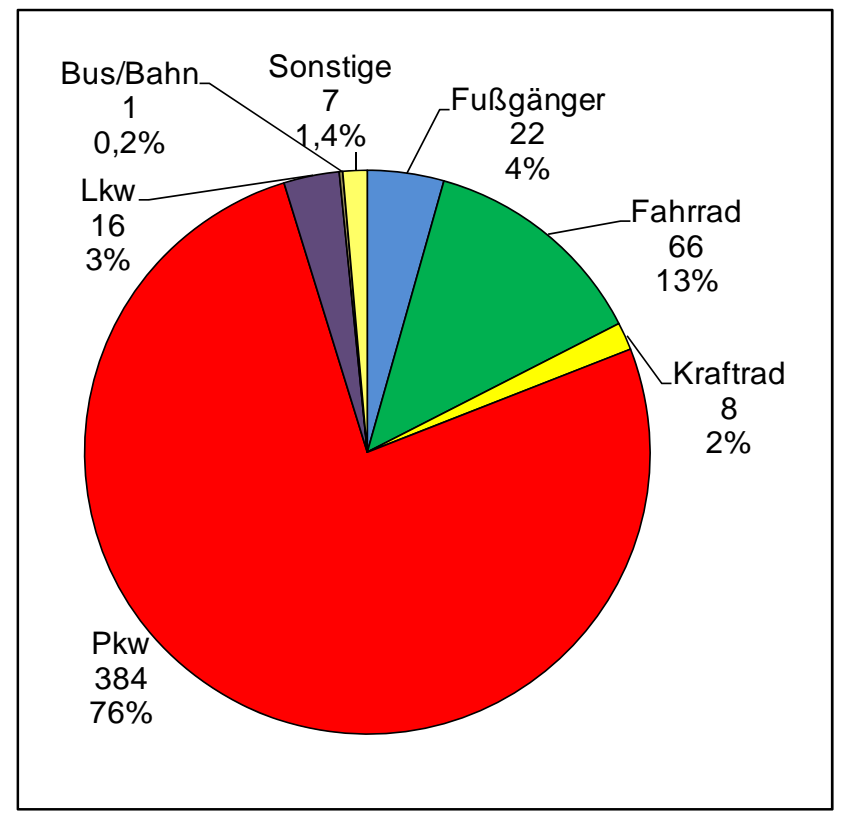

Abbildung 45: Unfallgegner bei Unfällen mit Radverkehrsbeteiligung in Fahrradstraßen in der Makroanalyse (U(P), 2008-2012; n=504; ohne Alleinunfälle Rad) ${ }^{33}$

In Fahrradstraßen sind bei Unfällen mit Radfahrerbeteiligung Pkw die mit am Abstand häufigsten Unfallgegner: Drei Viertel der Unfallgegner sind Pkw-Fahrer. Die zweithäufigsten Unfallgegner bei Unfällen mit Radverkehrsbeteiligung in Fahrradstraßen sind andere Radfahrer, allerdings liegt der Anteil hier deutlich niedriger bei rund einem Achtel. Fußgänger liegen an dritter Stelle mit 4\%, Lkw an fünfter mit $3 \%$.

${ }^{33}$ Hier sind die Alleinunfälle Rad (75 Unfälle) nicht mit dargestellt. 
Benennt man bei den Unfällen mit Radverkehrsbeteiliauna nicht nur den Unfallverursacher, sondern auch den Unfallgegner, so ergibt sich fc Beteiligter 1 | Beteiligter 2

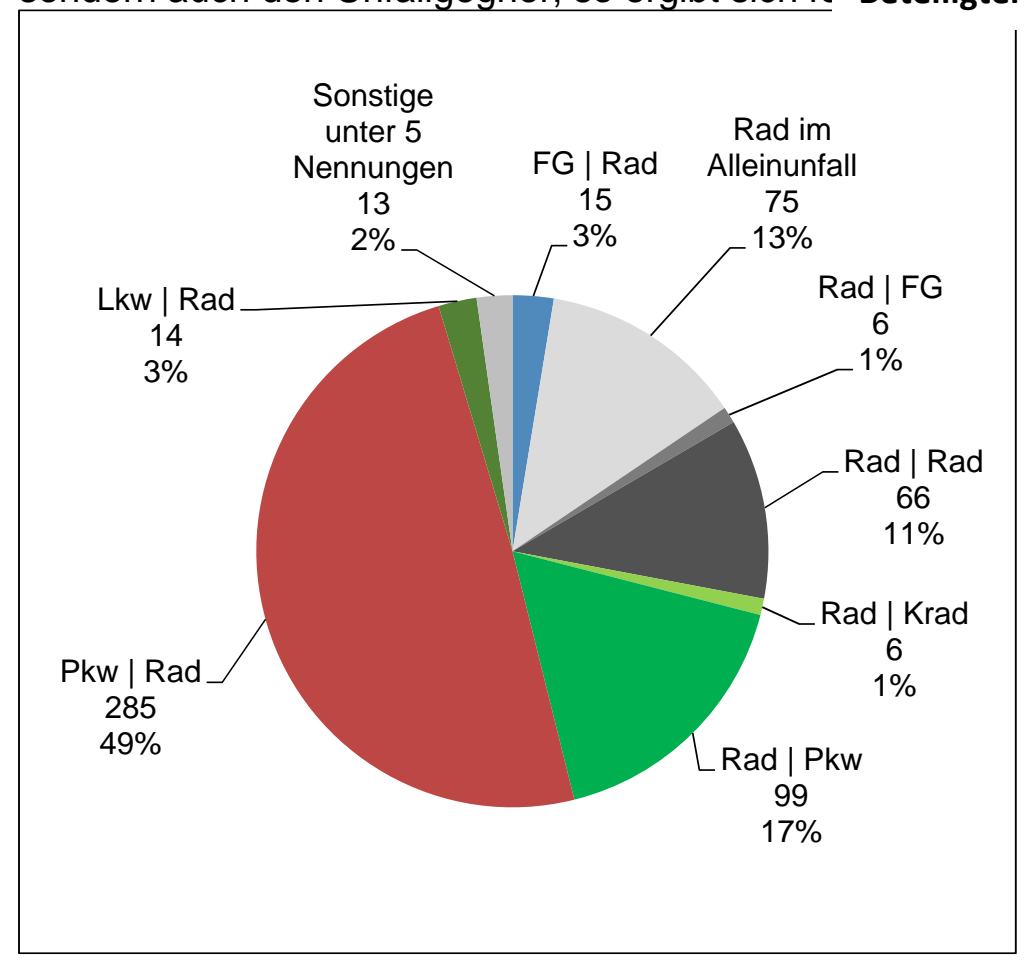

Abbildung 46 und Abbildung 47).

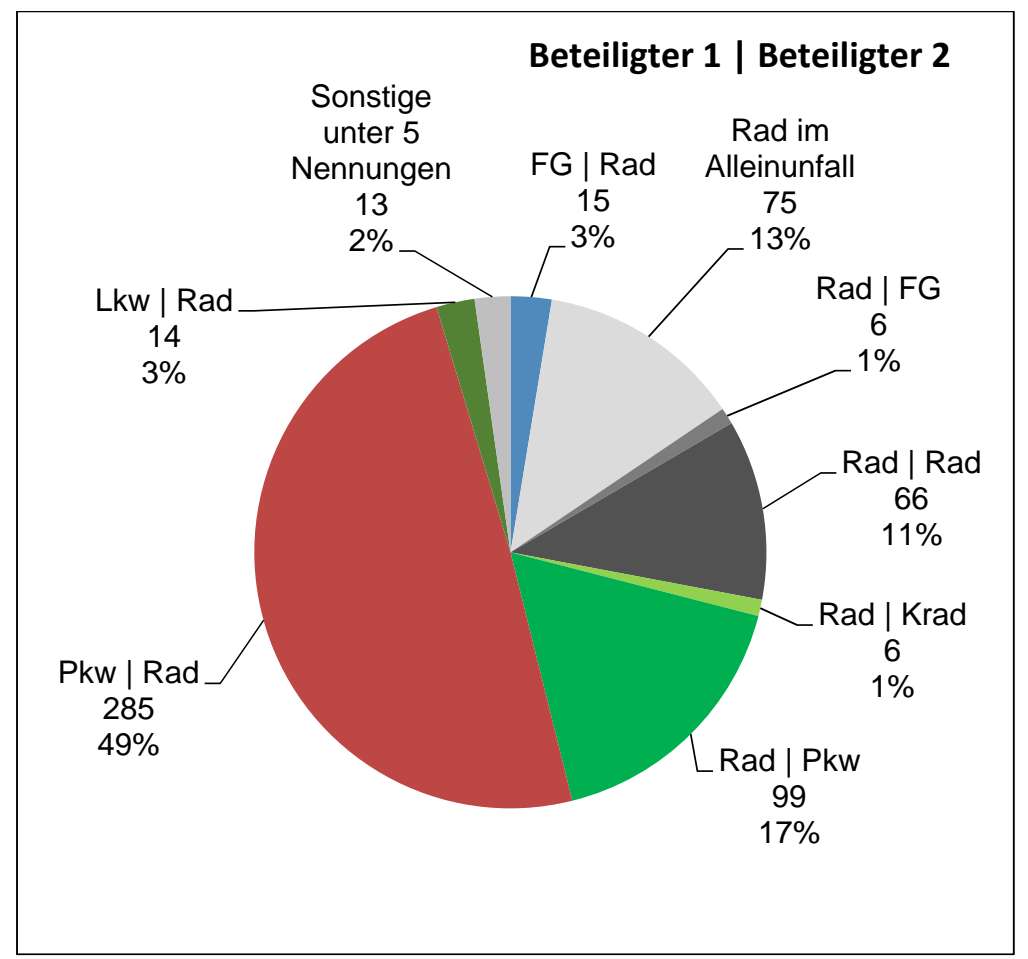

Abbildung 46: Unfallbeteiligte 1 und 2 bei Unfällen mit Radverkehrsbeteiligung in Fahrradstraßen in der Makroanalyse $(U(P), 2008-2012, n=579)$

Am häufigsten bei Unfällen mit Radverkehrsbeteiligung sind die Beteiligtenkonstellationen Pkw | Rad (49 \%) und Rad | Pkw (17 \%). Alleinunfälle Rad (13 \%) und Unfälle Rad | Rad (11 \%) sind ebenfalls häufig vertreten. Betrachtet man nur die Unfälle zwischen den Beteiligten Pkw und 
Radfahrer (in der Summe 384 Unfälle), sind zu 74 \% die Pkw-Fahrer die Hauptverursacher des Unfalls (285 Unfälle).

In der Mikroanalyse zeigt sich die häufigste Beteiligtenkonstellation Pkw | Rad noch deutlicher (59 \%). An den folgenden Stellen stehen nun die Rad | Rad-Unfälle (17\%) und die Rad | PkwUnfälle (16\%).

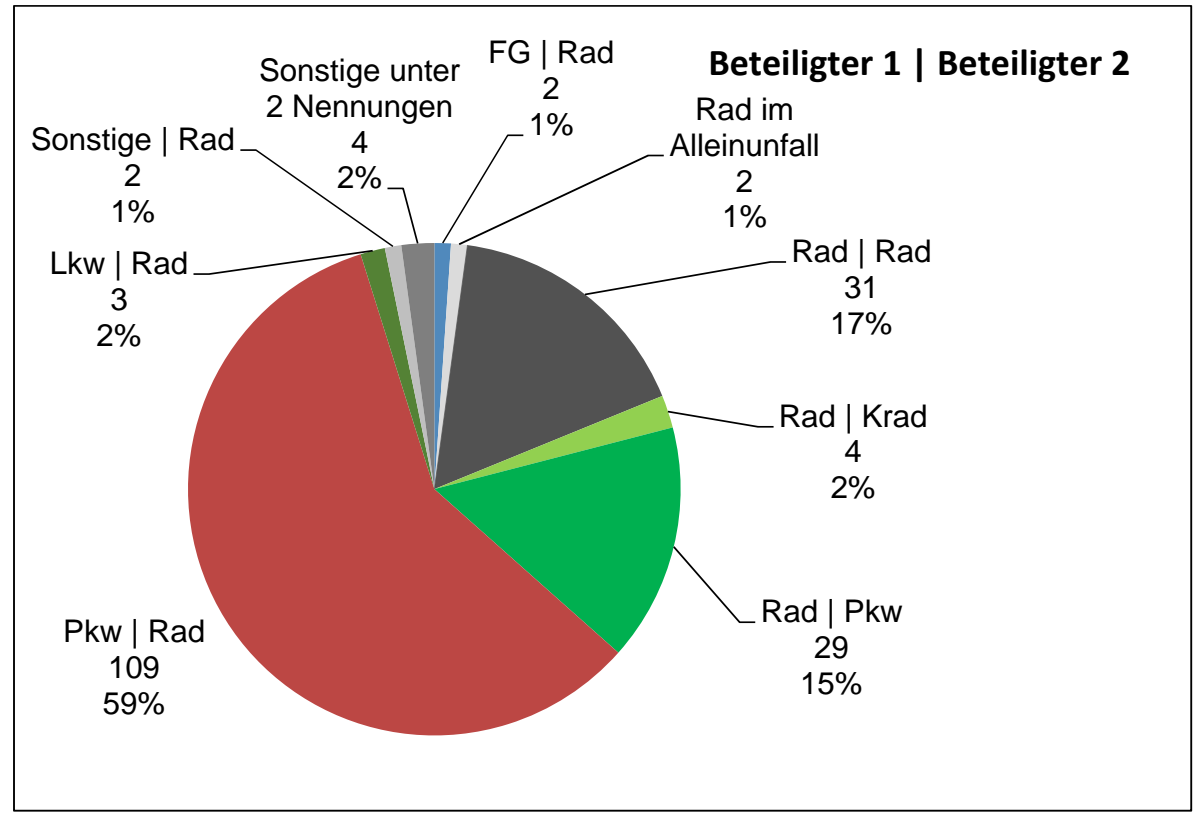

Abbildung 47: Unfallbeteiligte 1 und 2 bei relevanten Unfällen mit Radverkehrsbeteiligung in Fahrradstraßen in der Mikroanalyse $(U(P), 2008-2012, n=186)$

Sowohl auf der Strecke, als auch am Zwischenknotenpunkt sind die Beteiligtenkonstellationen Pkw | Rad dominant (siehe Abbildung 48 und Abbildung 49): 67 \% bzw. 60 \%. Am zweithäufigsten auf der Strecke und am Zwischenknotenpunkt ist die Beteiligtenkonstellation Rad | Rad (13 \% bzw. $17 \%$ ) und danach Rad | Pkw (8 \% bzw. $17 \%$ ).

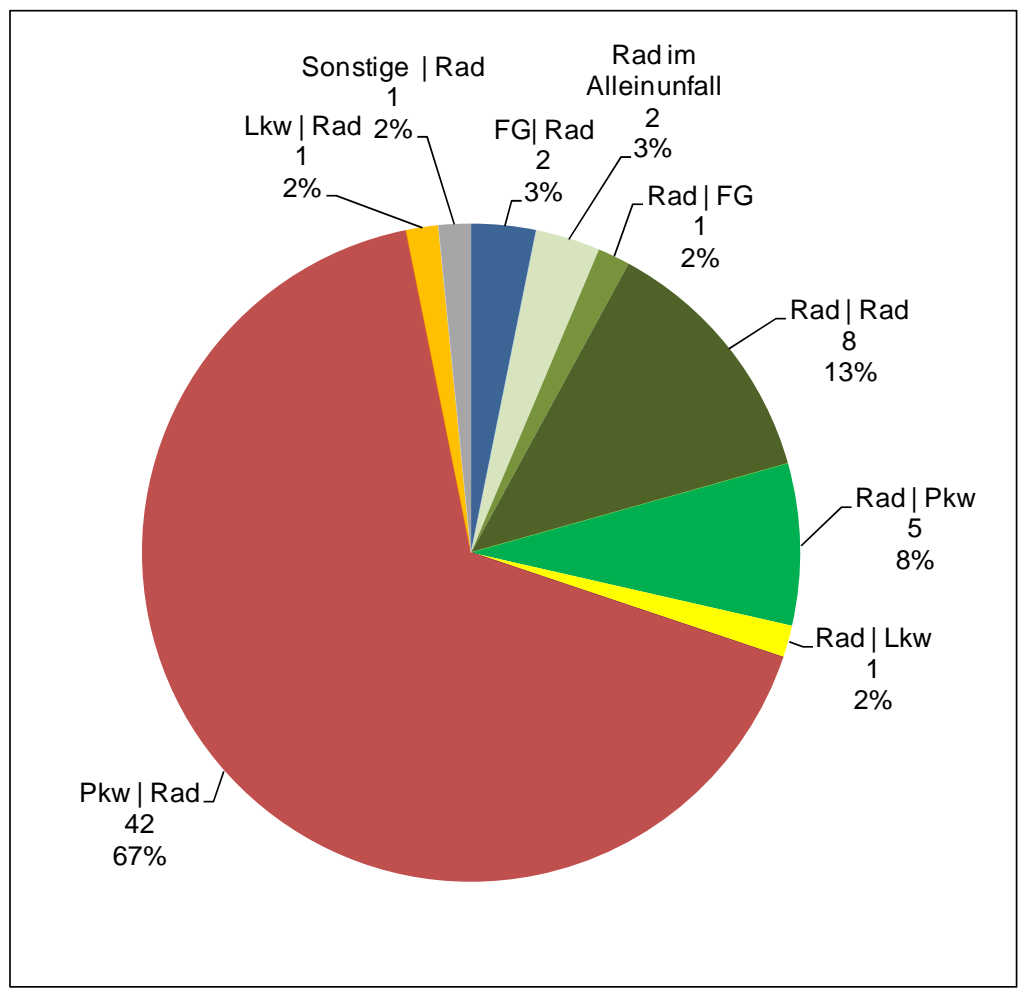


Abbildung 48: Unfallbeteiligte 1 und 2 bei Unfällen mit Radverkehrsbeteiligung in Fahrradstraßen auf der Strecke in der Mikroanalyse (U(P), 2008-2012, $n=63)$

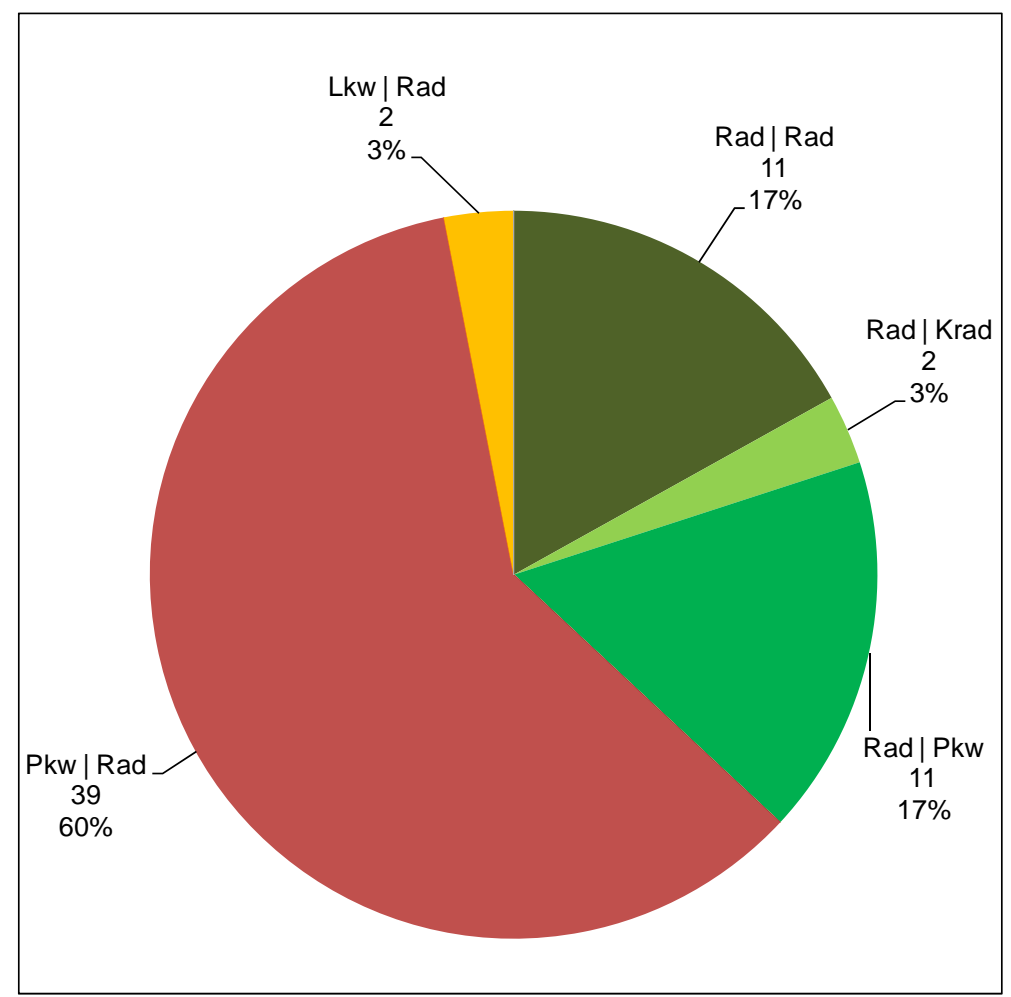

Abbildung 49: Unfallbeteiligte 1 und 2 bei Unfällen mit Radverkehrsbeteiligung in Fahrradstraßen im Zwischenknotenpunkt in der Mikroanalyse (U(P), 2008-2012, $n=65)$

Auswertungen zu den Unfallbeteiligten als Haupt-IAlleinverursacher und Mitverursacher

Die Unfallbeteiligten bei Radverkehrsunfällen in Fahrradstraßen in den Kategorien Allein- oder Hauptverursacher, Mitverursacher und Unfallbeteiligter ohne Ursache zeigt Abbildung 50 für die Makroanalyse. 


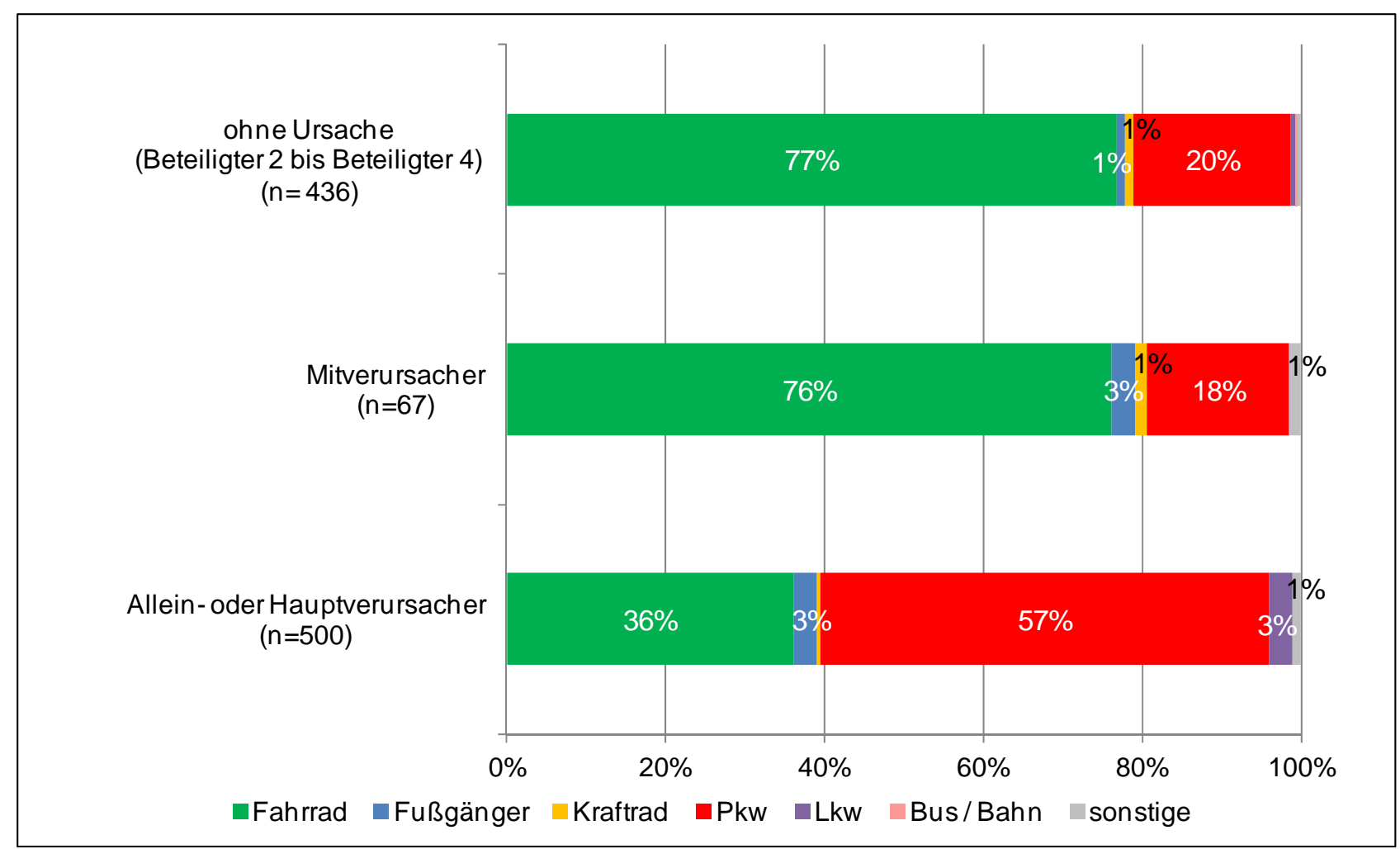

Abbildung 50: Unfallverursacher bei Unfällen mit Radverkehrsbeteiligung in Fahrradstraßen in der Makroanalyse $(U(P), 2008-2012$, ohne Alleinunfälle Fahrrad: $n=500)$

Pkw werden überwiegend als Haupt-/ oder Alleinverursacher genannt. Bei den Mitverursachern und den Unfallbeteiligten ohne Ursache überwiegen die Radfahrer zu Dreivierteln. Die Ergebnisse in der Mikroanalyse sind fast identisch: Lediglich die Krafträder sind häufiger Mitverursacher an den relevanten Radverkehrsunfällen (statt $1 \%$ dann $6 \%$ und keine Fußgänger oder sonstige Verursacher).

\subsubsection{Unfallursachen}

In der makroskopischen Unfallanalyse wurden die Unfallursachen detailliert untersucht und nach Unfallbeteiligung ausgewertet. Abbildung 51 zeigt die Unfallursachen 1 bis 3 der Unfallgegner der Radfahrer bei Unfällen mit Radverkehrsbeteiligung in Fahrradstraßen. Hierbei wurden die Radfahrer | Radfahrer-Unfälle nicht berücksichtigt. 


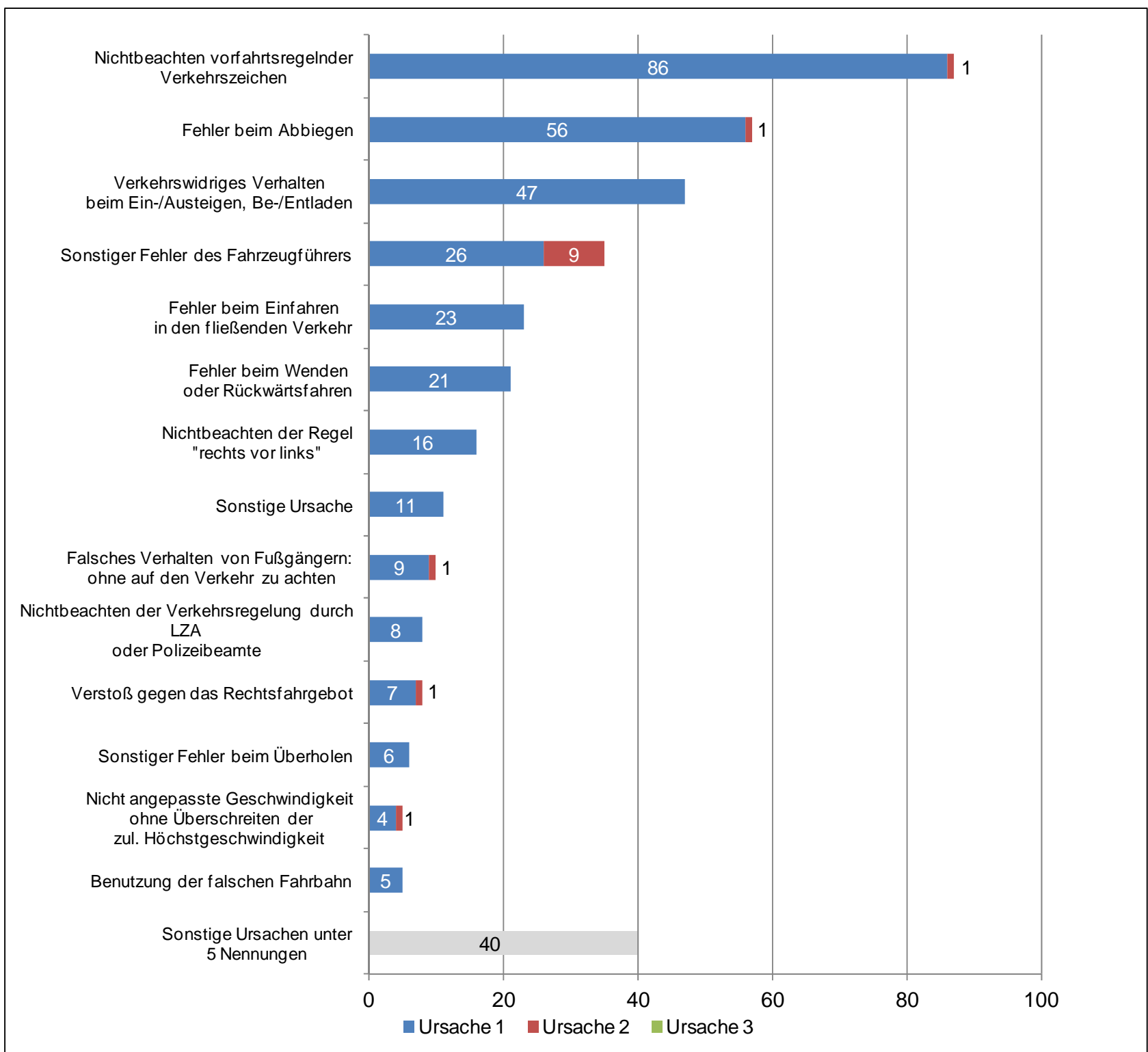

Abbildung 51: Unfallursachen der Unfallgegner von Radfahrern (alle außer Radfahrer) bei Unfällen mit Radverkehrsbeteiligung in Fahrradstraßen in der Makroanalyse (U(P), 2008-2012, n=379 Ursachen, Mehrfachnennungen möglich)

Bei Unfällen mit Radverkehrsbeteiligung in Fahrradstraßen waren Vorfahrtsverstöße die häufigsten Ursachen der Unfallgegner der Radfahrer (Vorfahrtsverstöße in der Summe ${ }^{34} \mathrm{n}=160$ bzw. $42 \%$ ). Fehler beim Parkierungsvorgang sind die zweithäufigsten Ursachen (Parkfehler in der Summe ${ }^{35}$ $\mathrm{n}=91,24 \%$ ).

Auch in der mikroskopischen Unfallanalyse waren bei den Unfallgegnern, die keine Radfahrer waren, Vorfahrtsverstöße die häufigsten Ursachen bei den relevanten Radverkehrsunfällen

34 Als Vorfahrtsverstöße wurde gewertet: Nichtbeachten vorfahrtsregelnder Verkehrszeichen, Fehler beim Abbiegen, Nichtbeachten der Regel „rechts vor links“.

35 Als Parkfehler wurden gewertet: Verkehrswidriges Verhalten beim Ein-/Aussteigen, Be-/Entladen, Fehler beim Einfahren in den fließenden Verkehr, Fehler beim Wenden/Rückwärtsfahren. 
(Vorfahrtsverstöße in der Summe ${ }^{36} \mathrm{n}=48$ ). Fehler beim Parkierungsvorgang waren die zweithäufigsten Ursachen. Abbildung 52 zeigt die Ergebnisse.

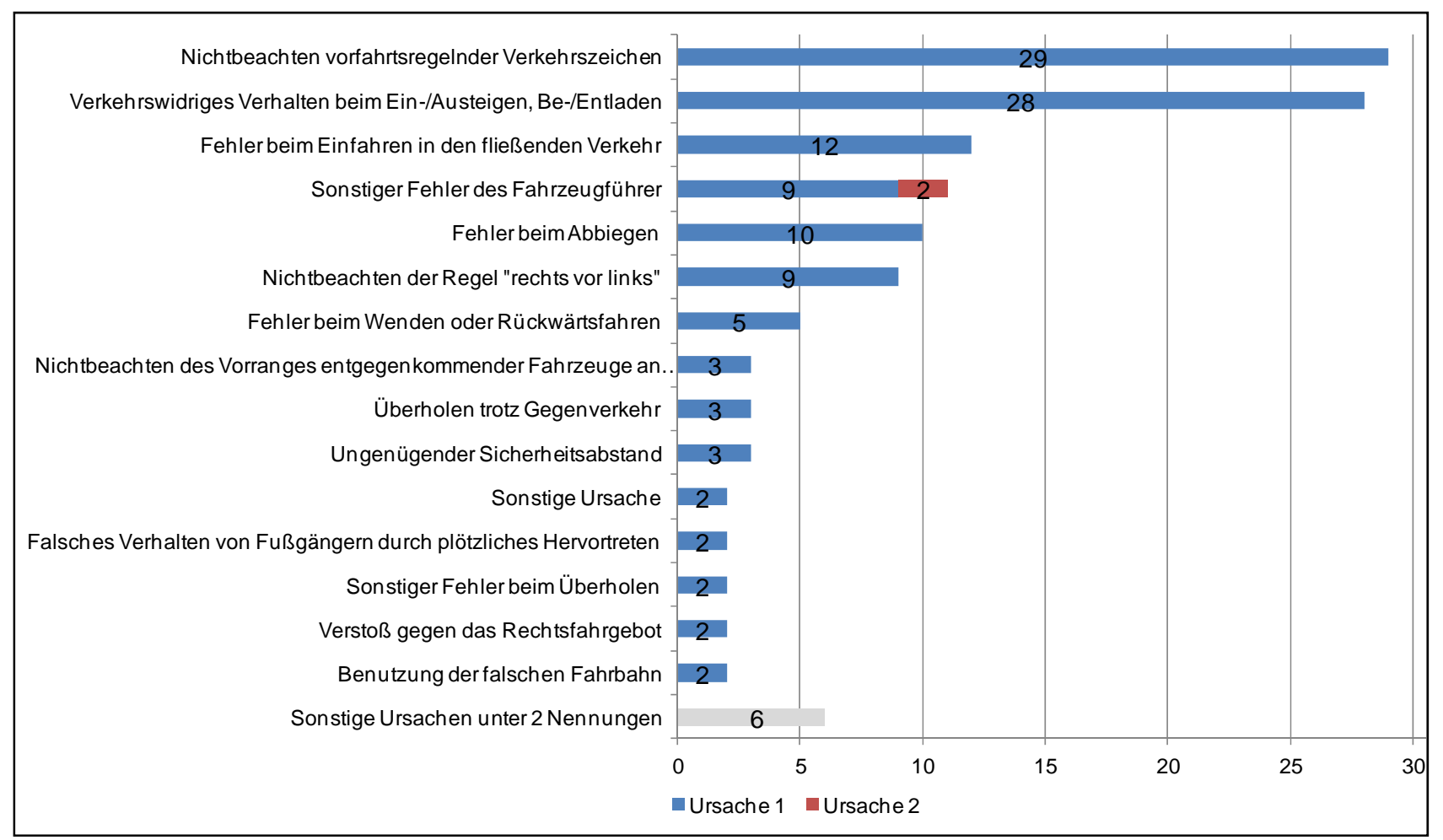

Abbildung 52: Unfallursachen der Unfallgegner von Radfahrern (alle außer Radfahrer) bei relevanten Unfällen mit Radverkehrsbeteiligung in Fahrradstraßen in der Mikroanalyse (U(P), 2008-2012, n=129 Ursachen, Mehrfachnennungen möglich)

Abbildung 53 zeigt die Unfallursachen 1 bis 3 für alle beteiligten Radfahrer an Unfällen in Fahrradstraßen.

36 Als Vorfahrtsverstöße wurde gewertet: Nichtbeachten vorfahrtsregelnder Verkehrszeichen, Fehler beim Abbiegen, Nichtbeachten der Regel „rechts vor links“. 


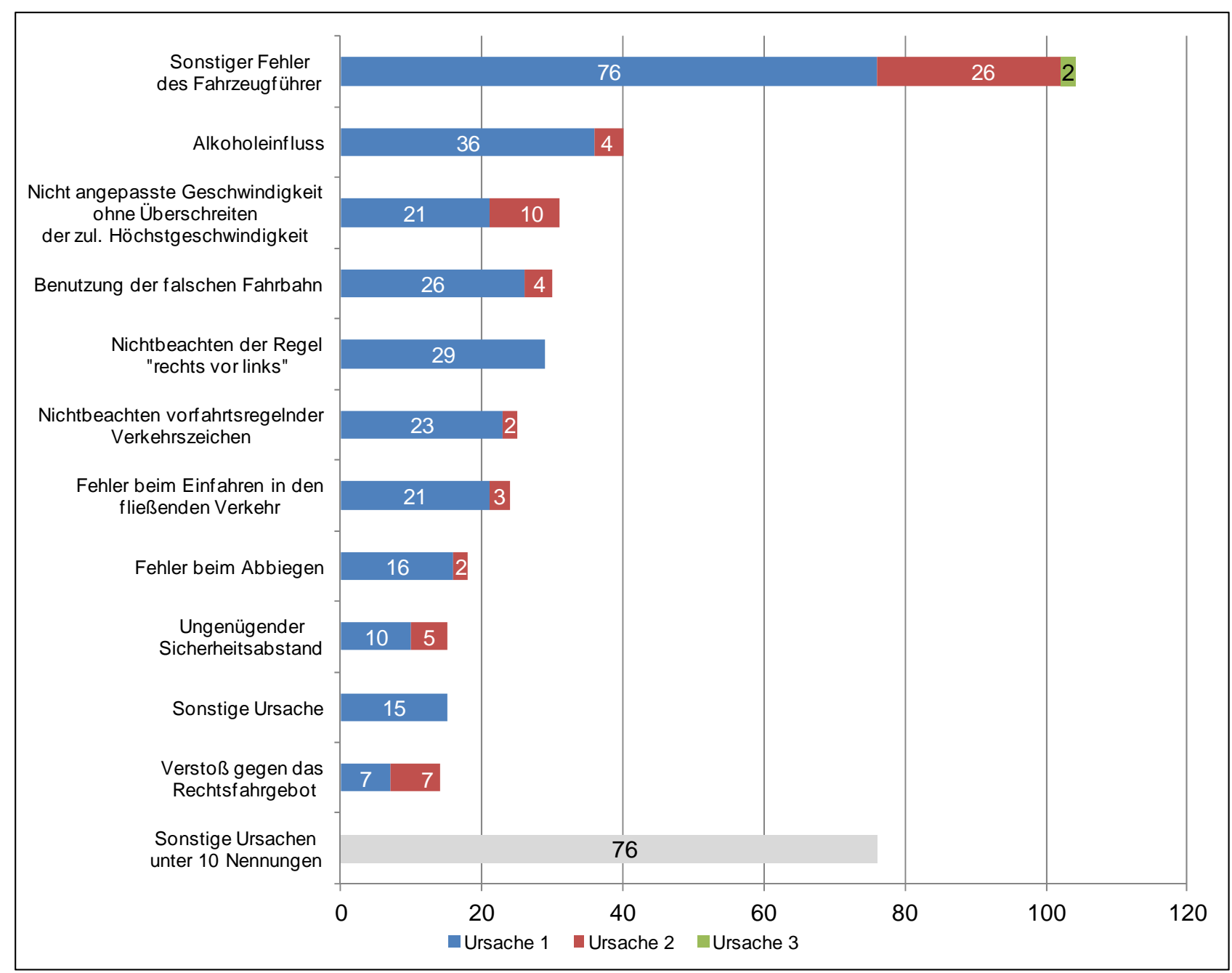

Abbildung 53: Unfallursachen aller beteiligten Radfahrer bei Unfällen in Fahrradstraßen in der Makroanalyse (U(P), 2008-2012, n=421 Ursachen, Mehrfachnennungen möglich)

Alkohol ist die häufigste „konkrete“ Unfallursache bei Radfahrern, die an Unfällen in Fahrradstraßen beteiligt sind. Eine weitere häufige Unfallursache von Radfahrern ist das Fahren mit nicht angepasster Geschwindigkeit, ohne jedoch die zulässige Höchstgeschwindigkeit zu überschreiten. Ebenso häufig wird die Unfallursache Nutzung falscher Fahrbahnteile angegeben, was Fahren auf dem Gehweg bedeuten kann; diese Ursache wurde am häufigsten angegeben, wenn Radfahrer nicht die Hauptverursacher des Unfalls waren $(n=23)$. Der Anteil der Vorfahrtsverstöße ist auch bei Radfahrern recht hoch. Sie betragen in der Summe 72 bzw. 19 \%

In der Mikroanalyse ändern sich die Unfallursachen aller beteiligten Radfahrer, da hier nur noch die relevanten Unfälle enthalten sind, d.h. Unfälle, die im Zusammenhang mit der Infrastruktur Fahrradstraße stehen. Auch bei den Unfallursachen der beteiligten Radfahrer stehen nun die Vorfahrtverstöße im Vordergrund (Vorfahrtsverstöße in der Summe ${ }^{38} \mathrm{n}=37$ ). Abbildung 54 zeigt die Ergebnisse.

37 Als Vorfahrtverstöße wurden gewertet: Nichtbeachten der Regel „rechts vor links“, Nichtbeachten vorfahrtsregelnder Verkehrszeichen, Fehler beim Abbiegen

38 Als Vorfahrtsverstöße wurde gewertet: Nichtbeachten vorfahrtsregelnder Verkehrszeichen, Nichtbeachten der Regel „rechts vor links“, Fehler beim Abbiegen. 


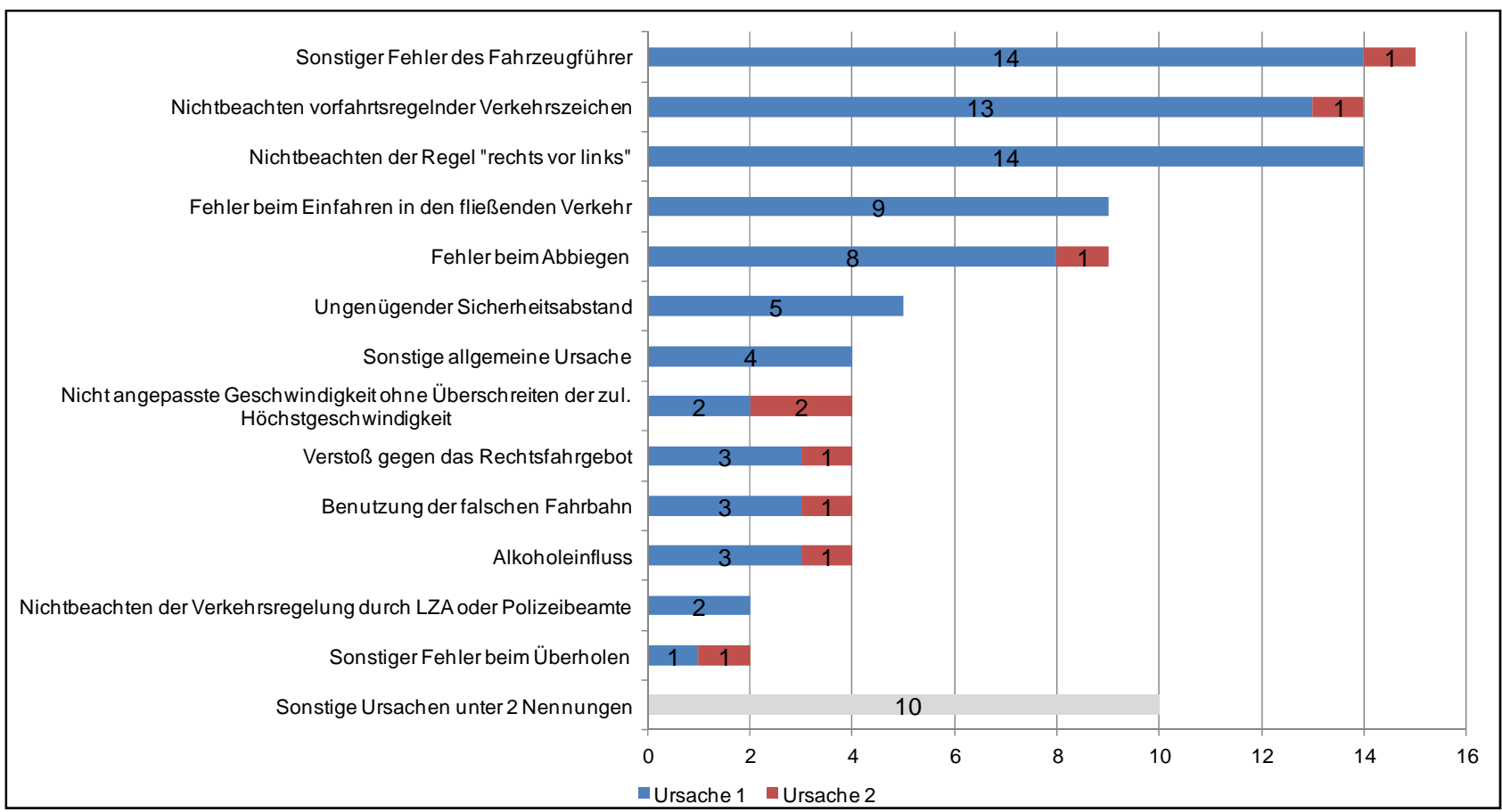

Abbildung 54: Unfallursachen aller beteiligten Radfahrer bei relevanten Unfällen in Fahrradstraßen in der Mikroanalyse (U(P), 2008-2012, n=100 Ursachen, Mehrfachnennungen möglich)

In der mikroskopischen Unfallanalyse wird bestätigt, dass die häufigsten Unfallursachen auf der Strecke Ursachen im Zusammenhang mit dem ruhenden Verkehr sind. In den Unfalltexten wird immer wieder das plötzliche Öffnen der Pkw-Tür genannt. Diese Unfälle finden häufiger in Straßen statt, in denen beidseitig geparkt wird. Dies spiegelt sich vor allem in der Anzahl der Unfälle wieder (vgl. Tabelle 4).

Tabelle 4: $\quad$ Parkregelung bei Streckenunfällen mit Radverkehrsbeteiligung in der Mikroanalyse (Unfälle mit Personenschaden (U(P), 2008-2012)

\begin{tabular}{|r|r|r|}
\cline { 2 - 3 } \multicolumn{1}{c|}{} & $\begin{array}{r}\text { Anzahl Straßen mit } \\
\text { relevanten Unfällen im } \\
\text { Zusammenhang mit dem } \\
\text { Parken }\end{array}$ & $\begin{array}{r}\text { Anzahl } \\
\text { relevanter Unfälle im } \\
\text { Zusammenhang mit dem } \\
\text { Parken }\end{array}$ \\
\hline einseitiges Parken & 13 & 15 \\
\hline beidseitiges Parken & 18 & 35 \\
\hline Gesamt & 31 & 50 \\
\hline
\end{tabular}

\subsubsection{Unfallkonstellationen}

Über die Auswertung der Unfallhergänge und Unfalltexte im Rahmen der Mikroanalyse wurden typische Unfallkonstellationen für Strecken und Zwischenknotenpunkte beschrieben.

\section{Typische Unfallkonstellationen im Zwischenknotenpunkt}

Die Vorfahrt an den Fahrradstraßen ist sehr heterogen geregelt: Es gibt Fahrradstraßen, auf denen Vorfahrt über die Verkehrszeichen VZ 301 „Vorfahrt“ und VZ 306 „Vorfahrtstraße“ angeordnet ist. Es gibt aber auch Fahrradstraßen, auf denen die Vorfahrt nicht durch Verkehrszeichen, sondern baulich durch einen abgesenkten Bordstein oder durch Markierungen angezeigt wird. Ebenso gibt 
es Fahrradstraßen auf denen die Regelung Rechts-vor-links gilt. Schließlich gibt es auch Fahrradstraßen, auf denen eine Wartepflicht gilt, die mit dem Verkehrszeichen VZ 205 „Vorfahrt gewähren" angeordnet oder durch einen abgesenkten Bordstein quer zur Fahrtrichtung baulich verdeutlicht wird.

Zu beachten ist, dass die Vorfahrtregelung innerhalb der einzelnen Fahrradstraßen an den Zwischenknotenpunkten mitunter wechselte.

Die häufigsten Unfallkonstellationen in Zwischenknoten waren:

- Missachtung des Vorrangs von Fahrradstraßen ( $n=37)$, davon

- Missachtung des VZ 301 „Vorfahrt“ und VZ 306 „Vorfahrtstraße“ ( $n=21)$

- Missachtung des abgesenkten Bordsteins $(n=12)$

- Missachtung Rechts-vor-links ( $n=4)$

- Missachtung der Wartepflicht in der Fahrradstraße $(n=14)$

Tabelle 5 führt die am häufigsten vorkommenden Unfallkonstellationen im Zwischenknotenpunkt detailliert auf, wobei die Prozentangabe in der rechten Spalte sich auf den Anteil der Unfälle bezogen auf alle untersuchten Zwischenknotenpunktsunfälle in der Mikroanalyse beziehen $(n=65)$ :

Tabelle 5: $\quad$ Häufigste Unfallkonstellationen in Zwischenknotenpunkten in der Mikroanalyse (U(P), 2008-2012, $\mathrm{n}=65$ )

\begin{tabular}{|l|l|l|l|l|l|}
\hline Fahrradstraße hat Vorrang & & (32,3\%) \\
\hline VZ 301, 306
\end{tabular}


Zu beachten ist, dass aus der Häufigkeit der Unfallkonstellationen kein direkter Rückschluss auf die sicherere oder weniger sichere Knotenpunktform gezogen werden kann da zur Ermittlung von Unfallraten die Verkehrsmengen des Kfz- und Radverkehrs nicht vorlagen. Die Auswertung der Unfallkonstellationen und ihrer Häufigkeit an den Zwischenknoten deutet dennoch auf die wichtigsten Handlungsfelder für eine sichere Knotenpunktgestaltung hin. Durch die Missachtung der Vorfahrt des Radverkehrs durch Kfz geht die größte Gefahr für den Radverkehr auf Fahrradstraßen aus. Missachtung der Vorfahrt für den Radverkehr durch den Kfz-Verkehr kommt in allen Knotenpunkttypen vor. Daraus folgt, dass in den Zwischenknotenpunkten die Vorfahrtregelung möglichst eindeutig und einheitlich verdeutlicht werden muss, um die Unfallrisiken zu minimieren.

\section{Typische Unfallkonstellationen auf der Strecke}

Die häufigsten Unfallkonstellationen auf der Strecke waren:

- Unfälle mit Längsparken $(n=24)$

- Unfälle beim Überholvorgang $(n=16)$

- Unfälle mit Schräg-/Querparken $(n=6)$.

Die Skizzen, die die typischen Unfallkonstellationen auf der Strecke verdeutlichen sollen, sind stark generalisiert: Das Parken kann sowohl einseitig, als auch beidseitig möglich sein.

Tabelle 6 führt die am häufigsten vorkommenden Unfallkonstellationen auf der Strecke detailliert auf, wobei die Prozentangabe in der rechten Spalte sich auf den Anteil der Unfälle bezogen auf alle untersuchten Streckenunfälle in der Mikroanalyse beziehen $(n=63)$ : 
Tabelle 6: $\quad$ Häufigste Unfallkonstellationen auf der Strecke in der Mikroanalyse (U(P), 2008-2012, n=63)

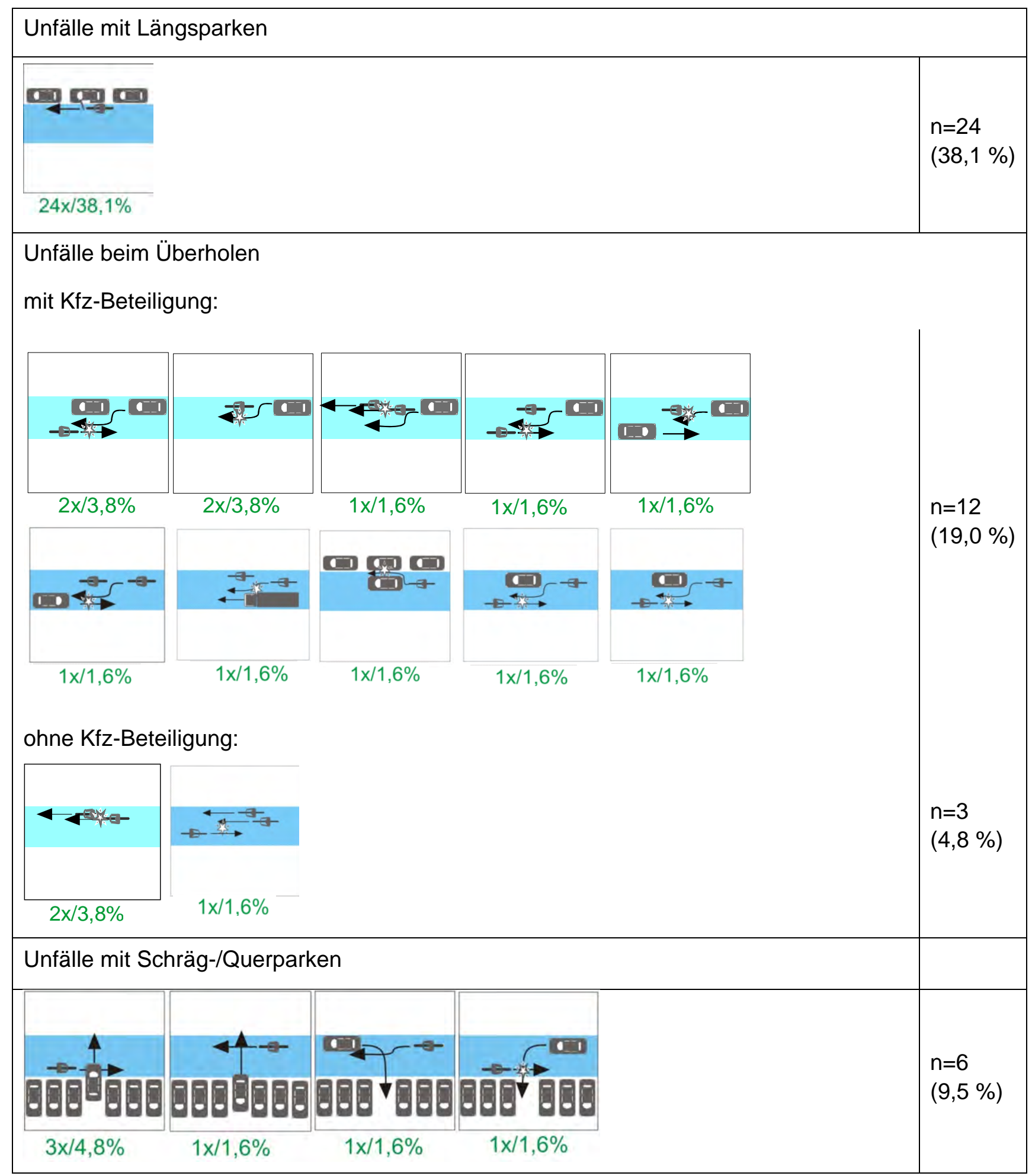

An den Streckenabschnitten, an denen in dem untersuchten Fünfjahreszeitraum mindestens ein Unfall mit Personenschaden und Radverkehrsbeteiligung stattgefunden hat, wurde die Streckencharakteristik vertieft untersucht. Auf Grund der sehr großen individuellen Unterschiede der Fahrradstraßen war eine Typisierung der Fahrradstraßen nicht zielführend. Bei der Berücksichtigung der zahlreichen Merkmale, wie Fahrbahnbreiten, Radverkehrsstärke, Kfz- 
Verkehrsstärke, Zahlenverhältnis Kfz/ Rad, Anordnung und Charakteristik ${ }^{39}$ des Parkens, KfzGeschwindigkeit sowie den unterschiedlichen baulichen Ausprägungen war es sinnvoll, von typischen Unfallkonstellationen auszugehen.

Durch die mikroskopische Auswertung wird deutlich, dass das Längsparken für Radfahrer ein Sicherheitsdefizit darstellt, insbesondere wenn nicht genügend Abstand zwischen Pkw-Tür und Fahrlinie der Radfahrer eingehalten werden kann. Diese Unfallkonstellation war die mit Abstand häufigste auf der Strecke und bedingt Konsequenzen für die Gestaltung von Fahrradstraßen.

Ein- und Ausparken bei Quer-/ und Schrägparkständen fällt als typische Unfallkonstellation nicht so stark ins Gewicht wie Unfälle bei Längsparken. Da Quer-/ und Schrägparken in den untersuchten Fahrradstraßen deutlich seltener vorkommt als Längsparken, scheinen die damit verbundenen Risiken doch auffällig zu sein. Die schlechte Sicht beim rückwärts Ausparken führt generell zu Risiken bei der Ausfahrt in den fließenden Verkehr. Dies betrifft insbesondere den Radverkehr auf der rechten Fahrbahnseite, der sehr spät vom ausfahrenden Kraftfahrer gesehen wird (siehe Tabelle 6 untere Zeile).

Typische Unfallkonstellationen auf der Strecke sind neben Unfällen mit ruhendem Verkehr, Unfälle durch Überholvorgänge, die durch Pkw vorgenommen werden. Diese Konstellationen sind allerdings sehr vielfältig. Auffällig ist allerdings, dass Überholen bei Gegenverkehr am ehesten unfallträchtig ist.

\subsubsection{Zwischenfazit zur Unfalluntersuchung in Fahrradstraßen}

In den 223 makroskopisch untersuchten Fahrradstraßen wurden im untersuchten FünfJahreszeitraum insgesamt 863 Unfälle polizeilich registriert. Dies sind im Durchschnitt knapp vier Unfälle in fünf Jahren pro Fahrstraße. Von den 223 untersuchten Fahrradstraßen waren in 42 Straßen gar keine Radverkehrsunfälle zu verzeichnen. Von den verbleibenden 181 Fahrradstraßen mit gemeldeten Fahrradunfällen, konnten 177 Fahrradstraßen in die Mikroanalyse einbezogen werden. Bei näherer (mikroskopischer) Untersuchung der Fahrradunfälle wurde zudem deutlich, dass von den 607 gemeldeten Radverkehrsunfällen nur knapp jeder Dritte einen offensichtlichen Zusammenhang mit der Infrastruktur der Fahrradstraße aufweist. Damit verblieben nur noch 75 Fahrradstraßen mit 186 zu untersuchenden Radverkehrsunfällen in der vertiefenden.

Untersuchung. Nicht relevante Unfälle fanden z.B. auf anliegenden Hauptverkehrsstraßen oder auf Privatgrundstücken statt. Auch Unfälle unter Alkoholeinfluss und Alleinunfälle wurden hier nicht gewertet (zur Definition der „relevanten Unfälle“ siehe Kapitel 2.3.1).

In Fahrradstraßen ist die Unfallschwere geringer als auf Innerortsstraßen generell. Getötete gab es in den Fahrradstraßen im Untersuchungszeitraum nicht. Dennoch liegt der Anteil schwerverletzter Verkehrsteilnehmer in den untersuchten Fahrradstraßen nur leicht unter dem bundesweiten Durchschnitt und entspricht in etwa dem Anteil der schwerverletzten Radfahrer auf Nebenstraßen.

Die Analyse von Unfalltyp, Unfallart, Unfallcharakteristik und Unfallursachen hat ergeben, dass vor allem die Missachtung der Vorfahrtsregelungen an den Knotenpunkten und die Parkregelungen zu Unfällen in Fahrradstraßen führen. Einbiegen/Kreuzen-Unfälle sind in Fahrradstraßen der dominante Unfalltyp. Dagegen sind Abbiege-Unfälle, Unfälle an Grundstücksein- und -ausfahrten sowie Unfälle mit sonstigen Ursachen deutlich unterdurchschnittlich in Fahrradstraßen vertreten, was mit der Fahrbahnführung des Radverkehrs erklärt werden kann. Unfälle durch ruhenden Verkehr sind in der Makroanalyse bereits auffällig überrepräsentiert.

39 Mit Charakteristik ist die Häufigkeit der Parkwechselvorgänge gemeint, die einen erheblichen Einfluss auf die Unfallgefährdung des parallelen Radverkehrs hat. 
In Fahrradstraßen finden deutlich mehr Unfälle mit Radverkehrsbeteiligung statt, als im bundesweiten innerörtlichen Durchschnitt. Die Verkehrszählungen an den vertieft untersuchten Straßen (siehe Abbildung 56) zeigen, dass der Radverkehr an vielen Strecken dominiert und die stärksten Fahrradstraßen bis zu 8.000 Radfahrer am Tag aufweisen. So gesehen ist es eher verwunderlich, dass der Anteil der Unfälle ohne Radverkehrsbeteiligung mit $34 \%$ (siehe Abbildung 40) noch vergleichsweise hoch ist.

Bei Unfällen mit Radverkehrsbeteiligung in Fahrradstraßen sind Pkw-Fahrer mit Abstand die häufigsten Unfallgegner. Danach rangieren die Alleinunfälle Fahrrad und die Fahrrad/FahrradUnfälle. Die Bedeutung anderer Verkehrsteilnehmer als Unfallgegner ist eher gering. Pkw sind aber nicht nur die häufigsten Unfallgegner, sondern auch die häufigsten Unfallverursacher. Auch dies ist für Fahrradstraßen ein überraschender Befund. Die Mikroanalyse hat ergeben, dass nur knapp ein Drittel der Unfälle mit Radverkehrsbeteiligung in Fahrradstraßen überhaupt einen Zusammenhang mit der Infrastruktur der Fahrradstraße hatte (zur Definition der „relevanten Unfälle" siehe Kapitel 2.3.1).

Wenn auf den zu untersuchenden Fahrradstraßen nur alle zwei Jahre ein relevanter Unfall sattfindet, hat dies Auswirkungen auf die Untersuchungsmethodik:

- Zum einen waren eine Typisierung der Fahrradstraßen nach Unfallbelastung, und ein Vergleich der Unfallraten bei der geringen Fallzahl von etwas mehr als zwei relevanten Fahrradunfällen in fünf Jahren je Fahrradstraße nicht möglich.

- Zum anderen zeigte sich dadurch natürlich, dass die Fahrradstraße ein sicheres Infrastrukturelement ist.

Vor diesem Hintergrund wurde keine Streckentypisierung sondern eine Typisierung der Unfälle durchgeführt. Die Typisierung der Unfälle, die einen Zusammenhang mit der Knoten- und Streckengestaltung der Fahrradstraße aufweisen, geben Hinweise auf die Handlungsschwerpunkte beim der Gestaltung von Fahrradstraßen. Zu diesem Zweck wurden alle 186 relevanten Fahrradunfälle nach Strecken und Knotenunfällen differenziert.

Die wenigen Unfälle mit Radverkehrsbeteiligung in Fahrradstraßen fanden zu $35 \%$ in den Zwischenknotenpunkten und zu $34 \%$ auf der Strecke statt. Die Unfälle an den Anfangs- und Endknotenpunkten hatten einen Anteil von $31 \%$, diese wurden jedoch mikroanalytisch nicht vertieft untersucht, da die Heterogenität der Gestaltung eine Einzelfallanalyse zur Folge gehabt hätte, die keine Aussagen über diesen Typ insgesamt erlaubt hätte. Der Schwerpunkt der Auswertungen lag somit auf den Zwischenknotenpunkten und den Streckenabschnitten.

Die häufigsten Unfallkonstellationen sind im Knotenpunkt die Missachtung der Vorfahrt, in der Regel durch ein Kfz. Im Zuge der Strecke geht die größte Gefährdung vom Parken aus. Hier spielt der fehlende Sicherheitsabstand eine große Rolle. Die zweite häufige Unfallkonstellation ist das Überholen im Gegenverkehr.

\subsection{Ergebnisse der Verhaltensbeobachtung}

\subsubsection{Verkehrsmengen}

Im Ergebnis bilden die untersuchten 21 Standorte ein weites Spektrum an Belastungen an den beobachteten Knotenpunkten ab (Abbildung 55). Die Kfz-Belastungen liegen in der Regel unter $3.000 \mathrm{Kfz} / 24 \mathrm{~h}$ (werktags), drei Standorte weisen höhere Werte um die $5.000 \mathrm{Kfz} / 24 \mathrm{~h}$ auf. Die Radverkehrsbelastungen weisen im Gegensatz eine wesentlich höhere Bandbreite auf. Hier reicht die Spannweite von sehr niedrigen Werten (50 Fahrräder/24h) bis zu extrem hohen Werten (rd. 4.500 Fahrräder/24h). Der absolute Höchstwert liegt an einem Kreisverkehr mit einer Belastung von 12.300 Fahrräder/24h. 

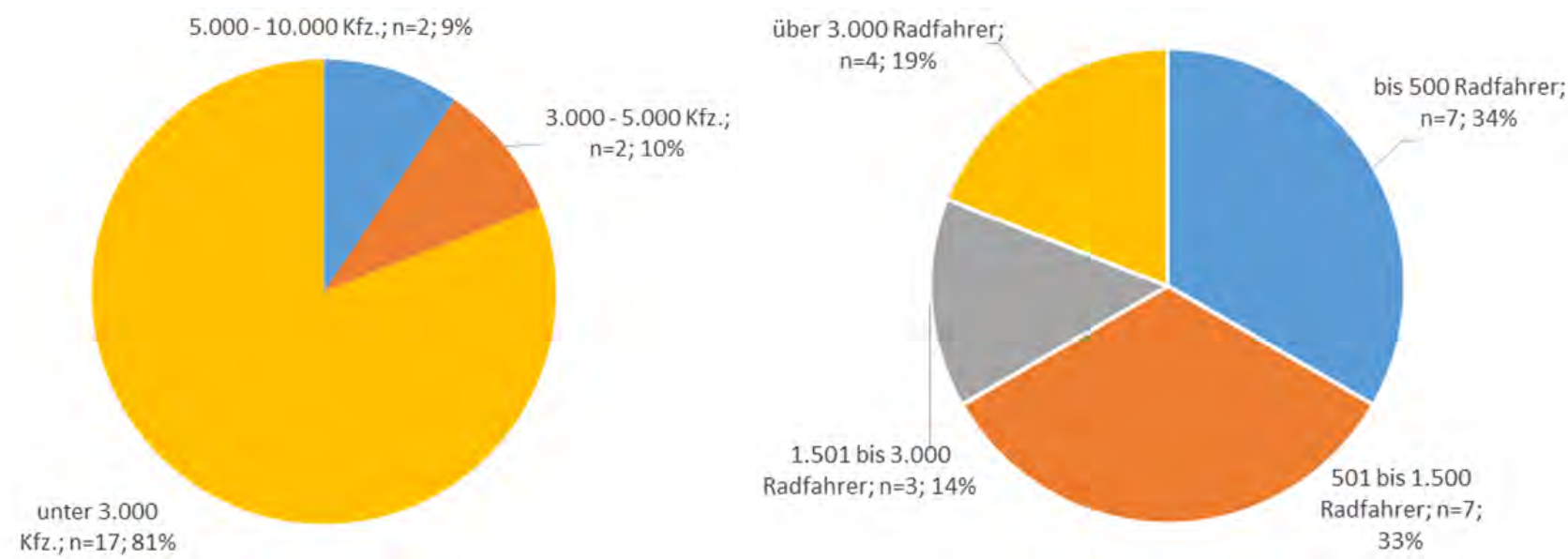

Abbildung 55: $\quad$ 24/h-Werte für Kfz- und Radverkehrsstärken in Fahrradstraßen, $\mathrm{n}=21$ Fahrradstraßen

Auch die Kombinationen von Rad- und Kfz-Verkehrsstärken variieren deutlich (Abbildung 56). So liegt der Radverkehrsanteil an 16 Standorten bei mehr als $50 \%$ der Gesamtmenge des Fahrzeugverkehrs und erreicht sogar Anteile von bis zu $96 \%$. Besonders geringe Radverkehrsanteile mit zum Teil unter $20 \%$ waren an drei Standorte zu finden.

Lediglich drei Fahrradstraßen mit geringer Kfz-Belastung waren mit „Anlieger frei“ beschildert, alle anderen Standorte mit „Kfz frei“, sodass ein Rückschluss auf den Einfluss der zugelassenen anderen Verkehrsarten hier nicht möglich war.

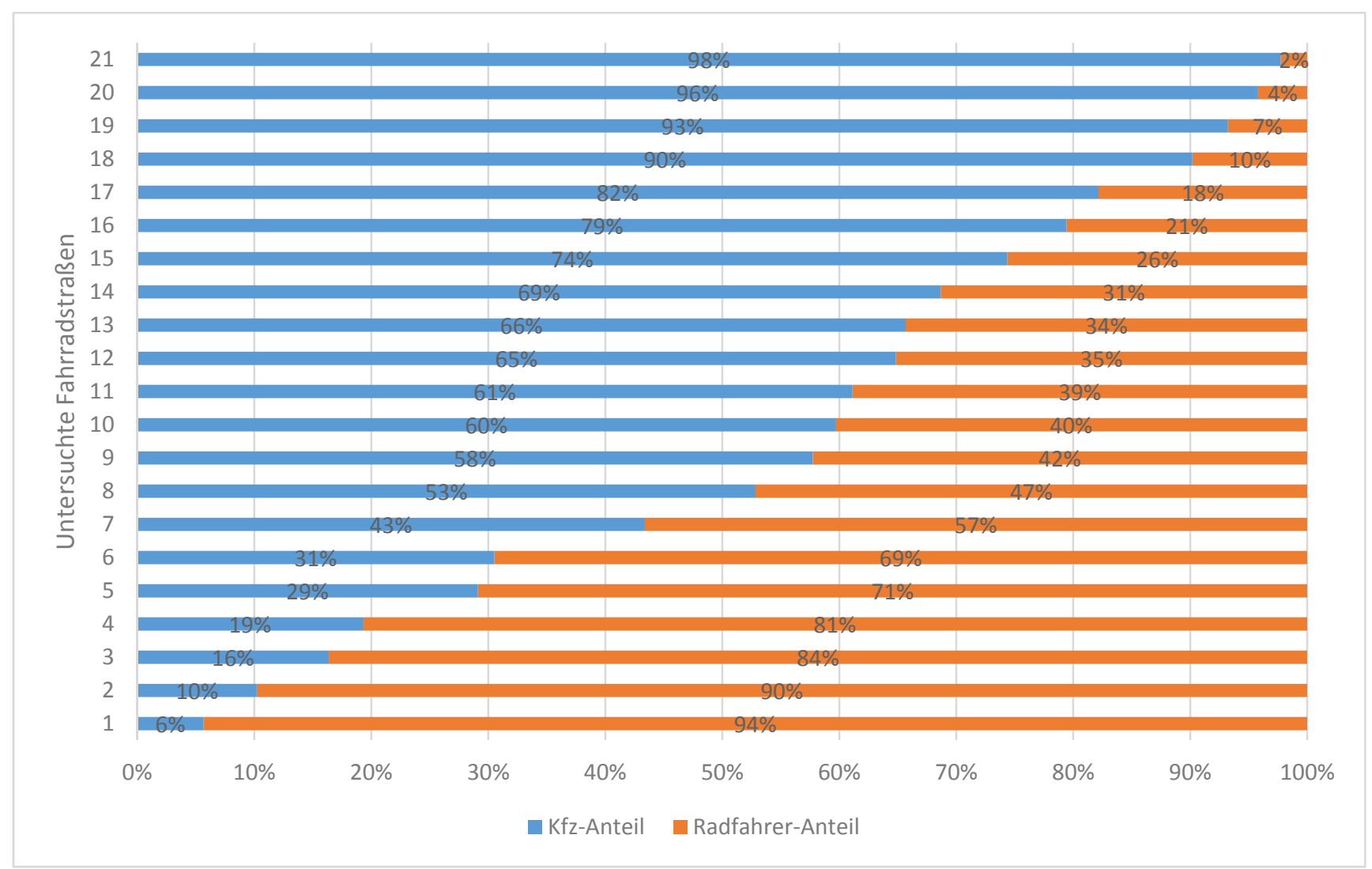

Abbildung 56: Verkehrsanteil Kfz/Radfahrer in den untersuchten Fahrradstraßen, $\mathrm{n}=21$ Fahrradstraßen

In der Auswertung der Ergebnisse lässt sich ein Zusammenhang zwischen Konflikthäufigkeit und Verkehrsstärken nicht belegen. So weisen bspw. die Straßen mit den niedrigsten 
Radverkehrsstärken von unter $500 \mathrm{Rad} / 24 \mathrm{~h}$ vergleichbar niedrige Konfliktraten auf wie die durch Radfahrer am stärksten frequentierten Fahrradstraßen (Abbildung 57).
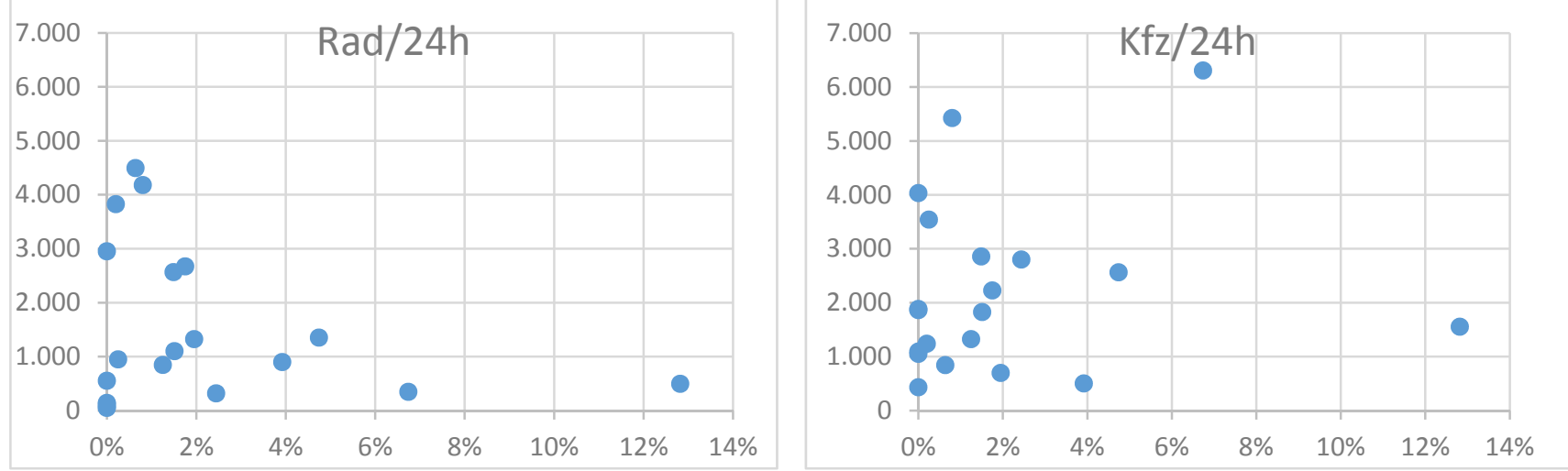

Abbildung 57: Konfliktraten und Verkehrsstärken für Rad/24h und Kfz/24h

\subsubsection{Geschwindigkeiten}

In vier Fahrradstraßen wurde neben den Verkehrsmengen zudem die Geschwindigkeit der Kfz gemessen. Von der Charakteristik her sind drei der vier Fahrradstraßen mit einer nutzbaren Fahrgasse von 3,85 m bis 5,00 m verhältnismäßig schmal. Demgegenüber steht eine wesentlich breitere Fahrradstraße mit einer nutzbaren Fahrgasse von bis zu 7,00 Metern, da hier überwiegend auf beiden Fahrbahnseiten nicht geparkt wird. Während auf den schmalen Fahrradstraßen der Radverkehrsanteil deutlich überwiegt (74 \% bis $93 \%)$ weist die breite Fahrradstraße mit $43 \%$ einen deutlich niedrigeren Radverkehrsanteil auf. Hinsichtlich der Durchfahrtserlaubnis sind alle Fahrradstraßen mit „Kfz frei“ gekennzeichnet. Im Ergebnis zeigt sich, dass $\mathrm{Kfz}$ in allen beobachteten Fahrradstraßen mitunter schneller als die maximal erlaubten $30 \mathrm{~km} / \mathrm{h}$ fahren (Abbildung 58). Die schmalen Fahrradstraßen mit einem hohen Radverkehrsanteil weisen dabei nur geringfügige Überschreitungen auf $\left(\mathrm{V}_{85}=31,2 \mathrm{~km} / \mathrm{h}\right)$ (Abbildung 59). Im Vergleich mit Geschwindigkeitsmessungen der Unfallforschung der Versicherer (UDV) in Berlin und Köln sind die in diesen Fahrradstraßen gemessenen Kfz-Geschwindigkeiten allerdings geringer als in Tempo 30-Zonen. In den drei schmalen Fahrradstraßen fuhr demnach jedes fünfte $\mathrm{Kfz}$ schneller als die erlaubten $30 \mathrm{~km} / \mathrm{h}, 6 \%$ fuhren noch schneller als $35 \mathrm{~km} / \mathrm{h}$. Im Vergleich zu diesen drei Fahrradstraßen weist die breitere Fahrradstraße mit ihrem geringeren Radverkehrsanteil eine deutlichere Geschwindigkeitsüberschreitung von $V_{85}=37,9 \mathrm{~km} / \mathrm{h}$ auf (Abbildung 60). Nicht einmal die Hälfte der gemessenen $\mathrm{Kfz}$ hielt sich an die zulässige Höchstgeschwindigkeit von $30 \mathrm{~km} / \mathrm{h}$ (60\%) und $28 \%$ fuhren schneller als $35 \mathrm{~km} / \mathrm{h}$. $8 \%$ fuhren sogar schneller als $40 \mathrm{Km} / \mathrm{h}$, mit einem Maximum von $61,2 \mathrm{~km} / \mathrm{h}$. An diesem Beispiel zeigt sich, dass in breiteren Straßen bei gleichzeitig niedrigem Radverkehrsanteil überhöhte Geschwindigkeiten auftreten können. 


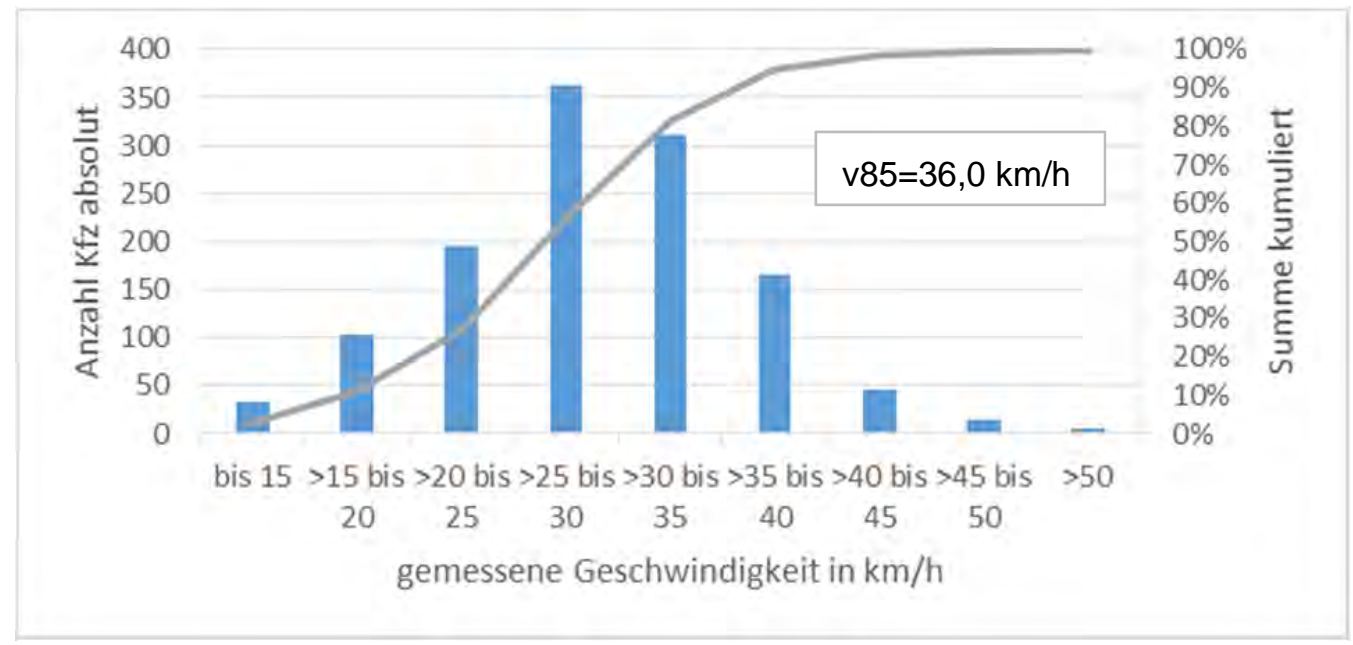

Abbildung 58: Gemessene Geschwindigkeiten auf den Streckenabschnitten in den Fahrradstraßen ( $\mathrm{n}=1.238 \mathrm{Kfz}$ in 4 Fahrradstraßen).

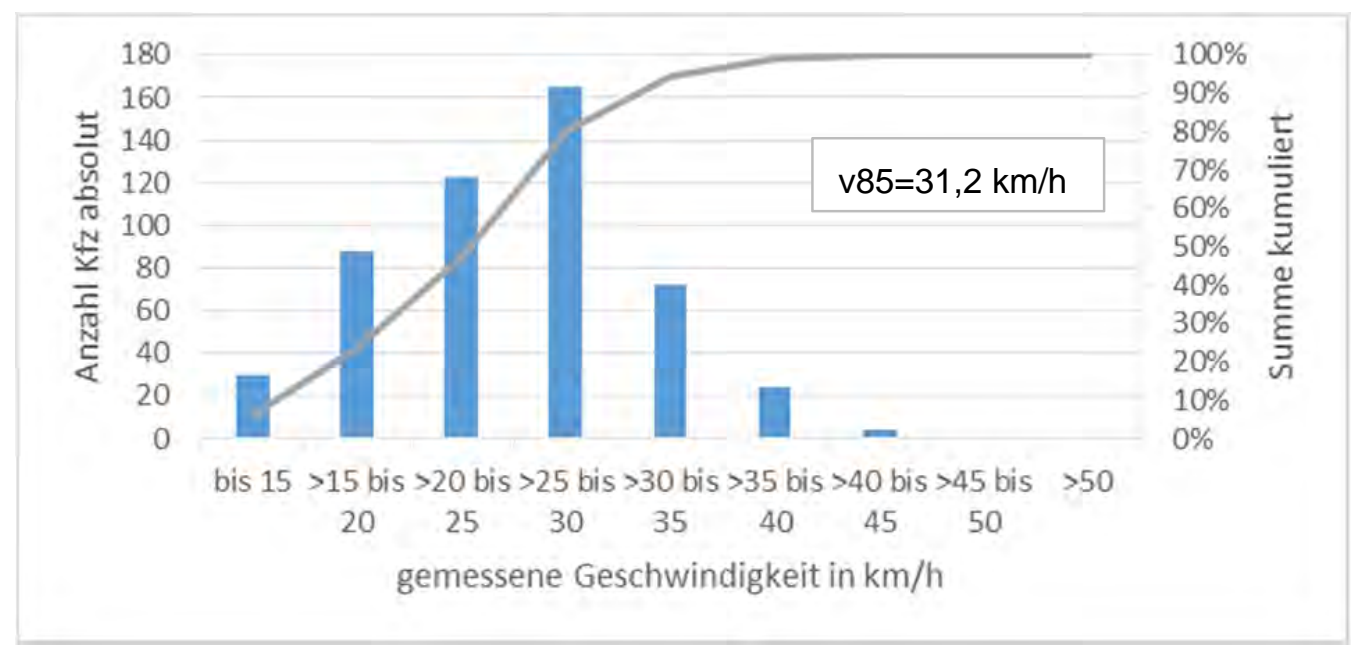

Abbildung 59: Gemessene Geschwindigkeiten in den schmalen Fahrradstraßen mit hohem Radverkehrsanteil $(n=507$ $\mathrm{Kfz}$ in 3 Fahrradstraßen).

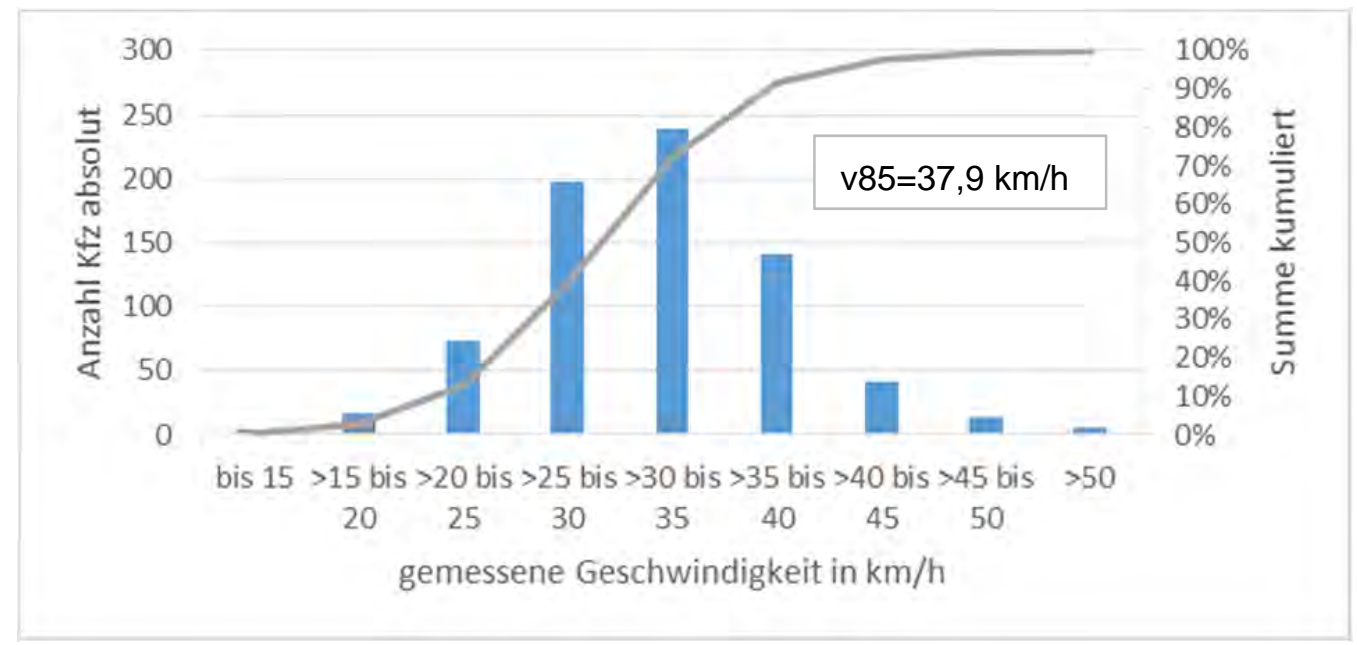

Abbildung 60: Gemessene Geschwindigkeiten in der breiten Fahrradstraße mit geringerem Radverkehrsanteil $(n=737$ $\mathrm{Kfz}$ in einer Fahrradstraße). 


\subsubsection{Interaktionen}

Nach den im Methodikteil erläuterten Definitionen von Interaktionen zwischen $\mathrm{Kfz}$ und Rad sowie leichten und schweren Konflikten wurden in den Videoaufnahmen auf den 16 Fahrradstraßen, bei denen die Knotenpunkte beobachtet wurden, in Summe 5.475 Interaktionen ermittelt ${ }^{40}$. Davon wurden 71 Interaktionen als Konflikte bewertet, darunter drei als schwere Konflikte, die jedoch ohne weitere Folgen blieben. Dies entspricht einer durchschnittlichen Konflikthäufigkeit von 1,3\% bei einem Maximum von 12,8 \%. Auf zwei Fahrradstraßen lag die Konflikthäufigkeit über 5 \%, neun Fahrradstraßen wiesen einen besonders geringen Konfliktanteil von unter $1 \%$ auf, davon wurden auf sechs Straßen keine Konflikte beobachtet.

Auf den untersuchten Streckenabschnitten war die Konflikthäufigkeit mit durchschnittlich 0,8 \% nochmals geringer als in den Knotenpunkten. Bei 1.079 beobachteten Interaktionen wurden lediglich 9 leichte Konflikte registriert.

Interaktionen verlaufen demnach in der weitaus größeren Anzahl aller Fälle ohne Konflikte ab und schwere Konflikte bilden die Ausnahme. Kommt es zu Konfliktfällen, so zeigt sich, dass sich die Verkehrsteilnehmer in der Regel arrangieren bzw. es ist zu beobachten, dass sowohl Radfahrer als auch Kfz-Fahrer ein generelles Sicherungsverhalten aufweisen, das es innen ermöglicht, im Falle eines drohenden Konfliktes rechtzeitig zu reagieren und so schwerere Konflikte vermieden werden können. Der Großteil der Konflikte an Knotenpunkten (61 von 71 Konflikten, 86 \%) stand im Zusammenhang mit der Vorfahrtsregelung. $10 \%$ der Konflikte waren auf eine falsche Benutzung der Fahrbahn ${ }^{41}$ zurückzuführen. Eine weitere Konfliktquelle stellen Fußgänger die unachtsam die Straße überqueren dar. Diesbezüglich ließen sich drei Konflikte beobachten.

Auf den Streckenabschnitten fallen von den neun beobachteten Konflikten sechs in die Kategorie „Konflikte im Längsverkehr“. Zwei Konfliktsituationen entstanden durch ruhenden Verkehr im Zusammenhang mit sich öffnenden Türen von haltenden Kfz, der Radfahrer musste jeweils ausweichen. Ein weiterer Konflikt entstand, weil ein Lkw die Fahrbahn versperrte und die Radfahrer über den Gehweg auswichen (Abbildung 61).

Beim Vergleich der sich tatsächlich an den Standorten ereigneten Unfälle mit der Anzahl der Konflikte konnten keine Zusammenhänge zwischen den Unfall und Konfliktzahlen festgestellt werden.

\footnotetext{
${ }^{40}$ Der Fahrrad-Kreisverkehr wurde als Sonderfall nicht berücksichtigt, da es hier hauptsächlich zu Interaktionen zwischen Radfahrern kommt.

${ }^{41}$ Z.B. Mitbenutzung der Gegenfahrbahn durch Kfz beim Rechtsabbiegen, Wenden von Kfz im Knotenpunkt.
} 


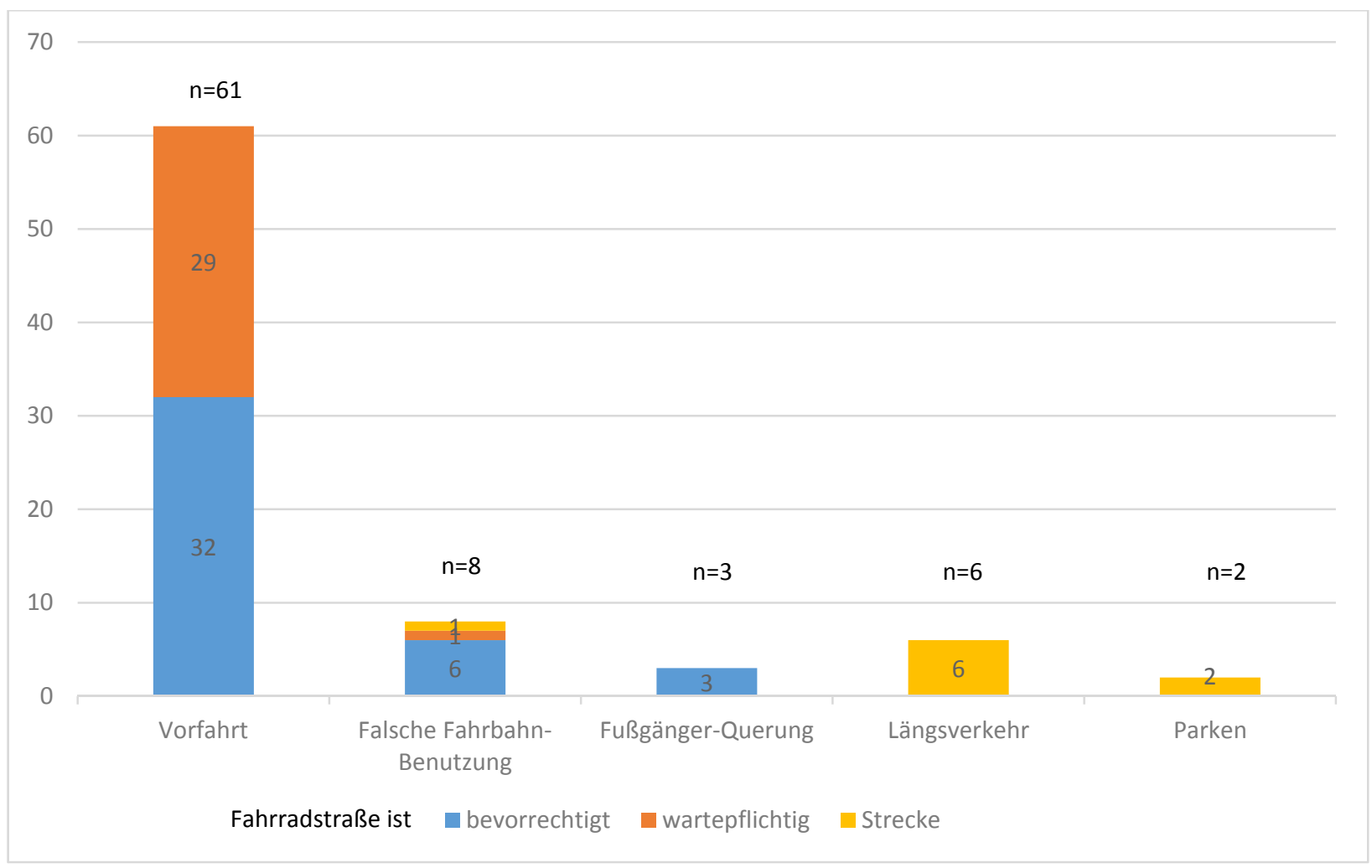

Abbildung 61: Konflikte nach Fehlerarten (Strecke und Knotenpunkte), $n=80$ Konflikte

\section{Vorfahrtregelung und Konflikthäufigkeit}

Betrachtet man nur die Vorfahrtsregelung, so fällt auf, dass auf den bevorrechtigten Fahrradstraßen etwas häufiger Konflikte entstehen (58 \%) als wenn die Fahrradstraße wartepflichtig (42 \%) ist (vgl. Abbildung 61). Verursacher waren mehrheitlich die jeweils wartepflichtigen Parteien, in den bevorrechtigten Fahrradstraßen die Kfz (zu $91 \%$ ) und in den wartepflichtigen ${ }^{42}$ Fahrradstraßen die Radfahrer zu $97 \%$.

Die detaillierte Betrachtung der untersuchten Knotenpunkte von Fahrradstraßen ergaben folgende Ergebnisse:

- An den Knotenpunkten mit Rechts-vor-links-Regelung (4 Knotenpunkte) wurden keine Konflikte beobachtet.

- Im Sonderfall von zwei sich kreuzenden Fahrradstraßen, bei der eine Fahrradstraße baulich bevorrechtigt ist, gab es keinen Unterschied in der Konflikthäufigkeit zwischen $\mathrm{Kfz}$ und Fahrrad (2 Knotenpunkte).

- An den Knotenpunkten der bevorrechtigten Fahrradstraßen (6 Knotenpunkte) waren Kfz Hauptverursacher der Konflikte, obwohl die untergeordneten Zufahrten eindeutig mit Wartelinie und Zeichen 205 StVO geregelt sind. Zum Teil sind diese Knotenpunkte zusätzlich noch aufgepflastert, farblich in Rot markiert oder mit dem Fahrradsymbol als Markierung aufgebracht. Die Missachtung der Vorfahrt durch Kfz ließ sich demnach nicht auf die Gestaltung der Knotenpunkte zurückführen.

- Auf einer sehr langen Fahrradstraße mit wechselnder Vorfahrt wurden alle beobachteten Konflikte durch Radfahrer an wartepflichtigen Knotenpunkten verursacht. Die Gestaltung

\footnotetext{
${ }^{42}$ umfasst Z 205 und Rechts-vor-links.
} 
der untergeordneten Zufahrten war auch hier in allen Fällen eindeutig und klar verständlich (Wartelinie, gute Sichtverhältnisse).

Aufgrund der sehr geringen Anzahl der beobachteten Konflikte lassen sich ausschließlich aus der Verhaltensbeobachtung keine allgemeinen Empfehlungen für die Vorfahrtregelung an Knotenpunkten von Fahrradstraßen ableiten.

Auch ein Zusammenhang zwischen Konflikthäufigkeit und infrastrukturellen Merkmalen wie Markierung und baulicher Gestaltung der Fahrradstraße konnte genauso wenig festgestellt werden, wie ein Zusammenhang mit der Verkehrsbelastung, Interaktionshäufigkeit oder Konflikthäufigkeit. Als Ergebnis der Videobeobachtung ist vielmehr davon auszugehen, dass die Konflikthäufigkeit sehr stark vom individuellen Verhalten der Verkehrsteilnehmer abhängt.

\section{Beispiele von Konflikten:}

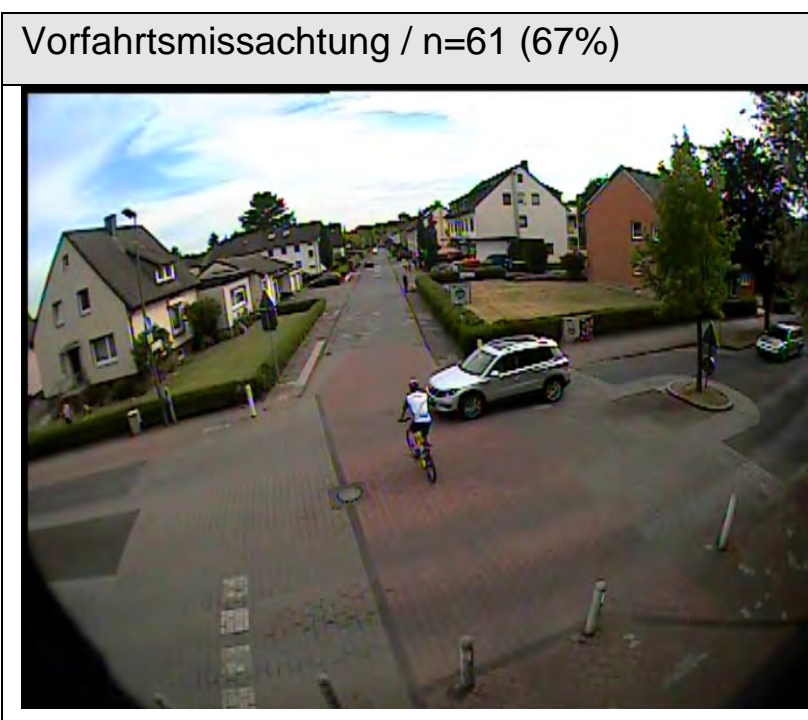

Abbildung 62: Wartepflichtiges Kfz kommt durch verspätetes Bremsen im Fahrbereich des Radfahrers zum Stehen, Radfahrer weicht aus.

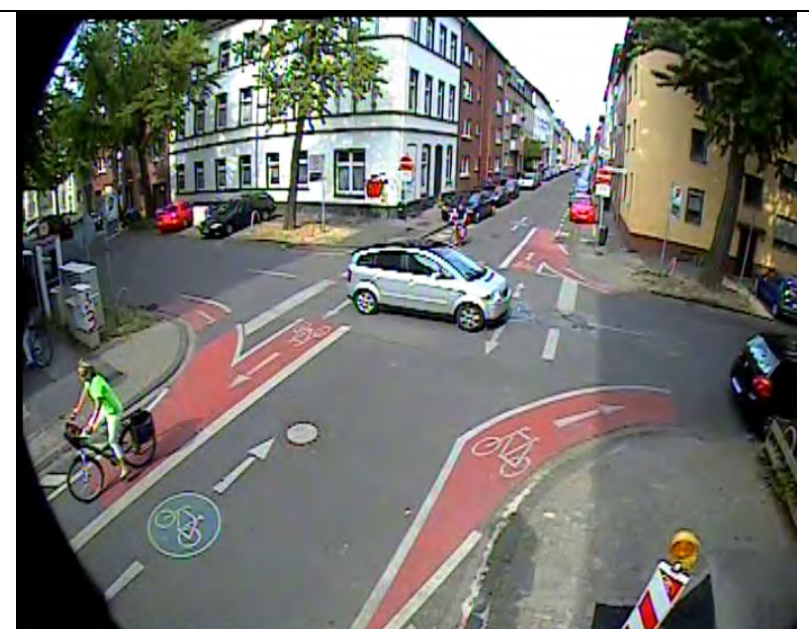

Abbildung 64: Wartepflichtiger Pkw quert Fahrradstraße und nimmt Radfahrer die Vorfahrt, dieser reduziert seine Geschwindigkeit.

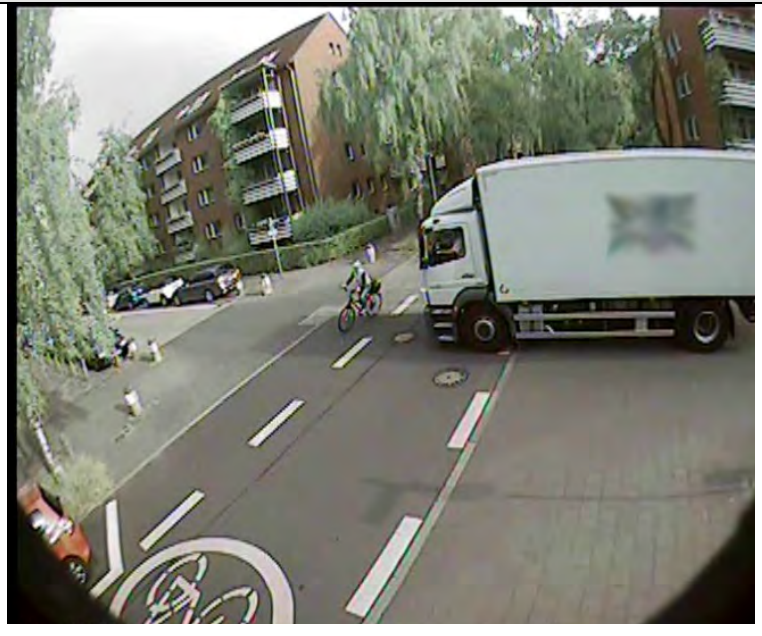

Abbildung 63: Wartepflichtiger Lkw fährt in Kreuzung ein, obwohl Radfahrer sich nähert, abrupte Bremsung des Lkw verhindert Kollision.

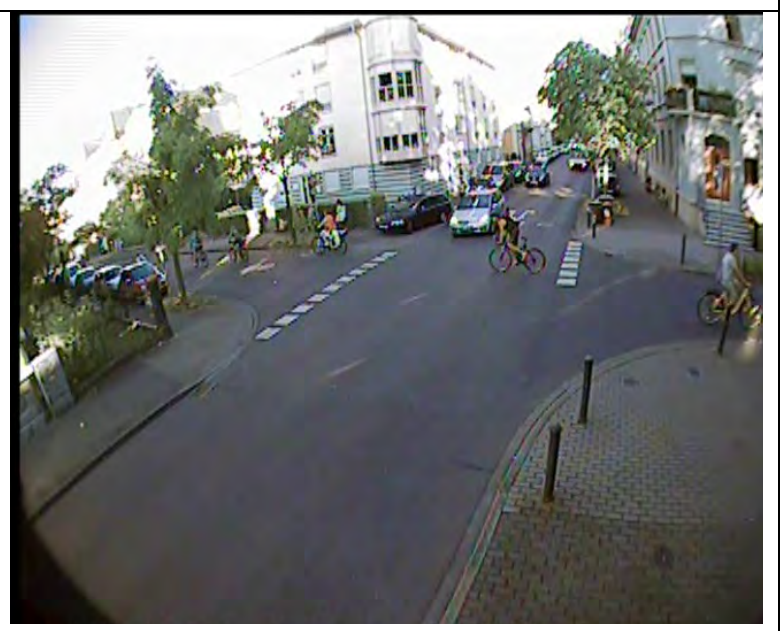

Abbildung 65: Wartepflichtiger Radfahrer auf der Fahrradstraße nimmt Pkw die Vorfahrt, dieser bremst leicht. 

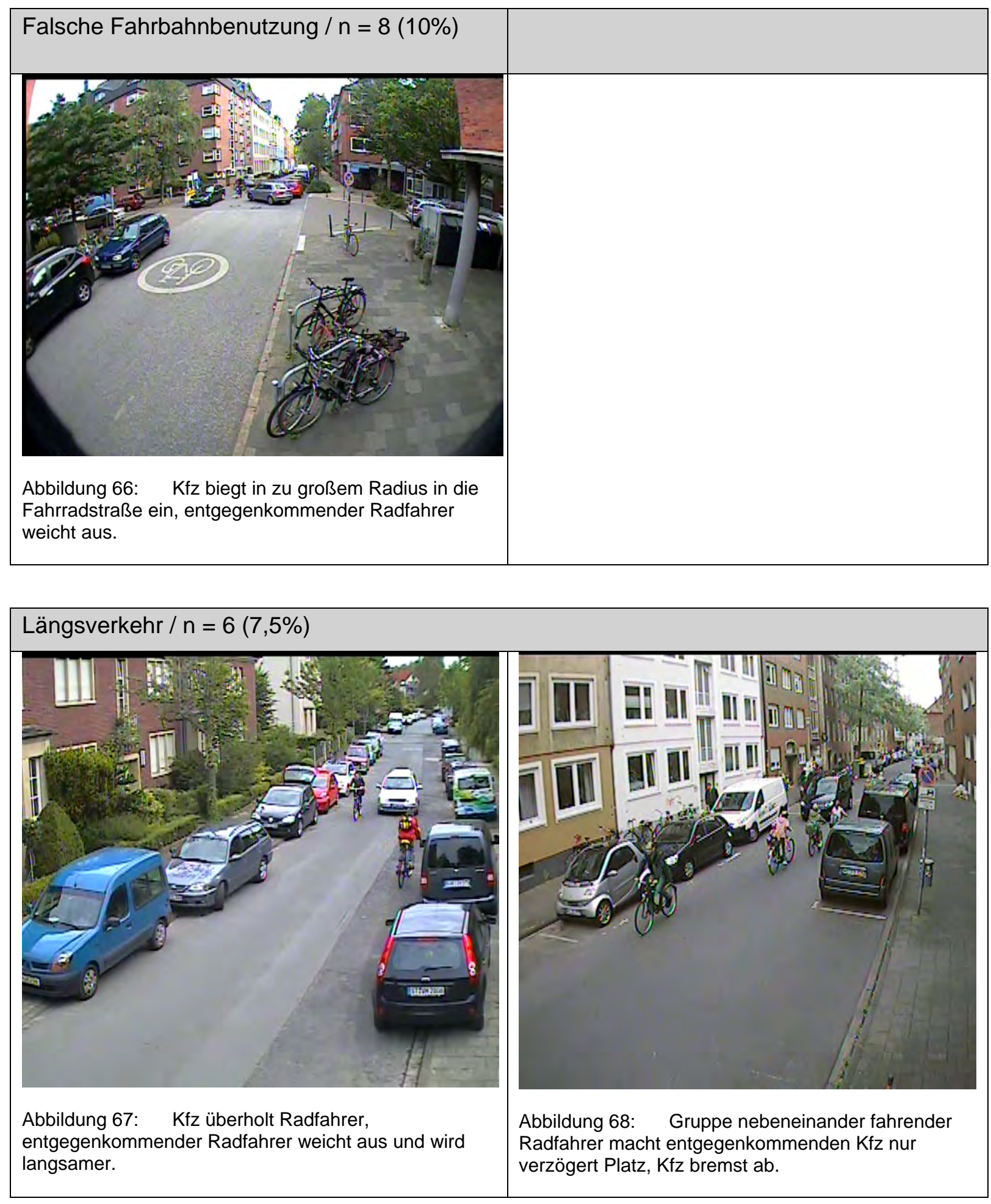

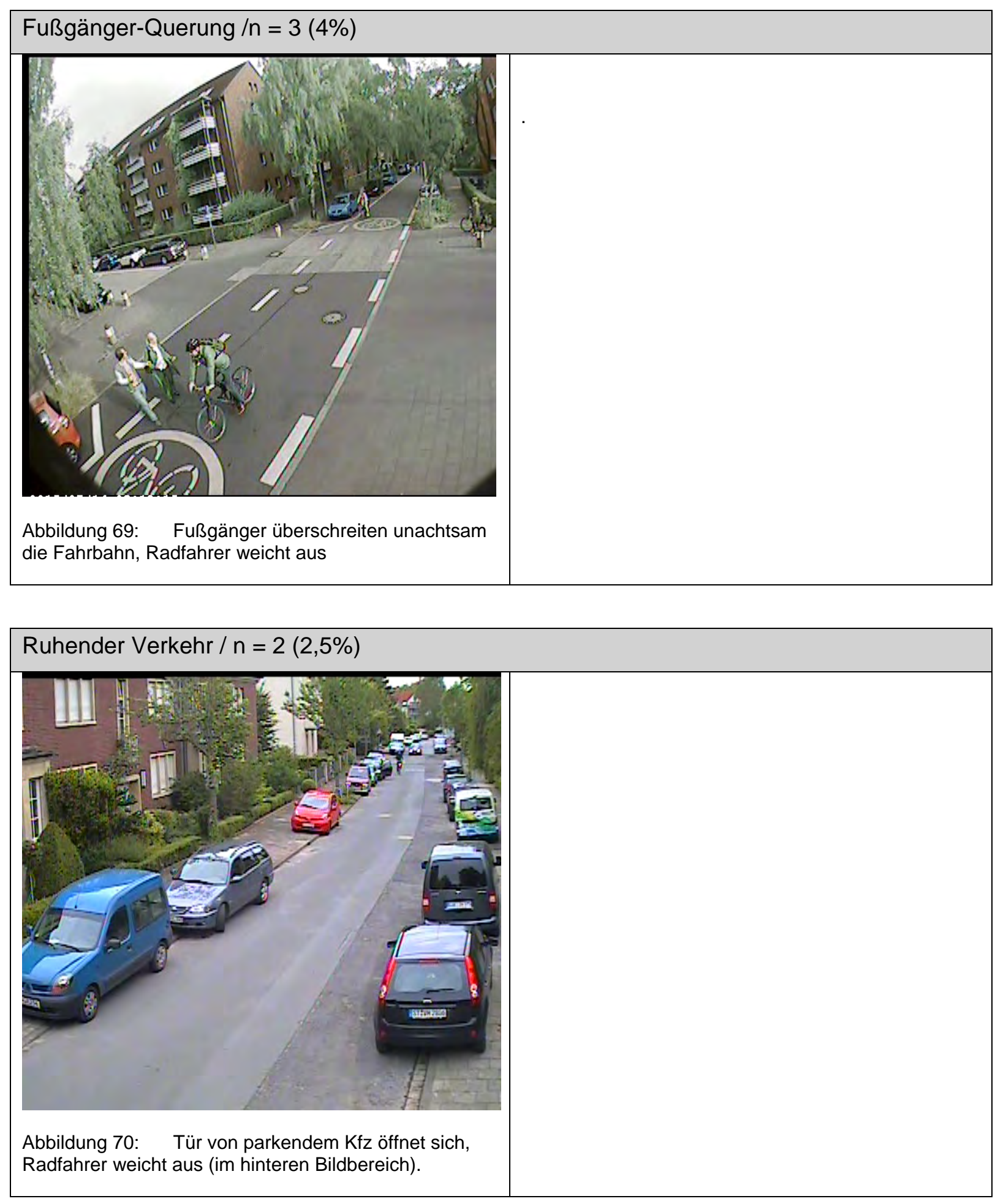

\subsubsection{Zwischenfazit der Verhaltensbeobachtung}

Die Auswertung der Verhaltensbeobachtung hat ergeben, dass in Fahrradstraßen die Kfz geringfügig schneller als die zulässige Höchstgeschwindigkeit von $30 \mathrm{~km} / \mathrm{h}$ fahren. Die gemessenen Geschwindigkeitsüberschreitungen lagen zwischen $10 \%$ und $25 \%$. Des Weiteren zeigte sich, dass der überwiegende Großteil der Interaktionen (99 \%) konfliktfrei verläuft. Treten Konflikte auf, dann in Knotenpunkten im Zusammenhang mit der Vorfahrtsregelung. Ein Zusammenhang zwischen infrastrukturellen Merkmalen und der Anzahl der Konflikte konnte dabei nicht nachgewiesen werden. Die typischen Unfallursachen finden sich auch in den Fahrradstraßen 
in der Art der Konflikte wieder (bspw. Vorfahrtsmissachtung durch kreuzende und einfahrende Fahrzeuge). Dies gilt genauso für die beobachteten Streckenabschnitte. Hier findet sich die Problematik „Parken“ sowohl bei den Unfällen als auch bei den Konflikten.

\subsection{Ergebnisse der Verkehrsteilnehmerbefragung}

Im Rahmen der Verkehrsteilnehmerbefragung in den Fahrradstraßen (Fragebogen siehe Anhang 4) wurden 452 Personen befragt, davon waren zum Zeitpunkt der Befragung 267 (59 \%) als Radfahrer, 122 (27\%) als Autofahrer sowie 63 (14\%) als Fußgänger unterwegs (siehe Abbildung 71).

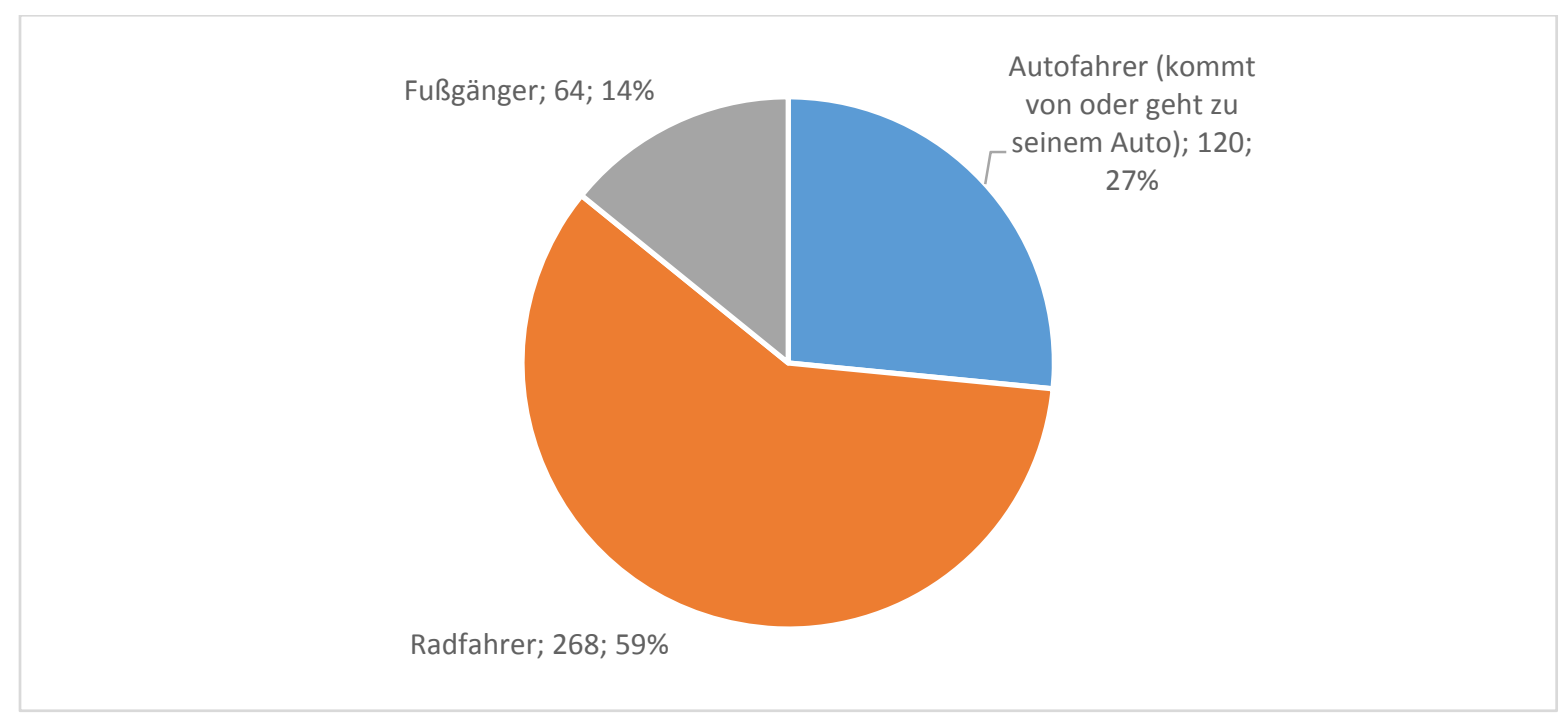

Abbildung 71: Verkehrsmittelnutzung der befragten Personen $(n=452)$

Die größte Gruppe der Befragten (50 \%) stellte die Altersgruppe der 18- bis 49-jährigen dar, weitere 39 \% gehörten zur Altersgruppe der über 50-jährigen, $11 \%$ der Befragten waren unter 18 Jahren alt. Auffällig war, dass in drei Kommunen die unter 18-jährigen bei der Befragung besonders stark vertreten waren. Ein wesentlicher Grund dafür ist wahrscheinlich darin zu sehen, dass die Fahrradstraßen im Zuge von Schulwegen liegen. An der Befragung nahmen etwas mehr Männer (52 \%) als Frauen teil.

\subsubsection{Wissensstand zur Verkehrssicherheit}

Im Rahmen der Befragung war rund einem Viertel (26\%) der befragten Teilnehmer nicht bewusst, dass er sich gerade in einer Fahrradstraße befand, vor allem den Autofahrern (33\%). Den höchsten diesbezüglichen Wert (62\%) wiesen hierbei die Ergebnisse der Befragung in einer Fahrradstraße auf, die ausschließlich beschildert war und bei der auf jede Markierung verzichtet wurde. 
Autos dürfen Radfahrer in Fahrradstraßen überholen.

In Fahrradstraßen gilt auch für Radfahrer das Rechtsfahrgebot.

Die zulässige Höchstgeschwindigkeit beträgt in Fahrradstraßen $30 \mathrm{Km} / \mathrm{h}$.

In Fahrradstraßen dürfen Radfahrer nebeneinander fahren.

An Kreuzungen in Fahrradstraßen hat der Radfahrer nicht immer Vorfahrt

Andere Fahrzeuge dürfen in Fahrradstraßen nur dann fahren, wenn dies ausdrücklich zugelassen ist.

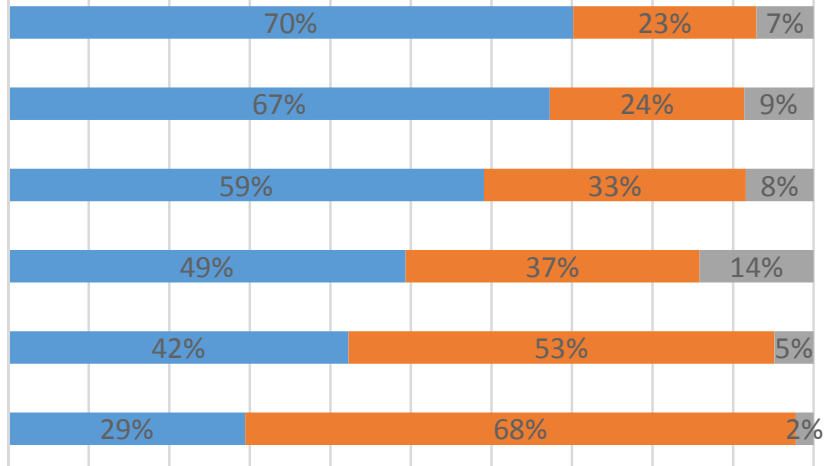

norrekt beantwortet

$\begin{array}{lllllllllll}0 \% & 10 \% & 20 \% & 30 \% & 40 \% & 50 \% & 60 \% & 70 \% & 80 \% & 90 \% & 100 \%\end{array}$

falsch beantwortet $\quad$ weiß nicht

Abbildung 72: Wissensstand zu Fahrradstraßen ( $\quad$ =452 Autofahrer, Radfahrer und Fußgänger)

Autos dürfen Radfahrer in Fahrradstraßen überholen.

In Fahrradstraßen gilt auch für Radfahrer das Rechtsfahrgebot.

Die zulässige Höchstgeschwindigkeit beträgt in Fahrradstraßen $30 \mathrm{Km} / \mathrm{h}$.

In Fahrradstraßen dürfen Radfahrer nebeneinander fahren.

An Kreuzungen in Fahrradstraßen hat der Radfahrer nicht immer Vorfahrt

Andere Fahrzeuge dürfen in Fahrradstraßen nur dann fahren, wenn dies ausdrücklich zugelassen ist.

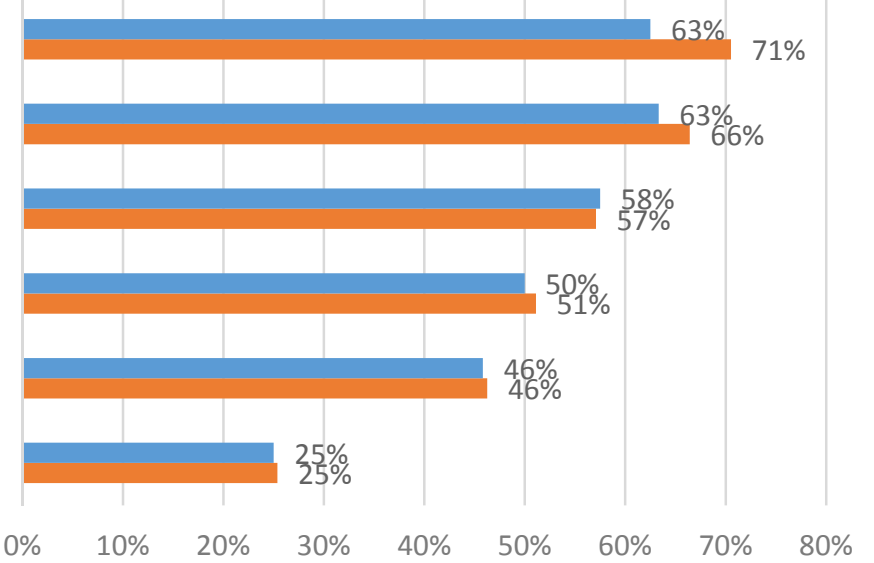

$\begin{array}{lllllllll}0 \% & 10 \% & 20 \% & 30 \% & 40 \% & 50 \% & 60 \% & 70 \% & 80 \%\end{array}$

घutofahrer korrekt beantwortet $\quad$ Radfahrer korrekt beantwortet

Abbildung 73: Wissensstand zu Fahrradstraßen, Vergleich zwischen Autofahrern- $(n=120)$ und Radfahrern $(n=268)$

Der Wissensstand der Teilnehmer der Befragung zu Fahrradstraßen weist mitunter große Lücken auf (Abbildung 72 und Abbildung 73), insbesondere bei den Kfz-Fahrern, bei denen man aufgrund ihres Führerscheinbesitzes voraussetzen sollte, dass sie die geltenden Regeln kennen. So wussten lediglich insgesamt $70 \%$ der Befragten, dass das Überholen von Radfahrern für Kfz in Fahrradstraßen erlaubt ist (Kfz-Fahrer $63 \%$ ). Alle anderen Wissensfragen wurden sogar noch mit deutlich weniger korrekten Aussagen beantwortet. Weniger als ein Drittel der Befragten wussten, dass andere Fahrzeuge in Fahrradstraßen nur dann fahren dürfen, wenn dies ausdrücklich zugelassen ist. Zudem zeigte sich auch, dass sich das Wissen über Fahrradstraßen generell mit den tatsächlichen Begebenheiten vor Ort vermischt. Dies zeigte sich insbesondere bei den Fragen zur Vorfahrtsregelung: Wird bei der Frage zur Vorfahrtsregelung in Fahrradstraßen die tatsächliche Vorfahrtsituation vor Ort berücksichtigt, so steigt der Anteil der korrekten Angaben von $42 \%$ auf $83 \%$. Insgesamt bestehen zwischen den Verkehrsteilnehmergruppen Kfz-Fahrer und Radfahrer nur geringe Unterschiede in der Anzahl der korrekten Antworten. 


\section{Subjektives Sicherheitsempfinden}

Bei der Gesamtheit der Teilnehmer der Befragung überwiegt eine deutlich positive oder zumindest neutrale Gesamtbewertung, wenn sie sich auf einer Fahrradstraße fortbewegen (Abbildung 74). Lediglich 29 \% der Befragten bewerteten die Fahrradstraße, auf der sie gerade unterwegs waren als unsicher, rund 70\% hingegen als sicher. Die Gesamtbewertung der Fahrradstraße variiert jedoch sehr stark nach dem genutzten Verkehrsmittel. Es zeigte sich, dass die befragten Radfahrer (25\%) die Fahrradstraße im Allgemeinen sicherer bewerten als die Kfz-Fahrer (28\%). Auffällig ist die als unsicher geltende Bewertung durch die Fußgänger (48 \%).

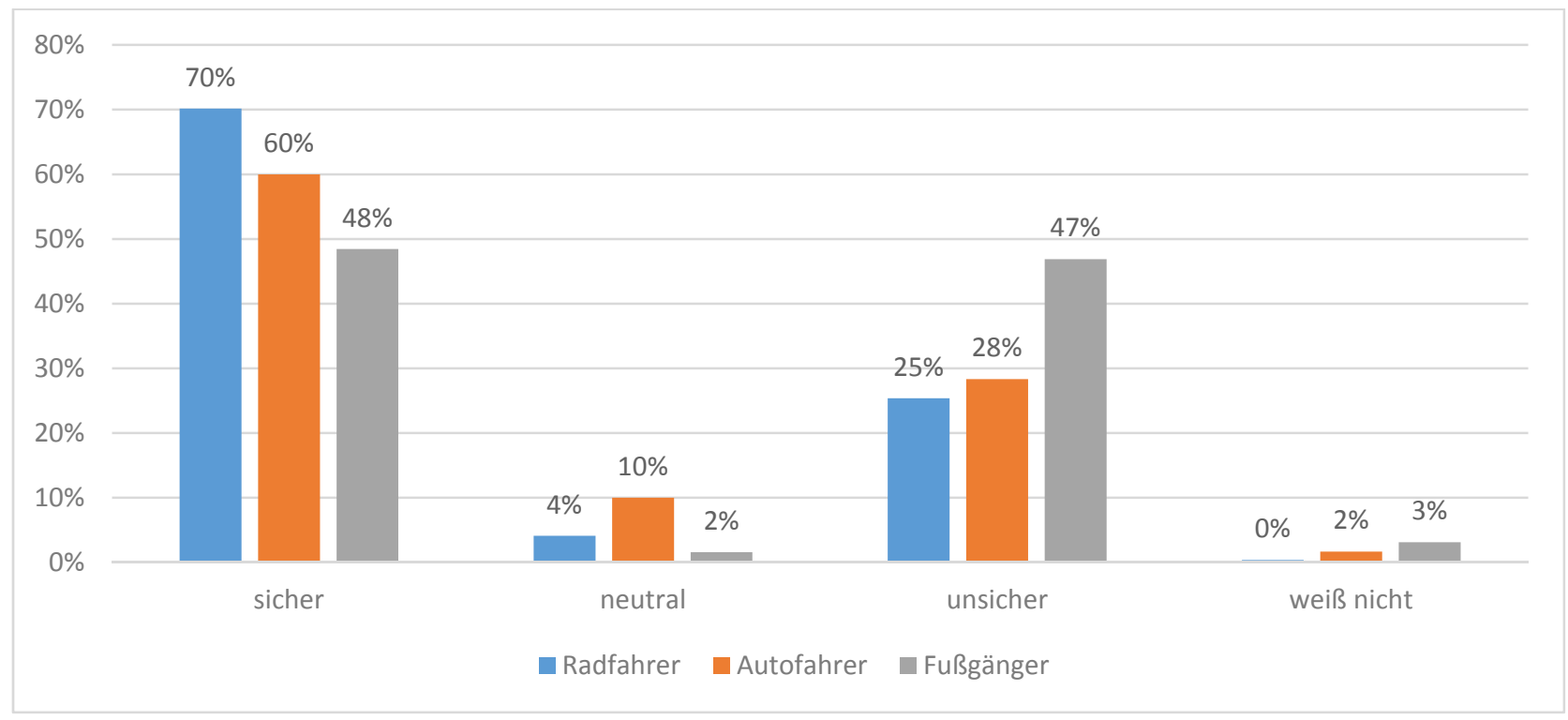

Abbildung 74: $\quad$ Sicherheitsempfinden der Verkehrsteilnehmer nach Art der Verkehrsteilnahme $(n=451)$

Den größten Unsicherheitsfaktor in Fahrradstraßen stellen nach Meinung der Befragten parkende Kfz dar. 51 \% der befragten Radfahrer gaben an, dass diese ihre Fahrt durch die Fahrradstraße unsicherer machen (siehe Abbildung 75). Weitere Unsicherheitsfaktoren stellten die Geschwindigkeit der Autofahrer für $37 \%$ der Radfahrer sowie die Fahrbahn querende Fußgänger für $31 \%$ der Radfahrer dar. Die Gestaltung der Fahrradstraßen wurde lediglich zu $14 \%$ als unsicher wahrgenommen. Am schlechtesten wurde hier eine Fahrradstraße (31 \% unsicher) trotz einer auffälligen Farbmarkierung an der Kreuzung, Fahrradpiktogrammen auf der Fahrbahn sowie einer teilweisen baulichen Umgestaltung bewertet. Hier stellt sich die Frage, ob die Bewertung aufgrund einer Überforderung der Verkehrsteilnehmer durch eine Überfülle an parallelen Markierungen und baulichen Maßnahmen erfolgt ist.

Die Gestaltung von Beginn und Ende der Fahrradstraße wurde von 22 \% der Befragten als unsicher bewertet. Hinsichtlich der Sicherheitsbewertung der Vorfahrtsregelung wurde nach der Art der Vorfahrt unterschieden. Die sicherste Bewertung erhielten die Fahrradstraßen, welche in der Vorfahrt bevorrechtigt sind, was an 7 von 10 Standorten der Fall ist. $80 \%$ bewerteten die Vorfahrtsregelung hier als sicher, bzw. neutral. In der Fahrradstraße mit Rechts-vor-links-Regelung wird die Vorfahrtsregelung zu $68 \%$ als sicher bzw. neutral bewertet. Eine Fahrradstraße ist in der Vorfahrt wartepflichtig, die Art Vorfahrtsregelung bewerteten $63 \%$ als sicher bzw. neutral. Die unsicherste Bewertung erhielt die Fahrradstraße, in deren Verlauf die Art der Vorfahrt zwischen bevorrechtigt und wartepflichtig wechselte, nur $60 \%$ bewerteten die Vorfahrtsregelung dort als sicher bzw. neutral. 


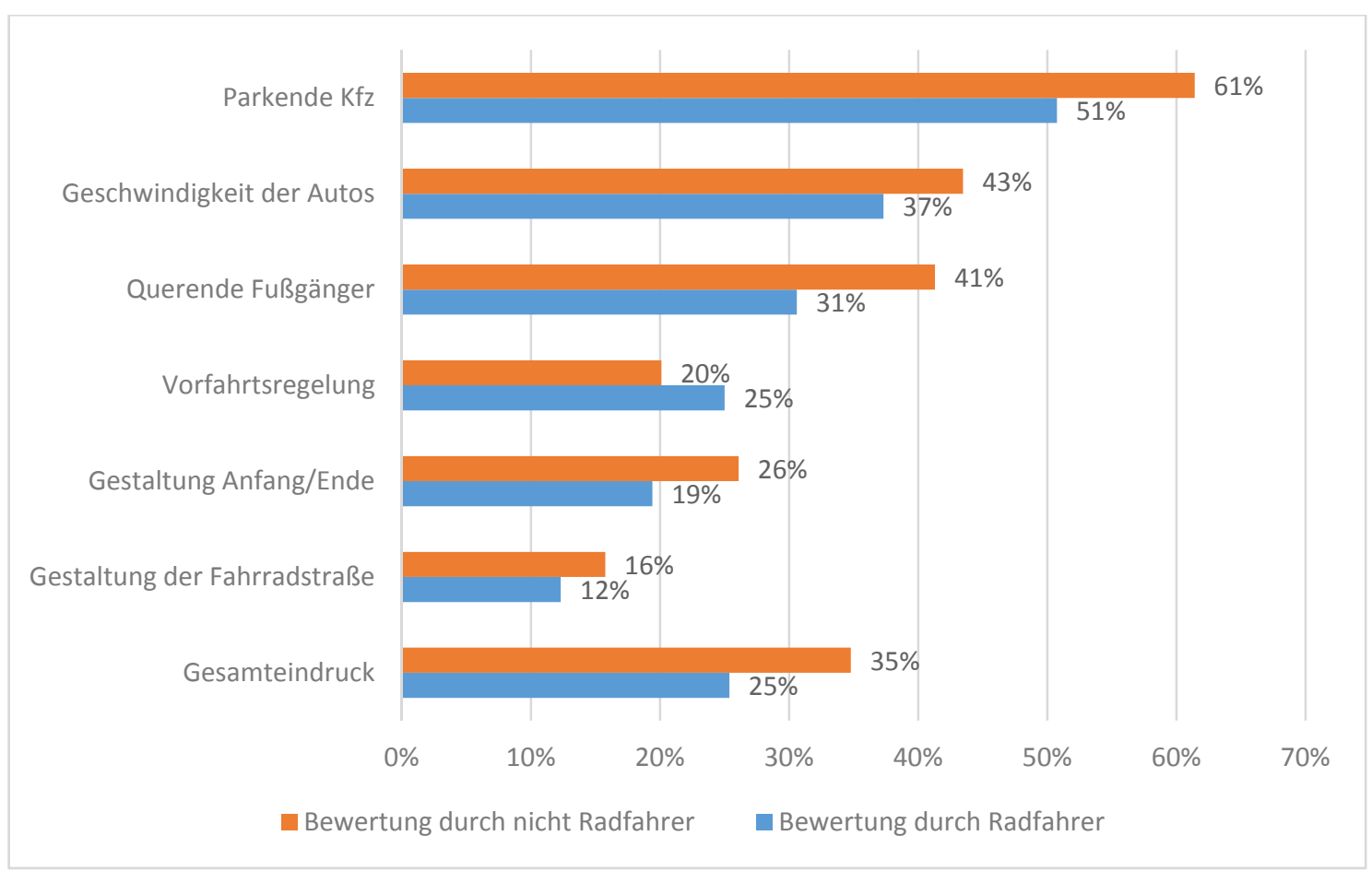

Abbildung 75: Subjektives Sicherheitsgefühl: Anteil „unsicher“ je Aspekt, Vergleich zwischen Radfahrer und sonstigen Verkehrsteilnehmern, $\mathrm{n}=452$

\subsubsection{Zwischenfazit Verkehrsteilnehmerbefragung}

Im Ergebnis zeigt die Verkehrsteilnehmerbefragung, dass der Kenntnisstand bei allen befragten Verkehrsteilnehmern gering ist, erstaunlicherweise bei den $\mathrm{Kfz}$-Fahrern noch geringer als bei den Radfahrern. Die korrekten Antworten lagen in einer Spannweite zwischen $29 \%$ und $70 \%$ je nach Frage. Einem Drittel der befragten Kfz-Fahrer war zudem überhaupt nicht bewusst, dass sie sich gerade auf einer Fahrradstraße befunden haben.

Die Auswertung zum subjektiven Sicherheitsempfinden hat ergeben, dass mit $70 \%$ eine positive bis neutrale Bewertung überwiegt. Als Unsicherheitsfaktoren haben Radfahrer insbesondere die parkenden Pkw wahrgenommen sowie die Geschwindigkeit der fahrenden Kfz und die Fahrbahn querende Fußgänger. Insofern sollte bei der Errichtung von Fahrradstraßen auf diese drei Aspekte besondere Aufmerksamkeit gelegt werden. Insgesamt bewerteten die Radfahrer die Verkehrssicherheit positiver als die anderen Verkehrsteilnehmer.

Trotz des geringen Kenntnisstandes über die Verkehrsregelungen in den Fahrradstraßen werden diese von Radfahrern als sichere Führungsvariante bewertet. So können sich $70 \%$ der befragten Radfahrer und Autofahrer weitere Fahrradstraßen für ihre Stadt vorstellen.

\subsection{Resümee Fahrradstraßen}

Die vorliegende Untersuchung zu Fahrradstraßen sollte erstmals ein Gesamtbild zu Verbreitung, Akzeptanz und Verkehrssicherheit dieser Führungsform in Deutschland vermitteln. Neben der aktuellen Praxis in den Kommunen sollten Auffälligkeiten im Unfallgeschehen, falls vorhanden, beschrieben und Empfehlungen zu deren verkehrssicherer Gestaltung erarbeitet werden.

Zu Verkehrssicherheit von Fahrradstraßen lagen bisher nur wenige wissenschaftlich fundierte Untersuchungen aus den Jahren 1997 und 2003 vor. Sie kommen alle zu ähnlichen Ergebnissen: Die Verkehrssicherheit ist positiv zu bewerten. Allerdings kommt es - ähnlich wie in Tempo 30- 
Zonen - zu Überschreitungen der zulässigen Höchstgeschwindigkeit der Kraftfahrzeuge (Kfz) und im Straßenraum parkende oder haltende $\mathrm{Kfz}$ werden als eine mögliche Konfliktquelle genannt.

Fahrradstraßen werden bundesweit eingesetzt, rund ein Drittel der 359 befragten Kommunen haben sie bereits im Einsatz. Die Wahrnehmung der Fahrradstraßen in den Kommunen ist überwiegend positiv. Konflikte oder Verkehrssicherheitsdefizite werden selten berichtet. Eine bundesweit einheitliche Gestaltung ist nicht festzustellen; allerdings haben einige Kommunen einheitliche Gestaltungvorgaben festgelegt. Das Befahren und das Parken durch Kraftfahrzeuge ist in fast allen Fahrradstraßen erlaubt.

In Fahrradstraßen ist die Unfallschwere geringer als auf Innerortsstraßen generell. Getötete gab es auf den untersuchten Fahrradstraßen im Untersuchungszeitraum nicht. Der Anteil schwerverletzter Verkehrsteilnehmer in den untersuchten Fahrradstraßen (15\%) liegt leicht unter dem bundesweiten innerörtlichen Durchschnitt (17\%) und entspricht in etwa dem Anteil der schwerverletzten Radfahrer auf Nebenstraßen (14\%). Damit liegt die Unfallschwere in Fahrradstraßen in etwa auf dem Niveau von Nebenstraßen und somit unter dem Durchschnitt des innerörtlichen Gesamtnetzes.

Von den 223 untersuchten Fahrradstraßen waren in 42 Straßen gar keine Radverkehrsunfälle zu verzeichnen. Bei näherer (mikroskopischer) Untersuchung der Radverkehrsunfälle wurde zudem deutlich, dass von den 607 gemeldeten Radverkehrsunfällen nur knapp jeder Dritte einen offensichtlichen Zusammenhang mit der Infrastruktur der Fahrradstraße aufweist. Damit verblieben nur noch 75 Fahrradstraßen mit 186 zu untersuchenden Radverkehrsunfällen in der vertiefenden Untersuchung. Nicht relevante Unfälle fanden z.B. auf anliegenden Hauptverkehrsstraßen oder auf Privatgrundstücken statt. Auch Unfälle unter Alkoholeinfluss und Alleinunfälle wurden hier nicht gewertet.

Stellt man die Zahl von 223 untersuchten Fahrradstraßen dem Befund von 186 relevanten Unfällen in einem Untersuchungszeitraum von 5 Jahren gegenüber, wird deutlich, dass Fahrradstraßen sichere Infrastrukturelemente sind.

Im Rahmen der Unfallanalyse wurden alle 186 relevanten Fahrradunfälle nach Strecken- und Knotenunfällen differenziert, um möglichst konkrete Hinweise auf die Handlungsschwerpunkte bei der Gestaltung von Fahrradstraßen zu erhalten.

Die wenigen Unfälle mit Radverkehrsbeteiligung in Fahrradstraßen fanden zu $35 \%$ in den Zwischenknotenpunkten und zu $34 \%$ auf der Strecke statt. Die Unfälle an den Anfangs- und Endknotenpunkten sowie Bushaltestellen und ähnlichen Sondersituationen hatten einen Anteil von $31 \%$. Diese wurden jedoch mikroanalytisch nicht vertieft untersucht, da die sehr unterschiedliche Gestaltung eine Einzelfallanalyse zur Folge gehabt hätte, die keine Aussagen über diesen Typ insgesamt erlaubt hätte. Der Schwerpunkt der Auswertungen lag somit auf den Zwischenknotenpunkten und den Streckenabschnitten.

Bei Unfällen mit Radverkehrsbeteiligung in Fahrradstraßen sind Pkw-Fahrer mit Abstand die häufigsten Unfallgegner (76 \% ohne Alleinunfälle Fahrrad, $66 \%$ mit Alleinunfälle Fahrrad). Danach folgen die Alleinunfälle Fahrrad (13\%) und die Fahrrad/Fahrrad-Unfälle (11\%). Die Bedeutung anderer Verkehrsteilnehmer als Unfallgegner ist gering. Pkw sind nicht nur die häufigsten Unfallgegner, sondern auch die häufigsten Unfallverursacher, sowohl in den Zwischenknotenpunkten (77 \% bei den relevanten Unfällen mit Radverkehrsbeteiligung in der Mikroanalyse) als auch auf der Strecke (75\% bei den relevanten Unfällen mit Radverkehrsbeteiligung in der Mikroanalyse).

Die Analyse von Unfalltyp, Unfallart, Unfallcharakteristik und Unfallursachen hat ergeben, dass an Knotenpunkten Einbiegen-/Kreuzen-Unfälle mit dem Hauptverursacher Kfz dominieren. Die 
Mikroanalyse verdeutlichte, dass sich dahinter vor allem die Missachtung der Vorfahrtsregelungen durch Kfz verbirgt. Die Dominanz der Einbiegen/Kreuzen-Unfälle gibt deutliche Hinweise auf Sicherheitspotenziale für den Radverkehr an Knotenpunkten in Fahrradstraßen. Demnach ist bei der Planung von Fahrradstraßen auf die Zwischenknotenpunkte ein besonderes Augenmerk zu legen.

Unfälle durch ruhenden Verkehr sind die mit Abstand häufigste Unfallursache auf der Strecke und weisen auf Sicherheitsdefizite für den Radverkehr in Bezug auf das Kfz-Parken hin. Durch die mikroskopische Auswertung der Streckenunfälle wurde deutlich, dass das Längsparken für Radfahrer ein Sicherheitsdefizit darstellt, wenn nicht genügend Abstand zwischen Pkw-Tür und Fahrlinie der Radfahrer eingehalten wird, und dass das Ein- und Ausparken in Querparkständen durch nicht ausreichende Sichtverhältnisse auf den fließenden Verkehr für Radfahrer eine Gefahr darstellt.

Auch Überholvorgänge, die durch Pkw vorgenommen werden, sind bei der insgesamt geringen Unfallbelastung eine häufige Unfallkonstellation. Dabei ist das Überholen bei Gegenverkehr in den Unfallkonstellationen am häufigsten zu erkennen.

Die Verhaltensbeobachtung hat in den untersuchten Fahrradstraßen eine geringe Konflikthäufigkeit aufgezeigt. 98,8 \% aller Interaktionen in Fahrradstraßen verliefen konfliktfrei, die meisten Regelverstöße in Knotenpunkten betrafen die Vorfahrtregelung (86 \%). Dies betraf, wie in der Unfallanalyse, unterschiedliche Vorfahrtregelungen. Allerdings finden sich die typischen Unfallursachen auch in der Art der Konflikte wieder, wie Vorfahrtmissachtung durch kreuzende und einfahrende Kfz. Die zulässige Höchstgeschwindigkeit von $30 \mathrm{~km} / \mathrm{h}$ wurde in den beobachteten Fahrradstraßen von den Kraftfahrzeugen nicht immer eingehalten (im Mittel $\mathrm{V}_{85}=36 \mathrm{~km} / \mathrm{h}$ ).

Die Befragung von Verkehrsteilnehmern in Fahrradstraßen hat gezeigt, dass das Wissen über die hier geltenden Verhaltensvorschriften insgesamt sehr lückenhaft ist. Fahrradstraßen werden von $70 \%$ der befragten Verkehrsteilnehmer insgesamt als eine sichere Führungsform genannt. Als Risiken nannten die Befragten die Aspekte „Parkende Kfz" und „Geschwindigkeit der Kfz“. Es besteht also in diesen Aspekten eine Übereinstimmung zwischen den Befunden der Untersuchung und den Einschätzungen der Nutzer.

In der Zusammenschau der Ergebnisse ergibt sich folgendes Bild:

- Fahrradstraßen sind eine verhältnismäßig sichere Führungsform, die von den Verkehrsteilnehmern auch überwiegend als sicher wahrgenommen wird.

- Die spezifischen Gefährdungsmomente sind die Knotenpunkte, zu geringe Abstände zum Parken am Fahrbahnrand und das Überholen. Hier bestehen noch Potenziale zur Erhöhung der Verkehrssicherheit.

- Die Fahrradstraße als Element ist auf der Strecke und bezogen auf die Knotenpunktregelungen zu wenig erkennbar. 


\subsection{Empfehlungen für Fahrradstraßen}

Die Fahrradstraße ist eine sichere Führungsform, die auch bei zum Teil sehr hohem Radverkehrsaufkommen eine geringe Unfallbelastung aufweist. Eine weitere Verbesserung der Sicherheit kann über folgende Handlungsschwerpunkte erreicht werden:

\section{Beschränkung von Kfz-Verkehr}

Fahrradstraßen sind vorrangig Hauptverbindungen für den Fahrradverkehr. Die Zulassung von anderen Verkehrsarten ist sehr sorgfältig zu prüfen. In städtischen Räumen kann der

Anliegerverkehr mit Kfz in der Regel nicht ausgeschlossen werden. Hier ist sicher zu stellen, dass sich im Zuge der Fahrradstraße kein Durchgangsverkehr („Schleichverkehr") entwickelt und der Kfz-Verkehr auf den Anliegerverkehr beschränkt bleibt. Die Zufahrtserlaubnis für Kraftfahrzeuge sollte grundsätzlich auf Anlieger beschränkt sein (Anordnung des Zusatzzeichens StVO 1020-30 „Anlieger frei“), um zusätzliche Probleme mit dem Kfz-Durchgangsverkehr (u.a. vermehrtes Überholen und überhöhte Geschwindigkeiten) zu vermeiden.

\section{Fahrgassenbreiten}

Schmalere Straßen verhindern das Überholen von Radfahrern durch Kfz. Gleichzeitig wird der Seitenabstand von Radfahrern im Begegnungsverkehr zu parkenden Kfz zu gering. Breitere Straßen ermöglichen ein Überholen von Radfahrern, führen aber zu höheren Geschwindigkeiten und begünstigen Überholvorgänge durch $\mathrm{Kfz}$, die eigentlich unterbleiben sollten.

Für Fahrradstraßen werden daher Mindestfahrgassenbreiten empfohlen, um ein sicheres Begegnen zu ermöglichen und Unfälle mit parkenden Kfz zu vermeiden. Die entsprechend notwendigen Fahrgassenbreiten lassen sich aus den erforderlichen Lichtraumprofilen der RASt 2006 ableiten. Um das gleichzeitige Begegnen von jeweils zwei nebeneinander fahrenden Radfahrern sicher zu ermöglichen, sollte die Fahrgasse von Fahrradstraßen im Regelfall $4 \mathrm{~m}$ zuzüglich der notwendigen Sicherheitsabstände zu parkenden Fahrzeugen (beim Längsparken $0,75 \mathrm{~m}$ ) betragen. In diesem Fall sind außerdem ausreichend Sicherheitsabstände zum Überholen eines Radfahrers oder zum Begegnen eines Radfahrers mit einem Pkw vorhanden. Soll gewährleistet werden, dass auch zwei nebeneinander fahrende Radfahrer einem Pkw sicher begegnen können, so ist eine Fahrgassenbreite von mindestens 4,6 m zuzüglich der notwendigen Sicherheitsabstände zu ggf. parkenden Fahrzeugen erforderlich.

Von deutlich größeren Fahrgassenbreiten ist dagegen abzusehen, da sonst überhöhte Geschwindigkeiten der Kraftfahrzeuge aufgrund vermehrter Überholungen provoziert werden können. Nur, wenn das Befahren der Fahrradstraße für Kraftfahrzeuge nicht erlaubt ist oder das Überholen bereits durch hohe Radverkehrsstärken weitgehend unterbunden wird, können auch größere Fahrgassenbreiten Anwendung finden.

\section{Markierung von Sicherheitstrennstreifen}

Die Sicherheitstrennstreifen sollten durch Markierungen gekennzeichnet werden, ggf. unterstützt durch Piktogramme, die den Fahrweg des Radfahrers kennzeichnen. Die Breite der Sicherheitstrennstreifen sollte gemäß den Angaben in den Empfehlungen für Radverkehrsanlagen (ERA) bemessen werden. 


\section{Einheitliche Vorfahrt- und Gestaltungsprinzipien}

In Knotenpunkten ist die Missachtung der Vorfahrt beim Einbiegen oder Kreuzen die häufigste Unfallursache und zugleich der am häufigsten beobachtete Konflikt. Um die Unfallrisiken hier zu vermindern, werden zwei Maßnahmen vorgeschlagen.

Die Vorfahrtregelung im Zuge einer Fahrradstraße sollte einheitlich gestaltet werden. Gemäß der Zweckbestimmung als Hauptroute sollte die Fahrradstraße bevorrechtigt werden. Die Unterordnung der Nebenzufahrten muss dabei jeweils eindeutig erkennbar sein, z.B. durch Beschilderung, Aufpflasterung, abgesenkte Bordsteine, Einengungen o.ä.

Für die Gestaltung von Knotenpunkten sollten einheitliche Gestaltungsprinzipien angewendet werden, die die Situation verdeutlichen. Diese sollen kurzfristig mindestens innerhalb einer Kommune einheitlich sein. Mittelfristig sollten die einheitlichen Gestaltungsvorgaben im technischen Regelwerk bundesweit Anwendung finden.

\section{Überholen durch Kfz vermeiden}

Überholvorgänge durch $\mathrm{Kfz}$ in Fahrradstraßen sollten möglichst unterbleiben. Hier ist zu diskutieren, ob in den Ausführungen der StVO zu Zeichen 244.1 (Fahrradstraßen) ein Überholverbot von Radfahrern durch Kfz verankert werden kann, zumal bereits die heutigen Formulierungen einen weitgehenden Verzicht auf das Überholen nahe legen.

\section{Überwachung und Aufklärung}

Neben der Beachtung der planerischen Aspekte werden polizeiliche Kontrollen zum regelkonformen Verhalten sowie eine entsprechende Aufklärungsarbeit zu den Verkehrsregeln in Fahrradstraßen empfohlen.

\section{Polizeiliche Unfalldatenerhebung}

Die Aufnahme des Infrastrukturelements „Fahrradstraße“ bei der Unfallaufnahme würde die Unfallanalyse und damit ein Monitoring der weiteren Entwicklung deutlich vereinfachen. 


\section{Geöffnete Einbahnstraßen}

\subsection{Literaturauswertung}

\subsubsection{Definitionen, rechtliche Grundlagen und Planungsrichtlinien}

Die rechtlichen Grundlagen für die Einrichtung von geöffneten Einbahnstraßen sind der Straßenverkehrs-Ordnung (StVO) und der Verwaltungsvorschrift zur StVO (VwV-StVO) zu entnehmen. Planungsrichtlinien zu geöffneten Einbahnstraßen finden sich in den Richtlinien zur Anlage von Stadtstraßen (RASt 06). Weitere detaillierte Empfehlungen stehen in den Empfehlungen für Radverkehrsanlagen (ERA 2010).

Nach VwV-StVO, RASt 06 und ERA 2010 (FGSV 2006, FGSV 2010) sollen Einbahnstraßen im Regelfall für den Radverkehr in beide Richtungen geöffnet werden, es sei denn Sicherheitsbedenken sprechen dagegen. Dies ermöglicht eine kontinuierliche Radverkehrsnetzgestaltung. Voraussetzung für die Öffnung einer Einbahnstraße für den Radverkehr ist eine zulässige Höchstgeschwindigkeit von $30 \mathrm{Km} / \mathrm{h}$. Nach der VwV-StVO muss eine ausreichende Begegnungsbreite vorhanden sein, ausgenommen an kurzen Engstellen. Bei Linienbusverkehr oder bei stärkerem Verkehr mit Lastkraftwagen muss die Straßenbreite mehr als 3,50 m betragen. Laut RASt 06 sollte die Regelbreite der Fahrbahn 3,50 m betragen, mindestens jedoch 3,00 m bei ausreichenden Ausweichmöglichkeiten. Bei LKW und Linienverkehr fordert auch die RASt eine Breite von mehr als 3,50 m. Demgegenüber empfiehlt die ERA eine Straßenbreite ab 3,00 m bei ausreichenden Ausweichmöglichkeiten sowie eine Straßenbreite ab 3,50 m bei Linienbusverkehr und/oder hohem LKW-Anteil.

Die Beschilderung erfolgt durch das Zusatzzeichen 1000-33 am Beginn von Einbahnstraßen oder bei Einmündungen auf Einbahnstraßen durch Zusatzzeichen 1000-32 („Radfahrer kreuzen von links und rechts“) zu jedem Zeichen 220 StVO („Einbahnstraße“) und Zusatzzeichen 1022-10 („Radfahrer frei“) zu jedem Zeichen 267 StVO („Verbot der Einfahrt“).

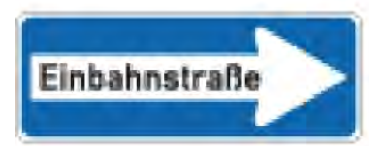

Zeichen 220-20

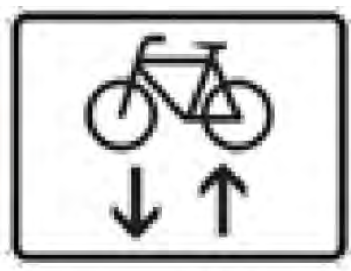

Zeichen 1000-33

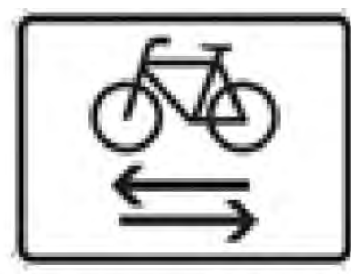

Zeichen 1000-32

Abbildung 76: Beschilderung nach StVO für die Öffnung von Einbahnstraßen für Fahrradgegenverkehr. 


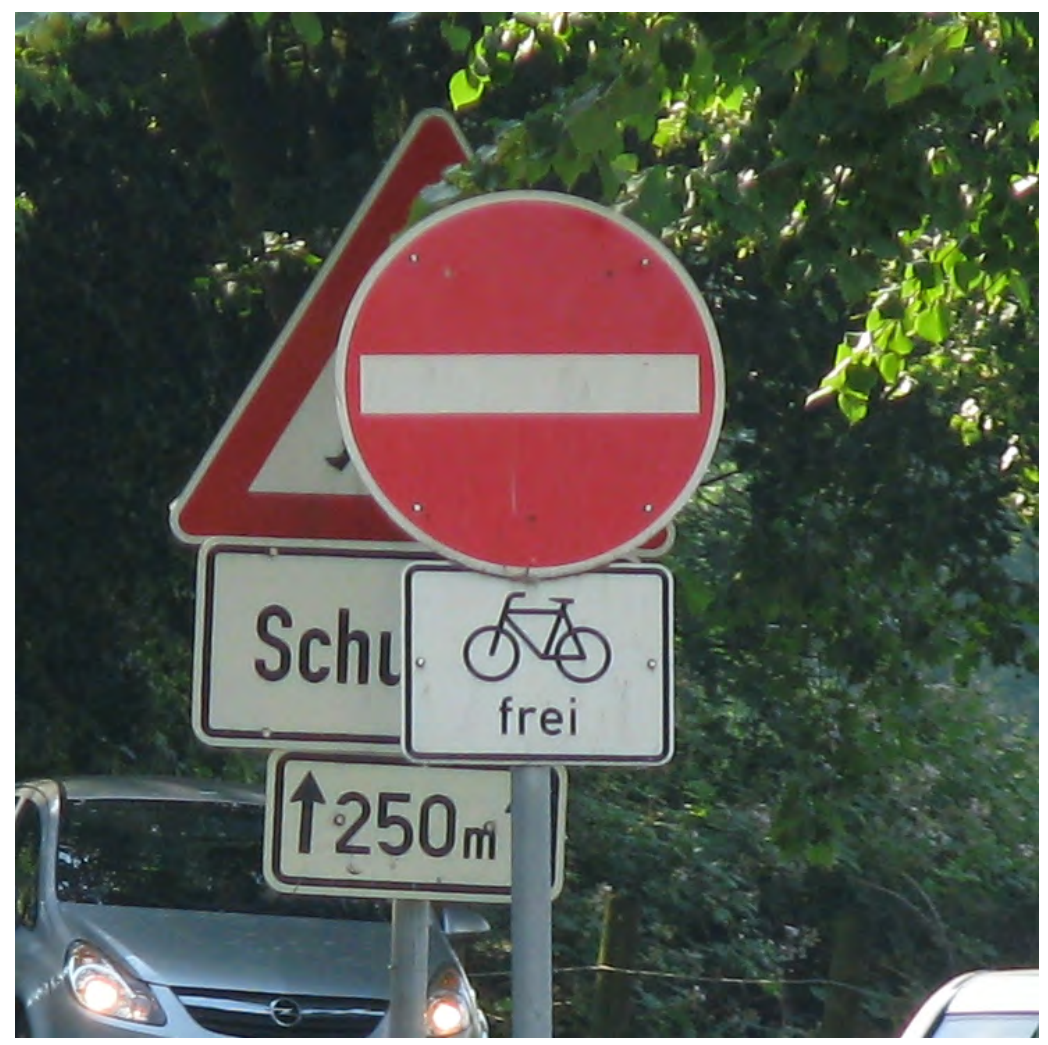

Abbildung 77: Zeichen 267 StVO mit Zusatzzeichen 1022-10.

Bei gefährlichen Rechts-vor-links-Situationen empfiehlt die RASt 06 in kreuzenden Straßen zusätzlich das Zeichen 138 StVO („Radfahrer kreuzen“) mit Zusatzzeichen 1000-30 („beide Richtungen“) anzubringen. Nach den ERA kann zudem in Einzelfällen die Beschilderung durch die Zeichen 102 StVO oder Zeichen 101 StVO mit Zusatzzeichen 1000-32 StVO erfolgen.

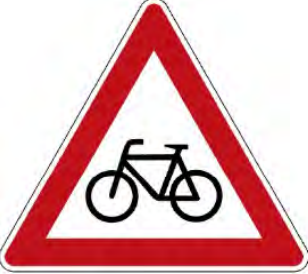

Zeichen $138-10$

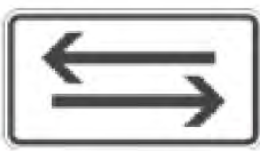

Zeichen $1000-30$

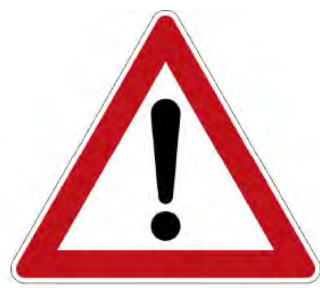

Zeichen 101

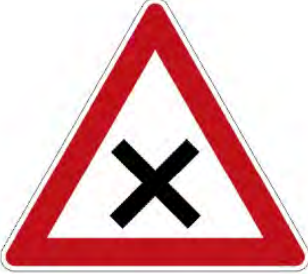

Zeichen 102

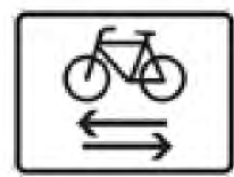

Zeichen 1000-32

Abbildung 78: Mögliche StVO-Beschilderungen an Rechts-vor-links-Kreuzungen.

Wichtige Voraussetzung für die Öffnung einer Einbahnstraße ist laut VwV-StVO das die Verkehrsführung im Streckenverlauf übersichtlich ist, insbesondere an Knotenpunkten und Einmündungen.

Gegebenenfalls ist es orts- und verkehrsbezogen notwendig, einen Schutzraum für den Radverkehr anzulegen (VwV-StVO). Zur Entschärfung möglicher unübersichtlicher Streckenabschnitte oder Knotenpunkte empfiehlt die RASt 06 einen separaten Ein- und Ausfahrtsbereich für den Radverkehr in Gegenrichtung sowie die klare Kennzeichnung der Vorfahrtsregelung an Anschlussknotenpunkten (insb. die Wartepflicht für den Radverkehr in Gegenrichtung). Darüber hinaus bieten sich in Kurvenbereichen Schutz- oder Radfahrstreifen sowie bauliche Maßnahmen an, um zu verhindern, dass Radfahrer geschnitten werden.

Innerhalb von Tempo 30-Zonen ist nach den ERA eine Markierung der Ein- und Ausfahrtsbereiche im Regelfall nicht notwendig. In der Eingewöhnungsphase können jedoch Fahrradpiktogramme mit 
Richtungspfeilen oder Fahrradpforten an Kreuzungen mit Rechts-vor-links-Regelung auf neue Vorfahrtkonstellationen hinweisen. Als weitere Maßnahmen in Kurvenbereichen ergänzt die ERA die Empfehlungen der RASt durch die Wegnahme von Parkständen umso die Sichtbeziehungen zu verbessern sowie die Markierung von Piktogrammen mit Richtungspfeilen. Bei Kreuzungen mit bevorrechtigten Straßen können im Einzelfall auch punktuelle bauliche Maßnahmen wie Auffangradwege oder Fahrbahnteiler sinnvoll sein, wenn die Gefahr besteht, dass schnelle einbiegende $\mathrm{Kfz}$ den wartepflichtigen Radverkehr schneiden oder wartepflichtige $\mathrm{Kfz}$ die Einfahrt für den Radverkehr versperren. In gegenläufiger Richtung ausfahrender Radverkehr sollte auch in die Planung der Kreuzung miteinbezogen werden und durch ein verkleinertes Zeichen 205 StVO („Vorfahrt gewähren“) oder ein Lichtzeichen für den Radverkehr geleitet werden.

Bei Verkehrsstärken über $400 \mathrm{Kfz/h}$ können Schutzstreifen markiert werden. Dafür sind mindestens 3,75 m Fahrbahnbreite notwendig. In Ausnahmefällen kann der Radverkehr auf Radfahrstreifen und Radwegen geführt werden, in Tempo-30-Zonen sind benutzungspflichtige Radverkehrsanlagen aber ausgeschlossen (ERA 2010).

Soll Parken nur auf einer Seite ermöglicht werden, empfiehlt die ERA die in Einbahnfahrtrichtung linke Seite vorzuziehen. Dadurch ergeben sich bessere Ausweichmöglichkeiten für den entgegenkommenden Radverkehr und die Gefahr von sich öffnenden Türen ist durch direkten Sichtkontakt geringer (ERA 2010).

Alternativ zur Öffnung von Einbahnstraßen für den Radverkehr kann überprüft werden, ob die Einbahnregelung aufgehoben werden kann. Das dient in schmalen Straßen auch der Geschwindigkeitsreduktion im Kfz-Verkehr. Ist das Verkehrsaufkommen dafür zu hoch, können „unechte“ Einbahnstraßen umgesetzt werden. In diesen ist der Kfz-Verkehr in beide Richtungen zugelassen, an einem Ende wird aber die Einfahrt durch Zeichen 267 StVO („Verbot der Einfahrt“) verhindert. Auf Hauptverkehrsstraßen, die als Einbahnstraßen ausgewiesen sind und auf denen eine Geschwindigkeitsbeschränkung auf maximal $50 \mathrm{~km} / \mathrm{h}$ gilt, ist eine Zulassung des Radverkehrs in Gegenrichtung nur auf abgetrennten Sonderwegen möglich. Für diese gelten dieselben Anforderungen wie für Zweirichtungsradwege (ERA 2010).

\subsubsection{Verbreitung, Einsatz}

Im Vergleich zu den Fahrradstraßen gibt es eine größere Anzahl an Untersuchungen zum Thema geöffnete Einbahnstraßen. In einer Untersuchung der Bundesanstalt für Straßenwesen (BASt) zur Verkehrssicherheit in Einbahnstraßen mit gegengerichtetem Radverkehr aus dem Jahr 2001 wurde die Verbreitung von verschiedenen Lösungen zur Öffnung von Einbahnstraßen gegen die Kfz-Fahrtrichtung in 200 repräsentativ ausgewählten Kommunen ab 20.000 Einwohnern in Deutschland erhoben. Die konkrete Verbreitung von geöffneten Einbahnstraßen kann aus den veröffentlichten Ergebnissen nicht abgeleitet werden (PGV/ BIS: 2001).

Die Stadt Münster hat 1995 eine ähnlich angelegte Untersuchung zu unechten und echten Einbahnstraßen durchgeführt (STADT MÜNSTER 1995). Neben Querschnittszählungen und einer Unfalldatenanalyse wurden vertiefende Erhebungen an zwei unechten Einbahnstraßen und zum Vergleich einer echten Einbahnstraße vorgenommen. Im Ergebnis belegt die Studie, dass es hinsichtlich des Unfallgeschehens weder in Bezug auf den Gesamtverkehr noch speziell auf den Fahrradverkehr auffällige Unterschiede im Vorher-Nachher-Vergleich gegeben hat. Aufgrund der geringen Fallzahlen ist jedoch eine gesicherte Aussage kaum möglich. Da unechte Einbahnstraßen untersucht wurden und wegen der geringen Fallzahlen in der Untersuchung sind die Ergebnisse für die aktuelle Untersuchung nicht relevant.

1997 erschien bei der BASt eine Studie mit ähnlichem Forschungsdesign zur Sicherheit auf Erschließungsstraßen, bei dem auch geöffnete Einbahnstraßen Gegenstand der Untersuchung waren (ALRUTZ \& STELLMACHER-HEIN: 1997). Im Ergebnis folgern die Autoren, dass die 
Zulassung des Radverkehrs gegen die Einbahnrichtung keine negativen Auswirkungen auf die Verkehrssicherheit hat. Die Erkenntnisse und Erfahrungen dieser Untersuchung flossen in die oben bereits genannte sehr viel breiter angelegte Studie ein, die die BASt über geöffnete Einbahnstraßen im Jahr 2001 vorgenommen hat (PGV/ BIS: 2001).

In PGV/ BIS 2001 erfolgte eine Literaturanalyse zum aktuellen Stand der Öffnung von Einbahnstraßen für den Radverkehr. Danach wurden 200 Städte über 20.000 Einwohner zum Einsatz von geöffneten Einbahnstraßen befragt. Inhaltlich gleichen sich die damalige Erhebung und die hier vorliegende Erhebung mit folgenden Ausnahmen: In der damaligen Untersuchung wurde auch abgefragt, ob Veränderungen im Verkehrsgeschehen auf den Straßen beobachtet und dokumentiert worden sind. Weiterhin wurden ergänzend Verbände zur Regelung von geöffneten Einbahnstraßen befragt. Danach erfolgte eine exemplarische, makroskopische Unfallanalyse für einzelne Bundesländer und Schwerpunktregionen. Als Vergleichsgröße wurden in 15 Städten die Unfälle in der Gesamtstadt ausgewertet, in ausgewählten Einbahnstraßenabschnitten fand zudem eine Vorher-Nachher-Analyse statt. Eine Verkehrsverhaltensanalyse in ausgewählten, geöffneten Einbahnstraßen, die höher mit Rad- und Kfz-Verkehr belastet sind und daher vermutlich mehr Probleme als andere Einbahnstraßen aufweisen, schloss sich an.

Die für die Verkehrssicherheit relevanten Ergebnisse waren dabei:

- Für Fußgänger verringert sich das Gefährdungspotenzial, da sich der Anteil an Radfahrern auf dem Gehweg deutlich reduziert.

- Entgegenkommende Radfahrer und Autofahrer arrangieren sich auch in engen Straßenräumen aufgrund des guten Sichtkontaktes.

- Rund zwei Drittel der Kfz-Fahrer überschritten die zulässige Höchstgeschwindigkeit von $30 \mathrm{~km} / \mathrm{h}\left(\mathrm{V}_{85}=38,9 \mathrm{~km} / \mathrm{h}\right)$.

- Im Begegnungsfall reduzieren die Kfz ihre Geschwindigkeit deutlich.

- Es ergeben sich mehr Konfliktsituationen für Radfahrer, die in Einbahnrichtung fahren als für Radfahrer, die gegen die Einbahnstraße fahren.

- In schmalen geöffneten Einbahnstraßen verliefen die Begegnungen trotz des teilweise geringen Seitenabstandes von 0,43-0,71 m aufgrund des abgestimmten Fahrverhaltens sowie der niedrigen Geschwindigkeiten der Kfz größtenteils unproblematisch.

- In den breiteren Einbahnstraßen führten vermehrt Überholvorgänge seitens der Kfz aufgrund von zu geringen Seitenabständen und zu hohen Geschwindigkeiten zu teilweise kritischen Situationen.

- Es zeigten sich gelegentliche Behinderung durch ein- und ausparkende Fahrzeuge, hauptsächlich für in die Einbahnrichtung fahrende Radfahrer.

- Ein großes Konfliktpotential liegt in Knotenpunktbereichen, insbesondere bei Rechts-vorlinks-Regelung. Es zeigte sich, dass auffallend viele Rad- als auch Kfz- Fahrer eine der Situation nicht angemessene Verhaltensweise zeigten, indem sie den Knotenpunkt mit ungeminderter Geschwindigkeit überfuhren und dies trotz teilweise schlechten Sichtverhältnissen aufgrund parkender Kfz im Knotenpunktbereich. Zudem waren zahlreiche beobachtete Abbiegevorgänge mit einem Schneiden der Kurve verbunden.

- In der Unfallanalyse zeigte sich, dass insgesamt sehr wenige Unfälle mit Radfahrerbeteiligung geschehen.

- Die Unfalldichte ist sogar noch geringer als vor der Öffnung, ebenso zeigte sich eine Verringerung der Unfallschwere. 
Im Ergebnis bestätigt sich die bereits in ALRUTZ \& STELLMACHER-HEIN 1997 gewonnene Einschätzung, dass durch die Öffnung von Einbahnstraßen keine verkehrssicherheitsrelevanten Probleme entstehen, zum Teil konnte sogar ein Sicherheitsgewinn festgestellt werden. Wesentliche Folgerungen und Empfehlungen aus dem Forschungsbericht der BASt (PGV/ BIS 2001) sind:

- Wichtig ist, dass die Öffnung der Einbahnstraßen durch eine umfassende Öffentlichkeitsarbeit begleitet wird.

- Vorhandene Problempunkte lassen sich i.d.R. durch Nachbesserungen nach Einzelfallprüfungen beheben.

- Wesentliche Voraussetzung ist ein niedriges Geschwindigkeitsniveau (zulässige Höchstgeschwindigkeit von $30 \mathrm{~km} / \mathrm{h}$ ).

- Je nach Breite und Kfz-Verkehrsbelastung kommt die Markierung eines Schutzstreifens in Frage sowie die Schaffung von Ausweichmöglichkeiten. Auch Einbahnstraßen mit einer Breite von weniger als 3,00 m können bei entsprechend niedrigem Verkehrsaufkommen und ausreichenden Ausweichmöglichkeiten für den Radverkehr geöffnet werden.

- In Kurvenbereichen sollte generell das Parken verboten werden. Zudem empfiehlt sich eine Markierung von Piktogrammen und eines Schutz- oder Radfahrstreifens, der ggf. durch Poller oder bauliche Trennelemente (z.B.: „Schwellen“) gesichert sein sollte.

- In Knotenpunktbereichen muss ebenfalls für gute Sichtverhältnisse gesorgt werden, durch effiziente Durchsetzung des Parkverbots und gut erkennbare Beschilderung. Eventuell empfehlen sich die Markierung für den gegen gerichteten Radverkehr sowie die bauliche Sicherung der Ein- und Ausfahrt im Übergang zu stark befahrenen Straßen.

Da ALRUTZ \& STELLMACHER-HEIN 1997 nur geöffnete Einbahnstraßen im Zuge von Erschließungsstraßen in Kommunen ab 20.000 EW untersucht haben, sind die damaligen Ergebnisse nur bedingt auf die aktuelle Untersuchung übertragbar. Aber sie wurden als Vergleichsgrundlage für Ergebnisse der aktuellen Untersuchung von problematischen oder unfallträchtigen geöffneten Einbahnstraßen genutzt.

Die Wirksamkeit von geöffneten Einbahnstraßen in Bezug auf die Verkehrssicherheit wurde auch seitens des Kuratoriums für Verkehrssicherheit in Österreich in einer Untersuchung der für den Radverkehr geöffneten Einbahnstraßen in Salzburg analysiert (RETTENBACHER 2000). Die Ergebnisse gleichen denen der deutschen Studie von PGV/ BIS aus dem Jahr 2001. Im Ergebnis zeigt sich, dass Begegnungen zwischen Radfahrern und Kraftfahrzeugen aufgrund des guten Sichtkontaktes unproblematischer verlaufen als Überholvorgänge. Radfahrer, die gegen die Einbahnrichtung fahren, halten einen größeren Abstand zu parkenden Fahrzeugen als jene in gleicher Fahrtrichtung und weichen lediglich im Fall einer Begegnung mit einem Kraftfahrzeug kontrolliert nach rechts aus. Geschwindigkeitsmessungen zeigten, dass Kraftfahrzeuge bei Begegnungen mit Radfahrern deutlich langsamer fahren als bei unbehinderter Fahrt, beim Überholen in gleicher Fahrtrichtung kommt es dagegen zu einer Beschleunigung. Die meisten Konflikte ereignen sich an Knotenpunkten im Zusammenhang mit der Vorfahrt. Als Gestaltungsempfehlung leitet RETTENBACHER eine baulich getrennte Ein- und Ausfahrt für den Radverkehr sowie deutliche Bodenmarkierungen ab, die den Kfz-Fahrern den entgegenkommenden Radverkehr anzeigen sollen.

Weitere Hinweise zur Wahrnehmung der geöffneten Einbahnstraßen durch die Verkehrsteilnehmer lassen sich aus der Begleitforschung zu den Fahrradfreundlichen Städten und Gemeinden in NRW gewinnen (PS \& PGV: 2000). Dort wurden jeweils Befragungen und Verhaltensbeobachtungen durchgeführt, die dem aktuellen Forschungsvorhaben ähnlich sind. Eine vertiefende Untersuchung 
hat insbesondere die Stadt Herford (STADT HERFORD 1997) durchführen lassen: Als Erkenntnis der Untersuchung zeigt sich, dass die Öffnung von Einbahnstraßen für Radfahrer eine erhebliche Verbesserung der Fahrbeziehungen bewirkt und generell zu einer Zunahme des Radverkehrs führt. Kurioserweise wurde aber auch deutlich, dass geöffnete Einbahnstraßen im Vergleich zu den Fahrradstraßen trotz eines damals ebenfalls geringen Bekanntheitsgrades kritischer gesehen werden. Hier sind es vor allem die Gelegenheitsradfahrer, die eine negative Bewertung abgaben, die Gründe dafür bleiben jedoch offen.

\section{Zwischenfazit}

Die bisherigen Veröffentlichungen zum Thema „Für Radverkehr in Gegenrichtung geöffneten Einbahnstraßen“ weisen alle in dieselbe Richtung. Die Öffnung von Einbahnstraßen für den Radverkehr ist problemlos möglich. Besonders wird dies in der Untersuchungen der BASt sichtbar (PGV/ BIS 2001). Es hat sich gezeigt, dass die Öffnung der Einbahnstraßen nicht zu verkehrssicherheitsrelevanten Problemen führt.

\subsection{Ergebnisse der kommunalen Befragung}

\subsubsection{Auswertung der Befragung}

\section{Verbreitung von geöffneten Einbahnstraßen}

Obwohl die Öffnung von Einbahnstraßen für den Radverkehr seit 1997 möglich ist, wurde bis heute nicht erhoben, wie weit verbreitet dieses Infrastrukturelement eingesetzt wird. Im Rahmen der vorliegenden Untersuchung zeigte sich, dass 260 der 359 befragten Kommunen (83 \%) Einbahnstraßen für den Radverkehr in Gegenrichtung freigegeben haben. Die Kommunen, welche an der Befragung teilgenommen haben, verteilen sich dabei über das gesamte Bundesgebiet. (Abbildung 79). 


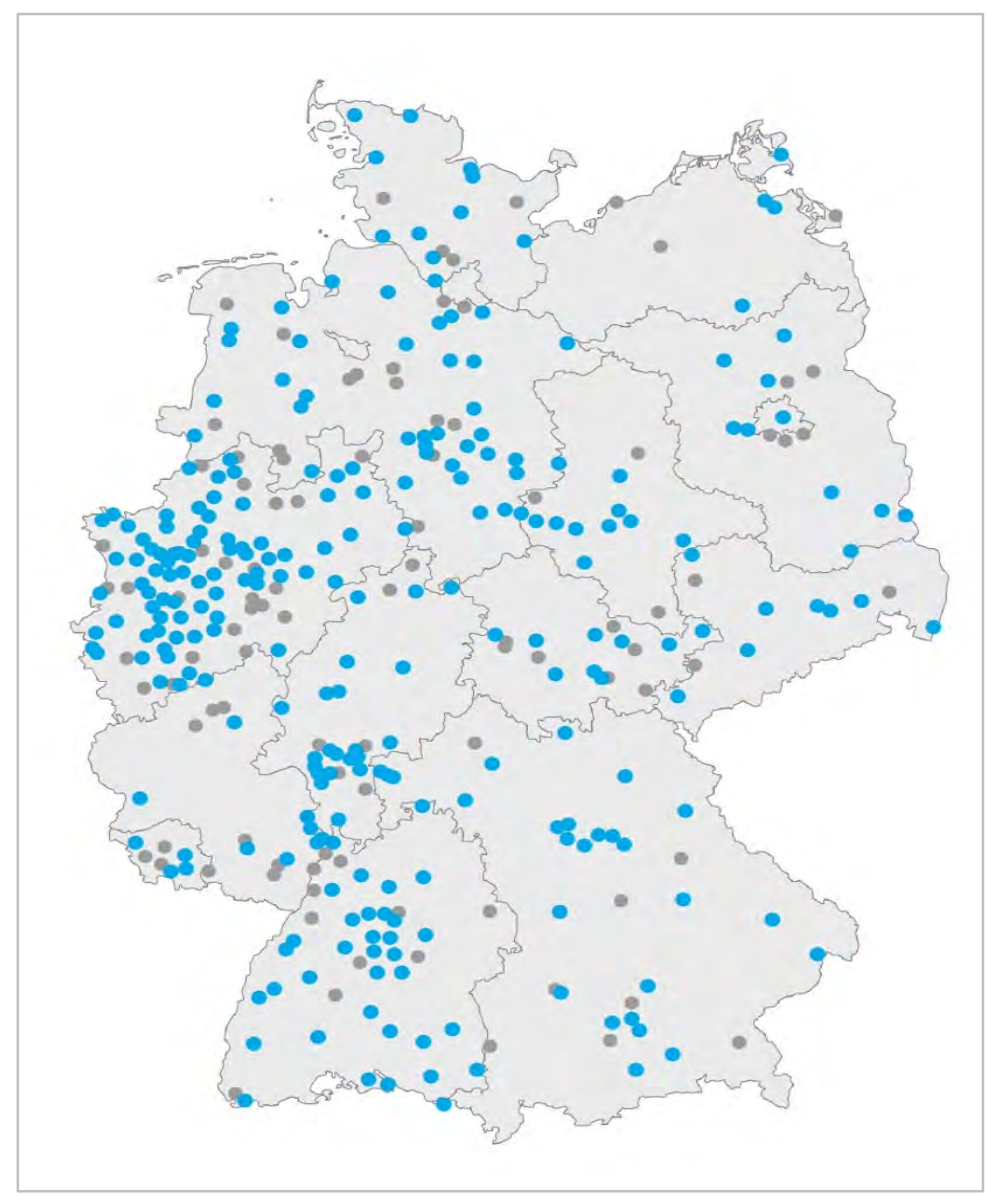

Kommunen mit geöffneten Einbahnstraßen, die teilgenommen haben.

Alle weiteren Kommunen ohne geöffnete Einbahnstraßen, die teilgenommen haben.

Abbildung 79: Räumliche Verteilung der Kommunen mit /ohne geöffnete Einbahnstraßen ( $n=260 / n=99)$

Insgesamt haben 14 Kommunen angegeben, keine Einbahnstraßen zu haben. In der kleinsten Kommunenklasse bis 10.000 Einwohnern kommt dies mit $13 \%$ am häufigsten vor, gefolgt von der Größenklasse bis 20.000 Einwohnern (9\%) und der Klasse bis 50.000 Einwohnern mit $3 \%$. Alle weiteren Städte mit mehr als 50.000 Einwohnern weisen Einbahnstraßen auf.

Alle befragten Kommunen mit mehr als 50.000 Einwohnern haben Einbahnstraßen für den Radverkehr in Gegenrichtung geöffnet. In den Klassen zwischen 10.000 und 50.000 Einwohnern sind von 181 Kommunen mit Einbahnstraßen in 82 \% (148) der Kommunen Einbahnstraßen geöffnet. Lediglich in den 17 Kommunen mit weniger als 10.000 Einwohnern, die Einbahnstraßen haben, sind geöffnete Einbahnstraßen mit 35 \% (6) vergleichsweise selten (Abbildung 80).

Insgesamt haben 260 Kommunen angegeben, 2.373 Einbahnstraßen geöffnet zu haben.

Die meisten Kommunen (57 \%) nutzen Pressemitteilungen, um die Öffnung von Einbahnstraßen für den Radverkehr publik zu machen, gefolgt von stadtweiten allgemeinen Informationen (15 \%). In rund ein Fünftel der Kommunen (22 \%) mit geöffneten Einbahnstraßen fand gar keine Öffentlichkeitsarbeit statt. 


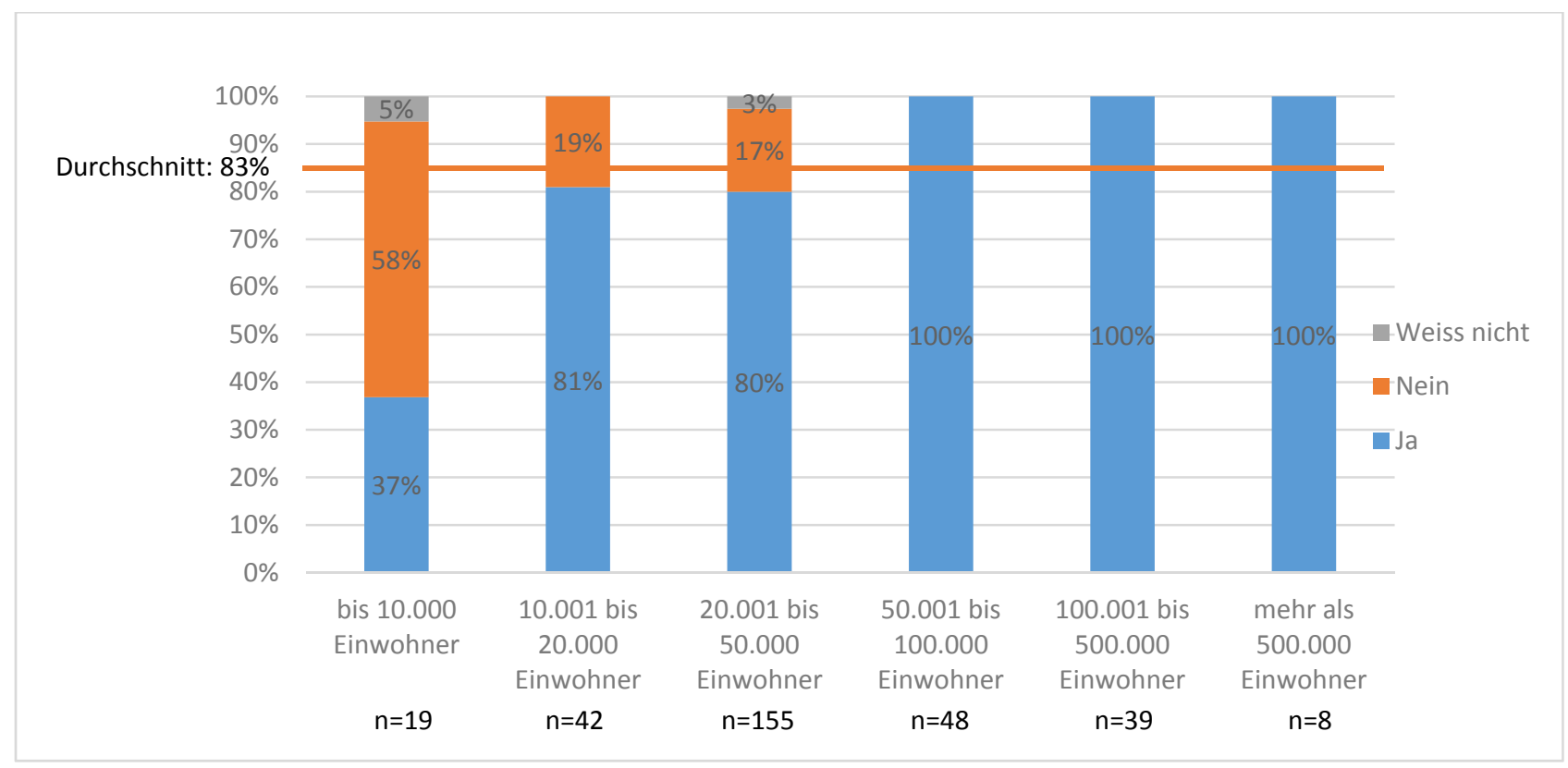

Abbildung 80: Kommunen mit geöffneten Einbahnstraßen nach Kommunengröße $(n=311)$

\section{Einschätzung der Verkehrssicherheit in geöffneten Einbahnstraßen}

Lediglich 25 der 2.373 gemeldeten geöffneten Einbahnstraßen (1 \%) wurden von den Kommunen im Rahmen der Befragung in Phase 1 als problematisch/unfallträchtig eingeschätzt. Dies ist ein sehr positives Ergebnis. Da es jedoch auf der subjektiven Einschätzung der kommunalen Mitarbeiter beruht, musste dieser Eindruck erst noch in der Unfallanalyse überprüft werden. Bei der Öffnung von Einbahnstraßen für den Radverkehr in Gegenrichtung fand im Vergleich zu den Fahrradstraßen mit $25 \%$ deutlich häufiger eine Vorher-/Nachher-Untersuchung statt. In zehn Kommunen wurde die Öffnung von Einbahnstraßen schon einmal wieder rückgängig gemacht, davon in sechs Kommunen aufgrund unzureichender Verkehrssicherheit.

\section{Wahrnehmung von geöffneten Einbahnstraßen}

Für die als problematisch eingestuften geöffneten Einbahnstraßen wurde erhoben, ob Beschwerden aus der Bevölkerung vorliegen. Dabei handelt es sich um eine sehr kleine Stichprobe von 17 geöffneten Einbahnstraßen, von denen in lediglich drei Straßen Bedenken aus der Bevölkerung geäußert wurden. Dies waren:

- "Radweg wird zugeparkt $\rightarrow$ Radfahrer müssen dann auf Fahrbahn ausweichen (gefährlich)“

- „Anlieger sowie Radfahrer machten auf Gefährdungslage aufmerksam“

- „Anlieger befürchteten Gefährdung für den Autofahrer“.

Es zeigt sich bei der Wahrnehmung der Öffnung von Einbahnstraßen durch die Bevölkerung ein ähnliches Bild wie bei den Kommunen: In den 17 durch Kommunen als problematisch eingestuften geöffneten Einbahnstraßen wurden von der Bevölkerung nur in geringem Maß (3 Fälle) ebenfalls sicherheitsrelevante Anmerkungen eingebracht.

\section{Gestaltung von geöffneten Einbahnstraßen}

Bei der Öffnung der 17 von den Kommunen als problematisch bewerteten Einbahnstraßen wurde eine breite Kombination von Maßnahmen eingesetzt. Obligatorisch ist die Beschilderung nach StVO (100 \%). In mehr als der Hälfte der Straßen (in 9 von 17) wurden zusätzlich Markierungen eingesetzt: 
- Zeichen 240,

- Piktogramm „Fahrrad mit Richtungspfeilen“,

- Markierungen in den Einfahrtbereichen und einmündenden Straßen,

- in Einfahrt- und Endknotenpunkten sind dies markierte Einfädelhilfen für den Radverkehr

- und die Anlage von kurzen Auffangschutzstreifen.

In sechs der 17 Straßen wird der Radverkehr über Schutz- bzw. Radfahrstreifen gegen die Fahrtrichtung geführt. Darüber hinaus wurden auch in drei Straßen, in denen der Radverkehr im Mischverkehr auf der Fahrbahn geführt wird, Markierungen (Piktogramme mit und ohne Richtungspfeilen) eingesetzt. Eine bauliche Umgestaltung der Straße oder die Neuordnung des Kfz-Parkens wurde in jeweils fünf Straßen umgesetzt. In zwei Straßen mit Radfahrstreifen wurden alle vier Maßnahmen realisiert.

Die Gestaltung der geöffneten Einbahnstraßen wird in der Mehrheit nach den Vorschlägen der Empfehlungen für Radverkehrsanlagen (ERA) (Schutzstreifen in Gegenrichtung, Markierung von Piktogrammen und Richtungspfeilen im Verlauf, Einfahrtshilfen) vorgenommen (vgl. Kapitel 4.1). Nur in vier der 17 Straßen wurden keine weiteren Maßnahmen außer der Beschilderung eingesetzt.

\subsubsection{Zwischenfazit aus der kommunalen Befragung}

Die Öffnung von Einbahnstraßen ist ein verbreitetes Infrastrukturelement zur Führung des Radverkehrs. Es wird in Kommunen ab einer Ortsgröße von mehr als 10.000 Einwohnern bundesweit in mehr als $80 \%$ aller Kommunen eingesetzt. In Gemeinden unter 10.000 Einwohnern werden seltener Einbahnstraßen geöffnet (in rund einem Drittel der Kommunen), hier sind aber auch seltener Einbahnstraßen überhaupt vorhanden.

Trotz ihrer großen Anzahl werden geöffnete Einbahnstraßen von den kommunalen Verwaltungen kaum als auffällig in Bezug auf die Verkehrssicherheit wahrgenommen. Die geringe Anzahl der Beschwerden seitens der Bevölkerung weist in die gleiche Richtung.

Die Gestaltung der 17 als problematisch gemeldeten geöffneten Einbahnstraßen variiert. Neben der obligatorischen Beschilderung sind in rund der Hälfte zusätzlich Markierungen (Zeichen 240, Fahrradpiktogramme, Markierungen in den Einfahrtbereichen, sowie Einfädelhilfen und Schutzstreifen für den Radverkehr) aufgebracht, in rund einem Drittel wird der gegenläufige Radverkehr auf einem Schutz- oder Radfahrstreifen geführt. Ebenfalls in rund einem Drittel wurde bei Einrichtung der geöffneten Einbahnstraßen das Parken neu geordnet oder wurden bauliche Maßnahmen realisiert.

\subsection{Unfallanalyse geöffnete Einbahnstraßen}

In diesem Kapitel werden die Unfalldaten der untersuchten geöffneten Einbahnstraßen zu Unfallkategorie, Unfalltyp, Unfallart, Unfallort, Unfallbeteiligung und Unfallursachen ausgewertet und die Ergebnisse dargestellt. Andere Parameter wie soziodemografische Daten wurden überprüft. Da diese Ergebnisse unauffällig waren, wurde auf eine Darstellung verzichtet.

Eine detaillierte Übersicht über die Zusammensetzung der Stichprobe in den einzelnen Analyseschritten wurde bereits in Kapitel 2.3.2 gegeben.

\subsubsection{Unfallschwere}

Die Unfälle mit Radfahrerbeteiligung in Einbahnstraßen haben deutlich weniger schwere Unfallfolgen als im bundesweiten Durchschnitt (Abbildung 81). Bei den untersuchten 54 Unfällen in fünf Jahren gab es zwei schwerverletzte Personen. Damit ist die Wahrscheinlichkeit, dass Radfahrer in geöffneten Einbahnstraßen bei einem Unfall schwer verletzt werden, sehr gering. 


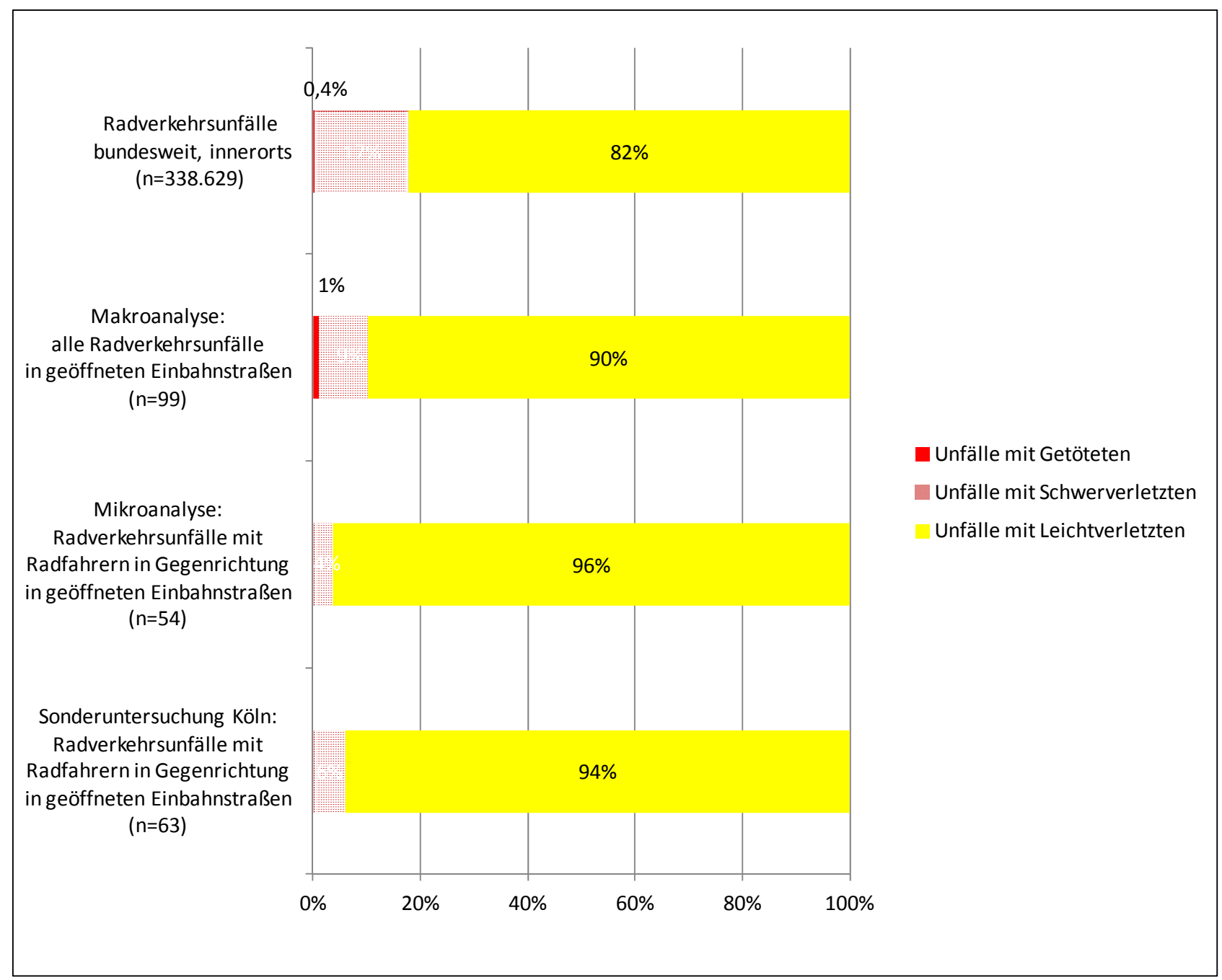

Abbildung 81: Vergleich der Unfallkategorie in geöffneten Einbahnstraßen in den einzelnen Analyseschritten mit den bundesweiten Innerortsunfällen $(U(P), 2008-2012)^{43}$

Wird die Unfallkategorie nach Unfallort, unterschieden in Zwischenknotenpunkt und Strecke, analysiert, ergibt sich folgendes Bild (siehe Abbildung 82):

43 Quelle Unfälle bundesweit, innerorts: Statistisches Bundesamt (2009-2013): Fachserie 8, Reihe 7, 20082012 
Mikroanalyse:

Radverkehrsunfälle mit Radfahrern in Gegenrichtung in geöffneten Einbahnstraßen in Zwischenknotenpunkten ( $n=22)$

Mikroanalyse:

Radverkehrsunfälle mit Radfahrern in Gegenrichtung in geöffneten Einbahnstraßen auf der Strecke $(n=15)$

Sonderuntersuchung Köln: Radverkehrsunfälle mit Radfahrern in Gegenrichtung in geöffneten Einbahnstraßen in Zwischenknotenpunkten $(n=19)$

Sonderuntersuchung Köln: Radverkehrsunfälle mit Radfahrern in Gegenrichtung in geöffneten Einbahnstraßen auf der Strecke $(n=27)$

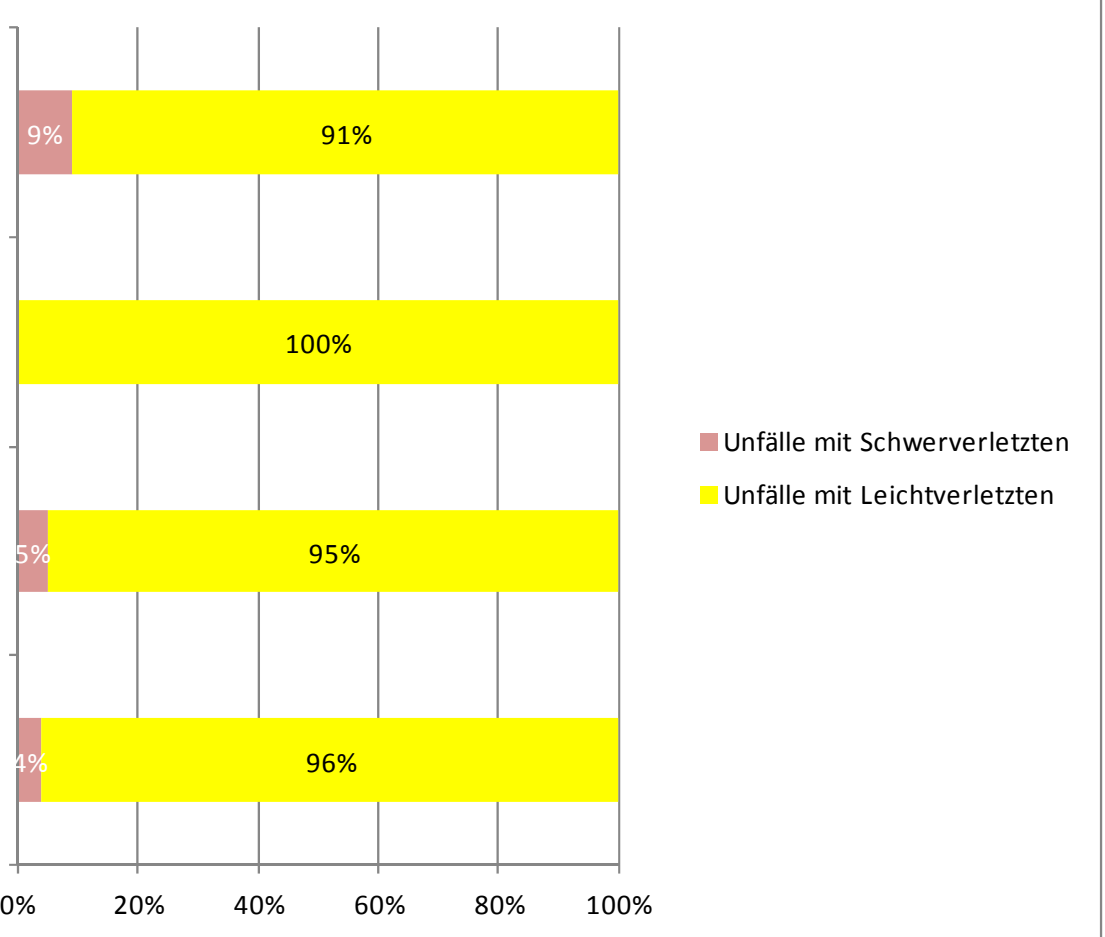

Abbildung 82: Vergleich der Unfallkategorie in geöffneten Einbahnstraßen in Zwischenknotenpunkten und auf der Strecke in der Mikroanalyse und in der Sonderuntersuchung

In den bundesweit untersuchten 17 geöffneten Einbahnstraßen werden Radverkehrsunfälle mit Schwerverletzten vor allem in Knotenpunkten registriert. In der Sonderuntersuchung der Kölner Innenstadt finden die wenigen zwischen 2008 und 2012 registrierten relevanten Unfälle mit Schwerverletzten in geöffneten Einbahnstraßen sowohl im Knotenpunkt als auch auf der Strecke statt. 


\subsubsection{Unfalltyp}

Die Einbiegen/Kreuzen-Unfälle sind in geöffneten Einbahnstraßen bei den Unfällen mit Radfahrern, die in Gegenrichtung fahren, der mit Abstand dominante Unfalltyp. Aber auch Unfälle im Längsverkehr finden in geöffneten Einbahnstraßen im Vergleich zu den bundesweit registrierten Radverkehrsunfällen auf Innerortsstraßen deutlich häufiger statt (siehe Abbildung 83).

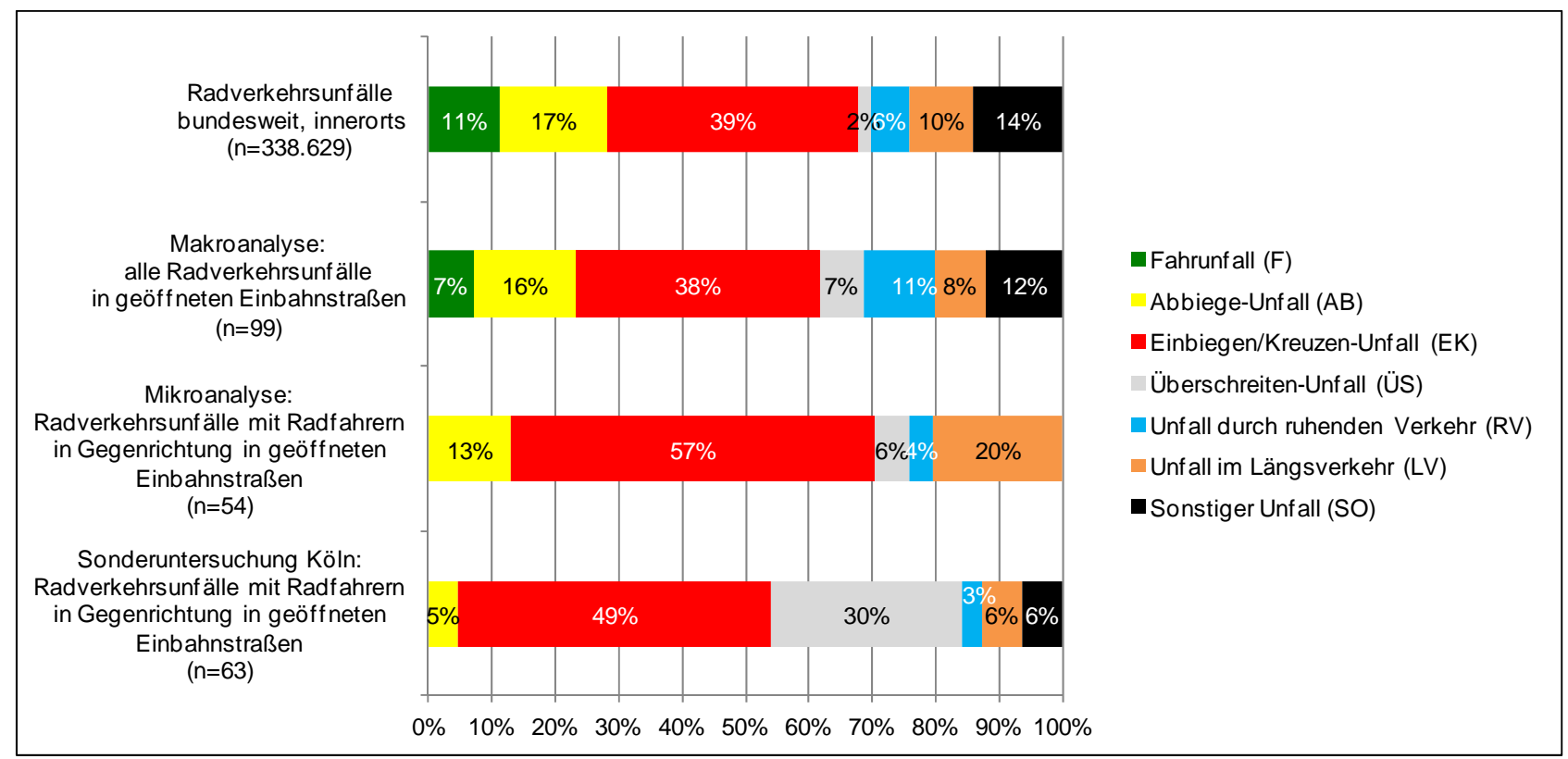

Abbildung 83: Vergleich des Unfalltyps in geöffneten Einbahnstraßen in den Analysephasen mit den bundesweiten Innerortsunfällen $(U(P), 2008-2012)^{44}$

Finden in den bundesweit untersuchten 17 geöffneten Einbahnstraßen schon mehr ÜberschreitenUnfälle statt als im Durchschnitt der Radverkehrsunfälle innerorts, so ist dieser Typ in der Sonderuntersuchung zu den Kölner Einbahnstraßen sogar nochmal deutlich häufiger vertreten.

Abbildung 84 zeigt, dass sich der Unfalltyp bei Unfällen, an denen Radfahrer beteiligt sind, die gegen die Einbahnrichtung fahren, im Hinblick auf den Unfallort Strecke oder Zwischenknotenpunkt wesentlich unterscheidet.

44 Quelle Unfälle bundesweit, innerorts: Statistisches Bundesamt (2009-2013): Fachserie 8, Reihe 7, 20082012 


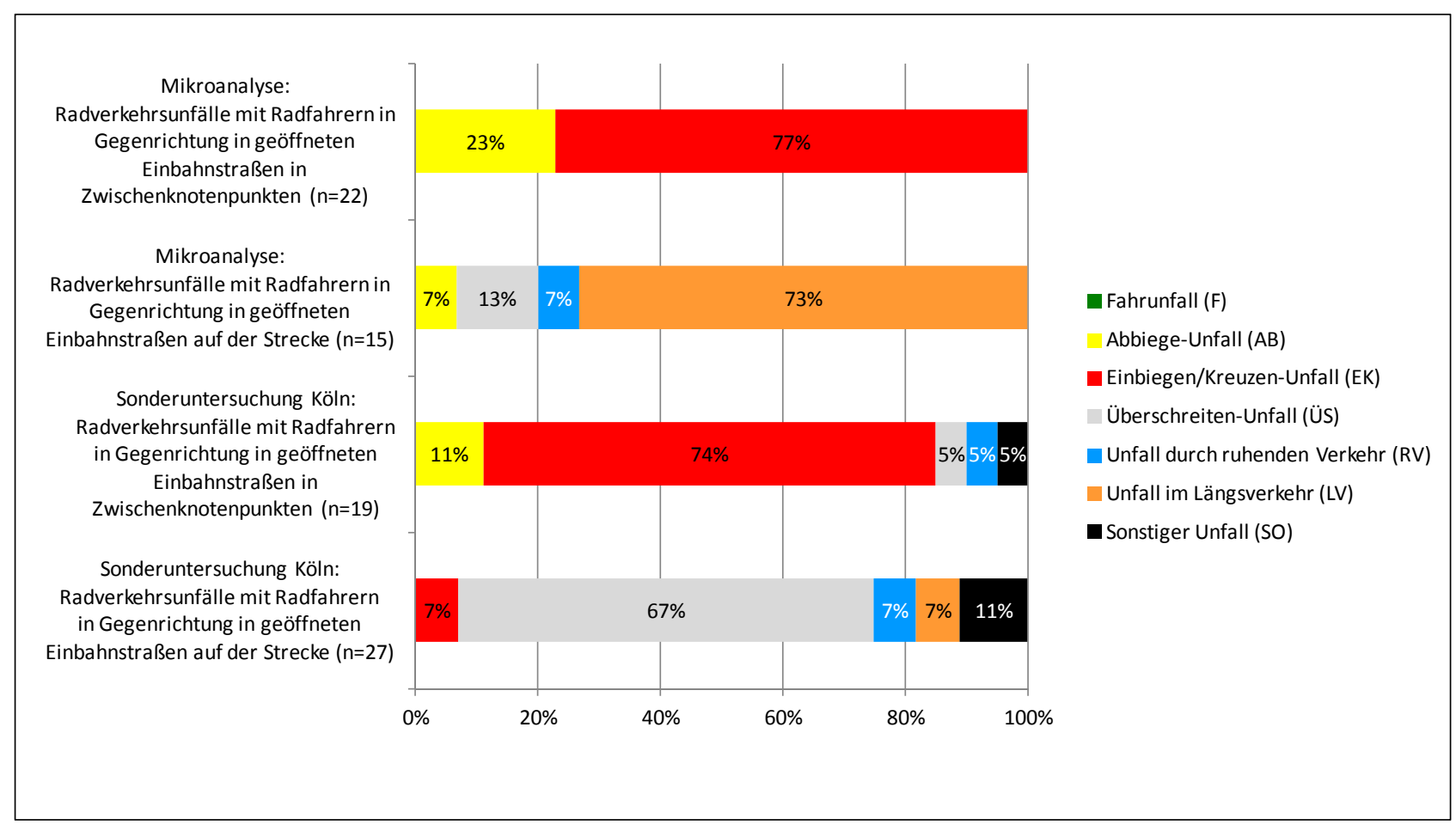

Abbildung 84: Vergleich des Unfalltyps in geöffneten Einbahnstraßen in Zwischenknotenpunkten und auf der Strecke

In den Zwischenknotenpunkten finden in den bundesweit untersuchten 17 geöffneten Einbahnstraßen ausschließlich Einbiegen/Kreuzen-Unfälle und Abbiege-Unfälle statt. Auf der Strecke dagegen dominieren die Unfälle im Längsverkehr.

Im Vergleich der Ergebnisse in den bundesweit untersuchten 17 geöffneten Einbahnstraßen mit denen der Kölner Innenstadt, sind vor allem die Unterschiede auf der Strecke augenfällig: In der Kölner Innenstadt finden wesentlich mehr Überschreiten-Unfälle statt, die Unfälle im Längsverkehr weisen nur einen geringen Anteil auf.

\section{Unfallort in der Sonderuntersuchung zu den geöffneten Einbahnstraßen in der Kölner Innenstadt}

Da sich die Ergebnisse der Sonderuntersuchung zu den geöffneten Einbahnstraßen in der Kölner Innenstadt deutlich von denen in den bundesweit untersuchten 17 geöffneten Einbahnstraßen unterscheiden, wurden die Kölner Unfälle verortet, um die geöffneten Einbahnstraßen, die mit Unfällen belegt waren, detailliert untersuchen zu können (siehe Abbildung 85). 


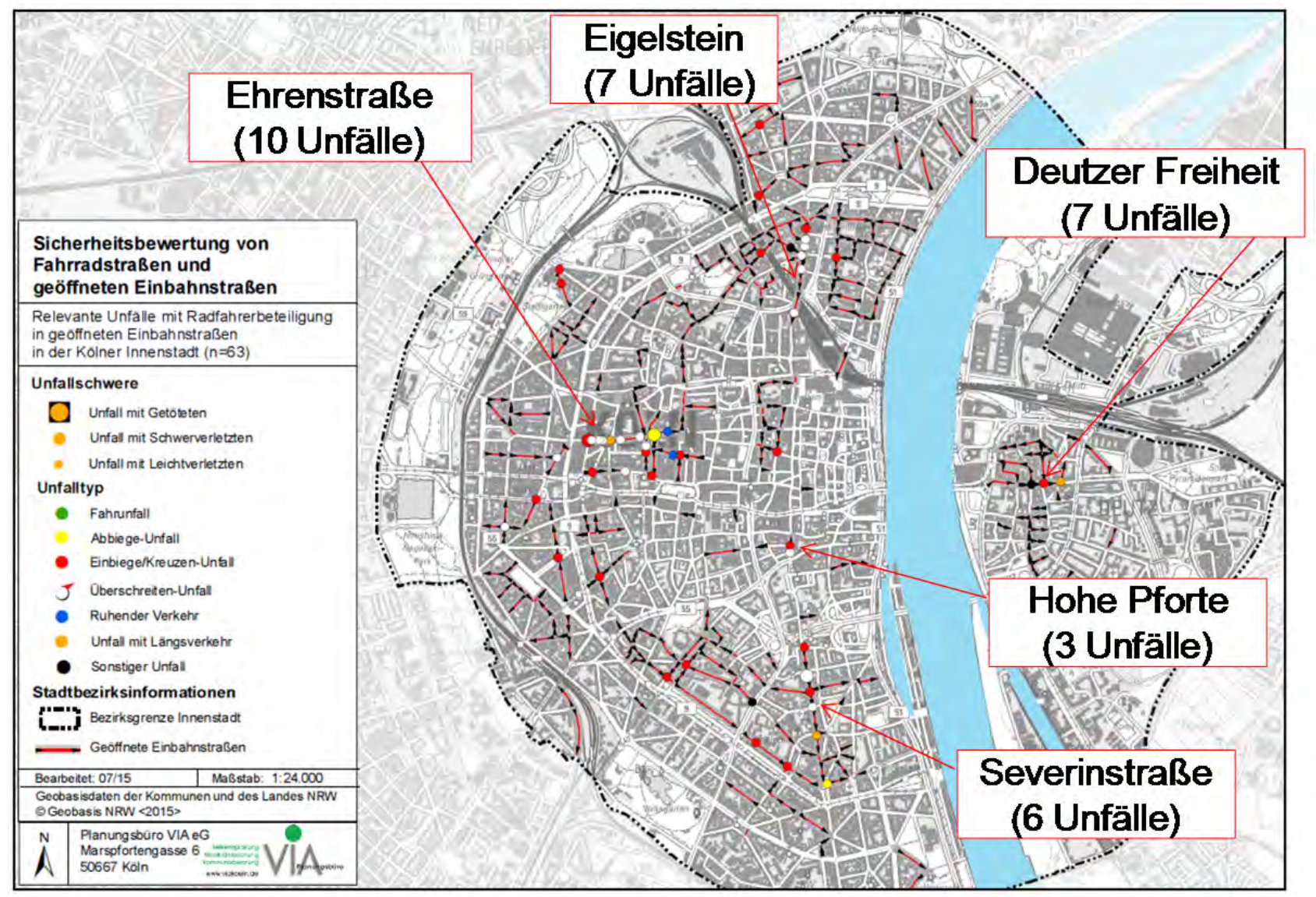

Abbildung 85: Verortung der relevanten Unfälle mit Radverkehrsbeteiligung in geöffneten Einbahnstraßen in der Kölner Innenstadt nach Unfallkategorie und Unfalltyp

In der untersuchten Kölner Innenstadt fanden im betrachteten Untersuchungszeitraum 63 Unfälle in geöffneten Einbahnstraßen statt, bei denen Radfahrer entgegen der Einbahnrichtung fuhren. Das sind durchschnittlich nur knapp 13 Unfälle pro Jahr in insgesamt 201 geöffneten Einbahnstraßen. Von diesen 63 Unfällen geschahen 33 Unfälle (52 \%) in den fünf Haupteinkaufsstraßen in der Kölner Innenstadt. 13 Unfälle sind Überschreiten-Unfälle in diesen fünf Straßen, d.h. Fußgänger achteten beim Überqueren der Straße nicht auf Radfahrer, die gegen die Einbahnstraßenrichtung fuhren. 


\subsubsection{Unfallart}

Die Auswertungen zur Unfallart bestätigen nur zum Teil die Ergebnisse zum Unfalltyp: In geöffneten Einbahnstraßen finden mehr Unfälle der Unfallart Zusammenstoß zwischen Fahrzeug und Fußgänger statt als im Durchschnitt der bundesweiten innerörtlichen Unfälle mit Radverkehrsbeteiligung. Die häufige Registrierung dieser Unfallart deutet erneut auf Konflikte zwischen Radfahrern und Fußgängern hin (siehe Abbildung 86).

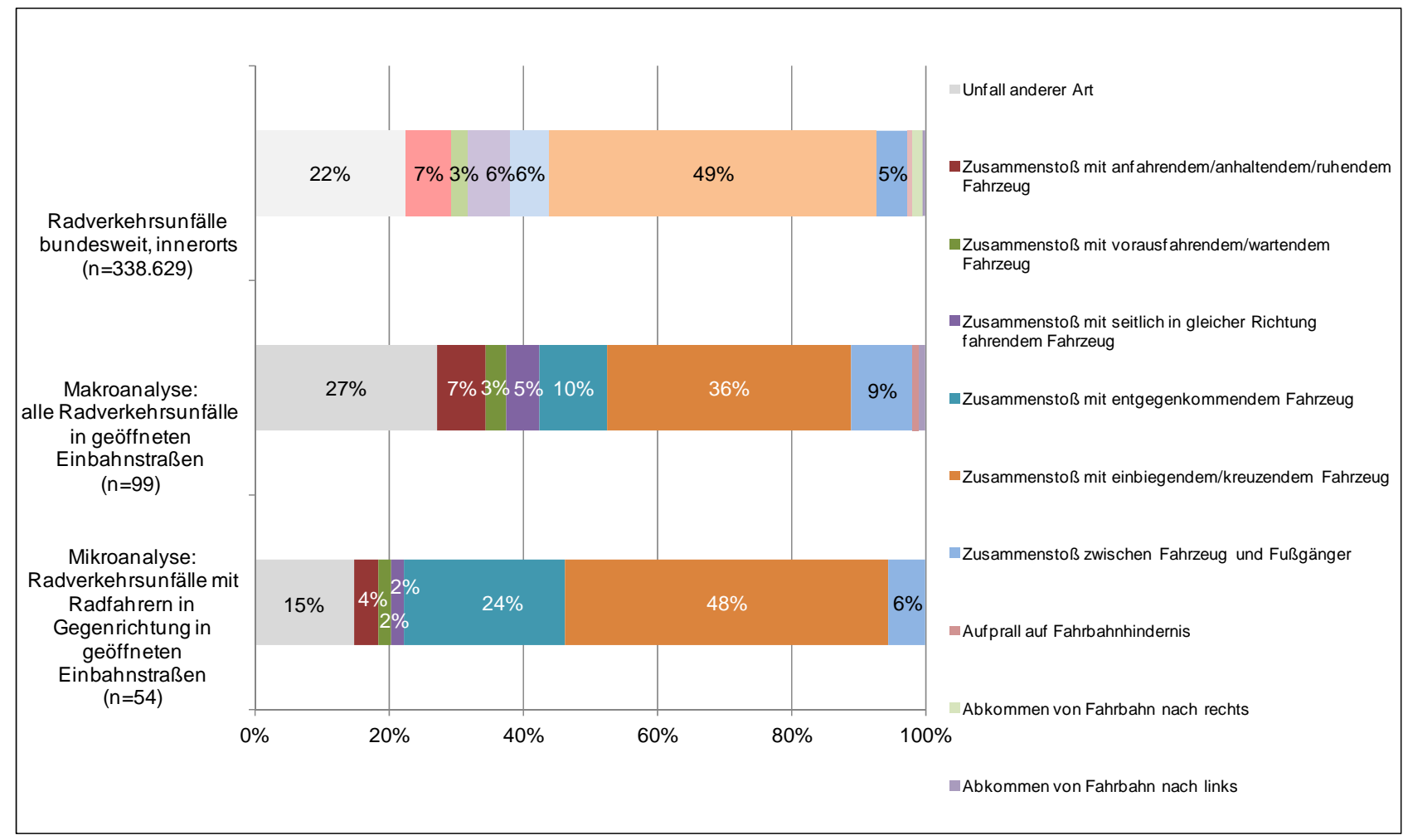

Abbildung 86: Vergleich der Unfallart in geöffneten Einbahnstraßen mit den bundesweiten Innerortsunfällen $(U(P)$, 2008-2012) ${ }^{45}$

Auffällig ist außerdem die geringere Bedeutung der Unfallart Zusammenstoß mit einbiegendem/kreuzendem Fahrzeug gegenüber den bundesweiten innerörtlichen Radverkehrsunfällen.

45 Quelle Unfälle bundesweit, innerorts: Statistisches Bundesamt (2009-2013): Fachserie 8, Reihe 7, 20082012 und Statistisches Bundesamt, Wiesbaden 2014 


\subsubsection{Unfallbeteiligte}

\section{Auswertungen zum Hauptverursacher}

Unfälle mit entgegenfahrenden Radfahrern in geöffneten Einbahnstraßen weisen in der makroskopischen Analyse anteilsmäßig häufiger Pkw (51 \%) als Radfahrer (44\%) als Hauptverursacher auf. Damit sind die Pkw in geöffneten Einbahnstraßen häufiger die Hauptverursacher von Unfällen als bei allen innerörtlichen Radverkehrsunfällen. Der Anteil der Radfahrer als Hauptverursacher ist dagegen in etwa gleich hoch.

Bezogen auf die relevanten Radverkehrsunfälle in geöffneten Einbahnstraßen in der mikroskopischen Untersuchung beträgt der Anteil der Kfz als Hauptverursacher zwei Drittel, während der Anteil der Radfahrer unter einem Drittel liegt (siehe Abbildung 87).

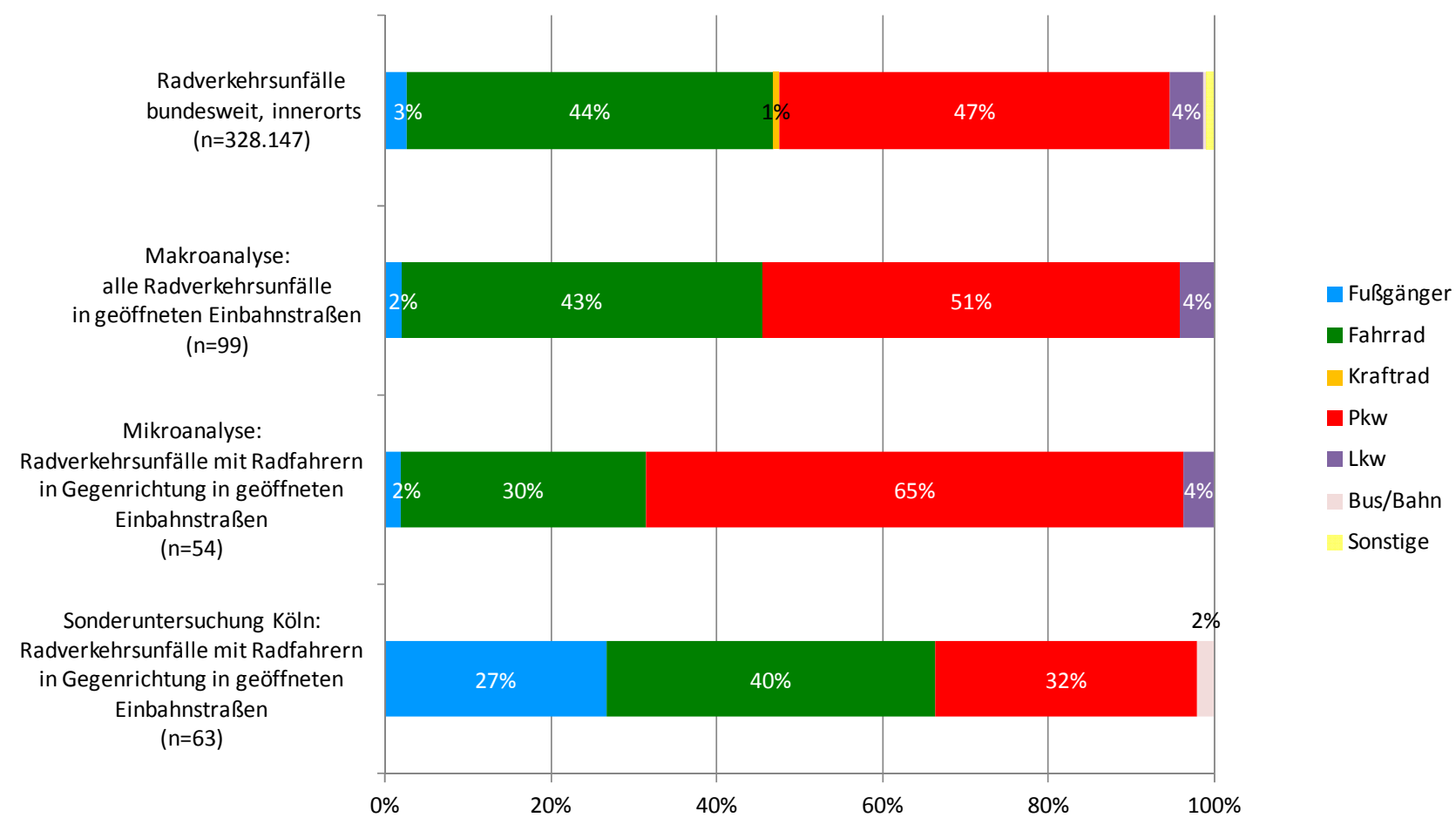

Abbildung 87: Vergleich der ersten Unfallbeteiligten (Hauptverursacher) in geöffneten Einbahnstraßen mit denen bei bundesweiten Innerortsunfällen (U(P), 2008-2012)46

In der Sonderuntersuchung zu den geöffneten Einbahnstraßen der Kölner Innenstadt sind sowohl Radfahrer als auch Pkw und Fußgänger häufige Hauptverursacher. Radfahrer haben dabei einen höheren Anteilswert als Pkw-Fahrer.

Abbildung 88 weist die Hauptverursacher für Unfälle an Zwischenknotenpunkten und auf der Strecke aus.

46 Quelle Unfälle bundesweit, innerorts: Statistisches Bundesamt (2009-2013): Fachserie 8, Reihe 7, 20082012 und Statistisches Bundesamt, Wiesbaden 2014 


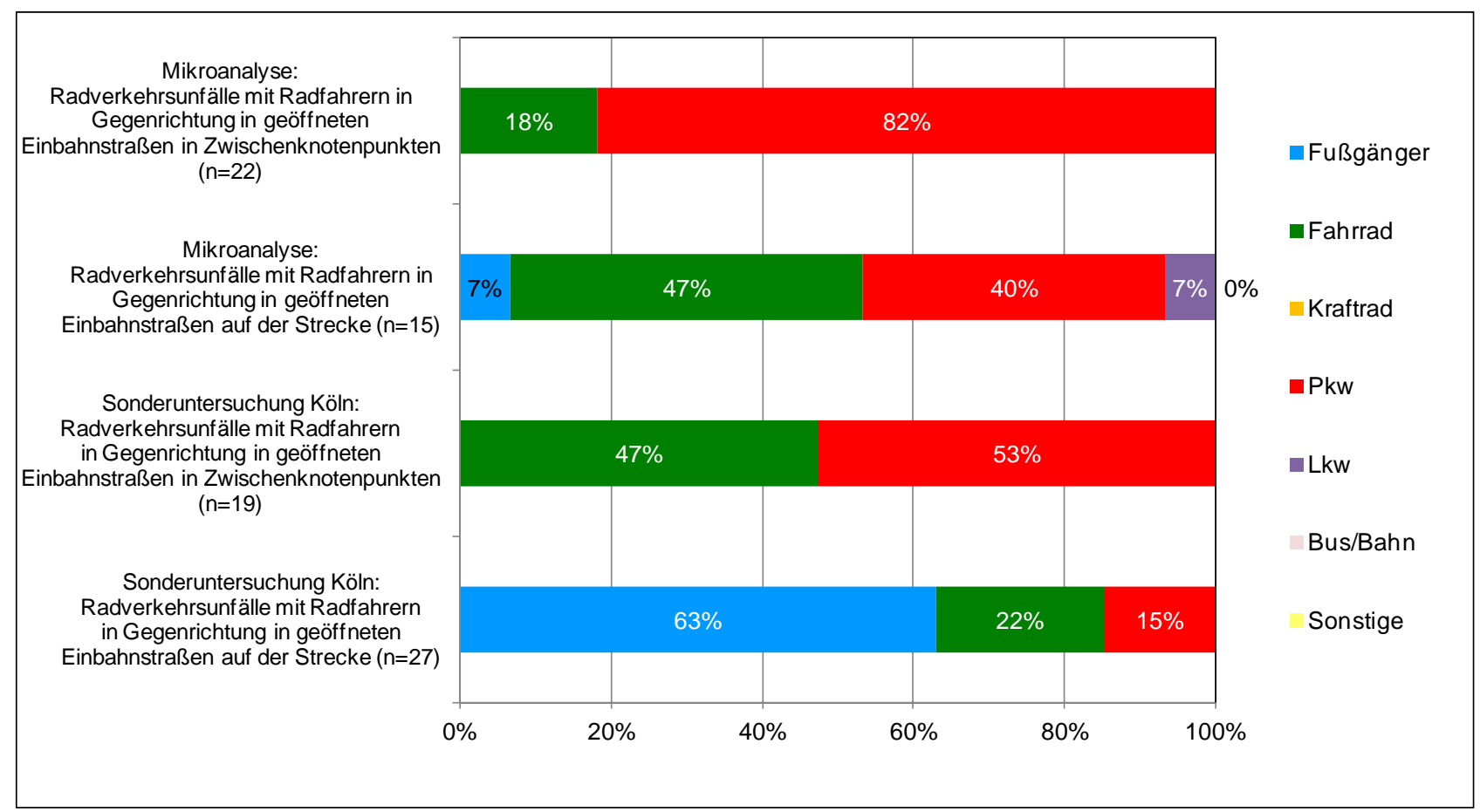

Abbildung 88: Vergleich der ersten Unfallbeteiligten (Hauptverursacher) für relevante Unfälle in geöffneten Einbahnstraßen an Zwischenknotenpunkten und auf der Strecke

In den bundesweit untersuchten 17 geöffneten Einbahnstraßen sind Pkw die weitaus häufigsten Hauptverursacher an Zwischenknotenpunkten, Radfahrer haben als Hauptunfallverursacher einen etwas höheren Anteil als Pkw auf der Strecke.

In der Sonderuntersuchung der geöffneten Einbahnstraßen in der Kölner Innenstadt sind Radfahrer und Pkw an Zwischenknoten anteilsmäßig ähnlich häufig die Hauptverursacher, auf der Strecke dagegen sind es mit Abstand die Fußgänger.

\section{Auswertung zu den Unfallgegnern}

Pkw sind in geöffneten Einbahnstraßen die häufigsten Hauptverursacher, sogar etwas häufiger als im bundesweiten innerörtlichen Durchschnitt (siehe auch Abbildung 87). Die Unfallgegner der Hauptverursacher Kfz sind am häufigsten Radfahrer, danach andere Pkw und Fußgänger. Radfahrer sind seltener die Hauptverursacher von Unfällen, ihre häufigsten Unfallgegner sind zuerst Pkw, dann andere Radfahrer. Auch Alleinunfälle sind anteilsmäßig häufiger vertreten. Alle anderen Hauptverursacher und Unfallgegner sind nicht bedeutend (siehe Abbildung 89). 


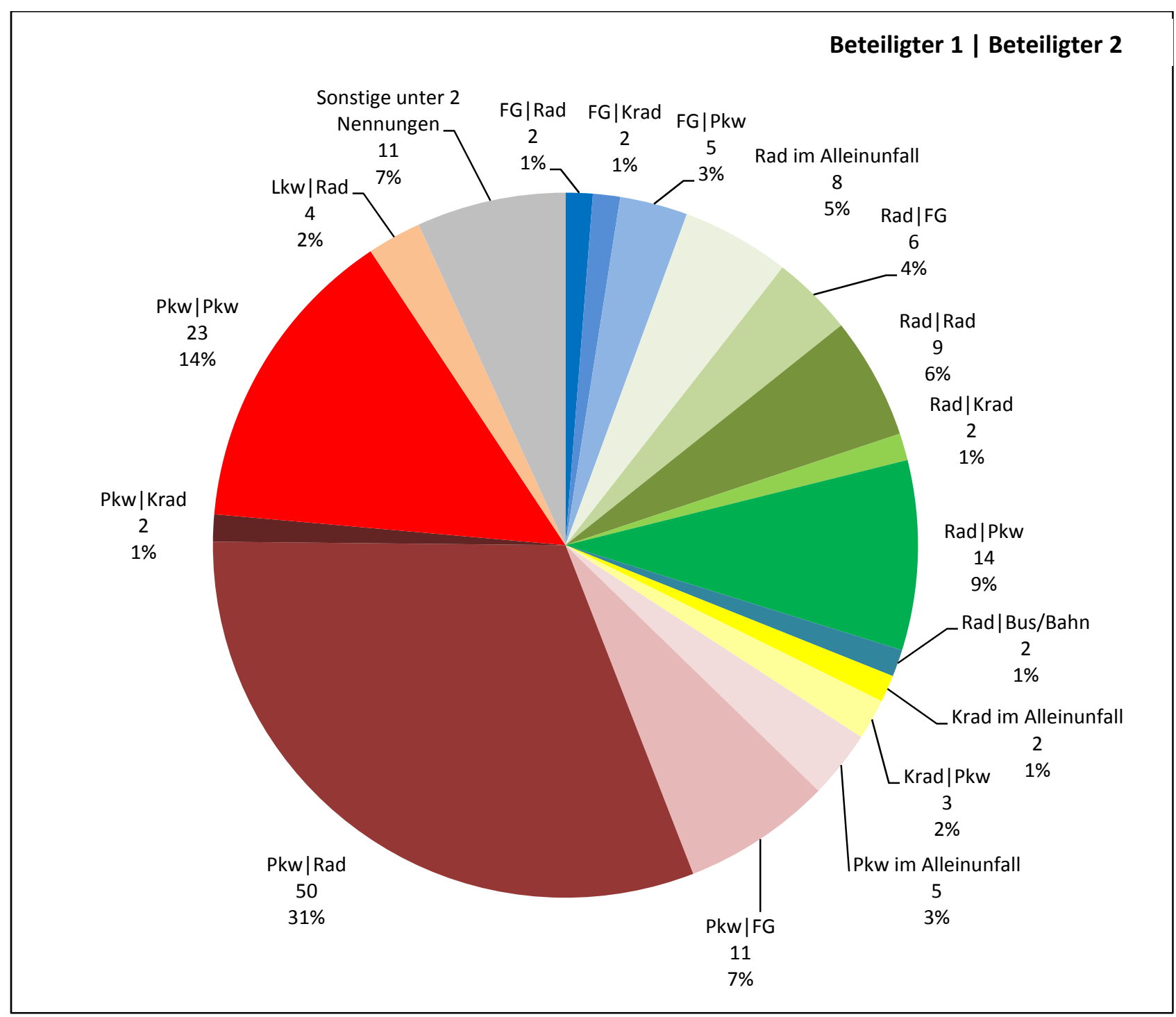

Abbildung 89: Unfallbeteiligte 1 und 2 bei allen Unfällen in geöffneten Einbahnstraßen (U(P), 2008-2012 nur Beteiligte 1 und $2, n=161$ )

Werden die Unfallgegner bei den Unfällen mit Radverkehrsbeteiligung zusammen gefasst, so ergibt sich folgendes Bild (siehe Abbildung 90). 


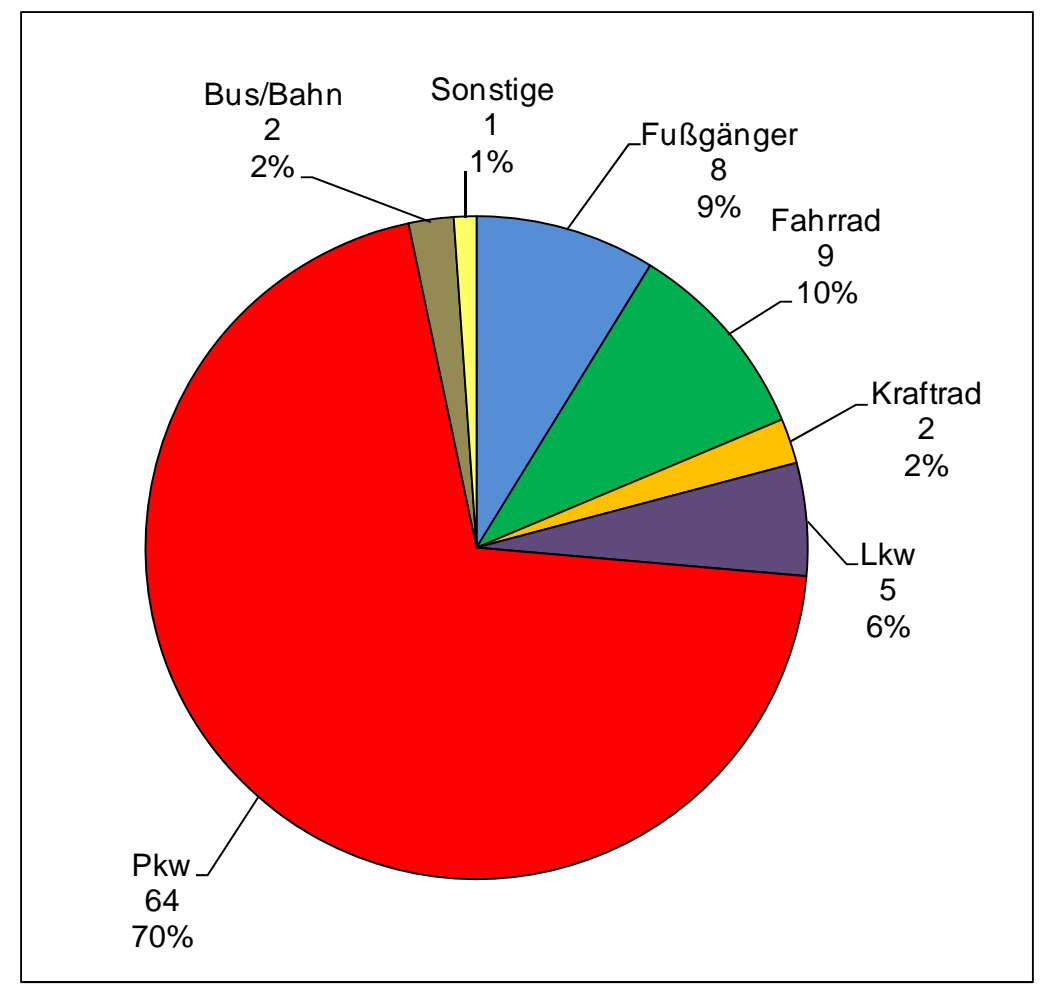

Abbildung 90: Unfallgegner bei Unfällen mit Radverkehrsbeteiligung in geöffneten Einbahnstraßen (U(P), 2008-2012, $\mathrm{n}=91$; ohne Alleinunfälle Rad)

Zwei Drittel der Unfallgegner bei Unfällen mit Radfahrerbeteiligung sind Pkw. Zu nahezu fast gleichen Anteilen sind an zweiter Stelle die Unfallgegner anderer Radfahrer oder Fußgänger zu nennen, oder es war ein Alleinunfall Fahrrad. Zu erwähnen sind auch Unfälle mit Lkw: In fünf Jahren wurden hierzu auf den untersuchten Straßen fünf Unfälle polizeilich registriert. Alle anderen Unfallgegner sind unbedeutend.

Werden bei den Unfällen mit Radverkehrsbeteiligung die Unfallgegner detailliert aufgeführt, so ergibt sich folgendes Bild (siehe Abbildung 91). 


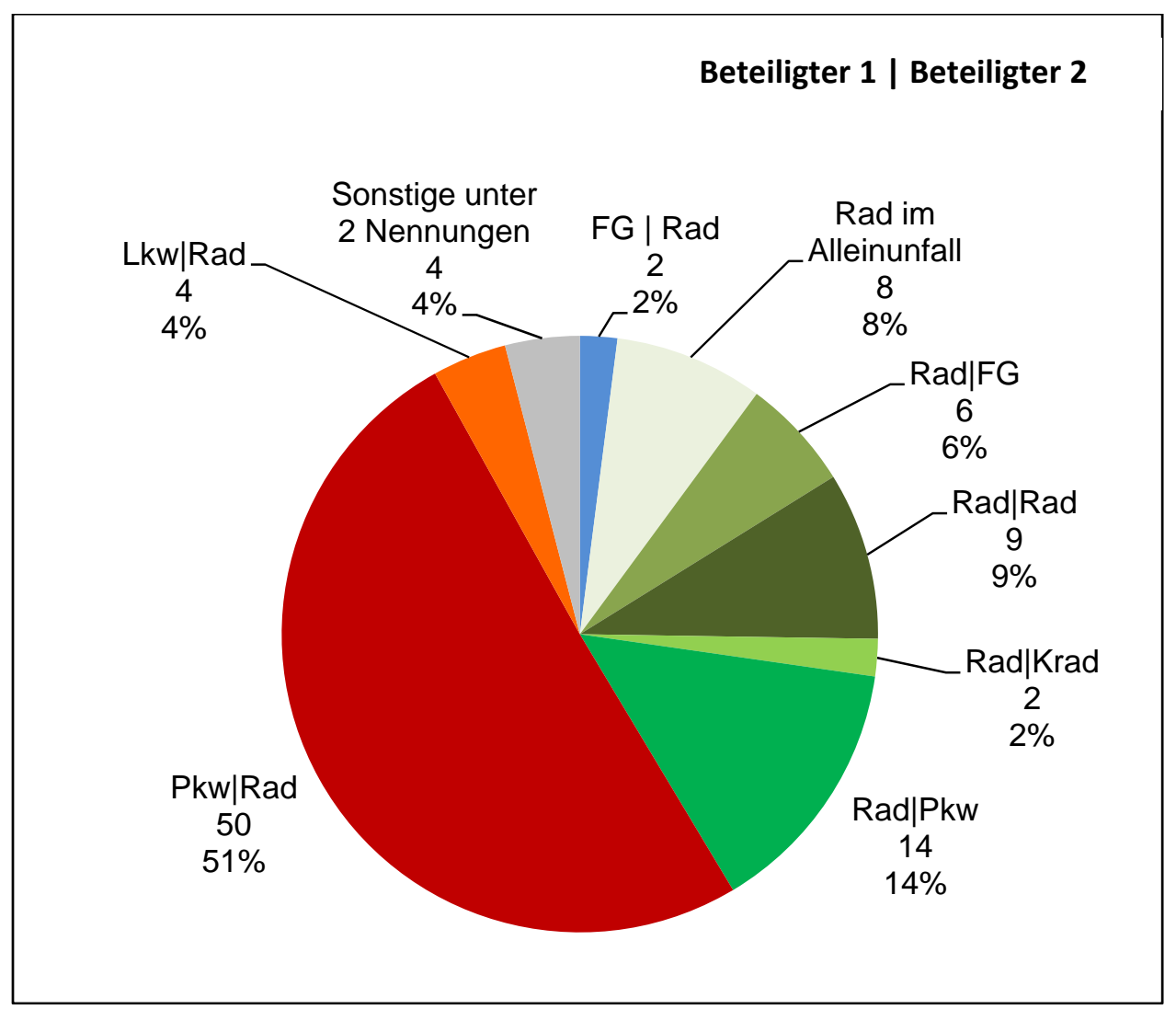

Abbildung 91: Erste Unfallbeteiligte (Hauptverursacher) bei Unfällen mit Radverkehrsbeteiligung in geöffneten Einbahnstraßen $(U(P), 2008-2012$, nur Beteiligte 1 und 2, $n=99)$

Die Hauptverursacher an allen Unfällen mit Radverkehrsbeteiligung in geöffneten Einbahnstraßen sind zur Hälfte Pkw und zu etwas mehr als einem Drittel Radfahrer.

Werden nur die Unfälle mit den Beteiligten Pkw und Rad betrachtet, sind die Radfahrer nur bei einem Fünftel der Unfälle die Hauptverursacher (siehe Abbildung 92). Dies sind noch weniger als in Fahrradstraßen (dort ein Viertel). 


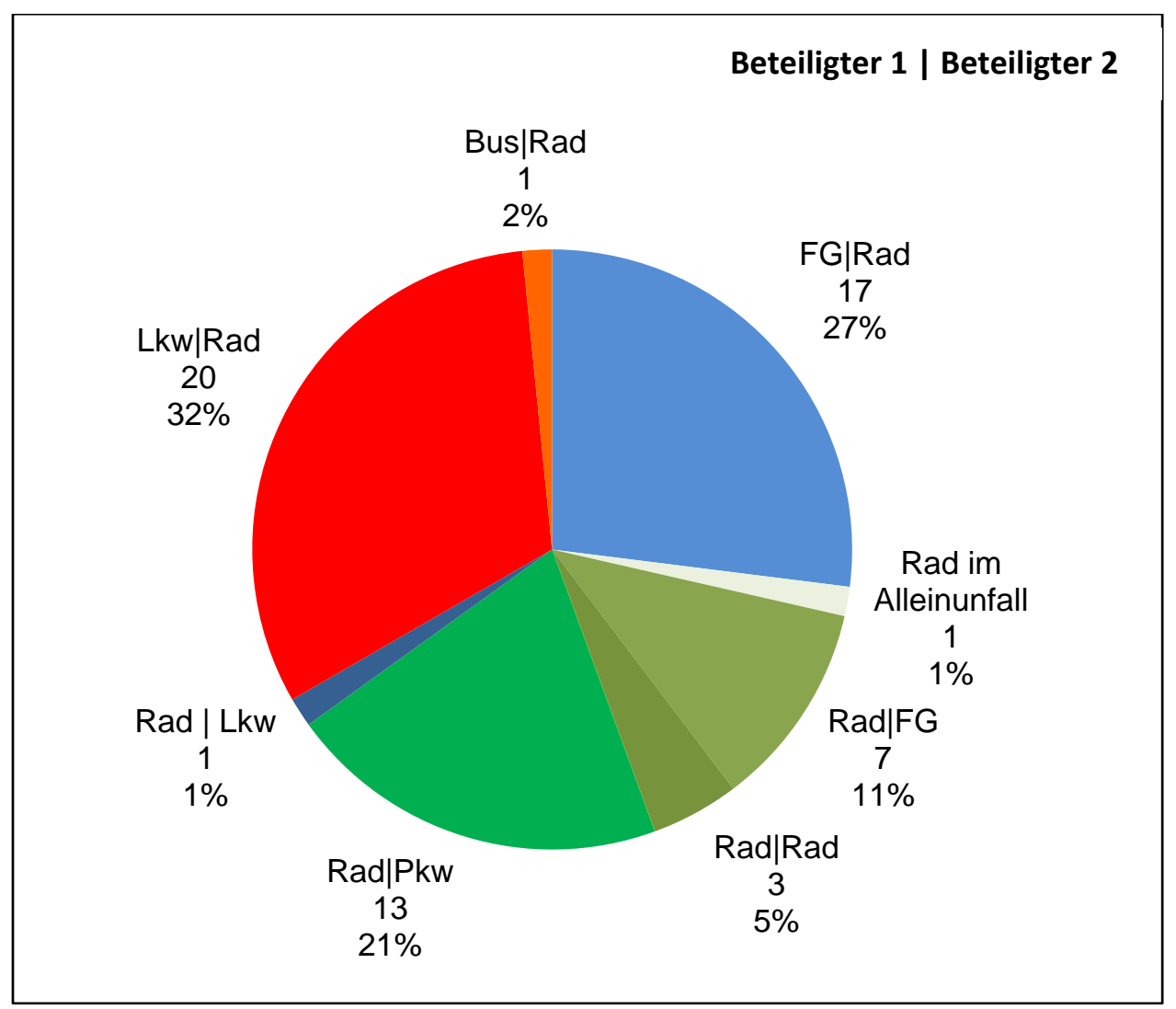

Abbildung 92: Erste Unfallbeteiligte (Hauptverursacher) bei Unfällen der Sonderuntersuchung geöffnete Einbahnstraßen in der Kölner Innenstadt (U(P), 2008-2012, nur Beteiligte 1 und 2, n=63)

\section{Auswertungen zu den Unfallbeteiligten als Haupt-IAlleinverursacher und Mitverursacher}

Unfälle, für die nur ein Unfallbeteiligter genannt wird, gelten als Alleinunfälle. Der erste Unfallbeteiligte wird als Hauptverursacher gewertet. Bei Unfällen mit mehreren Unfallbeteiligten sind die weiteren Unfallbeteiligten (hier die Unfallbeteiligten Zwei bis Vier) Mitverursacher, wenn auch innen Unfallursachen zugeordnet wurden. Wurden den weiteren Unfallbeteiligten keine Unfallursachen zugeordnet, gelten diese nicht als Mitverursacher, sondern als Unfallbeteiligte ohne Ursache.

Die Unfallbeteiligten bei Radverkehrsunfällen in geöffneten Einbahnstraßen in diesen Kategorien zeigt die Abbildung 93. 


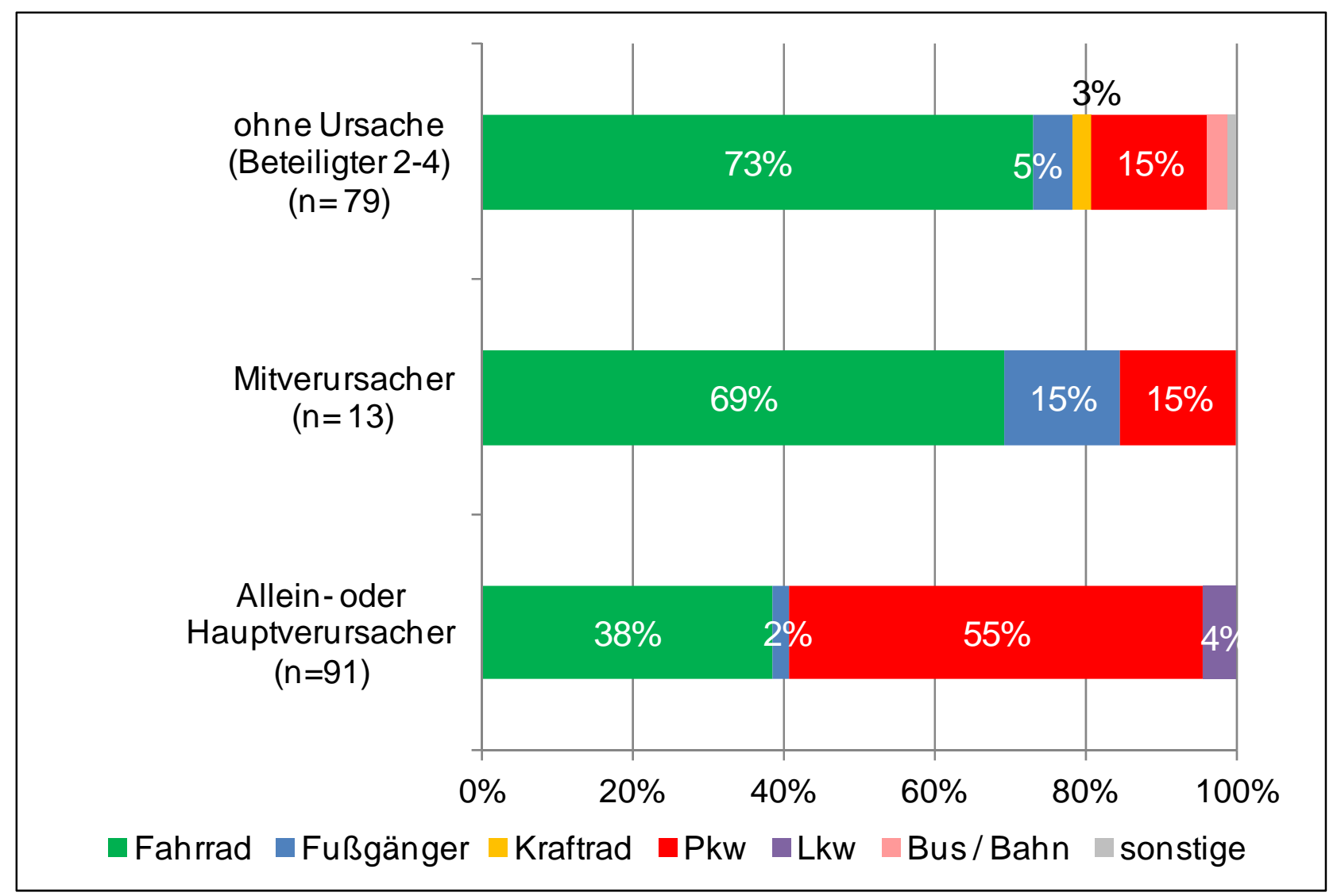

Abbildung 93: Unfallverursacher bei Unfällen mit Radverkehrsbeteiligung in geöffneten Einbahnstraßen (U(P), 20082012, ohne Alleinunfälle Fahrrad: $n=99$ )

Der Pkw überwiegt als Allein- oder Hauptverursacher von Radverkehrsunfällen ${ }^{47}$. Bei den Unfällen mit Lkw-Beteiligung waren diese stets die Hauptverursacher.

Radfahrer werden zu zwei Drittel als Mitverursacher aufgeführt. Fast vier Fünftel der Beteiligten ohne Ursache waren Radfahrer.

47 Ohne Alleinunfälle Fahrrad 


\subsubsection{Unfallursachen}

In der makroskopischen Unfallanalyse wurden die Unfallursachen detailliert untersucht und nach Unfallbeteiligung ausgewertet. Abbildung 94 zeigt die Unfallursachen 1 bis 3 der Radfahrer für alle Unfälle mit Radverkehrsbeteiligung in geöffneten Einbahnstraßen.

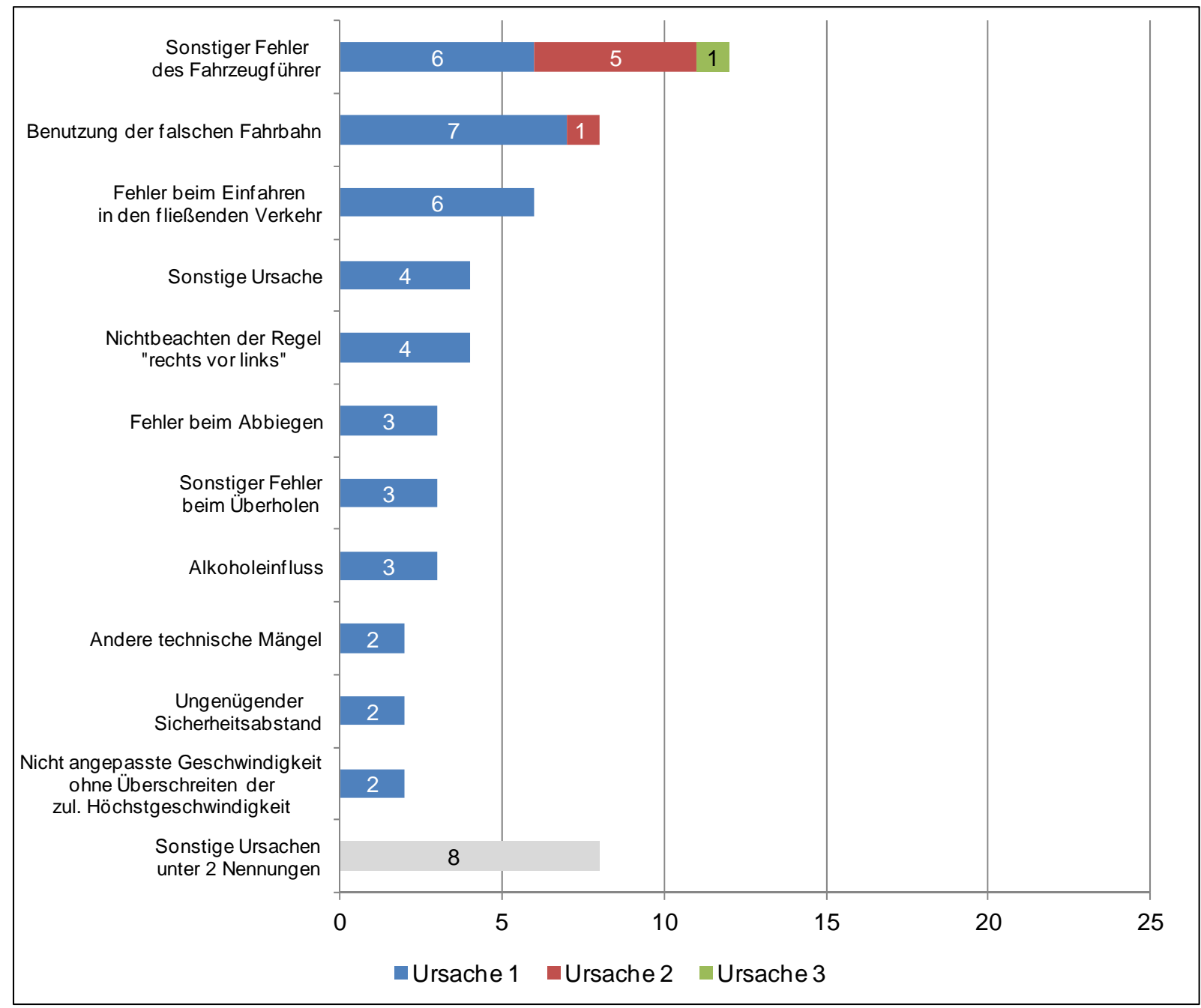

Abbildung 94: Unfallursachen aller beteiligten Radfahrer bei Unfällen mit Radverkehrsbeteiligung in geöffneten Einbahnstraßen in der Makroanalyse (U(P), 2008-2012, n=57, Mehrfachnennungen möglich)

Als häufigste Ursache sind für Radfahrer sonstige Fehler angegeben worden. An zweiter Stelle lieg die Benutzung der falschen Fahrbahn: Offensichtlich fahren auch in geöffneten Einbahnstraßen Radfahrer mitunter illegal auf dem Gehweg. 
Abbildung 95 zeigt die Unfallursachen 1 bis 3 für alle übrigen Beteiligten außer Radfahrer an allen Unfällen, an denen Radfahrer beteiligt sind.

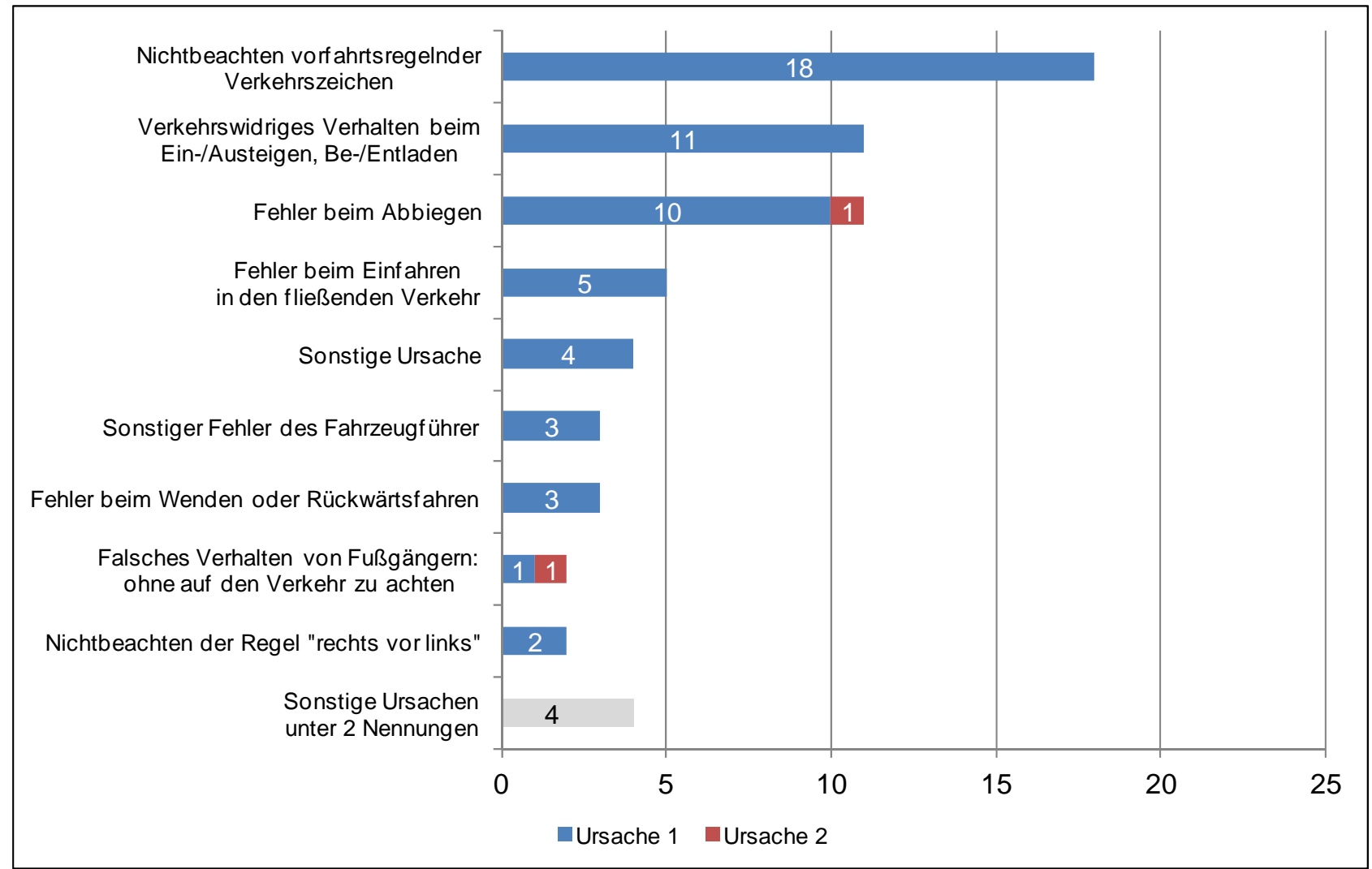

Abbildung 95: Unfallursachen aller beteiligten Unfallgegner außer Radfahrer an Unfällen mit Radverkehrsbeteiligung in geöffneten Einbahnstraßen in der Makroanalyse $(U(P), 2008-2012, n=63$, Mehrfachnennungen möglich)

Bei den Unfallverursachern, die nicht Radfahrer waren, dominierten Vorfahrtsvergehen. Daneben werden auch in den geöffneten Einbahnstraßen Sicherheitsdefizite durch das Parken offensichtlich.

In der mikroskopischen Analysephase wurden die Unfallursachen nach Hauptverursacher Pkw und Fahrrad sowie nach Unfallort „Strecke“ für die Unfälle in geöffneten Einbahnstraßen der bundesweiten Untersuchung ausgewertet. Die Fallzahlen sind so gering, dass sich eine Aussage kaum noch ableiten lässt. Bei den Radfahrern scheint öfter das Überholen Unfallursache zu sein.

In der Sonderuntersuchung zu den geöffneten Einbahnstraßen in der Kölner Innenstadt wurden ebenfalls die Unfallursachen nach Hauptverursacher Pkw und Fahrrad sowie nach Unfallort „Strecke" für die Unfälle in geöffneten Einbahnstraßen ausgewertet. Auch hier sind die Fallzahlen so gering, dass sich eine Aussage kaum noch ableiten lässt. Deutlich werden in der Kölner Innenstadt die Unfallursachen der Fußgänger: Falsches Verhalten von Fußgängern durch plötzliches Hervortreten und ohne auf den Verkehr zu achten.

In der mikroskopischen Analyse und für die Sonderuntersuchung der geöffneten Einbahnstraßen in der Kölner Innenstadt wurden zudem die Unfallursachen an den Zwischenknotenpunkten nach Hauptverursacher ausgewertet: Es dominieren die Vorfahrtverstöße vor allem bei den Pkw. 


\subsubsection{Zwischenfazit Unfalluntersuchung in geöffneten Einbahnstraßen}

Die Verkehrssicherheit in für den Fahrradverkehr in Gegenrichtung geöffnete Einbahnstraßen wurde im Rahmen der durchgeführten kommunalen Befragung von der überwiegenden Mehrheit der Kommunen als nicht problematisch bewertet: Bundesweit wurden nur wenige geöffnete Einbahnstraßen mit Unfällen genannt. Aus den genannten geöffneten Einbahnstraßen geschahen nur in 17 Einbahnstraßen Radverkehrsunfälle in Gegenrichtung. Als Vergleichskollektiv zu der möglicherweise subjektiven Einschätzung der in der Kommunalbefragung befragten Akteure wurden alle Unfälle mit Radverkehrsbeteiligung in allen geöffneten Einbahnstraßen der Kölner Innenstadt im selben Untersuchungszeitraum analysiert.

Nur etwa jeder dritte Radverkehrsunfall (54 Unfälle) in als problematisch genannten geöffneten Einbahnstraßen war ein Unfall mit Radverkehr in Gegenrichtung (siehe Kapitel 2.3.2.). In der Kölner Innenstadt waren sogar nur etwas mehr als ein Fünftel der Unfälle relevante Radverkehrsunfälle. Für die Kölner Innenstadt heißt das konkret, dass in den 200 geöffneten Einbahnstraßen pro Jahr nur insgesamt ca. 13 Unfälle mit Radfahrern in Gegenrichtung polizeilich registriert wurden. Somit hat die Sonderuntersuchung in der Kölner Innenstadt ergeben, dass nur wenige geöffnete Einbahnstraßen überhaupt relevante Unfälle aufweisen.

Die wenigen Unfälle, die als relevante Unfälle untersucht werden konnten, fanden überwiegend an den Knotenpunkten statt.

Bei den Streckenunfällen dominierten einige wenige Straßen, die durch eine hohe Nutzungsintensität des Seitenraums charakterisiert sind. Unfälle im Längsverkehr, z.B. im Begegnungsfall spielen nur eine sehr untergeordnete Rolle.

Die Schwere der Radverkehrsunfälle in geöffneten Einbahnstraßen ist mit $9 \%$ Schwerverletzten deutlich geringer als im bundesweiten innerörtlichen Durchschnitt (17\% Schwerverletzte). Betrachtet man nur den Anteil der Unfälle, die sich mit Radfahrern ereignen, die in der Einbahnstraße in Gegenrichtung unterwegs sind, ist dieser noch einmal geringer (4 \% bzw. 6 \% Schwerverletzte).

Auch in für den Radverkehr geöffneten Einbahnstraßen überwiegen die Einbiegen/Kreuzen-Unfälle bei den relevanten Unfällen. Diese machen drei Viertel aller Unfälle an Knotenpunkten aus und verdeutlichen die dominierende Konfliktsituation zwischen einbiegendem $\mathrm{Kfz}$ und dem in der geöffneten Einbahnstraße fahrenden Radfahrer.

Eine Auffälligkeit der Untersuchung in der Kölner Innenstadt war der hohe Anteil von Überschreiten-Unfällen (30\%). Die mikroskopische Unfalluntersuchung konnte zeigen, dass diese Überschreiten-Unfälle in geöffneten Einbahnstraßen vor allem in den Haupteinkaufsstraßen der Kölner Innenstadt mit hoher Fußgängerfrequenz passieren. Hier achten Fußgänger beim Überqueren der Straße oft nicht auf den aus entgegen der allgemeinen Fahrtrichtung kommenden Fahrradverkehr.

Der Pkw ist in den bundesweit untersuchten geöffneten Einbahnstraßen bei den insgesamt wenigen Unfällen der häufigste Unfallgegner und der häufigste Hauptverursacher. Bei Unfällen zwischen Pkw und Radfahrern am Zwischenknotenpunkt ist der Pkw zu vier Fünfteln der Hauptverursacher. Bei den bereits erwähnten Überschreiten-Unfällen sind überwiegend die Fußgänger Hauptverursacher.

Insgesamt erweist sich die geöffnete Einbahnstraße als ein grundsätzlich sehr sicheres Infrastrukturelement. 


\subsection{Ergebnisse der Verhaltensbeobachtung}

Die Verhaltensbeobachtung in den geöffneten Einbahnstraßen umfasste beispielhaft fünf Standorte, von denen drei gleichzeitig auch als Fahrradstraßen ausgewiesen sind. Die Auswahl der Standorte erfolgte aufgrund der unterschiedlichen Knotenpunktcharakteristik in Kombination mit einer unterschiedlichen Anzahl relevanter Unfälle. Die Kfz-Belastung der Knotenpunkte variierte ebenfalls von hoch $(11.200 \mathrm{Kfz} / 24 \mathrm{~h})$ bis gering $(600 \mathrm{Kfz} / 24 \mathrm{~h})$. Die Standorte wiesen auch unterschiedliche Radverkehrsbelastungen auf, diese reichten von 81 Radfahrern/24h bis zu 1.320 Radfahrer/24h.

Bei den Kombinationen der Rad- und Kfz-Verkehrsstärke zeigt sich eine deutliche Diskrepanz zwischen den alleinigen Einbahnstraßen, die für Radfahrer geöffnet sind und den geöffneten Einbahnstraßen, welche gleichzeitig auch als Fahrradstraßen ausgewiesen sind. Während an den Standorten mit Straßen, die gleichzeitig auch Fahrradstraßen sind, der Radverkehrsanteil überwiegt, liegt bei den reinen geöffneten Einbahnstraßen trotz ihrer sehr zentralen Lage der KfzAnteil bei $88 \%$, bzw. $90 \%$.

In der Auswertung zeigt sich wie bereits bei den Fahrradstraßen, dass ein Zusammenhang zwischen Konflikthäufigkeit und Verkehrsstärken nicht belegt werden konnte. So weist der am stärksten durch den KfZ- Verkehr frequentierte Knotenpunkt äußerst geringe Konfliktwerte auf. Auch am Standort mit dem höchsten Radverkehrsanteil wurden keine Konflikte beobachtet.

\section{Interaktionen}

Auf den fünf beobachteten Einbahnstraßen (4 Knotenpunkte und eine Strecke) wurden 475 Interaktionen mit zwölf Konflikten beobachtet, was einer Konfliktrate von 2,5 \% entspricht. In 8 Fällen fuhr der Radfahrer entgegen und in 4 Fällen mit der Fahrtrichtung des PKW. Die Konflikte mit Radfahrern entgegen der Einbahnrichtung entstanden hauptsächlich aufgrund von Vorfahrtsmissachtungen ( 7 Konflikte) sowie durch eine falsche Fahrbahnbenutzung (Kfz biegt in weitem Radius ab und gelangt auf die Fahrbahn des entgegenkommenden Radfahrers). 


\section{Beispielhafte Situationen:}

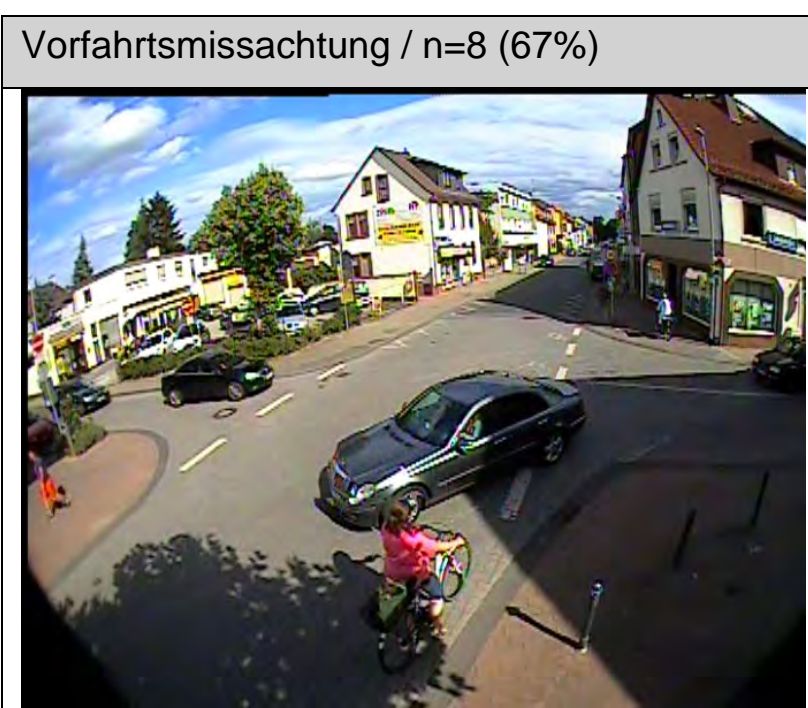

Abbildung 96: Wartepflichtiger Kfz nimmt beim Linkseinbiegen einem Radfahrer in Gegenrichtung der Einbahnstraße die Vorfahrt, dieser bremst deutlich ab.

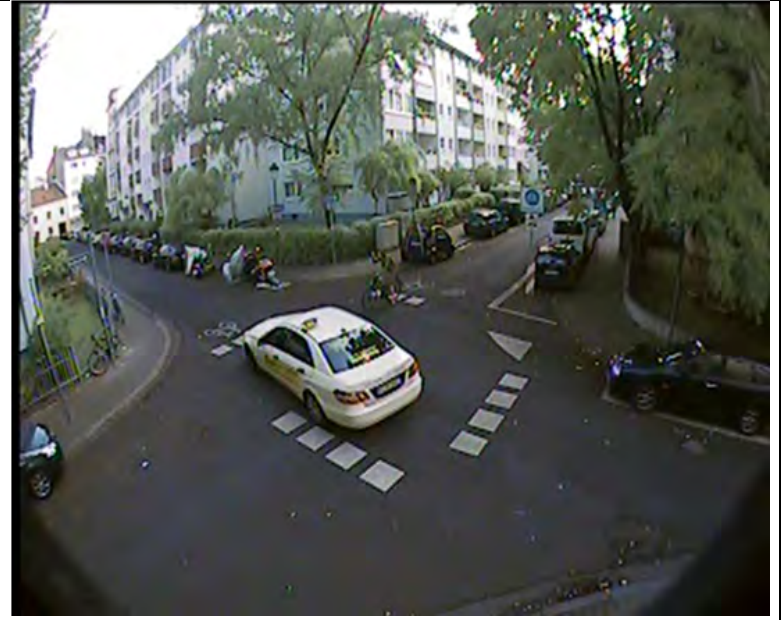

Abbildung 97: Wartepflichtiger Kfz nimmt einem aus Gegenrichtung der Einbahnstraße kommenden Radfahrer die Vorfahrt, dieser muss stark bremsen.

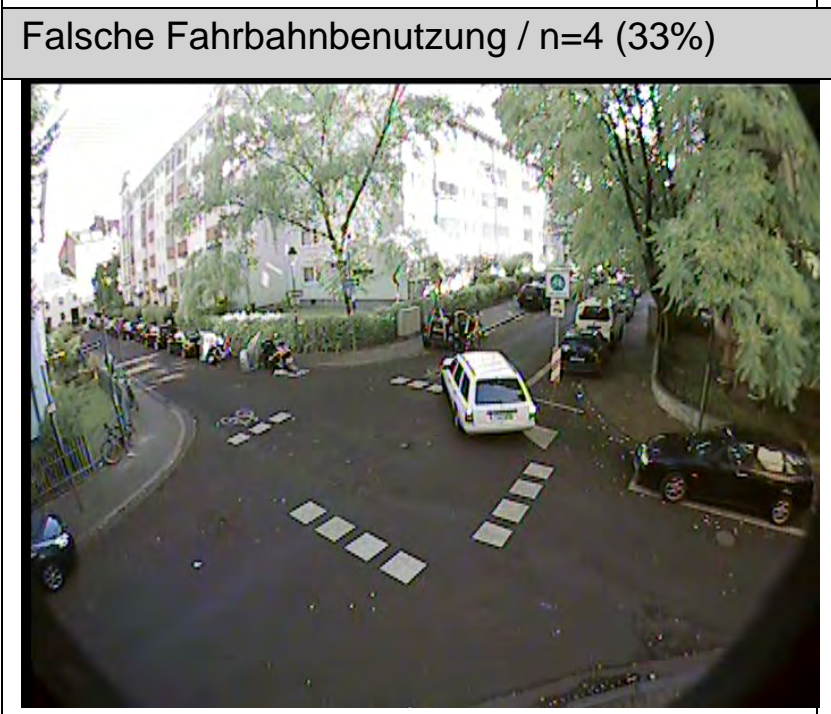

Abbildung 98: Kfz biegt in großem Radius in eine Straße ab, entgegenkommender Radfahrer in Gegenrichtung der Eibahnstraße weicht aus.

\section{Untersuchte Standorte}

Standort 1: Unter den untersuchten geöffneten Einbahnstraßen befindet sich nur ein Streckenabschnitt. Dieser wurde als einziger Streckenabschnitt in der Kommunalbefragung als problematisch gemeldet und wies zugleich in den letzten fünf Jahren vier relevante Unfälle mit Radfahrern gegen die Einbahnrichtung auf. Aufgrund der schmalen Fahrgasse (2,40 m Breite) kamen die Radfahrer bei Kfz-Gegenverkehr zum Teil mit Pollern in Konflikt und verunfallten. Bei der Videobeobachtung wurden keine Konflikte festgestellt, allerdings waren die Poller zur Zeit der Videobeobachtung aufgrund von Bauarbeiten entfernt. Die ermittelte Geschwindigkeit der Kfz liegt mit einer $V_{85}$ von $33,5 \mathrm{~km} / \mathrm{h}$ leicht über der zulässigen Höchstgeschwindigkeit von $30 \mathrm{~km} / \mathrm{h}$. 


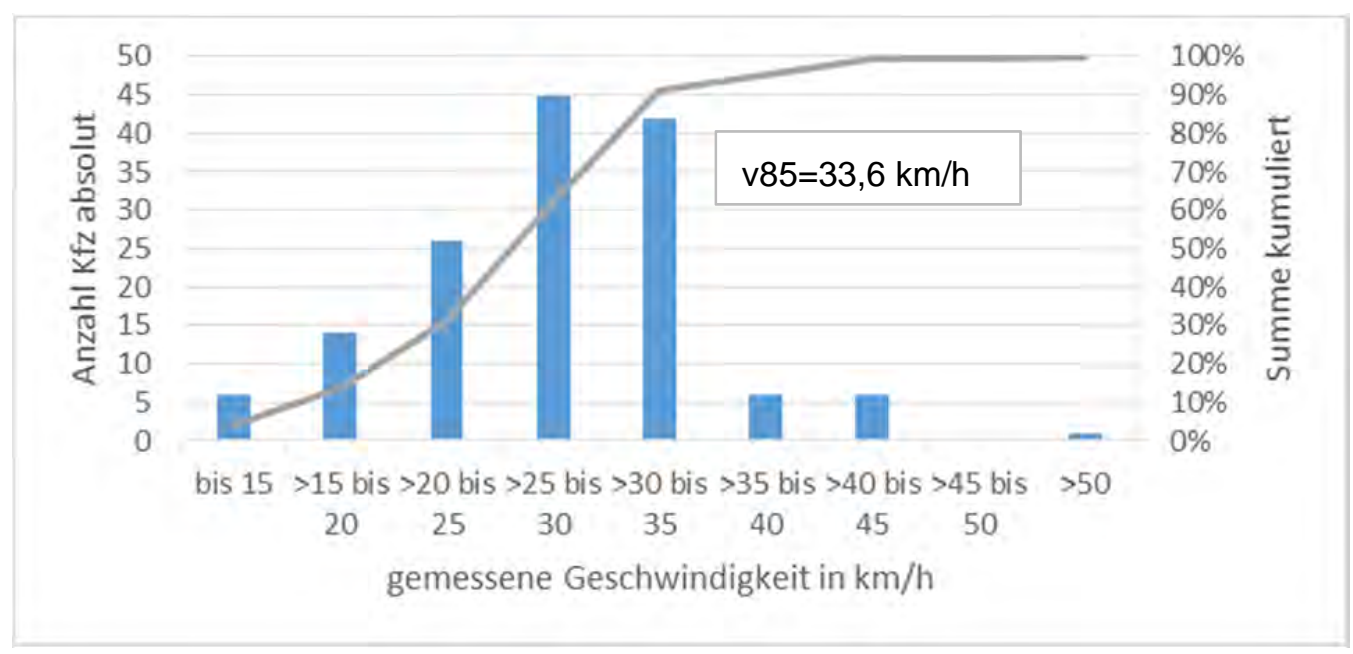

Abbildung 99: $\quad$ Gemessene Geschwindigkeit, Standort $1(n=146)$

Standort 2: Hier begegnen sich zwei geöffnete Einbahnstraßen, die Vorfahrt ist Rechts-vor-links geregelt. Am Knotenpunkt geschahen zwei relevante Unfälle in fünf Jahren. In der Videobeobachtung wurden 42 Interaktionen, aber kein Konflikt beobachtet. Der Knotenpunkt weist als Besonderheit einen Radweg in Gegenrichtung an zwei Zufahrten auf, die Bebauung reicht bis nahe an die Fahrbahn. Trotz der dadurch eingeschränkten Sichtbeziehung und der „doppelten“ Einbahnregelung weist der Knotenpunkt nur eine geringe Unfallbelastung und keine Konflikte auf.

Standort 3: Dieser Standort wies eine Belastung von mehr als $10.000 \mathrm{Kfz} . / 24 \mathrm{~h}$ auf. Zwei Einbahnstraßen führen auf den Knoten zu, eine Einbahnstraße führt vom Knotenpunkt weg. Eine zuführende Einbahnstraße ist wartepflichtig. In fünf Jahren haben sich hier 4 relevante Unfälle ereignet. Von 234 Interaktionen zwischen $\mathrm{Kfz}$ und Radfahrern ereigneten sich 2 leichte Konflikte (0,9\%), davon ein Konflikt mit einem Radfahrer entgegen der Einbahnrichtung.

Standort 4 weist drei zuführende und eine wegführende Einbahnstraße auf, diese sind Rechts-vorlinks geregelt. Der Knotenpunkt liegt in einem Wohngebiet und ist gut einsehbar, es gibt keine Markierungen mit Bezug auf die Einbahnstraße auf der Fahrbahn. In fünf Jahren wurden keine relevanten Unfälle verzeichnet. Während der Videobeobachtung konnten an diesem Standort allerdings sechs Konflikte beobachtet werden, in denen die Vorfahrt missachtet wurde, davon in vier Fällen durch Radfahrer, die entgegen der Einbahnstraße fuhren. Die 6 beobachteten Konflikte machen rund 5\% der Interaktionen aus.

Standort 5 liegt ebenfalls in einem Wohngebiet, beide Straßen sind geöffnete Einbahnstraßen. Alle vier Zufahrten sind mit Wartelinien versehen, die beiden Zufahrten, aus denen nur Radverkehr kommt, zusätzlich mit Fahrradpiktogrammen. Die Vorfahrt ist durch Rechts-vor-links geregelt. Die Sichtbeziehungen sind eingeschränkt durch Hecken, aber auch durch im Kreuzungsbereich parkende Pkw. In fünf Jahren haben sich 3 relevante Unfälle ereignet. Die Videobeobachtung hat eine Konfliktrate von 7 \% (4 Konflikte - davon 3 mit Radfahrern entgegen der Einbahnrichtung-bei 57 Interaktionen) ergeben. Dies waren in zwei Fällen Missachtung der Vorfahrt durch Kfz und in weiteren zwei Fällen eine falsche Fahrbahn-Benutzung.

\subsubsection{Zwischenfazit der Verhaltensbeobachtung}

Die Videobeobachtung in geöffneten Einbahnstraßen umfasste beispielhaft fünf Standorte: einen Streckenabschnitt sowie vier Knotenpunkte. Die beobachteten Standorte wiesen mit bis zu 4 relevanten Unfällen in fünf Jahren ein sehr geringes Unfallgeschehen auf. Die Konflikthäufigkeit war mit 2,5\% in allen geöffneten Einbahnstraßen sehr gering bei einer Spannweite von 0 bis $7 \%$. Alle Standorte weisen unterschiedliche Charakteristika auf. Es lassen sich keine Zusammenhänge 
zwischen Konflikten und Infrastruktur nachweisen. Es ergaben sich lediglich Hinweise auf ein geringes Gefährdungspotential, aber keine verallgemeinerbaren Erkenntnisse. Analog zur Studie von PGVI BIS aus dem Jahr 2001 finden sich in den exemplarisch untersuchten Knotenpunkten ähnliche Verhaltensmuster, insbesondere an Knotenpunkten mit Rechts-vor-links-Regelung besteht ein erhöhtes Konfliktpotential. Hier ist insbesondere auf die Bedeutung der Einhaltung ausreichender Sichtbeziehungen hinzuweisen (vgl. Literaturkapitel 4.1).

\subsection{Resümee geöffnete Einbahnstraßen}

Geöffnete Einbahnstraßen gehören mittlerweile zum Standard der Radverkehrsplanung und werden dementsprechend in allen Ortsgrößenklassen sehr häufig eingesetzt. Alle befragten Städte über 50.000 Einwohner nutzen die Möglichkeit der Öffnung von Einbahnstraßen für den Radverkehr.

Die aktuellsten umfassenden Untersuchungen zu geöffneten Einbahnstraßen stammen aus den Jahren 1997 und 2001. Darin wird ausgeführt, dass geöffnete Einbahnstraßen im Vergleich zu nicht geöffneten Einbahnstraßen nicht unfallauffälliger sind, sondern einen positiven Einfluss auf die Verkehrssicherheit und den Verkehrsablauf haben. Wichtige Erkenntnisse waren, dass Kfz bei Begegnungen mit Radfahrern deutlich langsamer als bei unbehinderter Fahrt fahren, beim Überholen in gleicher Fahrtrichtung kommt es dagegen zu einer Beschleunigung. Auch konnte festgestellt werden, dass durch die Öffnung der Einbahnstraße Sicherheitsgewinne vor allem für Fußgänger zu verzeichnen sind, da Radfahrer nun auf der Fahrbahn fahren und nicht mehr auf dem Gehweg Fußgänger gefährden. Es gibt aber auch Hinweise, dass geöffnete Einbahnstraßen vor allem von Gelegenheitsradfahrern kritisch gesehen werden.

Geöffnete Einbahnstraßen werden von den Kommunen als sicher wahrgenommen und bewertet. Aus der Bevölkerung liegen ebenfalls nur wenige Beschwerden vor. Nur 25 von 2.373 gemeldeten Einbahnstraßen (1\%) wurden von Kommunen in der Kommunalbefragung als problematisch eingestuft.

In nur $36 \%$ der Unfälle in als problematisch genannten geöffneten Einbahnstraßen fanden Unfälle mit Radverkehr in Gegenrichtung statt. In der Kölner Innenstadt waren dies sogar nur $22 \%$ der Radverkehrsunfälle. Für die Kölner Innenstadt heißt das konkret, dass in den 200 geöffneten Einbahnstraßen pro Jahr nur insgesamt ca. 13 Unfälle mit Radfahrern in Gegenrichtung polizeilich registriert wurden.

Sowohl die Unfallschwere (4 bis $6 \%$ Schwerverletzte) als auch die Häufigkeit von Unfällen mit Radverkehr in Gegenrichtung liegt unter der des Vergleichskollektivs des innerörtlichen Straßennetzes, Diese Unfälle machten in der flächenhaften Analyse der 200 Kölner Einbahnstraßen nur $20 \%$ aller registrierten Unfälle aus.

Die meisten Unfälle finden auch in geöffneten Einbahnstraßen an Knotenpunkten statt und zählen zum Typ Einbiegen/ Kreuzen. Dieser Befund ist weitgehend Deckungsgleich mit den Befunden aus der ausgewerteten älteren Literatur. Die typische Konstellation ist das Übersehen eines kreuzenden Radfahrers, der aus der Gegenrichtung die Einbahnstraße befährt.

Unfälle im Längsverkehr wurden in der Stichprobe durch Überschreiten-Unfälle dominiert. Hier hat die vertiefende Mikroanalyse ergeben, dass auf den 5 Haupteinkaufsstraßen mit hoher Fußgängerfrequenz aus der Stichprobe fast alle dieser Überschreiten Unfälle stattfanden. Es handelt sich also um eine Sondersituation. Konflikte und Unfälle im Gegenverkehr konnten nur in wenigen Einzelfällen festgestellt werden.

Der Pkw ist in den geöffneten Einbahnstraßen bei den insgesamt wenigen Unfällen der häufigste Unfallgegner und der häufigste Hauptverursacher. Als Unfallursachen an den Knotenpunkten 
wurden den Unfallverursachern Pkw vor allem Vorfahrtverstöße zugeordnet. Fußgänger als Unfallverursacher treten nur auf den bereits erwähnten Geschäftsstraßen in Erscheinung.

Insgesamt erweist sich die geöffnete Einbahnstraße als ein Infrastrukturelement mit geringer Unfallbelastung und geringer Konfliktdichte. Die vorhandenen begrenzten Risikofaktoren wie Vorfahrtsverstöße an Knoten und Überschreiten-Unfälle auf Geschäftsstraßen konnte gut eingegrenzt werden. Interessant ist das weitgehende Fehlen von Unfällen mit dem ruhenden Verkehr in der Mikroanalyse, wo nur die Unfälle des gegenläufigen Radverkehrs vertieft untersucht wurden. Die zeigt, dass die Radfahrer gegen die Richtung besser sichtbar sind als die Radfahrer, die sich in Fahrtrichtung der Einbahnstraße bewegen.

Diese Erkenntnisse entsprechen auch Ergebnissen aus früheren Untersuchungen, die in der Literaturanalyse ausgewertet wurden.

\subsection{Empfehlungen für geöffnete Einbahnstraßen}

In der Regel gestaltet sich die Öffnung von Einbahnstraßen für den Radverkehr unproblematisch, so dass auf besondere Maßnahmen verzichtet werden kann. Bei der Freigabe des Radfahrens in Gegenrichtung ist jedoch auf die Einhaltung der Vorgaben zur Öffnung von Einbahnstraßen gemäß VwV-StVO und den RASt 06 zu achten. Das betrifft vor allem die Beschilderung an den Knotenpunkten mit dem Zusatzzeichen „Radverkehr aus beiden Richtungen“. Falls sich in geöffneten Einbahnstraßen an Knotenpunkten Verkehrssicherheitsprobleme abzeichnen, sollte geprüft werden, ob die in den ERA oder RASt empfohlenen unterstützenden Markierungen und Beschilderungen erfolgen können:, wie z. B. die Anordnung von Gefahrenzeichen 102 „Kreuzung oder Einmündung mit Vorfahrt von Rechts“ mit dem Zusatzzeichen 1000-30 (,beide Richtungen“)oder 101 „Gefahrstelle“ bzw. 138 „Radfahrer kreuzen“, mit dem Zusatzzeichen 100032 („Radverkehr aus beiden Richtungen“), wie es in den ERA bzw. den RASt empfohlen wird.

In Geschäftsstraßen mit sehr hohem Fußgängeraufkommen ist auch den Fußgängern die Einbahnstraße zu verdeutlichen, da Fußgänger die aus der Gegenrichtung kommenden Radfahrer häufig nicht wahrnehmen:

- Markierungen in Form von Piktogrammen und Pfeilen

- Neuordnung des Parkens, um notwendige Sichtfelder freizuhalten.

In geöffneten Einbahnstraßen sollte bei der Unfalldatenaufnahme auch die Fahrtrichtung mit erfasst werden. 


\section{Quellenverzeichnis}

ADFC BRAUNSCHWEIG: Fahrradstraßen in Braunschweig (online) http://www.adfcbraunschweig.de/sicherheit-verkehr/verkehrspolitik/fahrradstrassen.html (29.02.16)

ALRUTZ, D.,/STELLMACHER-HEIN, J. (1997): Sicherheit des Radverkehrs auf Erschließungsstraßen, Berichte der Bundesanstalt für Straßenwesen, Heft V 37; Bergisch-Gladbach

BUNDESMINISTERIUM FÜR VERKEHR, BAU- UND STADTENTWICKLUNG - BMVBS (Hrsg. 2011): Hochrechnungsmodell von Stichprobenzählungen für den Radverkehr (FE 77.495/2008). Bearbeitet von der TU Dresden und PGV. Berlin/Dresden

BUNDESMINISTERIUM FÜR VERKEHR, BAU- UND STADTENTWICKLUNG - BMVBS (Hrsg. 2013): Nationaler Radverkehrskongress Münster, Berlin

BUNDESMINISTERIUM FÜR VERKEHR, BAU- UND STADTENTWICKLUNG - BMVBS (2015): Allgemeine Verwaltungsvorschrift Straßenverkehrs-Ordnung (VwVStVO), (online): http://www.verwaltungsvorschriften-iminternet.de/bsvwvbund_26012001_S3236420014.htm (29.02.16)

DELBRESSINE, R. (2013): The traffic safety of cycling roads in the Netherlands, Masterarbeit im Fach Civil Engineering an der Technischen Universität Delft.

FORSCHUNGSGESELLSCHAFT FÜR STRASSEN- UND VERKEHRSWESEN - FGSV (Hrsg. 2006): Richtlinie für die Anlage von Stadtstraßen. RASt 06 - Köln

FORSCHUNGSGESELLSCHAFT FÜR STRASSEN- UND VERKEHRSWESEN - FGSV (Hrsg. 2010): Empfehlungen für Radverkehrsanlagen. ERA - Köln

FORSCHUNGSGESELLSCHAFT FÜR STRASSEN- UND VERKEHRSWESEN - FGSV (Hrsg. 2015): Handbuch für die Bemessung von Straßenverkehrsanlagen. HBS - Köln

GESAMTVERBAND DER DEUTSCHEN VERSICHERUNGSWIRTSCHAFT - GDV (2013): Abbiegeunfälle Pkw/Lkw und Fahrrad. Forschungsbericht Nr. 21. Berlin.

GESAMTVERBAND DER DEUTSCHEN VERSICHERUNGSWIRTSCHAFT - GDV (2014): Geschwindigkeitsmessungen in Berlin (online): http://udv.de/de/medien/mitteilungen/geschwindigkeitsmoral-berlin-recht-gut (29.2.16)

GESAMTVERBAND DER DEUTSCHEN VERSICHERUNGSWIRTSCHAFT - GDV (2015): Geschwindigkeitsmessungen in Köln (online): http://udv.de/de/publikationen/praesentationen/geschwindigkeitsmessungen -koeln

PGV/BIS; Planungsgemeinschaft Verkehr, Hannover/ Büro für integrierte Stadt- und Verkehrsplanung, Bonn (2001): Verkehrssicherheit in Einbahnstraßen mit gegengerichtetem Radverkehr, Berichte der Bundesanstalt für Straßenwesen Heft V83; Bergisch Gladbach 
PS/PGV; Planerbüro Südstadt, Köln/ Planungsgemeinschaft Verkehr, Hannover (2000): Begleitforschung zu den Fahrradfreundlichen Städten und Gemeinden in NRW, Ministerium für Wirtschaft und Mittelstand, Energie und Verkehr des Landes Nordrhein-Westfalen (Hrsg.), Düsseldorf 2000

RETTENBACHER, P.( 2000): Radunfälle und Radfahren gegen die Einbahn. In:

Radverkehr: Fördern und sichern. 'Tagungsband zum Fahrradkongress am 11.10.2000 in Salzburg, 0. O.

STADT BONN (Hrsg. 2012): Fahrradstraßenkonzept der Stadt, Bonn,

STADT BRAUNSCHWEIG (o.J.): Fahrradstraßen in Braunschweig (online) https://www.braunschweig.de/leben/stadtplan_verkehr/radverkehr/fahrradstr assen.html Infoflyer zur Einrichtung von Fahrradstraßen, (29.02.16)

STADT BREMEN (2015): Bremer Leitlinien für die Gestaltung von Fahrradstraßen, Bremen

STADT HERFORD (Hrsg. 1997): Begleituntersuchung fahrradfreundliche Stadt Herford, Hannover

STADT MÜNSTER (Hrsg. 1993): -Fahrradstraße Schillerstraße, Münster

STADT MÜNSTER (Hrsg. 1995): Radfahrer in unechten Einbahnstraßen, Münster

STATISTISCHES BUNDESAMT (2014): Verkehr - Verkehrsunfälle. In: Fachserie 8, Reihe 7, Wiesbaden 


\section{Anhang}

Anhang 1: Fragebogen der Kommunalbefragung, Phase 1

Anhang 2: $\quad$ Fragebogen der Kommunalbefragung, Phase II Fahrradstraßen

Anhang 3: Fragebogen der Kommunalbefragung, Phase II geöffnete Einbahnstraßen

Anhang 4: Fragebogen der Verkehrsteilnehmerbefragung. 
Anhang 1

\section{Fragen zur Phase I der Kommunalbefragung}

im Projekt

Sicherheitsbewertung von Fahrradstraßen und der Öffnung von Einbahnstraßen 
Frage 1: Bitte vervollständigen Sie die unten stehenden Angaben.

- Anrede

- Vorname

- Name

- Funktion

- Firma

- Straße/Nr.

- Postleitzahl/Ort

- E-Mail

- Telefonnr.

Frage 2: Bitte vervollständigen Sie die folgenden Angaben:

- Name der Stadt/Gemeinde

- Einwohnerzahl

Frage 3: Wurde für Ihre Kommune der Modal Split erhoben?

- Ja, im Jahr:

- Ja, auf Kreisebene etc. im Jahr:

- Nein

- Weiß nicht

Frage 4: Bitte nennen Sie uns die Anteile der einzelnen Verkehrsarten im Modal Split:

- Anteil Kfz:

- Anteil Rad:

- Anteil zu Fuß:

- Anteil ÖV:

Frage 5: Gibt es in Ihrer Kommune ein Radverkehrskonzept als einzelnes Konzept oder auch im Rahmen der übergeordneten Verkehrsplanung, z.B. im Verkehrsentwicklungsplan (VEP)?

- Ja

- Nein

- Weiß nicht

Frage 6: Wann wurde das Radverkehrskonzept erstellt?

HINWEIS: Bitte tragen Sie die Jahreszahl z.B. wie folgt ein: 2003

- (Jahreszahl)

- Weiß nicht

Frage 7: Ist ein Radverkehrskonzept für die nächste Zeit geplant?

- Ja, im Jahr:

- Nein

- Weiß nicht 
Frage 8: Sofern Ihnen bekannt, nennen Sie uns bitte die für Ihre Kommune für die Unfalldatenerfassung und -bearbeitung zuständige Polizeidienststelle.

- Vorname

- Name

- Firma

- Straße/Nr.

- Postleitzahl/Ort

- E-Mail

- Telefonnr.

Hinweis: Bitte machen Sie nun Angaben zu den Fahrradstraßen in Ihrer Kommune.

Frage 9: Gibt es Fahrradstraßen in Ihrer Kommune?

- Ja

- Nein

- Weiß nicht

Frage 10: Beabsichtigen Sie zukünftig (weitere) Fahrradstraßen umzusetzen?

- Ja

- Vielleicht

- Nein

- Weiß nicht

Frage 11: Welche Gründe sprechen gegen einen Einsatz von Fahrradstraßen?

- Politischer Grundsatzbeschluss

- Bisher noch kein passender Einsatzzweck

- Aus Gründen der Verkehrssicherheit

- Sonstiges, und zwar:

- Weiß nicht

Frage 12: Wie wird die Einrichtung von Fahrradstraßen durch Öffentlichkeitsarbeit begleitet?

- Wir haben eine allgemeine Information für alle Fahrradstraßen heraus gegeben (z.B. Faltblatt)

- Wir geben für jede Fahrradstraße oder jeden Stadtteil/Stadtbezirk eine straßenspezifische Information heraus, z.B. ein Faltblatt

- Wir geben zu jeder neu eingerichteten Fahrradstraße eine Presseinformation heraus

- Gar nicht

- Sonstiges: Weiß nicht

Frage 13: Wird oder wurde die Einrichtung von Fahrradstraßen durch eine Vorher-/NachherUntersuchung o.ä. begleitet?

- Ja, generell

- Ja, aber nur in Einzelfällen

- Nein

- Weiß nicht 
Frage 14: Wurde in Ihrer Kommune die Einrichtung einer Fahrradstraße schon einmal wieder rückgängig gemacht?

- Ja, weil:

- Nein

- Weiß nicht

Frage 15: Auf welcher Grundlage richten Sie Fahrradstraßen ein?

Mehrfachantwort möglich

- Es gibt einen politischen Beschluss, Fahrradstraßen einzurichten

- Es gibt ein Fahrradstraßenkonzept für die Kommune

- Jede Fahrradstraße wird einzeln politisch beschlossen

- Fahrradstraßen können auch ohne politischen Beschluss eingerichtet werden

- Sonstiges, und zwar:

- Weiß nicht

Frage 16: Welche Bedingungen müssen erfüllt sein, damit eine Straße in eine Fahrradstraße umgewandelt wird?

Mehrfachantwort möglich

- Liegt im Verlauf einer Schulwegeroute

- Liegt im Hauptradroutennetz

- Zur Schließung von Netzlücken im Radverkehrsnetz

- Liegt im Verlauf einer touristischen Radroute / Themenroute

- Hohe Radverkehrsstärke auf der Strecke

- Radverkehr ist die dominierende Verkehrsart auf dieser Strecke

- Sonstiges, und zwar:

- Weiß nicht

Frage 17: Gibt es Gestaltungsprinzipien für Fahrradstraßen in Ihrer Kommune?

Mehrfachantwort möglich

- Ja

- Ja, Upload einer Datei

- Nein

- Weiss nicht

Frage 18: Wie viele Fahrradstraßen gibt es in Ihrer Kommune?

- Anzahl Fahrradstraßen gesamt:

- Geschätzte Gesamtlänge (in km):

- Anzahl Fahrradstraßen innerorts:

- Geschätzte Gesamtlänge (in km):

- Anzahl Fahrradstraßen außerorts:

- Geschätzte Gesamtlänge (in km):

Frage 19: Folgende Fahrradstraßen sind in unserer Kommune eingerichtet:

- Straße 1

Hinweis: Bitte machen Sie nun Angaben zu den für den Radverkehr geöffneten Einbahnstraßen in Ihrer Kommune. 
Frage 20: Wie viele Einbahnstraßen gibt es in Ihrer Kommune?

- Keine

- Weniger als 10

- $10-20$

- $21-50$

- 51-100

- Mehr als 100

- Weiß nicht

Frage 21: Haben Sie Einbahnstraßen für den Radverkehr in Gegenrichtung geöffnet?

- Ja

- Nein, keine

- Weiß nicht

Frage 22: Wie viele Einbahnstraßen sind für den Radverkehr in Gegenrichtung geöffnet?

- (Anzahl)

- Weiß nicht

Frage 23: Gibt es die Absicht, zukünftig Einbahnstraßen für den Radverkehr in Gegenrichtung zu öffnen?

- Ja

- Nein

- Weiß nicht

Frage 24: Wie viele Einbahnstraßen sollen für den Radverkehr in Gegenrichtung geöffnet werden?

- (Anzahl)

- Weiß nicht

Frage 25: Wie wird die Öffnung von Einbahnstraßen für den Radverkehr in Gegenrichtung durch Öffentlichkeitsarbeit begleitet?

Mehrfachantwort möglich

- Wir haben eine allgemeine Information für alle geöffneten Einbahnstraßen heraus gegeben (z.B. Faltblatt)

- Wir geben für jede Einbahnstraße oder jeden Stadtteil/Stadtbezirk eine straßenspezifische Information heraus, z.B. ein Faltblatt

- Wir geben zu jeder neu geöffneten Einbahnstraße eine Presseinformation heraus

- Sonstiges:

- Gar nicht

- Weiß nicht

Frage 26: Wird oder wurde die Öffnung von Einbahnstraßen für den Radverkehr in Gegenrichtung durch Vorher-/Nachher-Untersuchungen o.ä. begleitet?

- Ja, generell

- Ja, aber nur in Einzelfällen

- Nein

- Weiß nicht

Frage 27: Wurde in Ihrer Kommune die Öffnung einer Einbahnstraße für den Radverkehr in Gegenrichtung schon einmal wieder rückgängig gemacht?

- Ja, weil: 
- Nein

- Weiß nicht

Frage 28: Hat sich in der Praxis bei der Öffnung von Einbahnstraßen durch die letzten Neuregelungen in der StVO etwas geändert?

- Ja, und zwar wie folgt:

- Nein

- Weiß nicht

Frage 29: Folgende Einbahnstraßen sind in unserer Kommune geöffnet:

- $\quad$ Straße 1

Frage 30: Haben Sie noch Anmerkungen für uns?

- $\quad($ Text) 
Anhang 2

Fragen zur Phase II der Kommunalbefragung,

Teil Fahrradstraßen

im Projekt

Sicherheitsbewertung von Fahrradstraßen und der Öffnung von Einbahnstraßen 
Name der Fahrradstraße

Frage 1: Bitte geben Sie die Länge in km der besagten Straße an.

- (Länge in $\mathrm{km}$ )

- Weiß nicht

Frage 2: Wann wurde die Fahrradstraße eingerichtet?

- (Monat)

- (Jahr)

- Weiß nicht

Frage 3: Ist diese Fahrradstraße eine Einbahnstraße für den Kfz-Verkehr?

- Ja

- Nein

- Weiß nicht

Frage 4: Welche zusätzlichen Verkehrsarten bzw. Nutzer sind auf dieser Fahrradstraße zugelassen?

Mehrfachantwort möglich

- Kfz frei

- Anlieger frei

- Sonstiges:

- Keine

- Weiß nicht

Frage 5: Ist die Zulassung weiterer Verkehrsarten auf bestimmte Uhrzeiten beschränkt?

- Ja, und zwar:

- Nein

- Weiß nicht

Frage 6: Welche Maßnahmen wurden bei der Einrichtung dieser Fahrradstraße eingesetzt? Mehrfachantwort möglich

- Beschilderung

- Bauliche Einengungen an Einmündungen oder Kreuzungen

- Aufpflasterungen in Knotenpunktbereichen

- Begrenzung der Fahrbahnbreite durch Markierungen

- Markierung von Fahrrad-Piktogrammen auf der Fahrbahn

- Markierung von Zeichen 237 „Radweg“ auf der Fahrbahn

- Markierung von Zeichen 244 „Fahrradstraße“ auf der Fahrbahn

- Sonstiges:

- Weiß nicht 
Frage 7: Wie wird das Autoparken in dieser Fahrradstraße geregelt?

Mehrfachantwort möglich

- Einseitig Parken auf der Fahrbahn erlaubt

- Beidseitig Parken auf der Fahrbahn erlaubt

- Wechselseitiges Parken auf der Fahrbahn erlaubt

- Einseitig Parken in den Nebenanlagen

- Beidseitig Parken in den Nebenanlagen

- Anders, und zwar:

- Weiß nicht

Frage 8: Gibt es im Verlauf dieser Fahrradstraße Querungsstellen oder Querungshilfen für Fußgänger?

Mehrfachantwort möglich

- Ja

- Ja, signalisiert

- Ja, als Fußgängerüberweg (Zebrastreifen)

- Ja, als Fahrbahneinengung / Mittelinsel

- Ja, sonstiges:

- Nein

- Weiß nicht

Frage 9: Vorfahrtsituation an Einmündungen / Knotenpunkten

Diese Fahrradstraße ist ...

- ...an allen Einmündungen und Knotenpunkten bevorrechtigt, außer an kreuzenden Hauptverkehrsstraßen

- ...mit rechts vor links-Regelung an allen Knotenpunkten geregelt

- ...nicht einheitlich Vorfahrt geregelt

- Sonstiges, und zwar:

- Weiß nicht

Frage 10: Verkehrsbelastung im Querschnitt der Fahrradstraße (beide Richtungen gemeinsam) Bitte vervollständigen Sie die folgenden Angaben:

- Kfz-Belastung (Fahrzeuge/Tag)

- Radverkehrs-Belastung (Radfahrer/Tag)

- Buslinienverkehr

- Lkw-Verkehr (über 5\%)

- Weiß nicht

Frage 11: Welche Netzbedeutung hat die Fahrradstraße für den Radverkehr? Mehrfachantwort möglich

- Bestandteil der Hauptrouten des Radverkehrsnetzes

- Bestandteil der Nebenrouten des Radverkehrsnetzes

- Bestandteil eines Radschnellwegs

- Bestandteil einer touristischen Themenroute / Radroute

- Bestandteil einer Schulwegeroute

- Sonstiges:

- Weiß nicht

Frage 12: Welche Funktion hat die Fahrradstraße?

Mehrfachantwort möglich 
- Verbindungsfunktion für den Radverkehr

- Sammelfunktion für den Radverkehr

- Erschließung von wichtigen Quellen/Zielen des Alltagsverkehrs (z.B. Schule, Arbeitsplatzschwerpunkt etc.)

- Erschließung von wichtigen Quellen/Zielen des Tourismus

- Sicherstellung einer attraktiven Kfz-freien /oder -armen Radverkehrsverbindung

- Sonstiges:

- Weiß nicht

Frage 13: Wie wird diese Fahrradstraße von Ihnen im Hinblick auf verkehrssicherheitsrelevante Konflikte bewertet?

- Konflikte, aber keine Unfälle

- Unfallträchtig

- Unproblematisch

- Weiß nicht

Frage 14: Wurde die Einrichtung dieser Fahrradstraße durch eine Vorher-/Nachher-Untersuchung begleitet?

Bitte stellen Sie uns diese Untersuchung, wenn möglich, zur Verfügung.

- Ja

- Ja, Upload Untersuchung

- Nein

- Weiß nicht

Frage 15: Sie haben angegeben, dass die Einrichtung dieser Fahrradstraße durch eine Vorher/Nachher-Untersuchung begleitet wurde. Was wurde untersucht?

Mehrfachantwort möglich

- Unfälle

- Verhalten der Verkehrsteilnehmer (Beobachtung)

- Anzahl Verkehrsteilnehmer (Zählung)

- Sonstiges:

- Weiß nicht

Frage 16: Wie wurde die Einrichtung dieser Fahrradstraße durch Öffentlichkeitsarbeit begleitet? Mehrfachantwort möglich

- Ja, Allgemeine Information, z.B. Faltblatt

- Ja, Stadtteil- oder straßenspezifische Informationen, z.B. Faltblatt

- Ja, Presseinformation

- Ja, Sonstiges:

- Nein

- Gar nicht

- Weiß nicht 
Frage 17: Liegen Anregungen oder Beschwerden aus der Bürgerschaft für diese Einbahnstraße vor?

- Ja, folgende:

- Nein

- Weiß nicht

Frage 18: Bitte stellen Sie uns, sofern vorhanden, eine Entwurfskizze oder ähnliches der Fahrradstraße zur Verfügung.

- Ja, Upload

- Kein Lageplan vorhanden

Frage 19: Bitte stellen Sie uns eventuell vorhandene Fotos der Fahrradstraße zur Verfügung.

- Ja, Upload

- Keine Fotos vorhanden

Frage 20: Haben Sie noch Anmerkungen für uns?

- $($ Text $)$ 
Anhang 3

Fragen zur Phase II der Kommunalbefragung,

Teil Geöffnete Einbahnstraßen

im Projekt

Sicherheitsbewertung von Fahrradstraßen und der Öffnung von Einbahnstraßen 
Für jede geöffnete Einbahnstraße ist ein eigener Fragebogen auszufüllen.

(Name der Straße)

Frage 1: Bitte geben Sie die Länge in $\mathrm{km}$ der besagten Straße an.

- $\mathrm{km}$

- Weiß nicht

Frage 1: Wie hoch ist dort die erlaubte Höchstgeschwindigkeit?

- maximal $50 \mathrm{~km} / \mathrm{h}$

- maximal $30 \mathrm{~km} / \mathrm{h}$

- maximal $20 \mathrm{~km} / \mathrm{h}$

- maximal $10 \mathrm{~km} / \mathrm{h}$

- Schritttempo

- Anderes Tempo, und zwar:

- Weiß nicht

Frage 2: Gibt es besondere Verkehrsregelungen auf / in dieser Straße? Mehrfachantwort möglich

- T-30-Zone

- verkehrsberuhigter Bereich

- Parkverbotszone

- Fahrradstraße

- Umwandlung in unechte Einbahnstraße (einseitiges Einfahrverbot, die Straße darf aber in beiden Richtungen befahren werden)

- Sonstiges:

- Keine

- Weiß nicht

Frage 3: Seit wann ist die Straße für den Radverkehr geöffnet?

- (Monat)

- (Jahr)

- Weiß nicht

Frage 4: Welche Maßnahmen wurden eingesetzt, um die Einbahnstraße für den Radverkehr zu öffnen?

Mehrfachantwort möglich

- Beschilderung

- Markierung

- Bauliche Umgestaltung

- Neuordnung des Parkens

- Sonstiges:

- Weiß nicht 
Frage 5: Wie wird der Radverkehr in Gegenrichtung in dieser Einbahnstraße überwiegend geführt?

- Fahrbahn im Mischverkehr

- Fahrbahn auf Schutzstreifen

- Fahrbahn auf Radfahrstreifen

- Nebenanlagen als benutzungspflichtiger Radweg

- Nebenanlagen als nicht benutzungspflichtiger Radweg

- Nebenanlagen als Gehweg, Radfahrer frei

- Sonstiges

- Weiß nicht

Frage 6: Wurde die Öffnung dieser Einbahnstraße für den Radverkehr in Gegenrichtung durch Öffentlichkeitsarbeit begleitet?

Mehrfachantwort möglich

- Ja, Allgemeine Information, z.B. Faltblatt

- Ja, Stadtteil- oder straßenspezifische Informationen, z.B. Faltblatt

- Ja, Presseinformation

- Ja, Sonstiges:

- Nein

- Weiß nicht

Frage 7: Wurde die Öffnung dieser Einbahnstraße für den Radverkehr in Gegenrichtung durch eine Vorher-/Nachher-Untersuchung begleitet?

Bitte stellen Sie uns diese Untersuchung, wenn möglich, zur Verfügung.

- Ja

- Ja, Upload

- Nein

- Weiß nicht

Frage 8: Sie haben angegeben, dass die Öffnung dieser Einbahnstraße für den Radverkehr in Gegenrichtung durch eine Vorher-/Nachher-Untersuchung begleitet wurde. Was wurde untersucht?

Mehrfachantwort möglich

- Unfälle

- Verhalten der Verkehrsteilnehmer (Beobachtung)

- Anzahl Verkehrsteilnehmer (Zählung)

- Sonstiges:

- Weiß nicht

Frage 9: Liegen Anregungen oder Beschwerden aus der Bürgerschaft für diese Einbahnstraße vor?

- Ja, folgende: (Text)

- Nein

- Weiß nicht

Frage 10: Bitte stellen Sie uns, sofern vorhanden, eine Entwurfskizze oder ähnliches der Einbahnstraße zur Verfügung.

- Ja, Upload

- Kein Lageplan vorhanden

Frage 11: Bitte stellen Sie uns eventuell vorhandene Fotos der Einbahnstraße zur Verfügung. 
- Ja, Upload

- Keine Fotos vorhanden

Frage 12: Haben Sie noch Anmerkungen für uns?

(Text) 
Anhang 4

\section{Fragen zur Verkehrsteilnehmerbefragung}

im Projekt

Sicherheitsbewertung von Fahrradstraßen und der Öffnung von Einbahnstraßen 
Anweisung für den Interviewer:

Allgemein: Wir benötigen unbeeinflusste Antworten. Wenn z.B. eine Gruppe Schüler kommt, dann bitte einzeln beiseite nehmen und befragen. Die Gruppe soll keine Kommentare dazu geben und auch die Antworten nicht mit bekommen.

Erst ab einem Alter von 14 Jahren befragen, bei Jüngeren ist das Einverständnis der Eltern erforderlich, das uns aber nicht vorliegt.

Zu Frage 5: Antwortmöglichkeiten nicht vorlesen, sondern als offene Frage stellen und die Antworten in den Vorgaben entsprechend eintragen. Wenn keine passende Vorgabe vorhanden ist, die Leerzeilen nutzen und Stichworte notieren.

Wenn die Vorgaben in den freien Antworten enthalten sind, werden die korrespondierenden Fragen nicht gestellt.

Zu Frage 12: Bitte nur die ersten beiden Antwortmöglichkeiten vorlesen.

Einleitung durch den Interviewer:

Guten Tag. Wir führen eine Befragung von Verkehrsteilnehmern zur Verkehrssicherheit bei Radfahrern und Autofahrern durch. Darf ich Ihnen dazu einige Fragen stellen? Es wird nur wenige Minuten dauern. 
Fragebogen- $\mathrm{Nr}$.

Frage 1: Welches Verkehrsmittel nutzen Sie am häufigsten?

() Kfz () Rad () Bus/Bahn () gehe zu Fuß () nutze alle VM fast gleich häufig () weiß nicht ( ) k.A. Frage 2: Wie oft nutzen Sie das Fahrrad?

() Täglich () Mehrmals wöchentlich () Seltener () weiß nicht () k.A.

Frage 3: Wie oft sind Sie in dieser Straße hier?

() Täglich () Mehrmals wöchentlich () Seltener () weiß nicht () k.A.

Frage 4: In welcher Art Straße nach StVo befinden wir uns hier?

(Nicht vorlesen, sondern Antwort abwarten!)

() SpielstraßeZ.325 () Einbahnstraße () Fahrradstraße () Tempo 30-Zone ()Wohnstraße

() Hauptverkehrsstraße () Kombination Einbahnstraße/Fahrradstraße () weiß nicht

Bitte nennen: Wir sind hier in einer Fahrradstraße und die Grafik des Verkehrsschildes zeigen

Frage 5: Bitte nennen Sie spezielle Regelungen, die allgemein in Fahrradstraßen gelten:

An twortmöglichkeiten nicht vorlesen, sondern als offene Frage stellen und die Antworten in den Vorgaben entsprechend eintragen. Wenn keine passende Vorgabe vorhanden ist, die Leerzeilen nutzen und Stichworte notieren.

Wenn die Vorgaben in den freien Antworten enthalten sind, werden die korrespondierenden Fragen nicht gestellt.

() kann keine nennen $\rightarrow$ direkt weiter zu Frage 6 () weiß nicht () folgende:

() 5.1: Freie Antwort (bei Kreuz, $\rightarrow$ Frage 6 fällt aus)

\begin{tabular}{|l|l|}
\hline ( ) Frage 6: Welche Fahrzeuge dürfen in Fahrradstraßen fahren? \\
\hline $\begin{array}{ll}\text { ( ) Alle, es gibt keine Einschränkungen } & \text { ( ) Eigentlich nur Fahrräder, andere Fahrzeuge } \\
\text { () Alle ausser Lkw, } & \text { können aber erlaubt werden } \\
\text { () Nur Fahrräder ohne Ausnahme } & \text { ( ) weiß nicht }\end{array}$ \\
\hline
\end{tabular}

( ) 5.2: Freie Antwort (bei Kreuz $\rightarrow$ Frage 7 fällt aus)

\begin{tabular}{|l|l|}
\hline $\begin{array}{l}\text { ( ) Frage 7: Durfen Radfahrer in Fahrrad- } \\
\text { straßen auch nebeneinander fahren? }\end{array}$ & ( ) Ja ( ) nein ( ) weiß nicht \\
\hline
\end{tabular}

() 5.3: Freie Antwort (bei Kreuz $\rightarrow$ Frage 8 fällt aus)

\begin{tabular}{|l|l|}
\hline $\begin{array}{l}\text { ( ) Frage 8: Haben Radfahrer in } \\
\text { Fahrradstraßen an Kreuzungen und } \\
\text { Einmundungen immer Vorfahrt? }\end{array}$ & ( ) Ja ( ) nein ( ) weiß nicht \\
\hline
\end{tabular}

() 5.4: Freie Antwort (bei Kreuz $\rightarrow$ Frage 9 fällt aus)

\begin{tabular}{|l|l|}
\hline $\begin{array}{l}\text { Frage 9: Durfen Autos die Radfahrer } \\
\text { uberholen? }\end{array}$ & ( ) la ( ) nein () weiß nicht \\
\hline
\end{tabular}

( ) $5: 5$ Freie Antwort(bei Kreuz $\rightarrow$ Frage10 fällt aus)

\begin{tabular}{|c|c|}
\hline $\begin{array}{l}\text { () Frage 10: Wie schnell darf man in } \\
\text { Fahrradstraßen max. fahren? }\end{array}$ & $\begin{array}{l}\text { () } 10 \text { () } 20 \text { () } 30 \text { () } 50 \mathrm{~km} / \mathrm{h} \quad \text { () weiß } \\
\text { nicht }\end{array}$ \\
\hline
\end{tabular}

( ) 5:6 Freie Antwort (bei Kreuz $\rightarrow$ Frage11 fältt aus)

\begin{tabular}{|l|l|}
\hline $\begin{array}{l}\text { ( ) Frage 11: Durfen Radler in der Mitte der } \\
\text { Fahrbahn fahren? }\end{array}$ & ( ) Ja ( ) nein () weiß nicht \\
\hline
\end{tabular}


Frage 12: thre persöntiche Einschatzung zur Verkehrssicherheit:

Interviewer: Bitte nur die ersten beiden Antwortmöglichkeiten vorlesen.

12.1 Machen die hier parkenden Autos Ihre Fahrt durch die Fahrradstraße eher () sicher oder () unsicher oder () neutral-wedernoch () weißs nicht () k.A.

12.2 Machen die hier querenden Fußgänger lhre Fahrt durch die Fahrradstraße eher () sicher oder () unsicher oder () neutral-weder noch () weiß nicht () k.A.

12.3 Macht die Gestaltung der Straße, also Markierungen, Schilder etc Ihre Fahrt duch die Fahrradstraße () sicher oder () unsicher oder () neutral-weder noch () weiß nicht () k.A.

12.4 Macht die Gestaltung von Anfang und Ende der Fahrradstraße, also Schilder, Markierungen etc Ihre Fahrt durch die Fahrradstraße eher

() sicheroder () unsicher oder () neutral-weder noch () weiß nicht () k.A.

12.5 Macht die Geschwindigkeit der hier fahrenden Autos thre Fahrt duch die Fahrradstraße eher () sicheroder () unsicher oder () neutral-wedernoch ( ) weiß nicht () kA.

12.6 Macht die Vorfahrtegelung in dieser Fahrradstraße Ihre Fahrt duch die Fahrradstraße eher () sicheroder () unsicher oder () neutral-wedernoch () weiß nicht () k.A.

12.7 Wie ist lhr Gesamteindruck dieser Fahrradstraße? Fühlen Sie sich bei lhrer Fahrt durch diese Straße eher
() sicher oder ( ) unsicher
oder ( ) neutral-weder noch
() weifs nicht
( ) $\mathrm{kA}$.

Frage 13: Kennen Sle noch weitere Fahrradstraßen in Ihrer Stadt?

() Ja () nein () weiß nicht

Frage 14: Meinen Sie, es solite noch mehr Fahrradstraßen in Ihrer Stadt geben?

() Ja () nein () weiß nicht

Um Ihre Antworten mit den anderen besser vergleichen zu können, benötigen wir noch:

Frage 15: Ihr Alter: Jahre () keine Angabe

() unter 16 Jahre () 16 - unter 18 () $18-29$ () $30-39$ () $40-49$ () $50-59$ () $60-69$ () $70+$ Vielen Dank für Ihre Mitarbeit!

Interviewer füllt aus

Geschlecht: () m () w () keine Angabe

Befragter nutzt: () Rad () Geht zu Fuß () Auto (Geht zu oder kommt von seinem Fahrzeug)

Standort: Stadt: Straße

Tag der Befragung

Uhrzeit

Interviewer 
Gesamtverband der Deutschen Versicherungswirtschaft e. V.

Wilhelmstraße 43 / 43G, 10117 Berlin

Postfach 0802 64, 10002 Berlin

Telefon 030 / 2020 - 50 00, Fax 030 / 2020 - 6000

Internet: www.gdv.de, www.udv.de 